\title{
Assembly of Primary (Hetero)Arylamines via CuI/Oxalic Diamides-Catalyzed Coupling of Aryl Chlorides and
}

\section{Ammonia}

Mengyang Fan, Wei Zhou, Yongwen Jiang and Dawei Ma*

State Key Laboratory of Bioorganic and Natural Products Chemistry, Shanghai Institute of Organic Chemistry, Chinese Academy of Science, 345 Lingling Lu, Shanghai 200032, China

E-mail: $\underline{\text { madw@mail.sioc.ac.cn }}$

\section{Supporting Information}

Table of contents

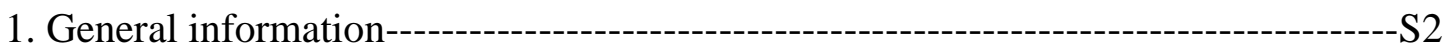

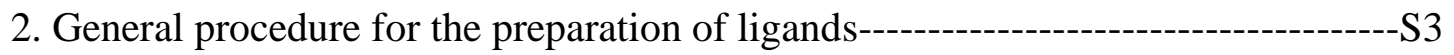

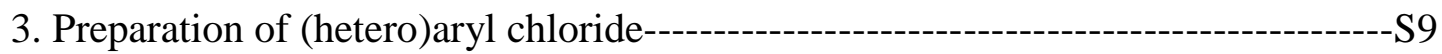

4. General procedure for the CuI-catalyzed monoarylation of ammonia--------------S10

5. Copies of ${ }^{1} \mathrm{H}$ and ${ }^{13} \mathrm{C}$ spectrum of ligands and (hetero)aryl amines-----------------S27 


\section{General Information}

Reagents: All commercial materials were used as received unless otherwise noted. THF was distilled from $\mathrm{Na}$. CuI $(98 \%, \mathrm{~J} \& \mathrm{~K})$ was washed with refluxing THF in Soxhlet extractor for $12 \mathrm{~h}$ before used. Ammonia aqueous solution (AR, 25-28\%, Suzhou JIMCEL H\&N Electronic Material Co.), $\mathrm{K}_{3} \mathrm{PO}_{4}$ (reagent grade, $\geq 98 \%$, Sigma Aldrich) and DMSO (anhydrous, $\geq 99.9 \%$, Sigma Aldrich) were used in the CuIcatalyzed reactions. Flash chromatography was performed using 230-400 mesh SiliaFlash ${ }^{\circledR}$ P60 (Silicycle Inc.).

Reactions: All reactions for the CuI-catalyzed arylation of ammonia were set up on bench-top in the open air and carried out in re-sealable test tubes with Teflon septa under an argon atmosphere. Unless otherwise noted, the reaction test tubes were cooled to room temperature prior to other operations. Unless otherwise noted, the solvents and the solutions of reagents/reactants were transferred via microsyringe or plastic syringe (fitted with metal needle) into the reaction test tubes under a positive argon pressure.

Instruments: NMR spectra were recorded on Bruker Ultrashield ${ }^{\mathrm{TM}} 400$ Plus, Agilent Technologies 400/54 Premium Shielded, Agilent Technologies 500/54 Premium Shielded instruments and calibrated using residual solvent peaks as internal reference. Multiplicities are recorded as: $\mathrm{br}=$ broad, $\mathrm{s}=$ singlet, $\mathrm{d}=$ doublet, $\mathrm{t}=$ triplet, $\mathrm{q}=$ quartet, hept $=$ heptet, $\mathrm{dd}=$ doublet of doublets, $\mathrm{m}=$ multiplet. High resolution mass experiments were operated on a Bruker Daltonics, Inc. APEXIII 7.0 TESLA FTMS instrument and a Thermo Fisher Scientific LTQ FT Ultra instrument. 


\section{General Procedures for the Preparation of Ligands}<smiles>[R]Oc1cccc(NC(=O)C(=O)OC)c1</smiles>

General Procedure A: To a solution of the corresponding aniline (1.0 eq.) in THF (0.2 M) was added $\mathrm{Et}_{3} \mathrm{~N}$ (1.2 eq.). Mono-methyl oxalyl chloride (1.1 eq.) was then added to the solution slowly under ice-water bath. After the resulting mixture was stirred at room temperature for $1 \sim 2 \mathrm{~h}$, it was filtered and the white solid $\left(\mathrm{Et}_{3} \mathrm{~N} \cdot \mathrm{HCl}\right)$ on filter paper was washed with THF (other solvents such as EtOAc and DCM should be avoided because $\mathrm{Et}_{3} \mathrm{~N} \cdot \mathrm{HCl}$ would dissolve in them). The filtrate was concentrated in vacuo and the resulting residue was purified by silica gel flash chromatography to afford the corresponding methyl $\mathrm{N}$-aryloxamate. (Note: Monomethyl oxalyl chloride can be replaced by mono-ethyl oxalyl chloride to afford the corresponding ethyl $N$-aryloxamate.)

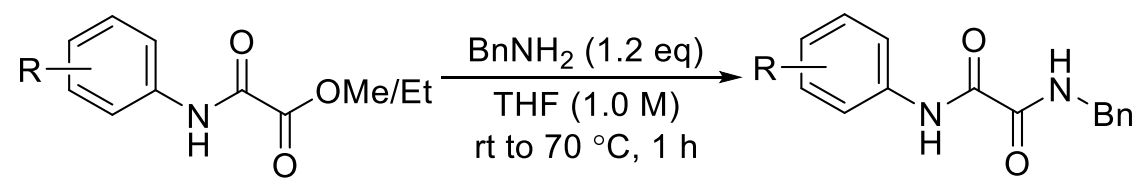

General Procedure B: To a solution of the corresponding methyl $N$-aryloxamate or ethyl $\mathrm{N}$-aryloxamate (1.0 eq.) in THF (1.0 M) was added $\mathrm{BnNH}_{2}(1.2$ eq.) at room temperature. After the resulting mixture was stirred under $70{ }^{\circ} \mathrm{C}$ for $1 \mathrm{~h}$, it was cooled to room temperature in the air and then to $-18{ }^{\circ} \mathrm{C}$ in refrigerator. In most cases, the products would precipitate out as crystals. If no solid precipitated, hexane was added to the mixture until the products precipitated out. The mixture was filtered and the 
precipitates were collected and washed with small amount of cold diethyl ether to afford the corresponding $N$-benzyl- $N$ '-aryloxalamide. They were pure enough to be used without further purification. (Note: $\mathrm{BnNH}_{2}$ can be replaced by other aliphatic amines to afford the corresponding $N$-alkyl- $N$ '-aryloxalamide.)

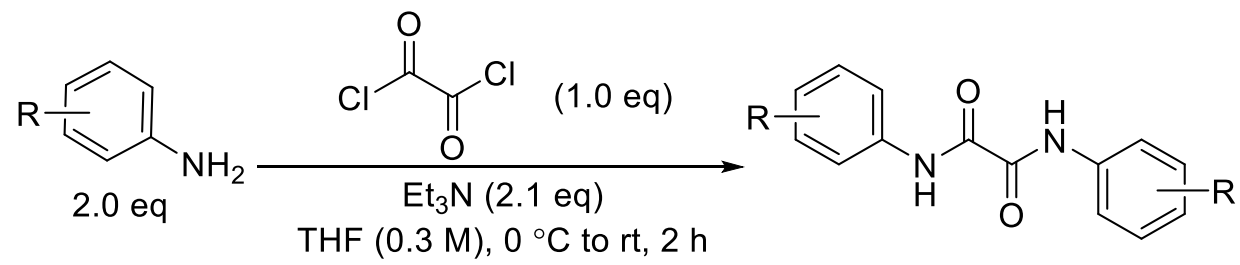

General Procedure C: To a solution of the corresponding aniline (2.0 eq.) in THF $(0.3 \mathrm{M})$ was added $\mathrm{Et}_{3} \mathrm{~N}$ (2.1 eq.). Oxalyl chloride (1.0 eq.) was then added to the solution slowly under ice-water bath. After the resulting mixture was stirred at room temperature for $2 \mathrm{~h}$, it was concentrated in vacuo to remove the solvent and water was added to the resulting residue to dissolve $\mathrm{Et}_{3} \mathrm{~N} \cdot \mathrm{HCl}$. Then the slurry was filtered and the precipitates on filter paper were washed with water and cold diethyl ether. These precipitates were dried in vacuo to afford the corresponding $N, N^{\prime}$-bisaryloxalamide. They were pure enough to be used without further purification. (Note: substituted aniline can be replaced by other aliphatic amines to afford the corresponding $N, N^{\prime}$ bisalkyloxalamide.)<smiles>[Y]C(=O)Nc1c(OC)cc(OC)cc1OCC(=O)Nc1c(OC)cc(OC)cc1OC</smiles>

The $\mathbf{L} 1^{1}$ was prepared from 2,4,6-trimethoxyaniline and oxalyl chloride following the general procedure $\mathrm{C}$ as a pale grey powder in $93 \%$ yield. ${ }^{1} \mathrm{H}$ NMR $(400 \mathrm{MHz}, d 6-$ 
DMSO) $\delta 9.33(\mathrm{~s}, 2 \mathrm{H}), 6.29(\mathrm{~s}, 4 \mathrm{H}), 3.80(\mathrm{~s}, 6 \mathrm{H}), 3.75(\mathrm{~s}, 12 \mathrm{H}) ;{ }^{13} \mathrm{C}$ NMR $(100 \mathrm{MHz}$, $\left.\mathrm{CDCl}_{3}\right) \delta 160.4,158.6,156.2,106.2,91.1,56.1,55.7$.<smiles>CC(=O)Nc1c(C)cc(C)cc1C</smiles>

L2

The $\mathbf{L 2}^{2}$ was prepared from 2,4,6-trimethylaniline and oxalyl chloride following the general procedure $\mathrm{C}$ as a white solid in $86 \%$ yield. ${ }^{1} \mathrm{H}$ NMR $\left(400 \mathrm{MHz}, \mathrm{CDCl}_{3}\right) \delta 8.81$ $(\mathrm{s}, 2 \mathrm{H}), 6.94(\mathrm{~s}, 4 \mathrm{H}), 2.30(\mathrm{~s}, 6 \mathrm{H}), 2.24(\mathrm{~s}, 12 \mathrm{H}) ;{ }^{13} \mathrm{C} \mathrm{NMR}\left(100 \mathrm{MHz}, \mathrm{CDCl}_{3}\right) \delta$ $158.4,137.7,134.8,129.8,129.2,21.1,18.5$.<smiles>COc1cc(C)c(NC(C)=O)c(C)c1</smiles>

The L3' was prepared from 3,5-dimethyl-4-aminophenol and oxalyl chloride following the general procedure $\mathrm{C}$ as a pale yellow solid in $90 \%$ yield. ${ }^{1} \mathrm{H}$ NMR (400 $\mathrm{MHz}, d 6-\mathrm{DMSO}) \delta 9.96(\mathrm{~s}, 2 \mathrm{H}), 9.25(\mathrm{~s}, 2 \mathrm{H}), 6.50(\mathrm{~s}, 4 \mathrm{H}), 2.06(\mathrm{~s}, 12 \mathrm{H}) ;{ }^{13} \mathrm{C} \mathrm{NMR}$ (100 MHz, d6-DMSO) $\delta 159.3,155.9,136.1,125.5,114.3,18.2$; HRMS (DART) calcd. for $\mathrm{C}_{18} \mathrm{H}_{21} \mathrm{~N}_{2} \mathrm{O}_{4}(\mathrm{M}+\mathrm{H})^{+}$329.1496, found: 329.1496 .

To the solution of $\mathbf{L 3}$ ' in DMF (0.5 M) was added $\mathrm{K}_{2} \mathrm{CO}_{3}$ (2.5 eq) and MeI (3.0 eq). After the resulting mixture was stirred at room temperature for $12 \mathrm{~h}$, it was concentrated in vacuo to remove the solvent and water was added to the resulting residue to dissolve inorganic salts. Then the slurry was filtered and the solid on filter paper was washed with water and cold diethyl ether. The residue was dried in vacuo to afford $\mathbf{L 3}$ as a pale yellow solid in $86 \%$ yield. ${ }^{1} \mathrm{H}$ NMR (400 MHz, $\left.d 6-\mathrm{DMSO}\right) \delta$ $10.09(\mathrm{~s}, 2 \mathrm{H}), 6.69(\mathrm{~s}, 4 \mathrm{H}), 3.73(\mathrm{~s}, 6 \mathrm{H}), 2.13(\mathrm{~s}, 12 \mathrm{H}) ;{ }^{13} \mathrm{C} \mathrm{NMR}(100 \mathrm{MHz}, d 6-$ 
DMSO) $\delta$ 159.2, 157.7, 136.3, 127.1, 112.9, 55.1, 18.2; HRMS (DART) calcd. for

$\mathrm{C}_{20} \mathrm{H}_{25} \mathrm{~N}_{2} \mathrm{O}_{4}(\mathrm{M}+\mathrm{H})^{+} 357.1809$, found: 357.1807<smiles>CC(=O)Nc1ccccc1-c1ccccc1</smiles>

The $\mathbf{L} 4^{3}$ was prepared from 2-phenylaniline and oxalyl chloride following the general procedure $\mathrm{C}$ as a white solid in $89 \%$ yield. ${ }^{1} \mathrm{H}$ NMR (400 $\left.\mathrm{MHz}, \mathrm{CDCl}_{3}\right) \delta 9.54(\mathrm{~s}, 2 \mathrm{H})$, $8.38(\mathrm{dd}, J=8.3,1.2 \mathrm{~Hz}, 2 \mathrm{H}), 7.57-7.50(\mathrm{~m}, 4 \mathrm{H}), 7.49-7.43(\mathrm{~m}, 2 \mathrm{H}), 7.43-7.35(\mathrm{~m}$, $6 \mathrm{H})$, 7.34-7.29 (m, 2H), 7.26-7.21 (m, 2H); $\left.{ }^{13} \mathrm{C} \mathrm{NMR} \mathrm{(100} \mathrm{MHz,} \mathrm{CDCl}_{3}\right) \delta$ 157.5, $137.3,133.4,133.0,130.5,129.4,129.3,128.6,128.5,125.4,120.4$.

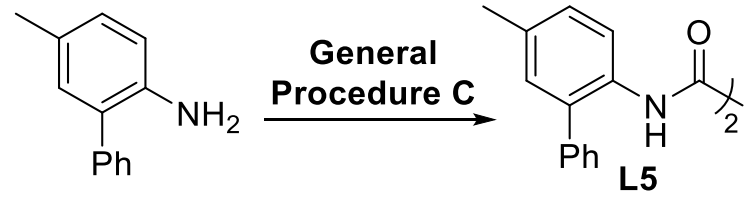

The $\mathbf{L 5}$ was prepared from 2-amino-5-methylbiphenyl ${ }^{4}$ and oxalyl chloride following the general procedure $\mathrm{C}$ as a white solid in $91 \%$ yield. ${ }^{1} \mathrm{H} \mathrm{NMR}\left(400 \mathrm{MHz}, \mathrm{CDCl}_{3}\right) \delta$ $9.47(\mathrm{~s}, 2 \mathrm{H}), 8.25-8.23(\mathrm{~d}, J=8.0 \mathrm{~Hz}, 2 \mathrm{H}), 7.53-7.50(\mathrm{~m}, 4 \mathrm{H}), 7.46-7.42(\mathrm{~m}, 2 \mathrm{H})$, 7.39-7.37 (d, $J=7.6 \mathrm{~Hz}, 4 \mathrm{H}), 7.18-7.16(\mathrm{~d}, J=8.4 \mathrm{~Hz}, 2 \mathrm{H}), 7.12(\mathrm{~s}, 2 \mathrm{H}), 2.36(\mathrm{~s}$, $6 \mathrm{H}) ;{ }^{13} \mathrm{C} \mathrm{NMR}\left(100 \mathrm{MHz}, \mathrm{CDCl}_{3}\right) \delta 157.4,137.5,135.1,133.0,131.1,131.0,129.4$, 129.3, 129.1, 128.4, 120.4, 21.1; ESI-HRMS $m / z$ calcd for $\mathrm{C}_{28} \mathrm{H}_{25} \mathrm{O}_{2} \mathrm{~N}_{2}(\mathrm{M}+\mathrm{H})^{+}$ 421.1911, found 421.1906.

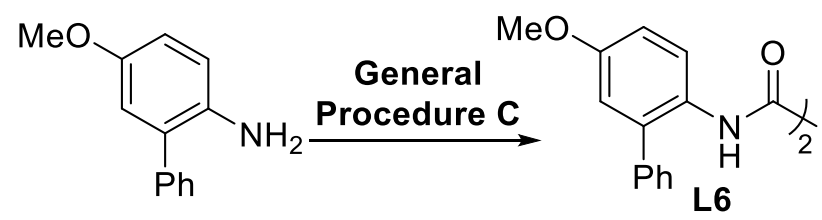

The L6 was prepared from 2-amino-5-methoxybiphenyl ${ }^{4}$ and oxalyl chloride following the general procedure $\mathrm{C}$ as a white solid in $87 \%$ yield. ${ }^{1} \mathrm{H} \mathrm{NMR}(400 \mathrm{MHz}$, 
$\left.\mathrm{CDCl}_{3}\right) \delta 9.37(\mathrm{~s}, 2 \mathrm{H}), 8.26-8.24(\mathrm{~d}, J=8.8 \mathrm{~Hz}, 2 \mathrm{H}), 7.56-7.50(\mathrm{~m}, 4 \mathrm{H}), 7.46-7.43(\mathrm{~m}$, $2 \mathrm{H}), 7.40-7.38(\mathrm{~m}, 4 \mathrm{H}), 6.92-6.89\left(\mathrm{dd}, J_{1}=2.8 \mathrm{~Hz}, J_{2}=8.8 \mathrm{~Hz}, 2 \mathrm{H}\right), 6.85(\mathrm{~d}, J=3.2$ $\mathrm{Hz}, 2 \mathrm{H}), 3.82(\mathrm{~s}, 6 \mathrm{H}) ;{ }^{13} \mathrm{C} \mathrm{NMR}\left(100 \mathrm{MHz}, \mathrm{CDCl}_{3}\right) \delta 157.3,156.9,137.4,134.7$, 129.4, 129.2, 128.6, 126.7, 122.1, 115.8, 113.6, 55.7; ESI-HRMS $\mathrm{m} / \mathrm{z}$ calcd for $\mathrm{C}_{28} \mathrm{H}_{25} \mathrm{O}_{4} \mathrm{~N}_{2}(\mathrm{M}+\mathrm{H})^{+} 453.1809$, found 453.1805.<smiles>CC(=O)Nc1ccccc1Oc1ccccc1</smiles>

The L7 was prepared from 2-phenoxyaniline and oxalyl chloride following the general procedure $\mathrm{C}$ as a white solid in $85 \%$ yield. ${ }^{1} \mathrm{H} \mathrm{NMR}\left(400 \mathrm{MHz}, \mathrm{CDCl}_{3}\right) \delta 9.97$ (s, 2H), 8.47 (dd, $J=7.9,1.8 \mathrm{~Hz}, 2 \mathrm{H}), 7.44-7.32(\mathrm{~m}, 4 \mathrm{H}), 7.21-7.04(\mathrm{~m}, 10 \mathrm{H}), 6.90$ $(\mathrm{dd}, J=8.0,1.6 \mathrm{~Hz}, 2 \mathrm{H}) ;{ }^{13} \mathrm{C} \mathrm{NMR}\left(100 \mathrm{MHz}, \mathrm{CDCl}_{3}\right) \delta 157.5,156.2,146.8,130.1$, $128.1,125.5,124.3,123.9,120.6,119.1,117.7$; ESI-HRMS $\mathrm{m} / z$ calcd. for $\mathrm{C}_{26} \mathrm{H}_{21} \mathrm{~N}_{2} \mathrm{O}_{4}(\mathrm{M}+\mathrm{H})^{+}:$425.1496, found: 425.1492 .<smiles>CCc1cccc(CC)c1NC(=O)O</smiles>

The $\mathbf{L 8}^{5}$ was prepared from 2,6-diethylaniline and oxalyl chloride following the general procedure $\mathrm{C}$ as a white solid in $87 \%$ yield. ${ }^{1} \mathrm{H} \mathrm{NMR}\left(400 \mathrm{MHz}, \mathrm{CDCl}_{3}\right) \delta 8.90$ $(\mathrm{s}, 2 \mathrm{H}), 7.29(\mathrm{dd}, J=8.2,7.0 \mathrm{~Hz}, 2 \mathrm{H}), 7.18(\mathrm{~d}, J=7.6 \mathrm{~Hz}, 4 \mathrm{H}), 2.63(\mathrm{q}, J=7.6 \mathrm{~Hz}$, 8H), $1.22(\mathrm{t}, J=7.6 \mathrm{~Hz}, 12 \mathrm{H}) ;{ }^{13} \mathrm{C} \mathrm{NMR}\left(100 \mathrm{MHz}, \mathrm{CDCl}_{3}\right) \delta$ 159.0, 141.2, 131.3, $128.7,126.7,25.1,14.5$. 
<smiles>[Y]C(=O)Nc1c(CCC)cccc1C(=O)c1ccccc1</smiles>

The $\mathbf{L 9}^{5}$ was prepared from 2,6-diisopropylaniline and oxalyl chloride following the general procedure $\mathrm{C}$ as a white solid in $83 \%$ yield. ${ }^{1} \mathrm{H}$ NMR $\left(400 \mathrm{MHz}, \mathrm{CDCl}_{3}\right) \delta 8.88$ (s, 2H), 7.39-7.32 (m, 2H), 7.25-7.20 (m, 4H), 3.07 (hept, $J=6.8 \mathrm{~Hz}, 4 \mathrm{H}), 1.23$ (d, $J$ $=6.9 \mathrm{~Hz}, 24 \mathrm{H}) ;{ }^{13} \mathrm{C} \mathrm{NMR}\left(100 \mathrm{MHz}, \mathrm{CDCl}_{3}\right) \delta 159.7,145.9,129.8,129.0,123.9$, 29.2, 23.7.

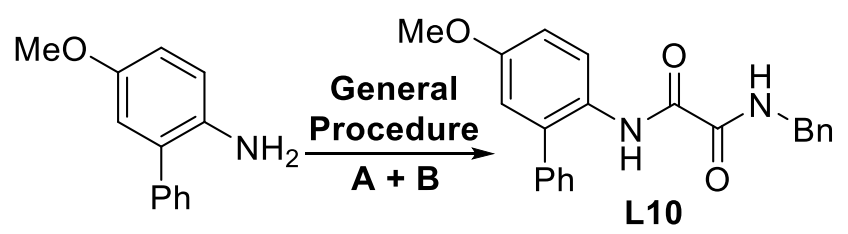

The L10 was prepared from 2-amino-5-methoxybiphenyl ${ }^{4}$, mono-methyl oxalyl chloride and $\mathrm{BnNH}_{2}$ in two steps following the general procedures $\mathrm{A}$ and $\mathrm{B}$ as a white solid in $88 \%$ overall yield. ${ }^{1} \mathrm{H}$ NMR $\left(400 \mathrm{MHz}, \mathrm{CDCl}_{3}\right) \delta 9.35(\mathrm{~s}, 1 \mathrm{H}), 8.30(\mathrm{~d}, J=$ 9.0 Hz, 1H), 7.79 (br s, 1H), 7.55-7.49 (m, 2H), 7.47-7.42 (m, 1H), 7.42-7.37 (m, 2H), 7.37-7.31 (m, 2H), 7.31-7.26 (m, 3H), $6.94(\mathrm{dd}, J=9.0,2.9 \mathrm{~Hz}, 1 \mathrm{H}), 6.87(\mathrm{~d}, J=2.9$ $\mathrm{Hz}, 1 \mathrm{H}), 4.48(\mathrm{~d}, J=6.2 \mathrm{~Hz}, 2 \mathrm{H}), 3.83(\mathrm{~s}, 3 \mathrm{H}) ;{ }^{13} \mathrm{C}$ NMR $(100 \mathrm{MHz}, d 6-\mathrm{DMSO}) \delta$ $159.9,158.1,157.1,138.5,138.0,136.8,128.7,128.6,128.3,127.7,127.3,126.9$, 126.6, 125.3, 115.2, 113.5, 55.4, 42.5; ESI-HRMS $\mathrm{m} / \mathrm{z}$ calcd for $\mathrm{C}_{22} \mathrm{H}_{21} \mathrm{O}_{3} \mathrm{~N}_{2}(\mathrm{M}+$ H) ${ }^{+} 361.1547$, found 361.1545 .

L11 L14 are commercial available.

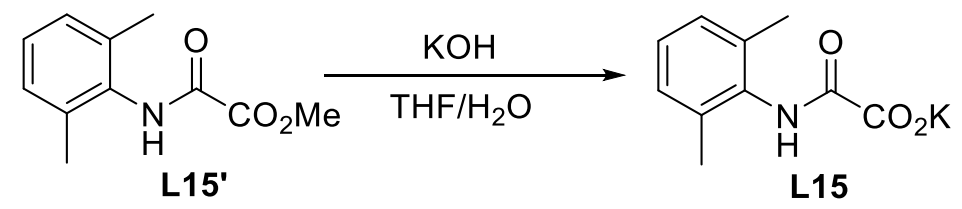


The $\mathbf{L 1 5}^{{ }^{6}}$ was prepared from 2,6-dimethylaniline and mono-methyl oxalyl chloride following the general procedure A as a white solid in $93 \%$ yield. ${ }^{1} \mathrm{H}$ NMR (400 MHz, $\left.\mathrm{CDCl}_{3}\right) \delta 8.41(\mathrm{~s}, 1 \mathrm{H}), 7.21-7.05(\mathrm{~m}, 3 \mathrm{H}), 3.98(\mathrm{~s}, 3 \mathrm{H}), 2.24(\mathrm{~s}, 6 \mathrm{H}) ;{ }^{13} \mathrm{C}$ NMR $(100$ $\left.\mathrm{MHz}, \mathrm{CDCl}_{3}\right) \delta 161.2,154.4,134.9,132.1,128.3,127.9,53.8,18.3$.

To the solution of L15' in THF (0.5 M) was added $\mathrm{KOH}$ (2.0 M aqueous solution, 1.0 eq) and the resulting mixture was stirred at room temperature until L15' was completely consumed as detected via TLC. Then it was concentrated in vacuo to remove THF and water, and oven-dried to afford L15 as a white solid in $98 \%$ yield. ${ }^{1} \mathrm{H}$ NMR (400 MHz, d4-MeOD) $\delta$ 7.13-7.05 (m, 3H), $2.23(\mathrm{~s}, 6 \mathrm{H}) ;{ }^{13} \mathrm{C}$ NMR $(100$ MHz, $d 4-\mathrm{MeOD}) \delta 166.3,165.1,136.6,135.3,129.0,128.3,18.5$.

\section{Preparation of Heteroaryl Chloride 1k}

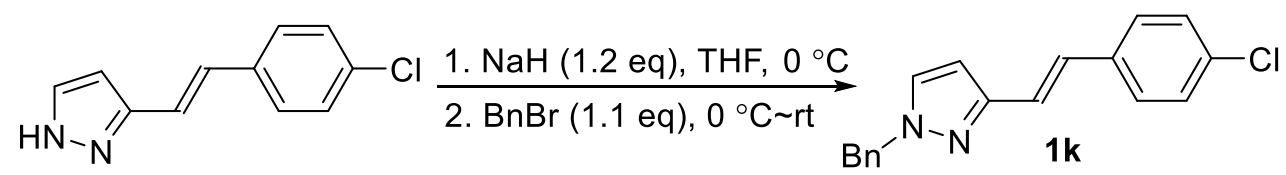

(E)-1-Benzyl-3-(4-chlorostyryl)-1H-pyrazole (1k). NaH (60\%, 144 mg, 3.6 mmol, 1.2 eq) was added to a stirring solution of (E)-3-(4-chlorostyryl)-1H-pyrazole (614 mg, $3.0 \mathrm{mmol}, 1.0 \mathrm{eq})$ in $\mathrm{THF}(15 \mathrm{~mL})$ under $\operatorname{Ar}$ at $0{ }^{\circ} \mathrm{C}$. After the reaction mixture was stirred for $15 \mathrm{~min}, \mathrm{BnBr}(0.4 \mathrm{~mL}, 3.3 \mathrm{mmol}, 1.1 \mathrm{eq})$ was added. Then the reaction mixture was warmed to room temperature and was stirred for $12 \mathrm{~h}$. The reaction mixture was poured into EtOAc/ $\mathrm{H}_{2} \mathrm{O}(20 \mathrm{~mL} / 20 \mathrm{~mL})$. The aqueous layer was extracted with EtOAc $(20 \mathrm{~mL})$, and the combined organic layers were washed with saturated aqueous $\mathrm{NaCl}$, dried $\left(\mathrm{Na}_{2} \mathrm{SO}_{4}\right)$ and concentrated on a rotary evaporator. The resulting residue was purified by silica gel flash chromatography to afford $\mathbf{1 k}$ as white 
solid (584 mg, 66\%). ${ }^{1} \mathrm{H}$ NMR $\left(500 \mathrm{MHz}, \mathrm{CDCl}_{3}\right) \delta 7.41(\mathrm{~d}, J=8.5 \mathrm{~Hz}, 2 \mathrm{H}), 7.38-$ $7.29(\mathrm{~m}, 6 \mathrm{H}), 7.25-7.21(\mathrm{~m}, 2 \mathrm{H}), 7.12(\mathrm{~d}, J=16.5 \mathrm{~Hz}, 1 \mathrm{H}), 7.04(\mathrm{~d}, J=16.4 \mathrm{~Hz}, 1 \mathrm{H})$, $6.48(\mathrm{~d}, J=2.4 \mathrm{~Hz}, 1 \mathrm{H}), 5.31(\mathrm{~s}, 2 \mathrm{H}) ;{ }^{13} \mathrm{C} \mathrm{NMR}\left(125 \mathrm{MHz}, \mathrm{CDCl}_{3}\right) \delta 150.6,136.5$, $135.9,133.2,130.7,129.0,128.9,128.4,128.2,127.7,127.6,121.6,103.6,56.2$; ESIHRMS $m / z$ calcd. for $\mathrm{C}_{18} \mathrm{H}_{16} \mathrm{~N}_{2} \mathrm{Cl}(\mathrm{M}+\mathrm{H})^{+} 295.0997$, found: 295.0999 .

\section{General procedure for the CuI-catalyzed monoarylation with ammonia}

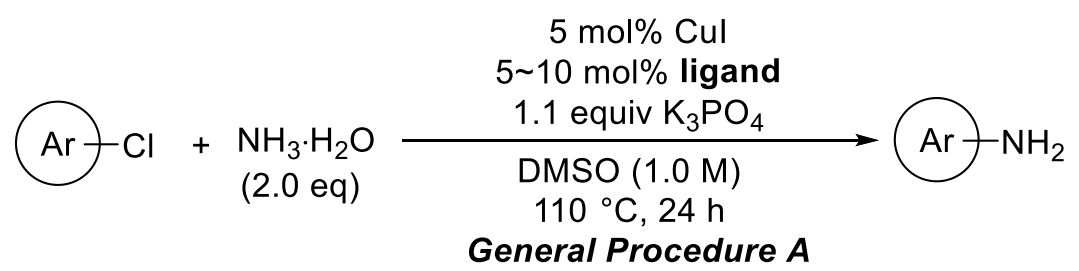

General procedure A: An $10 \mathrm{~mL}$ resealable screw-cap test tube equipped with a Teflon-coated magnetic stir bar was charged with $\mathrm{CuI}(9.5 \mathrm{mg}, 0.05 \mathrm{mmol})$, ligand (0.05-0.1 mmol), (hetero)aryl halide (if solid) $(1.0 \mathrm{mmol})$ and $\mathrm{K}_{3} \mathrm{PO}_{4}(233 \mathrm{mg}, 1.1$ mmol). The tube was then evacuated and backfilled with argon (this sequence was repeated a total of three times), and ammonia aqueous solution (w/w 25\%, $160 \mu \mathrm{L}$, $2.0 \mathrm{mmol})$, (hetero)aryl halide (if liquid) $(1.0 \mathrm{mmol})$ and DMSO $(1.0 \mathrm{~mL})$ were then added into the tube via syringe. The reaction mixture was stirred at $110{ }^{\circ} \mathrm{C}$ in an oil bath for $24 \mathrm{~h}$. After cooling to room temperature, the crude product was diluted with ethyl acetate $(\sim 40 \mathrm{~mL})$, and transferred into a separating funnel, and brine $(\sim 30 \mathrm{~mL})$ was added. The organic phase was separated and aqueous phase was extracted with ethyl acetate twice. The combined organic phase was dried over $\mathrm{Na}_{2} \mathrm{SO}_{4}$, and concentrated in vacuo. The residue was purified by silica gel flash chromatography using a solvent mixture (ethyl acetate/hexanes, diethyl ether/hexanes, or 
dichloromethane/methanol) as an eluent to afford the corresponding (hetero)aryl amine products.

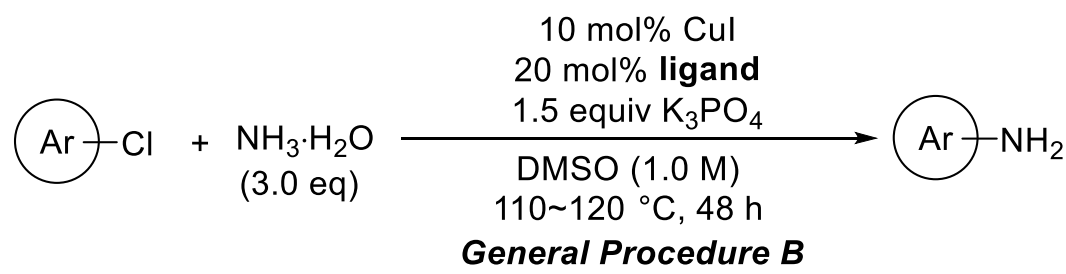

General procedure $\mathbf{B}$ is the same as general procedure $\mathbf{A}$, except for adding $\mathrm{CuI}$ (19.0 mg, $0.1 \mathrm{mmol})$, ligand $(0.2 \mathrm{mmol}), \mathrm{K}_{3} \mathrm{PO}_{4}(318 \mathrm{mg}, 1.5 \mathrm{mmol})$ and ammonia aqueous solution (w/w 25\%, $238 \mu \mathrm{L}, 3.0 \mathrm{mmol}$ ) and using higher reaction temperatures $\left(110 \sim 120{ }^{\circ} \mathrm{C}\right)$ and a longer reaction time $(48 \mathrm{~h})$.

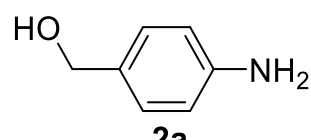

Following the general procedure A, compound $\mathbf{2 a}^{7}$ was prepared from 4-chlorobenzyl alcohol as yellow solid (106 mg, 87\% yield). ${ }^{1} \mathrm{H}$ NMR (400 MHz, $\left.\mathrm{CDCl}_{3}\right) \delta 7.16(\mathrm{~d}, J$ $=8.3 \mathrm{~Hz}, 2 \mathrm{H}), 6.67(\mathrm{~d}, J=8.4 \mathrm{~Hz}, 2 \mathrm{H}), 4.55(\mathrm{~s}, 2 \mathrm{H}), 3.67(\mathrm{br} \mathrm{s}, 2 \mathrm{H}) ;{ }^{13} \mathrm{C}$ NMR $(100$ $\left.\mathrm{MHz}, \mathrm{CDCl}_{3}\right) \delta 146.1,131.2,128.8,115.2,65.3$.<smiles>CC(C)(C)C1=[C+]C=C(N)C=C1</smiles>

Following the general procedure A, compound $\mathbf{2} \mathbf{b}^{8}$ was prepared from 4-chloro-tertbutylbenzene as yellow liquid (119 mg, 80\% yield). ${ }^{1} \mathrm{H}$ NMR (400 $\left.\mathrm{MHz}, \mathrm{CDCl}_{3}\right) \delta$ $7.21(\mathrm{~d}, J=8.6 \mathrm{~Hz}, 2 \mathrm{H}), 6.67(\mathrm{~d}, J=8.1 \mathrm{~Hz}, 2 \mathrm{H}), 3.57(\mathrm{br} \mathrm{s}, 2 \mathrm{H}), 1.31(\mathrm{~s}, 9 \mathrm{H}) ;{ }^{13} \mathrm{C}$ NMR (100 MHz, $\left.\mathrm{CDCl}_{3}\right) \delta 143.8,141.1,125.9,114.9,33.8,31.5$.<smiles>CCCCOc1ccc(N)cc1</smiles> 
Following the general procedure A, compound $2 \mathbf{c}^{9}$ was prepared from 4-tertbutoxyphenyl chloride as pale brown solid (135 mg, 82\% yield). ${ }^{1} \mathrm{H}$ NMR (400 MHz, $\left.\mathrm{CDCl}_{3}\right) \delta$ 6.84-6.75 (m, 2H), 6.63-6.55 (m, 2H), $3.51(\mathrm{~s}, 2 \mathrm{H}), 1.28(\mathrm{~s}, 9 \mathrm{H}) ;{ }^{13} \mathrm{C} \mathrm{NMR}$ $\left(100 \mathrm{MHz}, \mathrm{CDCl}_{3}\right) \delta 147.1,142.2,125.3,115.4,77.7,28.7$.<smiles>CSc1ccc(N)cc1</smiles>

Following the general procedure A, compound $\mathbf{2 d}^{8}$ was prepared from (4chlorophenyl)trimethylsilane as light yellow liquid (105 mg, 64\% yield). ${ }^{1} \mathrm{H}$ NMR $\left(400 \mathrm{MHz}, \mathrm{CDCl}_{3}\right) \delta 7.33(\mathrm{~d}, J=8.4 \mathrm{~Hz}, 2 \mathrm{H}), 6.70(\mathrm{~d}, J=8.4 \mathrm{~Hz}, 2 \mathrm{H}), 3.65(\mathrm{br} \mathrm{s}, 2 \mathrm{H})$, $0.23(\mathrm{~s}, 9 \mathrm{H}) ;{ }^{13} \mathrm{C} \mathrm{NMR}\left(100 \mathrm{MHz}, \mathrm{CDCl}_{3}\right) \delta 147.1,134.6,128.4,114.7,-0.8$.<smiles>CSc1ccc(N)cc1</smiles>

Following the general procedure A, compound $\mathbf{2 e}^{8}$ was prepared from (4chlorophenyl)methylsulfide as brown liquid (118 mg, 85\% yield). ${ }^{1} \mathrm{H}$ NMR (400 MHz, $\left.\mathrm{CDCl}_{3}\right) \delta 7.18(\mathrm{~d}, J=8.5 \mathrm{~Hz}, 2 \mathrm{H}), 6.63(\mathrm{~d}, J=8.5 \mathrm{~Hz}, 2 \mathrm{H}), 3.53(\mathrm{~s}, 2 \mathrm{H}), 2.41(\mathrm{~s}, 3 \mathrm{H})$; ${ }^{13} \mathrm{C}$ NMR $\left(125 \mathrm{MHz}, \mathrm{CDCl}_{3}\right) \delta 145.2,131.0,125.7,115.7,18.8$.<smiles>Nc1ccc(C(=O)N2CCCCC2)cc1</smiles>

Following the general procedure A, compound $\mathbf{2 f}^{8}$ was prepared from (4chlorophenyl)(piperidin-1-yl)methanone as pale yellow solid (176 mg, 86\% yield). ${ }^{1} \mathrm{H}$ NMR (400 MHz, $\left.\mathrm{CDCl}_{3}\right) \delta 7.24(\mathrm{~d}, J=8.5 \mathrm{~Hz}, 2 \mathrm{H}), 6.64(\mathrm{~d}, J=8.5 \mathrm{~Hz}, 2 \mathrm{H}), 3.83(\mathrm{br}$ s, 2H), 3.53 (br s, 4H), 1.72-1.62 (m, 2H), 1.57 (br s, 4H); ${ }^{13} \mathrm{C}$ NMR (100 MHz, $\left.\mathrm{CDCl}_{3}\right) \delta 170.6,148.3,128.7,124.5,113.6,25.8,24.3$. 
<smiles>Nc1ccc(-c2nc3ccccc3s2)cc1</smiles>

Following the general procedure A, compound $\mathbf{2 g}^{8}$ was prepared from 2-(4chlorophenyl)benzothiazole as pale yellow solid (215 mg, 95\% yield). ${ }^{1} \mathrm{H}$ NMR (400 $\left.\mathrm{MHz}, \mathrm{CDCl}_{3}\right) \delta 7.99(\mathrm{~d}, J=8.1 \mathrm{~Hz}, 1 \mathrm{H}), 7.93-7.87(\mathrm{~m}, 2 \mathrm{H}), 7.85(\mathrm{~d}, J=7.9 \mathrm{~Hz}, 1 \mathrm{H})$, 7.48-7.41 (m, 1H), 7.36-7.29 (m, 1H), 6.77-6.71 (m, 2H), $4.00(\mathrm{~s}, 2 \mathrm{H}) ;{ }^{13} \mathrm{C}$ NMR $(125$ $\left.\mathrm{MHz}, \mathrm{CDCl}_{3}\right) \delta 168.7,154.4,149.4,134.7,129.3,126.2,124.6,124.1,122.6,121.6$, 114.9 .<smiles>Nc1ccc(-n2ccnc2)cc1</smiles>

$2 \mathrm{~h}$

Following the general procedure A, compound $\mathbf{2 h}^{8}$ was prepared from 1-(4chlorophenyl)imidazole as yellow solid (140 mg, 88\% yield). ${ }^{1} \mathrm{H}$ NMR (400 MHz, $\left.\mathrm{CDCl}_{3}\right) \delta 7.70(\mathrm{~s}, 1 \mathrm{H}), 7.16-7.12(\mathrm{~m}, 3 \mathrm{H}), 7.12-7.09(\mathrm{~m}, 1 \mathrm{H}), 6.74-6.67(\mathrm{~m}, 2 \mathrm{H}), 3.69$ $(\mathrm{s}, 2 \mathrm{H}) ;{ }^{13} \mathrm{C} \mathrm{NMR}\left(100 \mathrm{MHz}, \mathrm{CDCl}_{3}\right) \delta 146.3,135.9,129.7,128.8,123.4,119.0$, 115.6

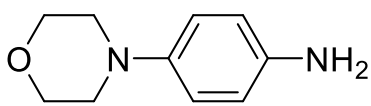

2i

Following the general procedure A, compound $\mathbf{2} \mathbf{i}^{8}$ was prepared from 4-(4-chlorophenyl)morpholine as taupe solid (147 mg, 83\% yield). ${ }^{1} \mathrm{H}$ NMR (400 MHz, $\left.\mathrm{CDCl}_{3}\right) \delta$ 6.83-6.76 (m, 2H), 6.70-6.63 (m, 2H), 3.89-3.81 (m, 4H), $3.43(\mathrm{~s}, 2 \mathrm{H}), 3.08-2.95(\mathrm{~m}$, $4 \mathrm{H}) ;{ }^{13} \mathrm{C}$ NMR $\left(125 \mathrm{MHz}, \mathrm{CDCl}_{3}\right) \delta 144.6,140.4,118.3,116.4,67.2,51.3$.<smiles>Nc1ccc(-n2cccc2)cc1</smiles> 
Following the general procedure A, compound $\mathbf{2 j}^{8}$ was prepared from 4pyrrolylchlorobenzene as brownish yellow solid (95 mg, 60\% yield). ${ }^{1} \mathrm{H}$ NMR (400 $\left.\mathrm{MHz}, \mathrm{CDCl}_{3}\right) \delta$ 7.15-7.06 (m, 2H), 6.94-6.86 (m, 2H), 6.69-6.60 (m, 2H), 6.27-6.18 $(\mathrm{m}, 2 \mathrm{H}), 3.60(\mathrm{~s}, 2 \mathrm{H}) ;{ }^{13} \mathrm{C} \mathrm{NMR}\left(125 \mathrm{MHz}, \mathrm{CDCl}_{3}\right) \delta 144.7,133.0,122.5,119.8$, 115.8, 109.6.

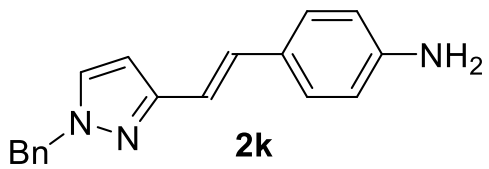

Following the general procedure A, compound $\mathbf{2 k}$ was prepared from $(E)$-1-benzyl-3(4-chlorostyryl)-1H-pyrazole (1k) as yellow solid (245 mg, 89\% yield). ${ }^{1} \mathrm{H}$ NMR (400 $\left.\mathrm{MHz}, \mathrm{CDCl}_{3}\right) \delta 7.39-7.26(\mathrm{~m}, 6 \mathrm{H}), 7.25-7.18(\mathrm{~m}, 2 \mathrm{H}), 7.06-6.90(\mathrm{~m}, 2 \mathrm{H}), 6.66(\mathrm{~d}, J=$ $8.5 \mathrm{~Hz}, 2 \mathrm{H}), 6.44(\mathrm{~d}, J=2.4 \mathrm{~Hz}, 1 \mathrm{H}), 5.30(\mathrm{~s}, 2 \mathrm{H}), 3.72(\mathrm{br} \mathrm{s}, 2 \mathrm{H}) ;{ }^{13} \mathrm{C}$ NMR $(100$ $\left.\mathrm{MHz}, \mathrm{CDCl}_{3}\right) \delta 151.3,146.2,136.5,130.4,129.8,128.7,127.8,127.5,127.4,116.9$, 115.0, 102.7, 55.7; ESI-HRMS $\mathrm{m} / z$ calcd. for $\mathrm{C}_{18} \mathrm{H}_{18} \mathrm{~N}_{3}(\mathrm{M}+\mathrm{H})^{+}$: 276.1501, found: 276.1497.<smiles>Nc1ccc2sc3ccccc3c(=O)c2c1</smiles>

Following the general procedure A, compound $2 \mathbf{l}^{8}$ was prepared from 2chlorothioxanthen-9-one as yellow solid (160 mg, 70\% yield). ${ }^{1} \mathrm{H}$ NMR (400 MHz, $d 6-\mathrm{DMSO}) \delta 8.44(\mathrm{dd}, J=8.2,1.1 \mathrm{~Hz}, 1 \mathrm{H}), 7.80-7.74(\mathrm{~m}, 1 \mathrm{H}), 7.74-7.67(\mathrm{~m}, 1 \mathrm{H})$, $7.66(\mathrm{~d}, J=2.6 \mathrm{~Hz}, 1 \mathrm{H}), 7.55-7.46(\mathrm{~m}, 2 \mathrm{H}), 7.09(\mathrm{dd}, J=8.6,2.6 \mathrm{~Hz}, 1 \mathrm{H}), 5.67(\mathrm{~s}$, $2 \mathrm{H}) ;{ }^{13} \mathrm{C}$ NMR (125 MHz, d6-DMSO) $\delta 178.7,148.1,137.2,132.1,129.4,129.0$, $127.9,127.1,126.3,125.9,122.1,121.2,110.9$. 
<smiles>COc1cc(N)cc(OC)c1</smiles>

Following the general procedure A, compound $\mathbf{2} \mathbf{m}^{10}$ was prepared from 5-chloro-1,3dimethoxybenzene as light yellow liquid (135 mg, 88\% yield). ${ }^{1} \mathrm{H}$ NMR (400 MHz, $\left.\mathrm{CDCl}_{3}\right) \delta 5.93(\mathrm{t}, J=2.1 \mathrm{~Hz}, 1 \mathrm{H}), 5.87(\mathrm{~d}, J=2.1 \mathrm{~Hz}, 2 \mathrm{H}), 3.74(\mathrm{~s}, 6 \mathrm{H}) ;{ }^{13} \mathrm{C} \mathrm{NMR}$ $\left(100 \mathrm{MHz}, \mathrm{CDCl}_{3}\right) \delta 161.6,148.5,93.6,90.8,55.0$.<smiles>Nc1ccc2c(c1)OCO2</smiles>

Following the general procedure A, compound $\mathbf{2 n}^{8}$ was prepared from 4-chloro-1,2(methylenedioxy)benzene as dark brown solid (112 mg, 82\% yield). ${ }^{1} \mathrm{H}$ NMR (400 $\left.\mathrm{MHz}, \mathrm{CDCl}_{3}\right) \delta 6.62(\mathrm{~d}, J=8.1 \mathrm{~Hz}, 1 \mathrm{H}), 6.29(\mathrm{~d}, J=2.3 \mathrm{~Hz}, 1 \mathrm{H}), 6.13(\mathrm{dd}, J=8.1$, $2.3 \mathrm{~Hz}, 1 \mathrm{H}), 5.86(\mathrm{~s}, 2 \mathrm{H}), 3.45(\mathrm{br} \mathrm{s}, 2 \mathrm{H}) ;{ }^{13} \mathrm{C} \mathrm{NMR}\left(100 \mathrm{MHz}, \mathrm{CDCl}_{3}\right) \delta$ 148.2, $141.5,140.3,108.6,106.9,100.7,98.1$.<smiles>Nc1ccc(Cl)cc1-c1cc(N)c([N+](=O)[O-])cc1F</smiles>

Following the general procedure A, compound $\mathbf{2 0}^{8}$ was prepared from 4-chloro-2fluoro-aniline as brown solid (95 mg, 75\% yield). ${ }^{1} \mathrm{H}$ NMR $\left(400 \mathrm{MHz}, \mathrm{CDCl}_{3}\right) \delta 6.63$ $(\mathrm{dd}, J=9.8,8.3 \mathrm{~Hz}, 1 \mathrm{H}), 6.42(\mathrm{dd}, J=12.3,2.5 \mathrm{~Hz}, 1 \mathrm{H}), 6.33(\mathrm{ddd}, J=8.3,2.5,1.0$ $\mathrm{Hz}, 1 \mathrm{H}), 3.35(\mathrm{~s}, 4 \mathrm{H}) ;{ }^{13} \mathrm{C} \mathrm{NMR}\left(125 \mathrm{MHz}, \mathrm{CDCl}_{3}\right) \delta 152.6(\mathrm{~d}, J=238.0 \mathrm{~Hz}), 139.4$ $(\mathrm{d}, J=9.4 \mathrm{~Hz}), 126.0(\mathrm{~d}, J=13.5 \mathrm{~Hz}), 118.6(\mathrm{~d}, J=4.8 \mathrm{~Hz}), 111.6(\mathrm{~d}, J=3.2 \mathrm{~Hz})$, $103.6(\mathrm{~d}, J=22.3 \mathrm{~Hz})$. 
<smiles>COc1cc(N)cc(F)c1</smiles>

Following the general procedure A, compound $\mathbf{2} \mathbf{p}^{8}$ was prepared from 1-chloro-3fluoro-5-methoxybenzene as orange red oil (112 mg, 79\% yield). ${ }^{1} \mathrm{H}$ NMR (400 MHz, $\left.\mathrm{CDCl}_{3}\right) \delta$ 6.07-5.98 (m, 3H), $3.75(\mathrm{~s}, 2 \mathrm{H}), 3.74(\mathrm{~s}, 3 \mathrm{H}) ;{ }^{13} \mathrm{C} \mathrm{NMR}\left(125 \mathrm{MHz}, \mathrm{CDCl}_{3}\right)$ $\delta 164.69(\mathrm{~d}, J=241.8 \mathrm{~Hz}), 161.83(\mathrm{~d}, J=13.5 \mathrm{~Hz}), 148.70(\mathrm{~d}, J=13.4 \mathrm{~Hz}), 96.68(\mathrm{~d}$, $J=2.4 \mathrm{~Hz}), 95.05(\mathrm{~d}, J=25.2 \mathrm{~Hz}), 91.96(\mathrm{~d}, J=25.7 \mathrm{~Hz}), 55.46$.<smiles>COc1cc(N)cc(Cl)c1</smiles>

Following the general procedure A, compound $\mathbf{2 q ^ { 1 1 }}$ was prepared from 3,5dichloroanisole as yellow liquid (120 mg, $76 \%$ yield).

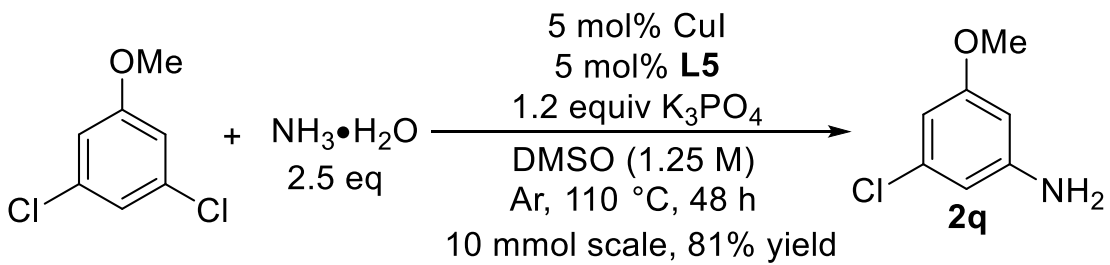

(1.26 g product was obtained in one pot)

In a gram scale preparation, 3,5-dichloroanisole (1.770 g, $10 \mathrm{mmol})$, ammonia aqueous solution (w/w 25\%, $1.9 \mathrm{~mL}, 25 \mathrm{mmol}), \mathrm{CuI}$ (95 mg, $0.5 \mathrm{mmol})$, L5 (210 mg, $0.5 \mathrm{mmol}), \mathrm{K}_{3} \mathrm{PO}_{4}(2.547 \mathrm{~g}, 12 \mathrm{mmol})$ and $\mathrm{DMSO}(8.0 \mathrm{~mL}, 1.25 \mathrm{M})$ were applied to afford compound $2 q(1.264 \mathrm{~g})$ in $81 \%$ yield. ${ }^{1} \mathrm{H} \mathrm{NMR}\left(500 \mathrm{MHz}, \mathrm{CDCl}_{3}\right) \delta 6.32(\mathrm{t}, J$ $=2.0 \mathrm{~Hz}, 1 \mathrm{H}), 6.28(\mathrm{t}, J=1.9 \mathrm{~Hz}, 1 \mathrm{H}), 6.09(\mathrm{t}, J=2.1 \mathrm{~Hz}, 1 \mathrm{H}), 3.73(\mathrm{~s}, 3 \mathrm{H}), 3.63(\mathrm{br}$ s, $2 \mathrm{H}) ;{ }^{13} \mathrm{C} \mathrm{NMR}\left(125 \mathrm{MHz}, \mathrm{CDCl}_{3}\right) \delta 161.3,148.5,135.4,108.1,104.5,99.4,55.39$. 
(Note: A $50 \mathrm{~mL}$ sealed tube was used. Diamination product 5-methoxybenzene-1,3diamine was also isolated in about $10 \%$ yield.)<smiles>Cc1cc(Cl)c(C(F)(F)F)cc1N</smiles>

Following the general procedure A, compound $2 \mathbf{r}^{8}$ was prepared from 1,4-dichloro-2trifluoromethylbenzene as yellow liquid (163 mg, 84\% yield). ${ }^{1} \mathrm{H}$ NMR (400 MHz, $\left.\mathrm{CDCl}_{3}\right) \delta 7.23(\mathrm{~d}, J=8.8 \mathrm{~Hz}, 1 \mathrm{H}), 6.96(\mathrm{~d}, J=2.8 \mathrm{~Hz}, 1 \mathrm{H}), 6.73(\mathrm{dd}, J=8.6,2.7 \mathrm{~Hz}$, 1H), 3.84 (br s, $2 \mathrm{H}) ;{ }^{13} \mathrm{C}$ NMR (125 MHz, $\left.\mathrm{CDCl}_{3}\right) \delta 145.3,132.2,128.8(\mathrm{q}, J=31.0$ $\mathrm{Hz}), 123.0(\mathrm{q}, J=273.1 \mathrm{~Hz}), 120.3(\mathrm{q}, J=1.9 \mathrm{~Hz}), 118.8,113.8(\mathrm{q}, J=5.6 \mathrm{~Hz})$.<smiles>NCc1ccc(N)c(N)c1</smiles>

Following the general procedure A, compound $2 \mathbf{s}^{12}$ was prepared from 2-chloroaniline as brown solid (46 mg, 43\% yield). ${ }^{1} \mathrm{H} \mathrm{NMR}\left(400 \mathrm{MHz}, \mathrm{CDCl}_{3}\right) \delta 6.78-6.66(\mathrm{~m}, 4 \mathrm{H})$, 3.39 (br s, $4 \mathrm{H}) ;{ }^{13} \mathrm{C}$ NMR $\left(100 \mathrm{MHz}, \mathrm{CDCl}_{3}\right) \delta 134.7,120.3,116.8$.<smiles>Cc1ccccc1N</smiles>

Following the general procedure A, L1 (10 mol\%) was used as ligand and the reaction was conducted at $120{ }^{\circ} \mathrm{C}$ for $48 \mathrm{~h}$, compound $\mathbf{2 t}^{8}$ was prepared from 2-chlorotoluene as light brown liquid (61 mg, 58\% yield). ${ }^{1} \mathrm{H}$ NMR (400 MHz, $\left.\mathrm{CDCl}_{3}\right) \delta 7.14-7.02$ (m, 2H), 6.80-6.64 (m, 2H), $3.46(\mathrm{~s}, 2 \mathrm{H}), 2.21(\mathrm{~s}, 3 \mathrm{H}) ;{ }^{13} \mathrm{C} \mathrm{NMR}\left(100 \mathrm{MHz}, \mathrm{CDCl}_{3}\right)$ $\delta 144.5,130.4,127.0,122.4,118.7,115.0,17.4$. 
<smiles>COc1ccc(N)c(C)c1</smiles>

Following the general procedure A, L1 (10 mol\%) was used as ligand and the reaction was conducted at $120{ }^{\circ} \mathrm{C}$ for $48 \mathrm{~h}$, compound $2 \mathbf{u}^{13}$ was prepared from 4-chloro-3methylanisole as brown liquid (87 mg, 64\% yield). ${ }^{1} \mathrm{H}$ NMR $\left(400 \mathrm{MHz}, \mathrm{CDCl}_{3}\right) \delta$ $6.71-6.59(\mathrm{~m}, 3 \mathrm{H}), 3.74(\mathrm{~s}, 3 \mathrm{H}), 2.18(\mathrm{~s}, 3 \mathrm{H}) ;{ }^{13} \mathrm{C} \mathrm{NMR}\left(100 \mathrm{MHz}, \mathrm{CDCl}_{3}\right) \delta$ 152.7, $138.2,124.0,116.3,116.0,112.1,55.7,17.7$.<smiles>COc1ccc(F)cc1N</smiles>
2v

Following the general procedure A, L1 (10 mol\%) was used as ligand and the reaction was conducted at $120{ }^{\circ} \mathrm{C}$ for $48 \mathrm{~h}$, compound $\mathbf{2} \mathbf{v}^{14}$ was prepared from 2-chloro-4fluoroanisole as light brown liquid (105 mg, 75\% yield). ${ }^{1} \mathrm{H} \mathrm{NMR}\left(400 \mathrm{MHz}, \mathrm{CDCl}_{3}\right.$ ) $\delta 6.67(\mathrm{dd}, J=8.8,5.0 \mathrm{~Hz}, 1 \mathrm{H}), 6.45(\mathrm{dd}, J=9.8,3.0 \mathrm{~Hz}, 1 \mathrm{H}), 6.39(\mathrm{td}, J=8.6,3.0$ $\mathrm{Hz}, 1 \mathrm{H}), 3.82(\mathrm{~s}, 3 \mathrm{H}) ;{ }^{13} \mathrm{C} \mathrm{NMR}\left(100 \mathrm{MHz}, \mathrm{CDCl}_{3}\right) \delta 157.7(\mathrm{~d}, J=236.0 \mathrm{~Hz}), 143.4$ $(\mathrm{d}, J=2.1 \mathrm{~Hz}), 137.5(\mathrm{~d}, J=11.0 \mathrm{~Hz}), 110.8(\mathrm{~d}, J=10.0 \mathrm{~Hz}), 103.3(\mathrm{~d}, J=22.9 \mathrm{~Hz})$, $102.1(\mathrm{~d}, J=26.7 \mathrm{~Hz}), 56.0$.<smiles>Nc1ccc2cccnc2c1N</smiles>

Following the general procedure A, L1 (10 mol\%) was used as ligand and the reaction was conducted at $120{ }^{\circ} \mathrm{C}$ for $48 \mathrm{~h}$, compound $\mathbf{2} \mathbf{w}^{15}$ was prepared from 7 -chloro-8methylquinoline as yellow solid $(110 \mathrm{mg}, 70 \%$ yield $) .{ }^{1} \mathrm{H}$ NMR $\left(400 \mathrm{MHz}, \mathrm{CDCl}_{3}\right) \delta$ $8.83(\mathrm{~d}, J=4.3 \mathrm{~Hz}, 1 \mathrm{H}), 7.98(\mathrm{dd}, J=8.1,2.1 \mathrm{~Hz}, 1 \mathrm{H}), 7.51(\mathrm{~d}, J=8.7 \mathrm{~Hz}, 1 \mathrm{H}), 7.15$ 
$(\mathrm{dd}, J=8.0,4.3 \mathrm{~Hz}, 1 \mathrm{H}), 7.01(\mathrm{~d}, J=8.7 \mathrm{~Hz}, 1 \mathrm{H}), 4.03(\mathrm{br} \mathrm{s}, 2 \mathrm{H}), 2.60(\mathrm{~s}, 3 \mathrm{H}) ;{ }^{13} \mathrm{C}$

NMR $\left(100 \mathrm{MHz}, \mathrm{CDCl}_{3}\right) \delta 149.5,148.2,145.0,136.1,126.2,122.5,118.5,117.2$, $114.8,10.3$.<smiles></smiles>

Following the general procedure A, compound $2 \mathbf{x}^{16}$ was prepared from 2-chloro-4methyl-quinoline as yellow solid (138 mg, 87\% yield). ${ }^{1} \mathrm{H}$ NMR (400 MHz, $\left.\mathrm{CDCl}_{3}\right) \delta$ $7.79(\mathrm{dd}, J=8.3,1.4 \mathrm{~Hz}, 1 \mathrm{H}), 7.67(\mathrm{dd}, J=8.3,1.2 \mathrm{~Hz}, 1 \mathrm{H}), 7.56(\mathrm{~d}, J=1.5 \mathrm{~Hz}, 1 \mathrm{H})$, $7.35-7.23(\mathrm{~m}, 1 \mathrm{H}), 6.59(\mathrm{~s}, 1 \mathrm{H}), 4.94(\mathrm{br} \mathrm{s}, 2 \mathrm{H}), 2.58(\mathrm{~d}, J=1.0 \mathrm{~Hz}, 3 \mathrm{H}) ;{ }^{13} \mathrm{C} \mathrm{NMR}$ $\left(125 \mathrm{MHz}, \mathrm{CDCl}_{3}\right) \delta 155.9,148.3,142.4,130.4,123.9,123.2,122.7,122.6,112.4$, 18.8.<smiles></smiles>

Following the general procedure A, compound $\mathbf{2 y}{ }^{8}$ was prepared from 3chloroquinoline as pale yellow solid (133 mg, 93\% yield). ${ }^{1} \mathrm{H}$ NMR (500 MHz, $\left.\mathrm{CDCl}_{3}\right) \delta 8.50(\mathrm{~d}, J=2.8 \mathrm{~Hz}, 1 \mathrm{H}), 8.00-7.93(\mathrm{~m}, 1 \mathrm{H}), 7.62-7.55(\mathrm{~m}, 1 \mathrm{H}), 7.47-7.38$ $(\mathrm{m}, 2 \mathrm{H}), 7.22(\mathrm{~d}, J=2.7 \mathrm{~Hz}, 1 \mathrm{H}), 3.83($ br s, $2 \mathrm{H}) ;{ }^{13} \mathrm{C} \mathrm{NMR}\left(100 \mathrm{MHz}, \mathrm{CDCl}_{3}\right) \delta$ $143.1,142.5,140.0,129.2,128.9,126.9,125.9,125.5,114.8$.<smiles></smiles>

Following the general procedure A, compound $\mathbf{2 z}^{8}$ was prepared from 6chloroquinoline as yellow solid (140 mg, 96\% yield). ${ }^{1} \mathrm{H}$ NMR (400 $\left.\mathrm{MHz}, \mathrm{CDCl}_{3}\right) \delta$ 
$8.65(\mathrm{dd}, J=4.2,1.6 \mathrm{~Hz}, 1 \mathrm{H}), 7.94-7.86(\mathrm{~m}, 2 \mathrm{H}), 7.27(\mathrm{dd}, J=8.2,4.3 \mathrm{~Hz}, 1 \mathrm{H}), 7.16$ $(\mathrm{dd}, J=8.9,2.6 \mathrm{~Hz}, 1 \mathrm{H}), 6.90(\mathrm{~d}, J=2.7 \mathrm{~Hz}, 1 \mathrm{H}), 3.96(\mathrm{br} \mathrm{s}, 2 \mathrm{H}) ;{ }^{13} \mathrm{C}$ NMR $(100$ $\left.\mathrm{MHz}, \mathrm{CDCl}_{3}\right) \delta 146.5,144.9,143.1,133.8,130.2,129.7,121.6,121.3,107.2$.<smiles>Cc1ccc2ccc(N)cc2n1</smiles>

Following the general procedure A, compound $\mathbf{2} \mathbf{a a}^{17}$ was prepared from 7-chloro-2methylquinoline as yellowish-green solid (152 mg, 96\% yield). ${ }^{1} \mathrm{H}$ NMR (400 MHz, $\left.\mathrm{CDCl}_{3}\right) \delta 7.87(\mathrm{~d}, J=8.3 \mathrm{~Hz}, 1 \mathrm{H}), 7.56(\mathrm{~d}, J=8.6 \mathrm{~Hz}, 1 \mathrm{H}), 7.15(\mathrm{~d}, J=2.3 \mathrm{~Hz}, 1 \mathrm{H})$, $7.02(\mathrm{~d}, J=8.2 \mathrm{~Hz}, 1 \mathrm{H}), 6.91(\mathrm{dd}, J=8.6,2.3 \mathrm{~Hz}, 1 \mathrm{H}), 4.04(\mathrm{br} \mathrm{s}, 2 \mathrm{H}), 2.67$ (s, 3H);

${ }^{13} \mathrm{C}$ NMR $\left(100 \mathrm{MHz}, \mathrm{CDCl}_{3}\right) \delta 159.0,149.3,148.0,135.9,128.5,120.2,118.3,117.6$, 108.3, 25.0.<smiles>Cc1ccc2cccc(N)c2n1</smiles>

$2 \mathrm{ab}$

Following the general procedure A, L5 (10 mol\%) was used as ligand, compound $\mathbf{2} \mathbf{a b}{ }^{18}$ was prepared from 8-chloro-2-methylquinoline as dark green solid (124 mg, $78 \%$ yield). ${ }^{1} \mathrm{H}$ NMR $\left(500 \mathrm{MHz}, \mathrm{CDCl}_{3}\right) \delta 7.95(\mathrm{~d}, J=8.4 \mathrm{~Hz}, 1 \mathrm{H}), 7.29-7.21(\mathrm{~m}$, 2H), $7.11(\mathrm{dd}, J=8.1,1.3 \mathrm{~Hz}, 1 \mathrm{H}), 6.90(\mathrm{dd}, J=7.5,1.2 \mathrm{~Hz}, 1 \mathrm{H}), 4.95(\mathrm{br} \mathrm{s}, 2 \mathrm{H})$, $2.71(\mathrm{~s}, 3 \mathrm{H}) ;{ }^{13} \mathrm{C} \mathrm{NMR}\left(100 \mathrm{MHz}, \mathrm{CDCl}_{3}\right) \delta 156.1,143.4,137.9,136.0,126.9,126.3$, $122.1,115.8,110.1,25.2$.<smiles>Nc1cncc2ccccc12</smiles> 
Following the general procedure A, L5 (10 mol\%) was used as ligand, compound $\mathbf{2 a c}^{17}$ was prepared from 4-chloroisoquinoline as yellow solid (115 mg, 80\% yield). ${ }^{1} \mathrm{H}$ NMR $\left(500 \mathrm{MHz}, \mathrm{CDCl}_{3}\right) \delta 8.74(\mathrm{~s}, 1 \mathrm{H}), 8.03(\mathrm{~s}, 1 \mathrm{H})$, 7.95-7.87 (m, 1H), 7.84-7.75 (m, 1H), 7.71-7.62 (m, 1H), 7.62-7.53 (m, 1H), 4.11 (br s, 2H); ${ }^{13} \mathrm{C}$ NMR (100 MHz, $\left.\mathrm{CDCl}_{3}\right) \delta 143.0,137.1,129.0,128.6,128.0,127.7,127.0,126.1,120.1$.<smiles>Nc1ccc2nccnc2[14cH]1</smiles>

Following the general procedure A, compound $\mathbf{2} \mathbf{a d}^{8}$ was prepared from 6-chloroquinoxaline as yellow solid (137 mg, 94\% yield). ${ }^{1} \mathrm{H} \mathrm{NMR}\left(500 \mathrm{MHz}, \mathrm{CDCl}_{3}\right) \delta 8.66$ $(\mathrm{d}, J=2.0 \mathrm{~Hz}, 1 \mathrm{H}), 8.55(\mathrm{~d}, J=1.9 \mathrm{~Hz}, 1 \mathrm{H}), 7.88(\mathrm{~d}, J=8.9 \mathrm{~Hz}, 1 \mathrm{H}), 7.19(\mathrm{dd}, J=$ 9.0, $2.6 \mathrm{~Hz}, 1 \mathrm{H}), 7.14(\mathrm{~d}, J=2.5 \mathrm{~Hz}, 1 \mathrm{H}), 4.23($ br s, $2 \mathrm{H}) ;{ }^{13} \mathrm{C} \mathrm{NMR}(100 \mathrm{MHz}$, $\left.\mathrm{CDCl}_{3}\right) \delta 148.3,145.1,145.0,141.0,138.1,130.5,122.2,107.9$.<smiles>Nc1cc(C(F)(F)F)c[18cH][14cH]1</smiles>

Following the general procedure A, compound $2 \mathbf{a e}^{8}$ was prepared from 3-chloro-5(trifluoromethyl)pyridine as yellow liquid (119 mg, 74\% yield). ${ }^{1} \mathrm{H}$ NMR (400 MHz, $\left.\mathrm{CDCl}_{3}\right) \delta 8.33-8.16(\mathrm{~m}, 2 \mathrm{H}), 7.14(\mathrm{t}, J=2.4 \mathrm{~Hz}, 1 \mathrm{H}), 3.91($ br s, $2 \mathrm{H}) ;{ }^{13} \mathrm{C}$ NMR $(100$ $\left.\mathrm{MHz}, \mathrm{CDCl}_{3}\right) \delta 143.1,139.9,135.3(\mathrm{q}, J=4.4 \mathrm{~Hz}), 127.0(\mathrm{q}, J=32.6 \mathrm{~Hz}), 123.6(\mathrm{q}, J$ $=270.9 \mathrm{~Hz}), 117.5(\mathrm{q}, J=3.7 \mathrm{~Hz})$.<smiles>COc1cccc(N)n1</smiles> 
Following the general procedure A, compound $2 \mathbf{a f}^{8}$ was prepared from 2-chloro-6methoxy-pyridine as light brown liquid (104 mg, 84\% yield). ${ }^{1} \mathrm{H}$ NMR (400 MHz, $\left.\mathrm{CDCl}_{3}\right) \delta 7.33(\mathrm{t}, J=7.8 \mathrm{~Hz}, 1 \mathrm{H}), 6.07(\mathrm{ddd}, J=13.0,7.8,0.6 \mathrm{~Hz}, 2 \mathrm{H}), 4.33$ (br s, 2H), $3.83(\mathrm{~s}, 3 \mathrm{H}) ;{ }^{13} \mathrm{C} \mathrm{NMR}\left(100 \mathrm{MHz}, \mathrm{CDCl}_{3}\right) \delta 163.7,157.4,140.3,99.8,98.2,53.2$.<smiles>Cc1csc2ccc(N)cc12</smiles>

2ag

Following the general procedure A, compound 2ag was prepared from 5-chloro-3methylbenzo[b]thiophene as orange oil (186 mg, 90\% yield). ${ }^{1} \mathrm{H}$ NMR (400 MHz, $\left.\mathrm{CDCl}_{3}\right) \delta 7.60(\mathrm{dd}, J=8.5,0.6 \mathrm{~Hz}, 1 \mathrm{H}), 7.02(\mathrm{~d}, J=1.3 \mathrm{~Hz}, 1 \mathrm{H}), 6.99(\mathrm{~d}, J=0.6 \mathrm{~Hz}$, $1 \mathrm{H}), 6.79(\mathrm{dd}, J=8.5,2.3 \mathrm{~Hz}, 1 \mathrm{H}), 3.70($ br s, $1 \mathrm{H}), 2.36(\mathrm{~d}, J=1.3 \mathrm{~Hz}, 3 \mathrm{H}) ;{ }^{13} \mathrm{C}$ NMR $\left(100 \mathrm{MHz}, \mathrm{CDCl}_{3}\right) \delta 143.6,140.9,131.2,130.5,123.1,122.2,114.5,106.6$, 13.8; ESI-HRMS m/z calcd. for $\mathrm{C}_{9} \mathrm{H}_{10} \mathrm{NS}(\mathrm{M}+\mathrm{H})^{+}:$164.0528, found: 164.0532 .<smiles>Nc1ccc2c(ccn2Br)c1</smiles>

Following the general procedure A, compound $\mathbf{2} \mathbf{a h}^{19}$ was prepared from 5-chloro-1benzyl-1H-indole as orange red oil (194 mg, 87\% yield). ${ }^{1} \mathrm{H} \mathrm{NMR}\left(400 \mathrm{MHz}, \mathrm{CDCl}_{3}\right)$ $\delta 7.32-7.26(\mathrm{~m}, 3 \mathrm{H}), 7.12-7.04(\mathrm{~m}, 4 \mathrm{H}), 6.95(\mathrm{dd}, J=2.2,0.6 \mathrm{~Hz}, 1 \mathrm{H}), 6.63(\mathrm{dd}, J=$ 8.6, $2.2 \mathrm{~Hz}, 1 \mathrm{H}), 6.37(\mathrm{dd}, J=3.1,0.9 \mathrm{~Hz}, 1 \mathrm{H}), 5.26(\mathrm{~s}, 2 \mathrm{H}), 3.18(\mathrm{~s}, 2 \mathrm{H}) ;{ }^{13} \mathrm{C} \mathrm{NMR}$ $\left(125 \mathrm{MHz}, \mathrm{CDCl}_{3}\right) \delta 139.5,137.9,131.5,129.8,128.8,127.6,126.8,112.8,110.4$, $106.0,100.5,50.3$<smiles>Nc1ccc2c(c1)c1ccccc1n2Cc1ccccc1</smiles> 
Following the general procedure A, compound $\mathbf{2} \mathbf{a i}^{20}$ was prepared from 9-benzyl-3chloro-9H-carbazole as grey solid (239 mg, 88\% yield). ${ }^{1} \mathrm{H}$ NMR (400 $\mathrm{MHz}, d_{6^{-}}$ DMSO) $\delta 7.96(\mathrm{~d}, J=7.7 \mathrm{~Hz}, 1 \mathrm{H}), 7.49(\mathrm{~d}, J=8.2 \mathrm{~Hz}, 1 \mathrm{H}), 7.36-7.28(\mathrm{~m}, 3 \mathrm{H}), 7.28-$ $7.16(\mathrm{~m}, 3 \mathrm{H}), 7.16-7.11(\mathrm{~m}, 2 \mathrm{H}), 7.08(\mathrm{t}, J=7.4 \mathrm{~Hz}, 1 \mathrm{H}), 6.79(\mathrm{dd}, J=8.7,2.1 \mathrm{~Hz}$, 1H), $5.52(\mathrm{~s}, 2 \mathrm{H}), 4.76(\mathrm{~s}, 2 \mathrm{H}) ;{ }^{13} \mathrm{C} \mathrm{NMR}\left(100 \mathrm{MHz}, d_{6}\right.$-DMSO) $\delta 141.8,140.4,138.2$, $133.3,128.4,127.1,126.7,125.2,122.9,121.9,120.0,117.9,115.1,109.8,109.1$, $104.0,45.5$.<smiles>Nc1ccn2ccnc2c1</smiles>

2aj

Following the general procedure A, L5 (10 mol\%) was used as ligand, compound 2aj was prepared from 7-chloroimidazo[1,2a]pyridine as pale grey solid (97 mg, 73\% yield). ${ }^{1} \mathrm{H}$ NMR (400 MHz, d6-DMSO) $\delta 8.13(\mathrm{~d}, J=8.0 \mathrm{~Hz}, 1 \mathrm{H}), 7.50(\mathrm{~s}, 1 \mathrm{H}), 7.18$ $(\mathrm{d}, J=1.4 \mathrm{~Hz}, 1 \mathrm{H}), 6.44-6.31(\mathrm{~m}, 2 \mathrm{H}), 5.64(\mathrm{br} \mathrm{s}, 2 \mathrm{H}) ;{ }^{13} \mathrm{C} \mathrm{NMR}(100 \mathrm{MHz}, d 6-$ DMSO) $\delta 147.1,146.4,131.3,126.8,110.4,106.5,92.3$; ESI-HRMS $m / z$ calcd. for $\mathrm{C}_{7} \mathrm{H}_{8} \mathrm{~N}_{3}(\mathrm{M}+\mathrm{H})^{+}$134.0718, found: 134.0719 .<smiles>Nc1cnn([Ga])c1</smiles>

Following the general procedure B (Note: $2 \mathrm{~mL}$ DMSO was used because $1.0 \mathrm{M}$ was too concentrated to stir smoothly), compound $\mathbf{2} \mathbf{a k}^{8}$ was prepared from 4-chloro-1(triphenylmethyl)-1H-pyrazole as orange solid (117 mg, 72\% yield). ${ }^{1} \mathrm{H}$ NMR (400 $\left.\mathrm{MHz}, \mathrm{CDCl}_{3}\right) \delta$ 7.36-7.27 (m, 10H), 7.20-7.12 (m, 6H), $6.96(\mathrm{~d}, \mathrm{~J}=1.0 \mathrm{~Hz}, 1 \mathrm{H}), 2.17$ 
(br s, $2 \mathrm{H}) ;{ }^{13} \mathrm{C} \mathrm{NMR}\left(100 \mathrm{MHz}, \mathrm{CDCl}_{3}\right) \delta 143.4,132.0,130.2,127.7,127.7,127.6$,

121.6, 78.3.<smiles>Nc1nn(Cc2ccccc2)c2ccccc12</smiles>

Following the general procedure B, compound $\mathbf{2} \mathbf{a l}^{8}$ was prepared from 1-benzyl-3chloro-1H-indazole as brown solid (169 mg, 76\% yield). ${ }^{1} \mathrm{H}$ NMR (400 MHz, $\mathrm{CDCl}_{3}$ ) $\delta 7.55(\mathrm{dt}, J=8.1,0.9 \mathrm{~Hz}, 1 \mathrm{H}), 7.35-7.26(\mathrm{~m}, 3 \mathrm{H}), 7.26-7.15(\mathrm{~m}, 4 \mathrm{H}), 7.03(\mathrm{ddd}, J=$ 7.9, 6.9, $0.8 \mathrm{~Hz}, 1 \mathrm{H}), 5.37$ (s, 2H), 4.08 (br s, $2 \mathrm{H}) ;{ }^{13} \mathrm{C} \mathrm{NMR}\left(100 \mathrm{MHz}, \mathrm{CDCl}_{3}\right) \delta$ 147.6, 141.3, 137.5, 128.6, 127.4, 127.0, 127.0, 119.7, 118.6, 114.9, 108.9, 52.0.

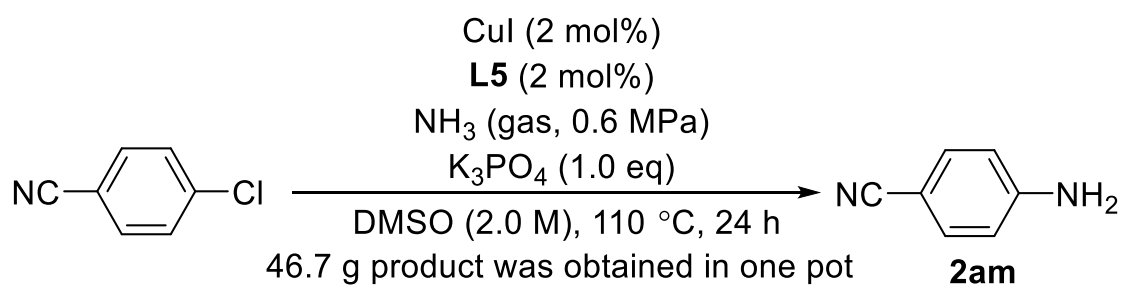

4-Chlorobenzonitrile (67.13 g, $488 \mathrm{mmol}), \mathrm{CuI}$ (1.87 g, $9.8 \mathrm{mmol})$, L5 (4.12 g, 9.8 mmol), $\mathrm{K}_{3} \mathrm{PO}_{4}(103.6 \mathrm{~g}, 488 \mathrm{mmol})$ and $245 \mathrm{~mL}$ DMSO were mixed in a $1 \mathrm{~L}$ lowpressure reaction kettle and then the reaction kettle was charged with $\mathrm{NH}_{3}$ gas. (Note: When charging $\mathrm{NH}_{3}$ gas to reaction kettle, longstanding stirring was needed at the same time to ensure enough $\mathrm{NH}_{3}$ was dissolved in DMSO.) Under mechanical stirring, the temperature inside the reaction kettle was raised to $110{ }^{\circ} \mathrm{C}$ and kept for $24 \mathrm{~h}$. After the reaction kettle was cooled to room temperature, the slurry was filtered through celite, washed with ethyl acetate $(\sim 400 \mathrm{~mL})$, and transferred into a separating funnel. After brine ( $\sim 300 \mathrm{~mL})$ was added, organic phase was separated and the aqueous phase was extracted with ethyl acetate twice. The combined organic phase was dried over 
$\mathrm{Na}_{2} \mathrm{SO}_{4}$ and concentrated in vacuo. The residue was purified by silica gel flash chromatography to afford 4-aminobenzonitrile $\left(\mathbf{2 a m}^{8}\right)$ as pale yellow solid $(46.7 \mathrm{~g}$, $81 \%$ yield). ${ }^{1} \mathrm{H}$ NMR $\left(500 \mathrm{MHz}, \mathrm{CDCl}_{3}\right) \delta 7.37(\mathrm{~d}, J=8.7 \mathrm{~Hz}, 1 \mathrm{H}), 6.63(\mathrm{~d}, J=8.6$ $\mathrm{Hz}, 2 \mathrm{H}), 4.25$ (br s, $2 \mathrm{H}) ;{ }^{13} \mathrm{C} \mathrm{NMR}\left(125 \mathrm{MHz}, \mathrm{CDCl}_{3}\right) \delta 150.7,133.8,120.3,114.5$, 99.8.<smiles>C=CCCc1ccccc1N</smiles>

Following the general procedure B, compound $2 \mathbf{2 a n}^{13}$ was prepared from 1-(but-3enyl)-2-chlorobenzene as yellow liquid (76 mg, 53\% yield). ${ }^{1} \mathrm{H}$ NMR (400 MHz, $\left.\mathrm{CDCl}_{3}\right) \delta 7.11-7.01(\mathrm{~m}, 2 \mathrm{H}), 6.75(\mathrm{td}, J=7.5,1.3 \mathrm{~Hz}, 1 \mathrm{H}), 6.69(\mathrm{~d}, J=7.3 \mathrm{~Hz}, 1 \mathrm{H})$, $5.92(\mathrm{ddt}, J=16.8,10.2,6.5 \mathrm{~Hz}, 1 \mathrm{H}), 5.10(\mathrm{dq}, J=17.1,1.6 \mathrm{~Hz}, 1 \mathrm{H}), 5.05-4.99(\mathrm{~m}$, 1H), 3.35 (br s, 2H), 2.67-2.52 (m, 2H), 2.45-2.32 (m, 2H); ${ }^{13} \mathrm{C}$ NMR (100 MHz, $\left.\mathrm{CDCl}_{3}\right) \delta 144.2,138.2,129.5,127.1,126.0,118.9,115.7,115.2,32.9,30.8$.

\section{References:}

1. Stylianides, N.; Danopoulos, A. A.; Pugh, D.; Hancock, F.; Zanotti-Gerosa, A. Organometallics 2007, 26, 5627.

2. Ibrahim, Y. A.; Al-Awadi, N. A.; Al-Azemi, T. F.; John, E. RSC Advances 2014, 4, 38869 .

3. Walls, L. P. J. Chem. Soc. 1934, 104.

4. Lubriks, D.; Sokolovs, I.; Suna, E. Org. Lett. 2011, 13, 4324.

5. Hadei, N.; Kantchev, E. A. B.; O'Brie, C. J.; Organ, M. G. J. Org. Chem. 2005, $70,8503$.

6. Yang, X.; Zhang, Y.; Ma, D. Adv. Synth. Catal. 2012, 354, 2443.

7. Kim, J.; Chang, S. Chem. Commun. 2008, 3052. 
8. Cheung, C. W.; Surry, D. S.; Buchwald, S. L. Org. Lett. 2013, 15, 3734.

9. Green, R. A.; Hartwig, J. F. Angew. Chem. Int. Ed. 2015, 54, 3768.

10. Voth, S.; Hollett, J. W.; McCubbin, J. A. J. Org. Chem. 2015, 80, 2545.

11. Emokpae, T. A.; Dosunmu, I. M.; Hirst, J. J. Chem. Soc., Perkin Trans. 2 1977, 14.

12. Xu, H.-J.; Liang, Y.-F.; Cai, Z.-Y.; Qi, H.-X.; Yang, C.-Y.; Feng, Y.-S. J. Org. Chem. 2011, 76, 2296.

13. Green, R. A.; Hartwig, J. F. Org. Lett. 2014, 16, 4388.

14. Fairhurst, R. A.; Janus, D.; Lawrence, A. Org. Lett. 2005, 7, 4697.

15. Sharpless, D.; Spengler, G.; Molnár, J.; Antal, Z.; Molnár, A.; Kiss, J. T.; Szabó, J. A.; Hilgeroth, A.; Gallo, S.; Mahamoud, A.; Barbe, J. Eur. J. Med. Chem. 2005, $40,195$.

16. Sharma, P.; Liu, R.-S. Chem. Eur. J. 2015, 21, 4590.

17. Borzenko, A.; Rotta-Loria, N. L.; MacQueen, P. M.; Lavoie, C. M.; McDonald, R.; Stradiotto, M. Angew. Chem. Int. Ed. 2015, 54, 3773.

18. Yu, J.; Wang, Y.; Zhang, P.; Wu, J. Synlett 2013, 24, 1448.

19. Dey, R.; Mukherjee, N.; Ahammed, S.; Ranu, B. C. Chem. Commun. 2012, 48, 7982.

20. Huo, Z.; Li, Z.; Wang, T.; Zeng, H. Tetrahedron 2013, 69, 8964. 


\section{Copies of ${ }^{1} \mathrm{H}$ and ${ }^{13} \mathrm{C}$ Spectrum}
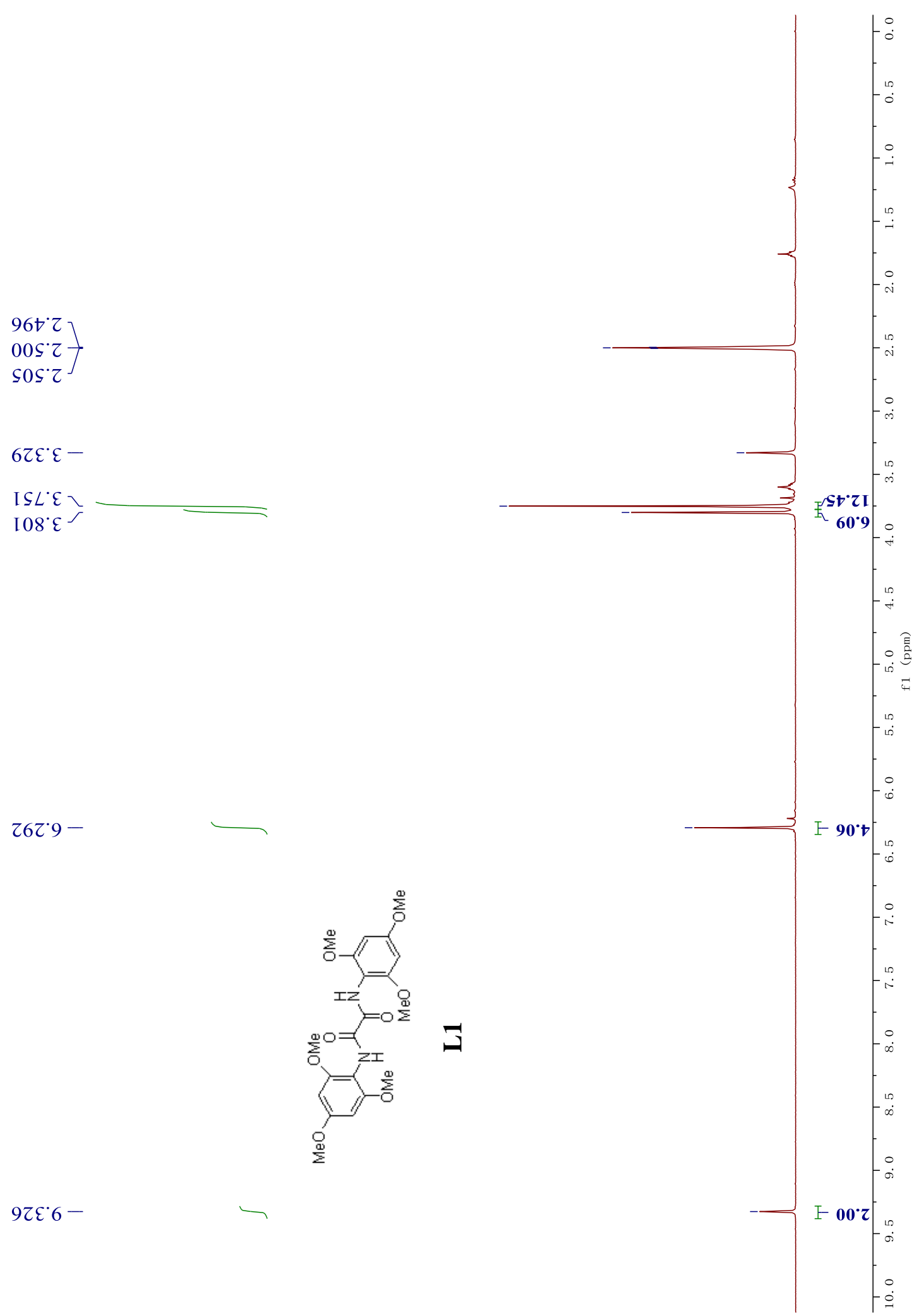

$97 \varepsilon \cdot 6-$ 


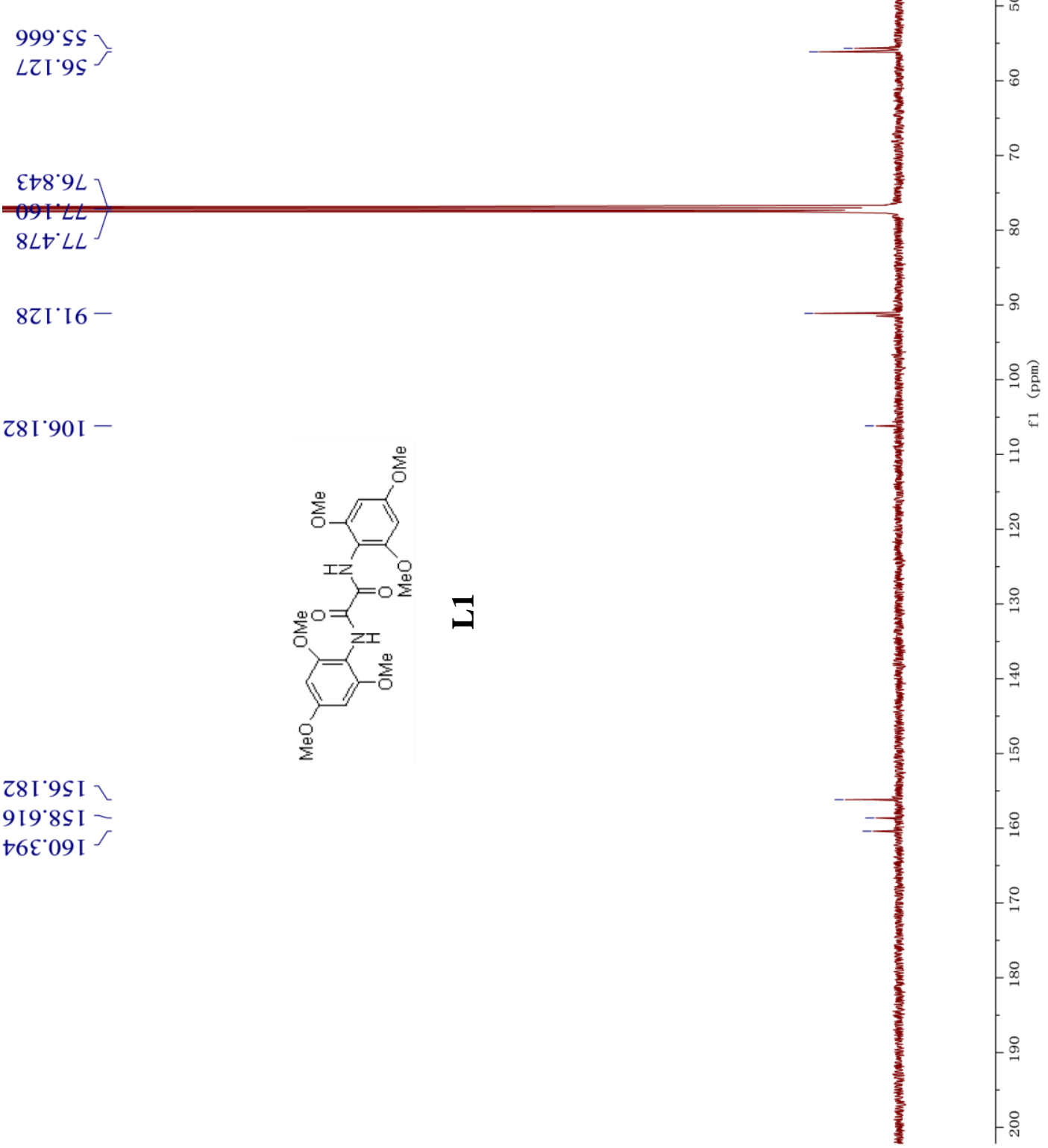




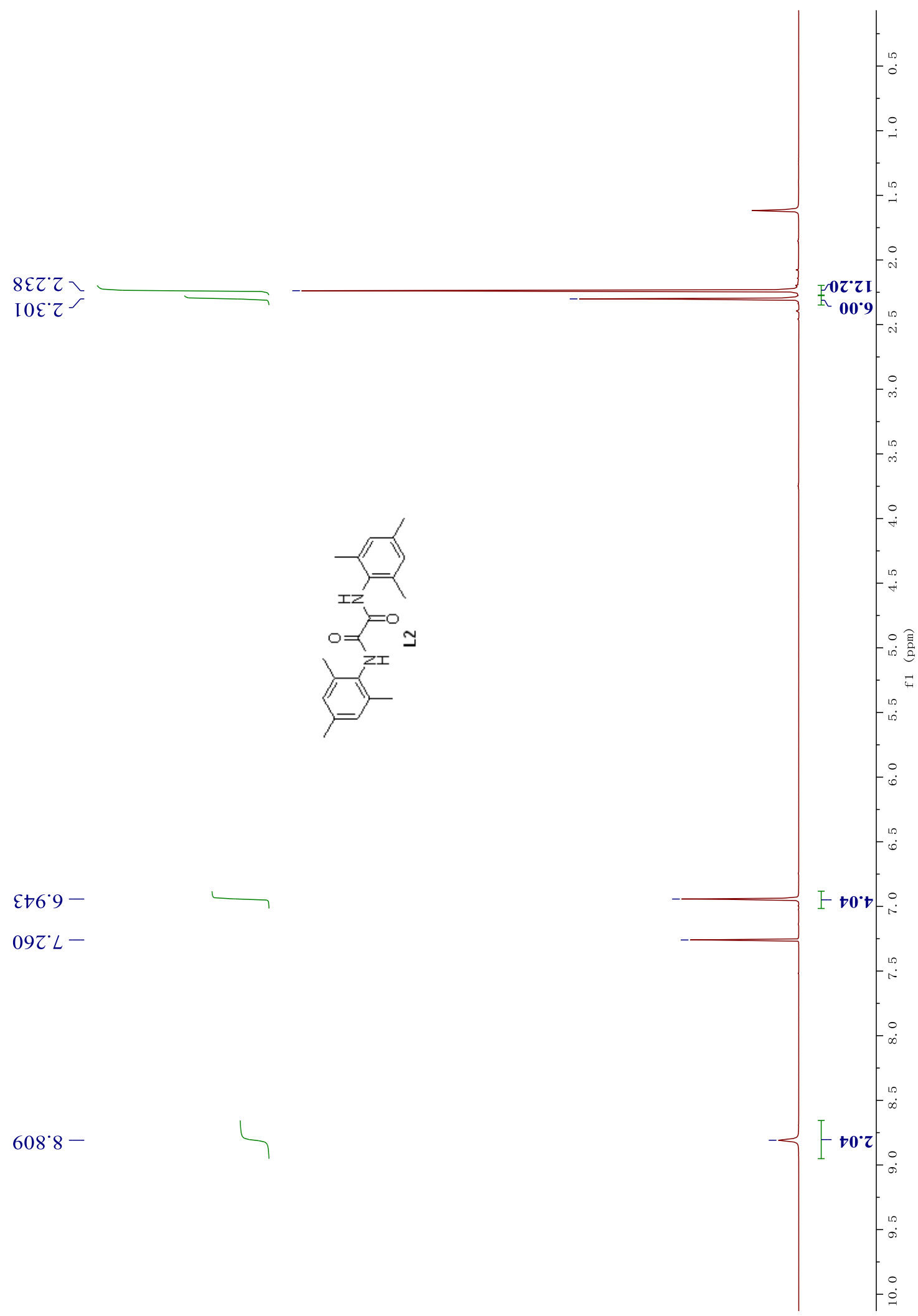


I $\angle 7^{\circ} 8 \mathrm{I}-$ E60'I乙-

$\varepsilon \sqcup 8^{\circ} 9 L$

$09 I^{\circ} L L \frac{1}{J}$

$8 \angle \nabla^{\circ} L L$

LIZ'6ZI

L6L 6ZI 5

SI $8^{\circ} \dagger \varepsilon$ I -

$9 \triangleright L^{\circ} L \mathcal{L} \mathrm{I}^{\digamma}$
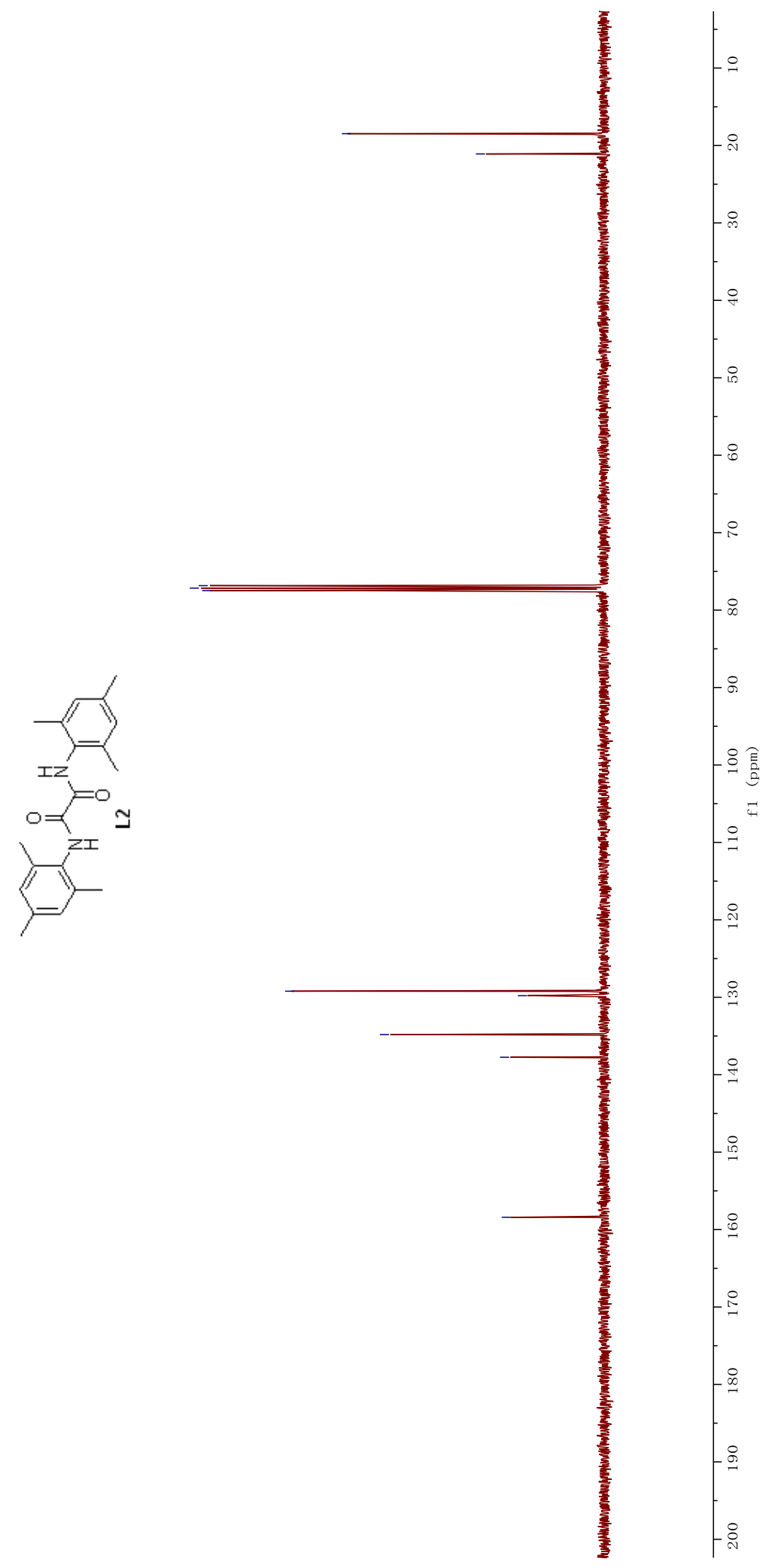

IIt 8 S I - 
I90 Z

$\varsigma 6 t^{\circ} \mathrm{Z}$

$66 t^{\circ} \tau$

$\downarrow 0 \varsigma^{\prime} \tau$

$60 \varsigma^{\circ} \tau$

$8 \mathcal{E} \mathcal{E}^{\cdot} \mathcal{E}-$

$967^{\circ} 9-$

$\varepsilon \varsigma Z^{\circ} 6-$

$\angle S 6^{\circ} 6^{-}$

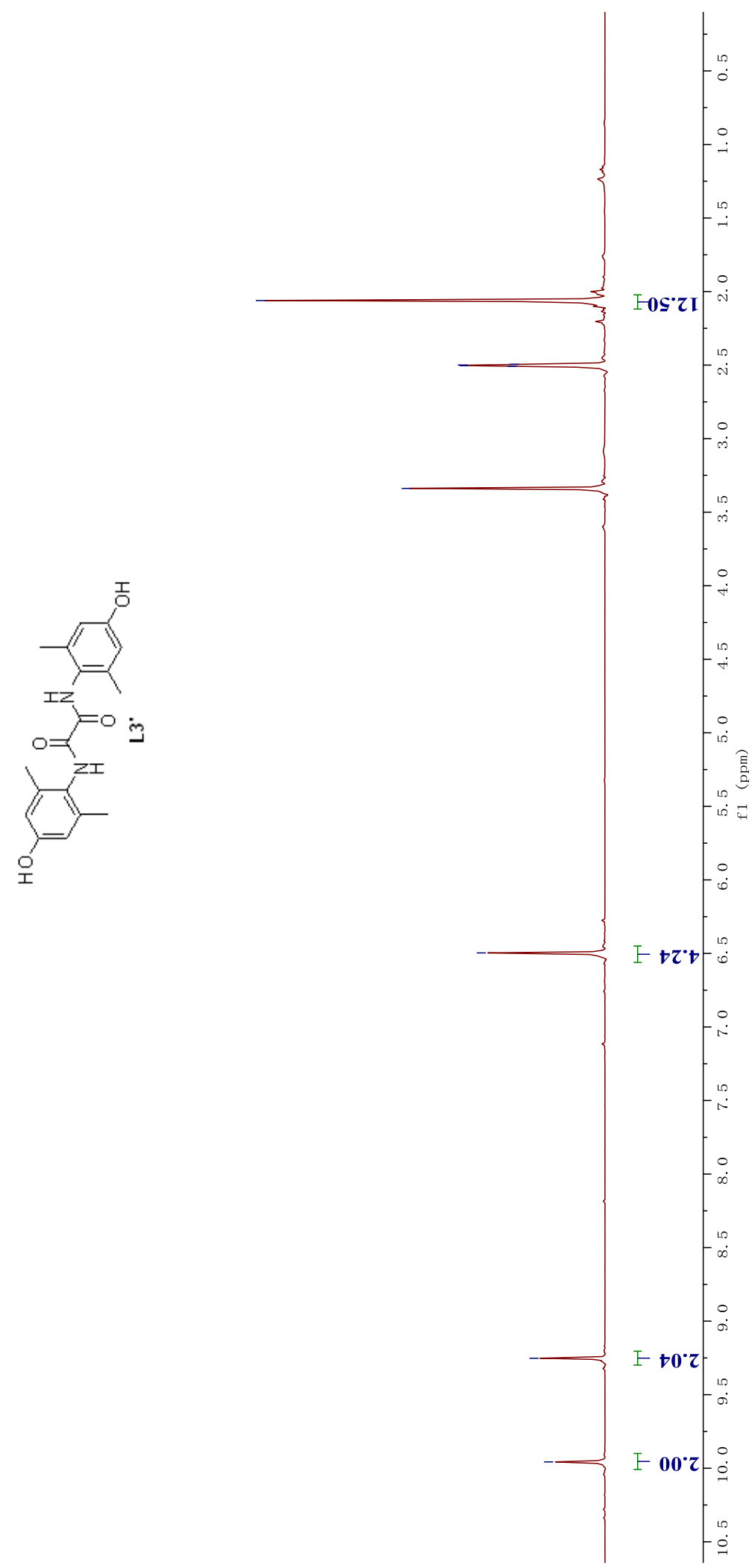




$$
\begin{aligned}
& 9 \mathrm{SI}^{\circ} 8 \mathrm{I} \\
& \varsigma 68^{\circ} 8 \mathcal{\varepsilon} \\
& \varepsilon 0 I^{\circ} 6 \varepsilon \\
& \text { ZI } \varepsilon^{\circ} 6 \varepsilon \\
& \text { IZS. } 6 \varepsilon \\
& 0 \varepsilon L \cdot 6 \varepsilon \\
& \angle \varepsilon 6^{\circ} 6 \varepsilon \\
& \text { st [ }{ }^{\circ} 0 t
\end{aligned}
$$

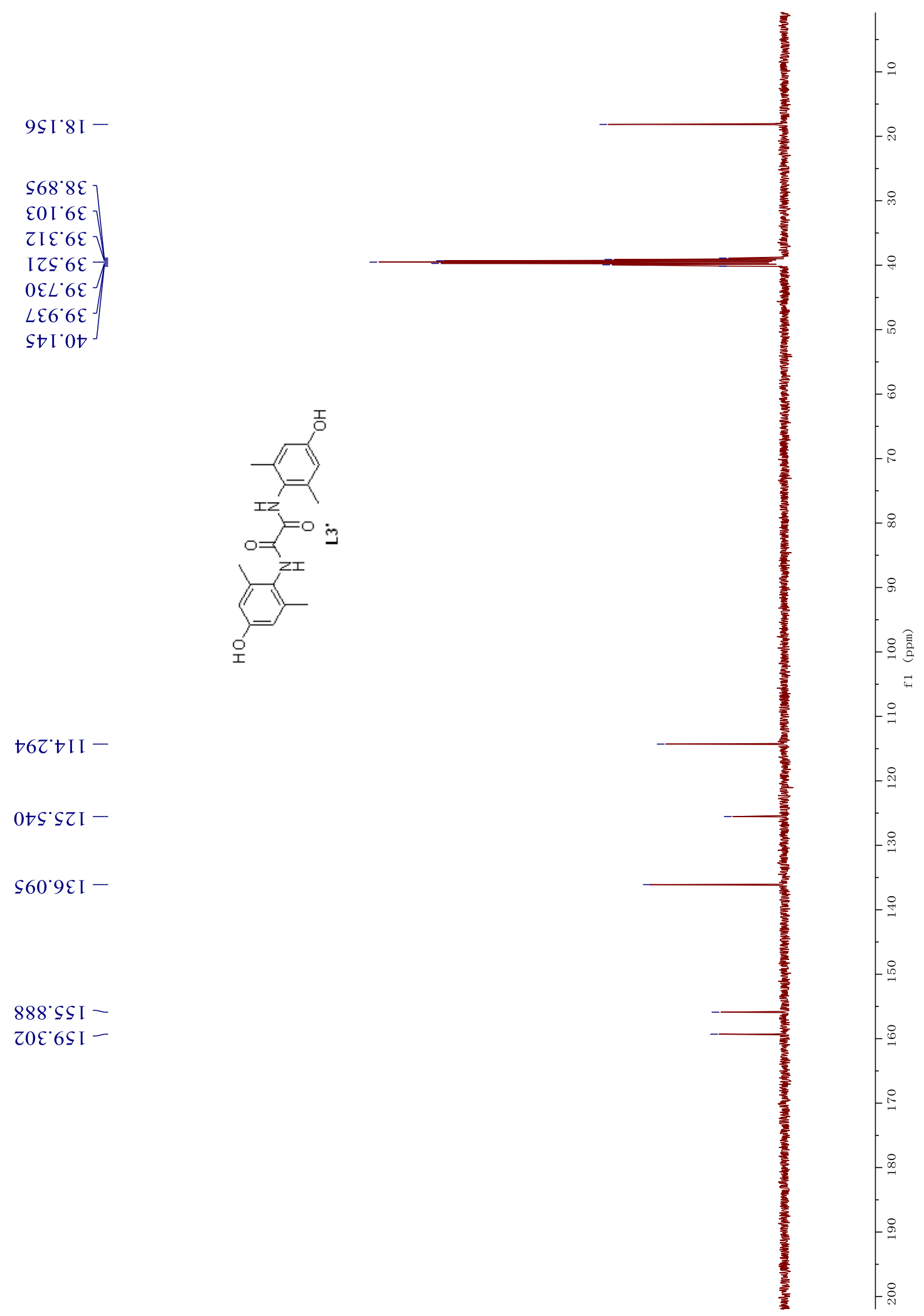

$888^{\circ}$ SS I -

Z0E.6S I - 


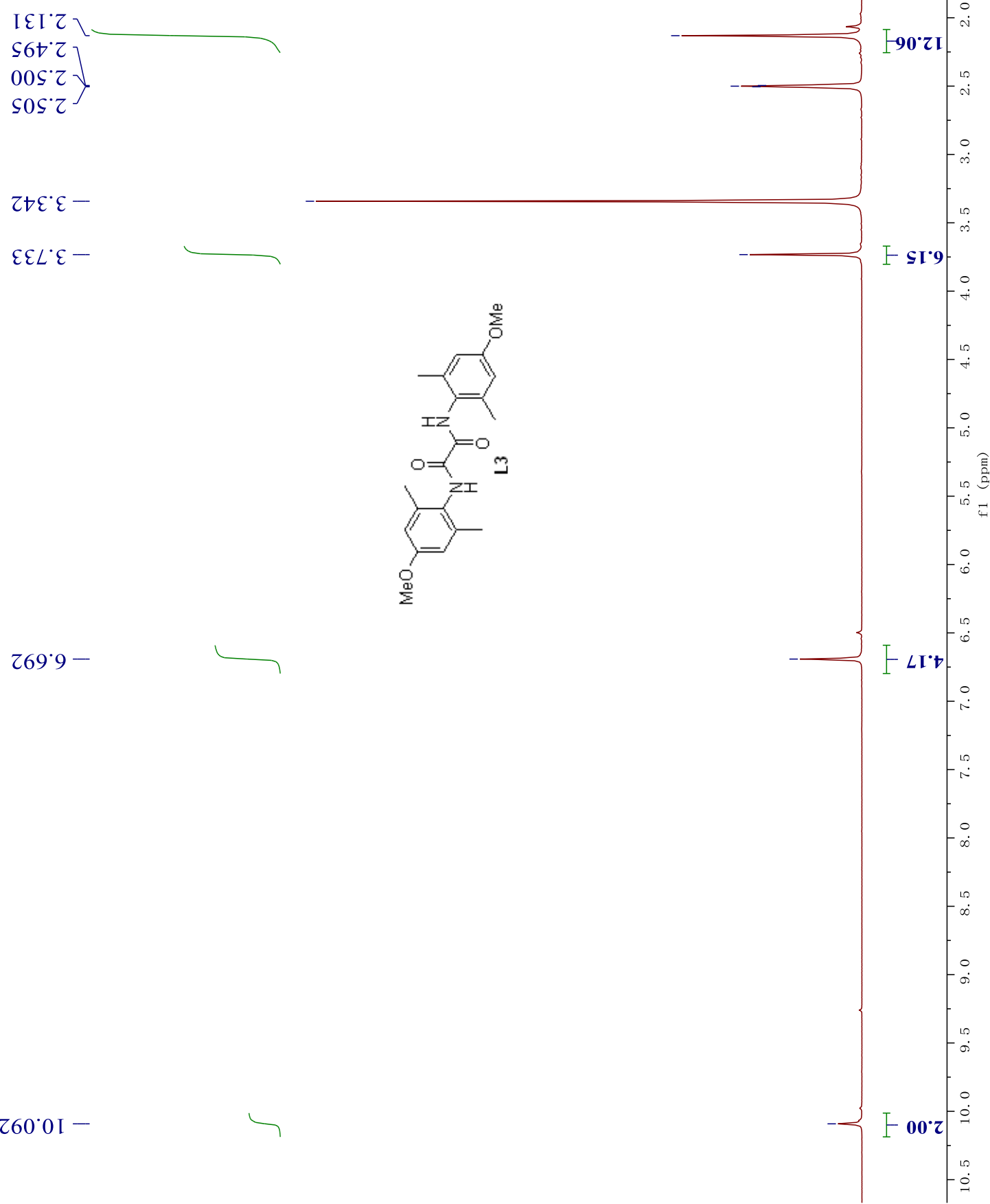




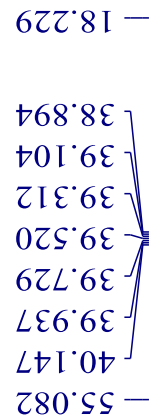

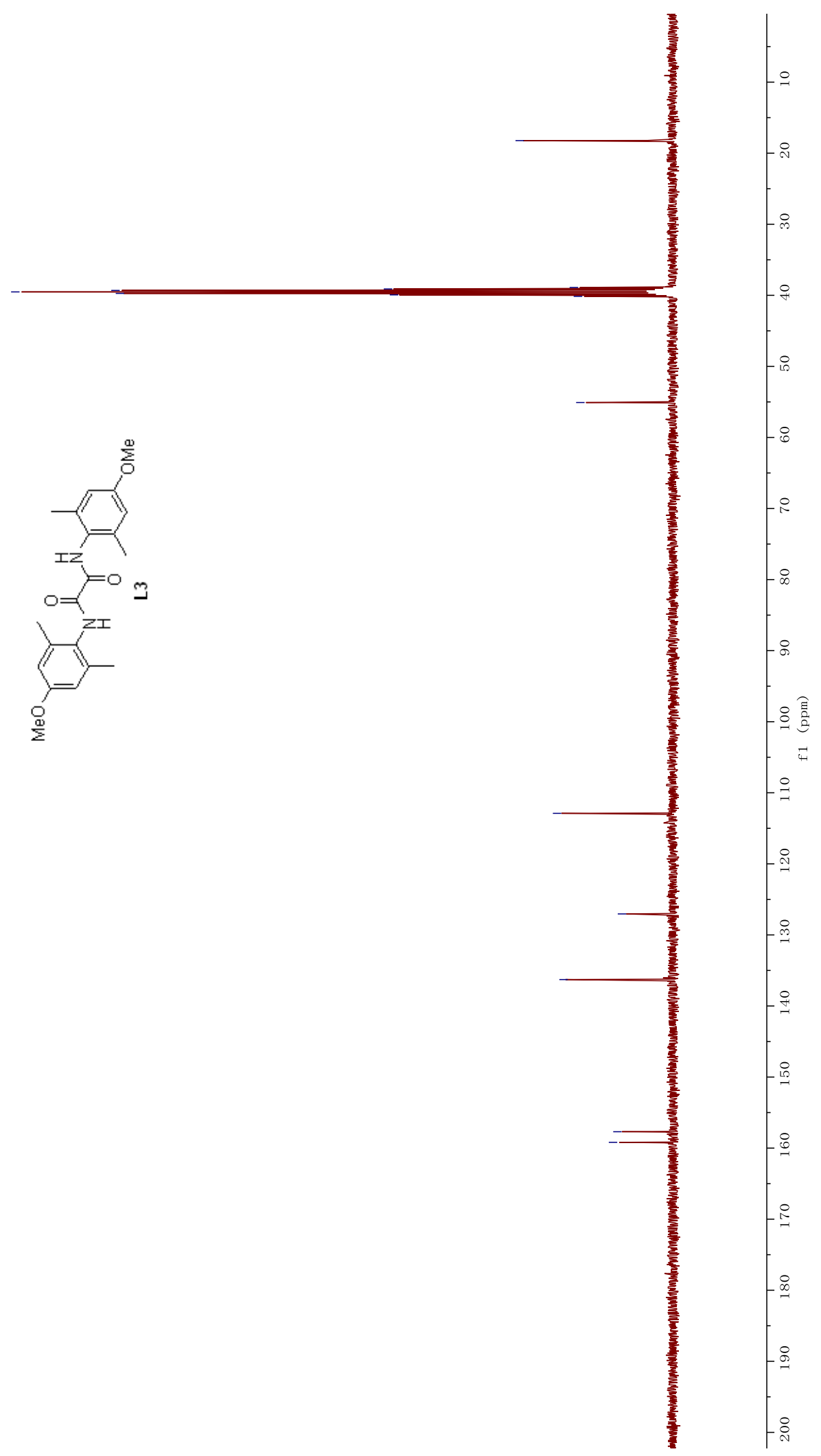

S68`ZII -

$\angle t 00^{\circ} \angle Z I-$

$\triangleright 8 \tau^{\circ} 9 \varepsilon \mathrm{I}-$

$\angle 69^{\circ} \angle S I 乙$

$98 \mathrm{I}^{\circ} 6 \mathrm{~S}^{-}$ 


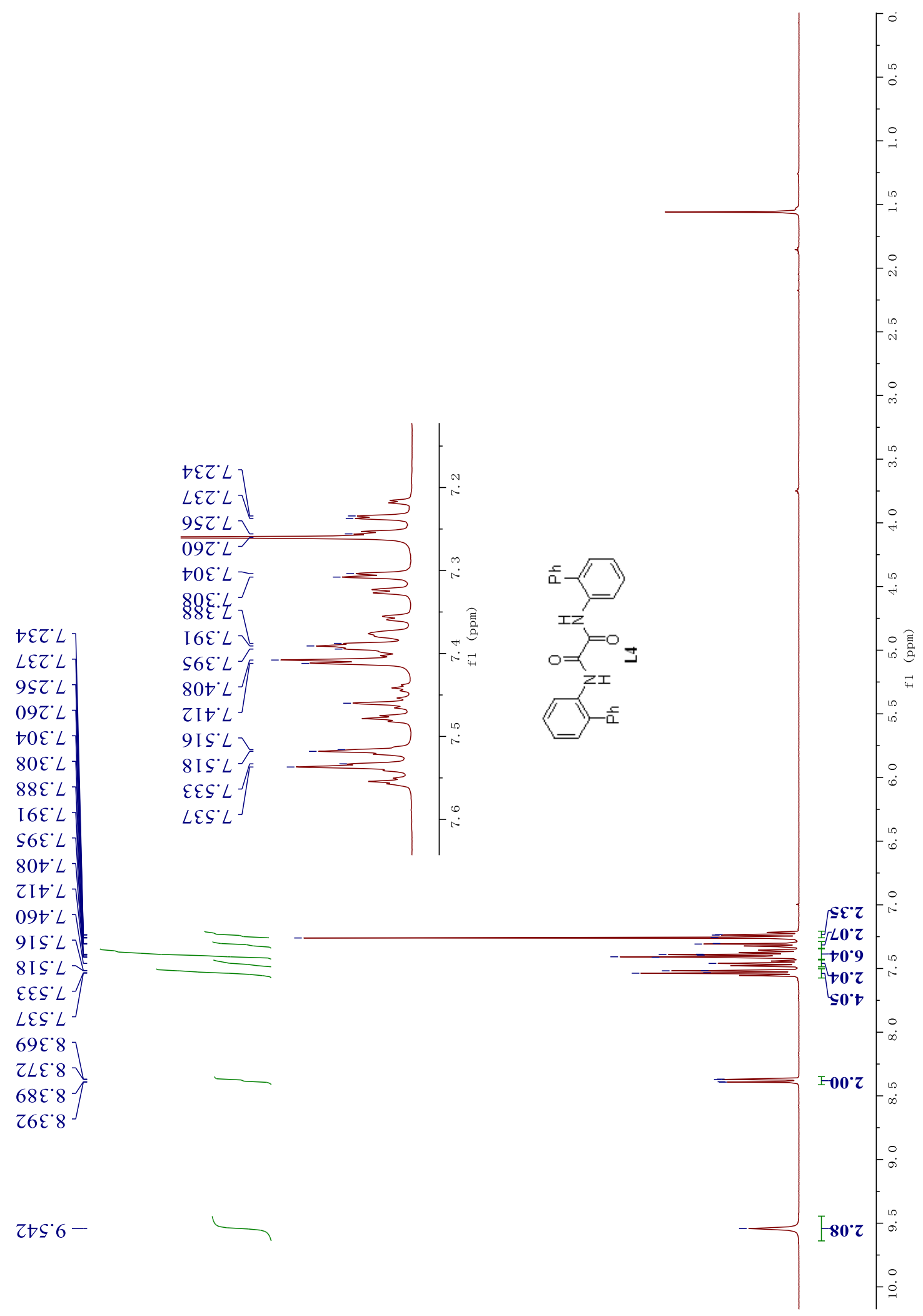




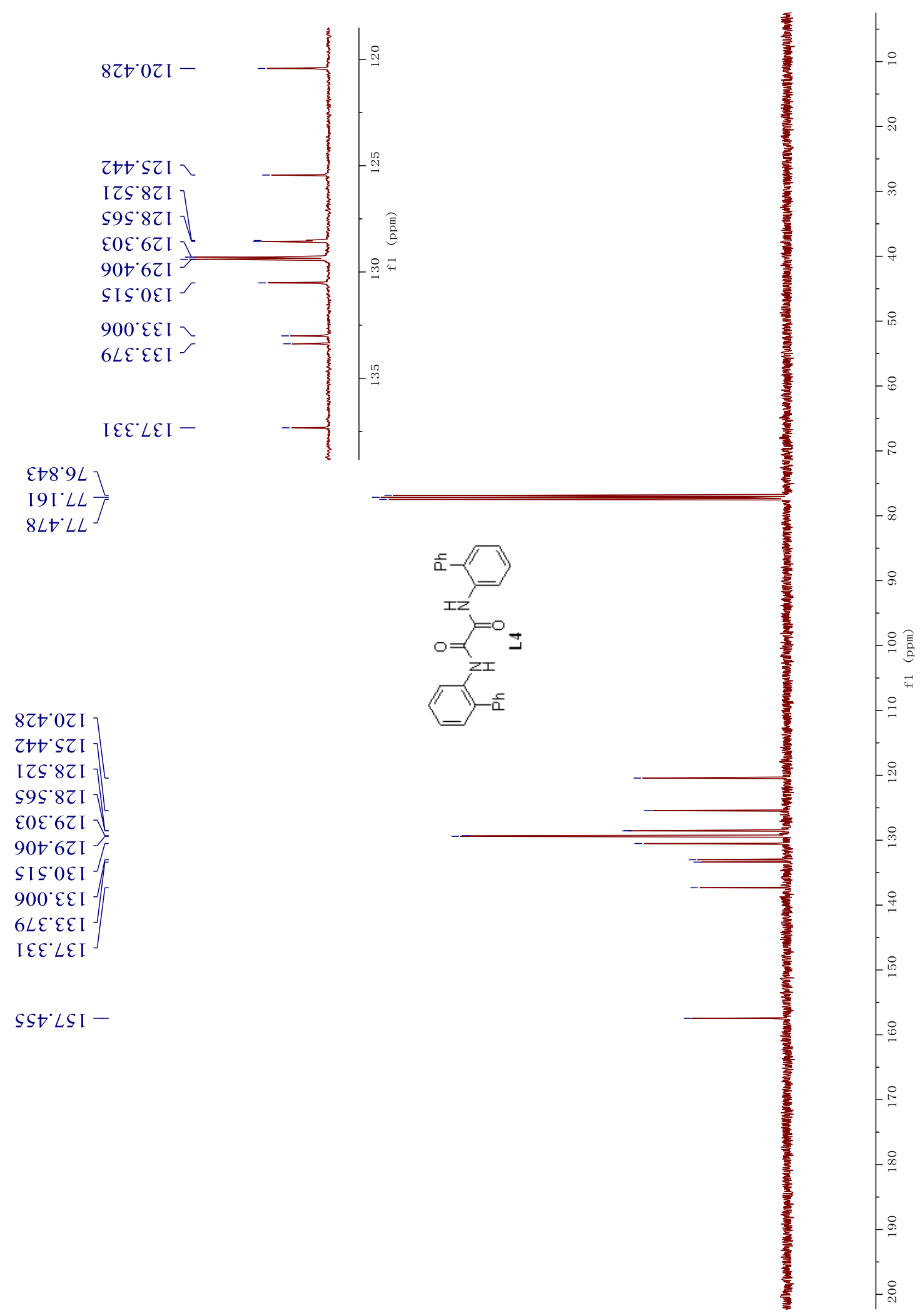


I9ع $て-$

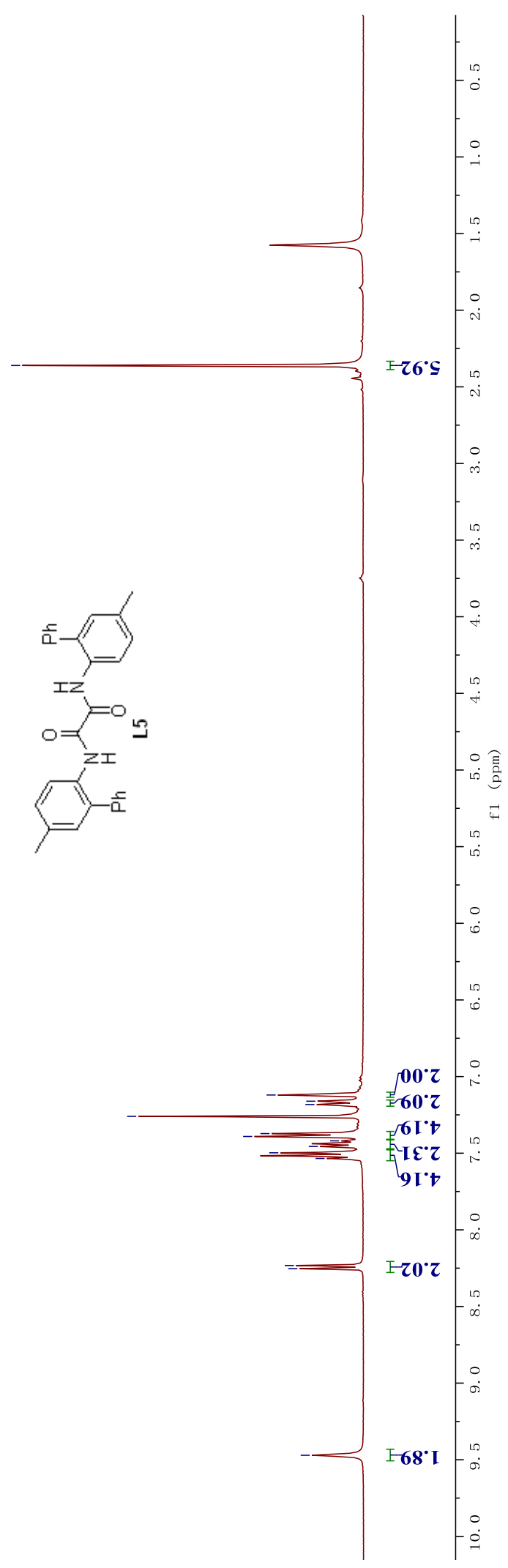




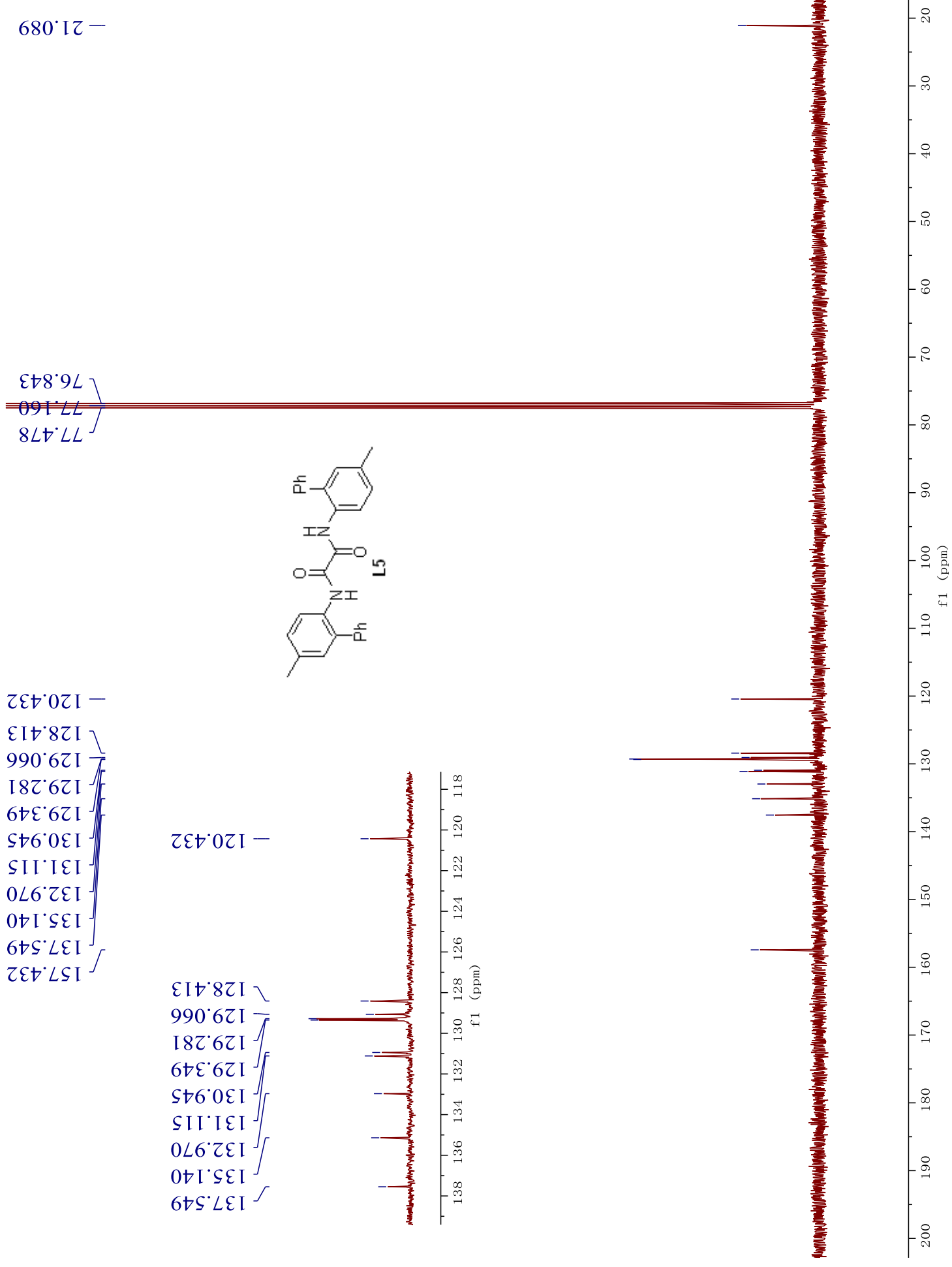




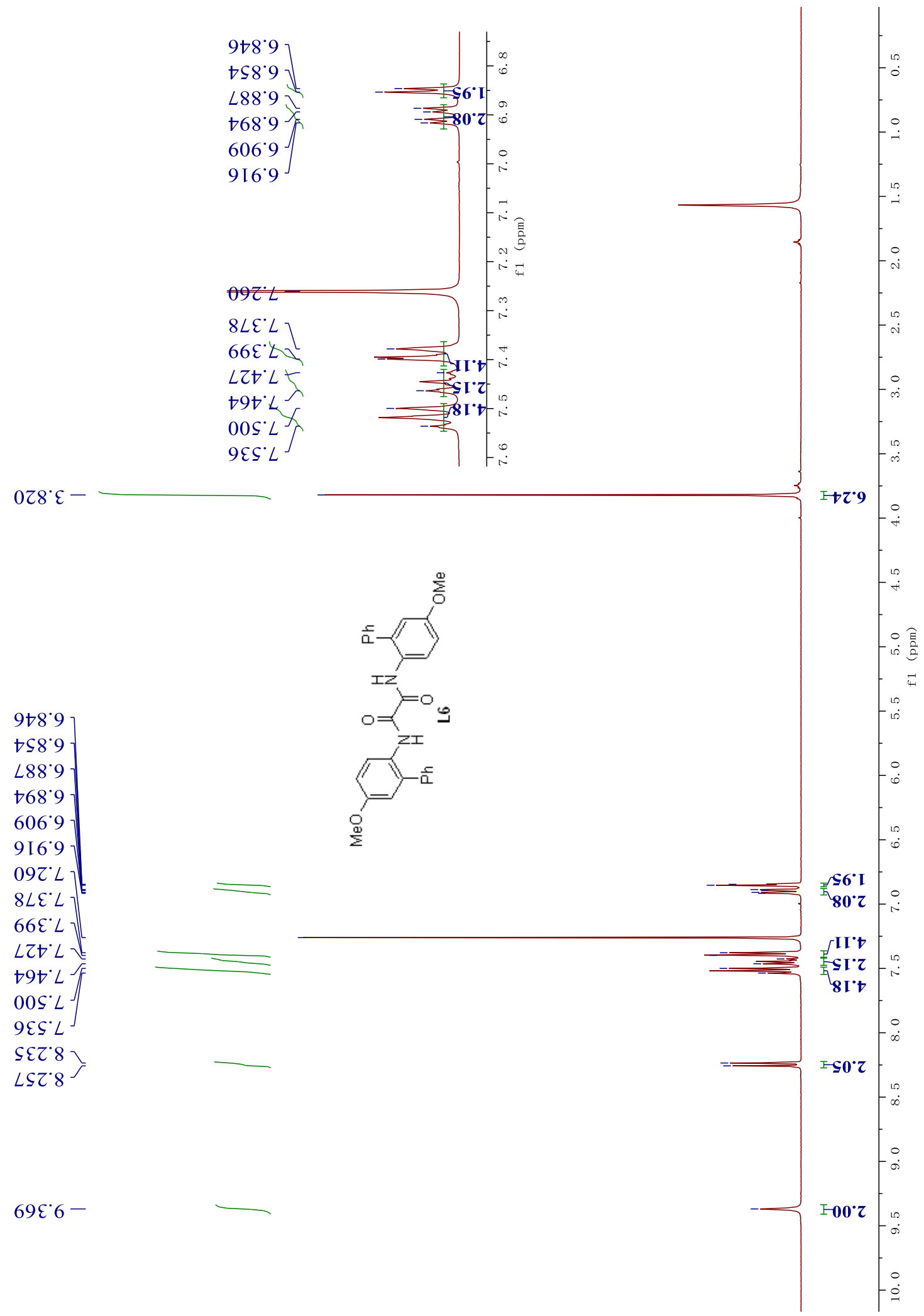




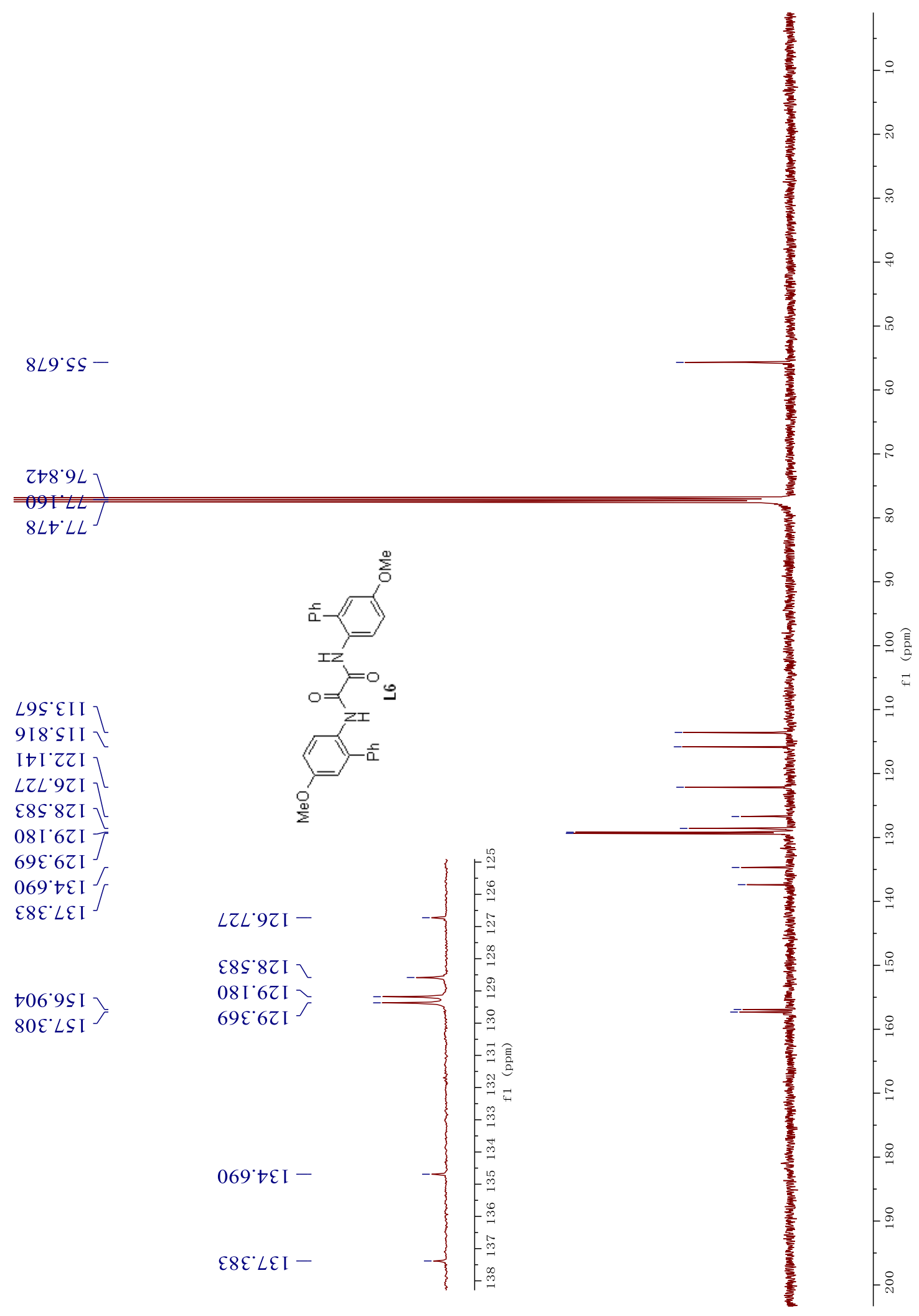


$6 S^{\circ} I^{-}$

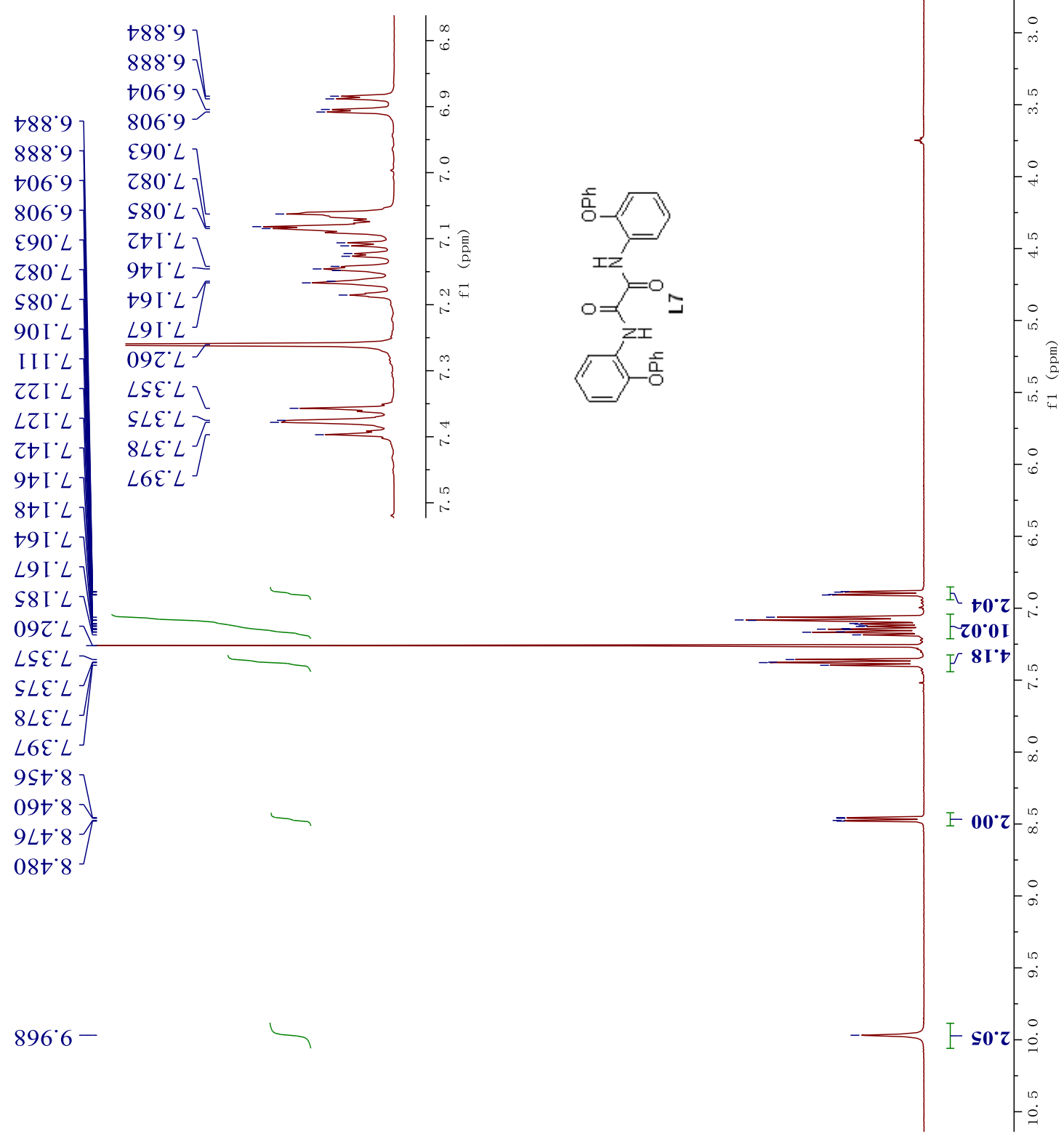




$$
\left.\begin{array}{l}
\varepsilon+8^{\circ} 9 L \\
09 I^{\circ} L L \\
087^{\circ} L L
\end{array}\right]
$$

ZEL L L I

$8 \varepsilon$ I $^{\circ} 6$ I I S09.0ZI 8L8 $\varepsilon Z$ I $\varepsilon 0 \varepsilon^{\circ} \dagger$ I I $0 Z \varsigma^{\circ} \varsigma \mathrm{I}$ $660^{\circ} 8 Z$ I S60 $00^{\circ}$ I

$6 \varepsilon 8^{\circ} 9$ t I 88 I. $^{\circ} 9$ I $ح$ $0 \angle D^{\circ} L S I^{\prime}$
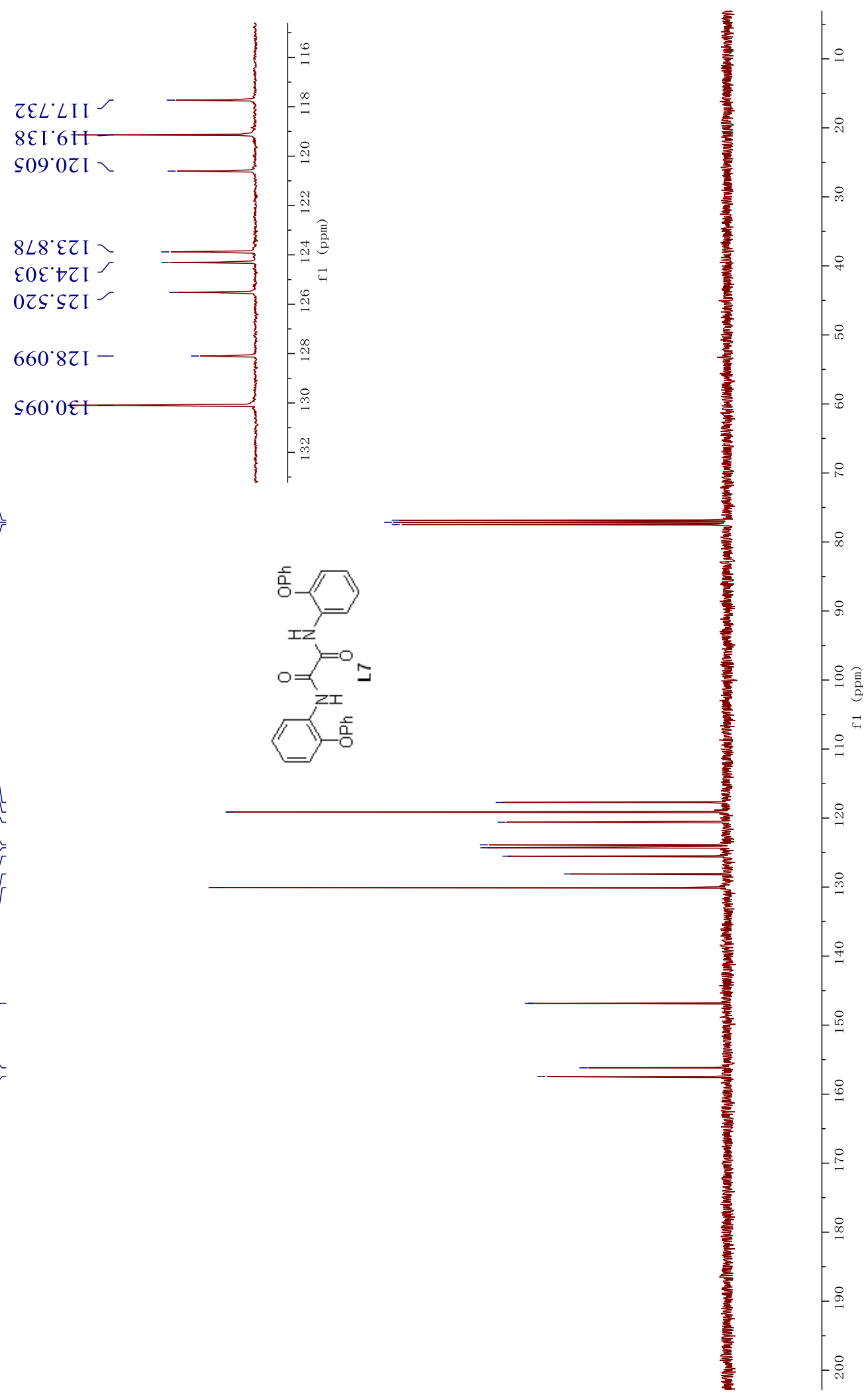


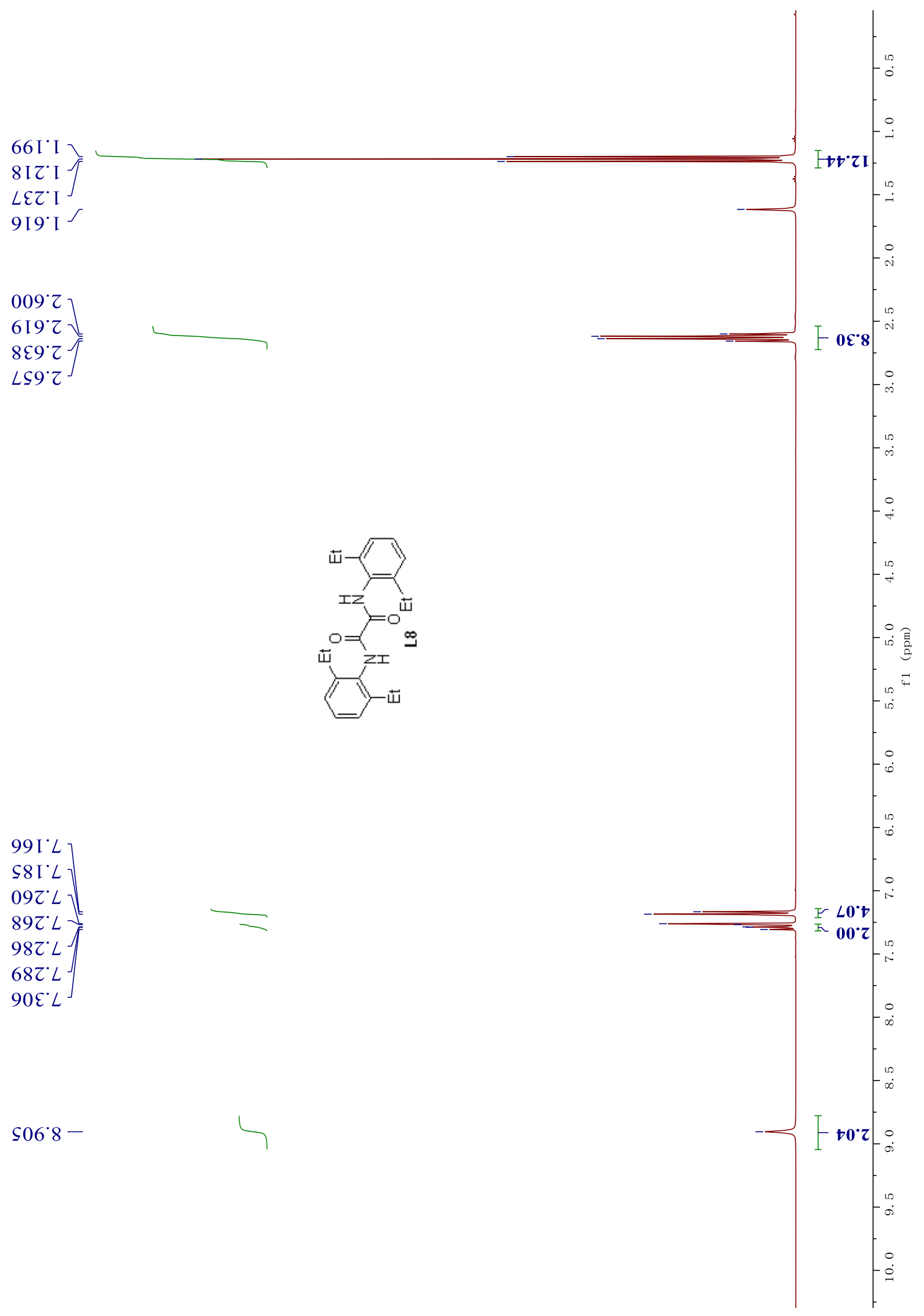



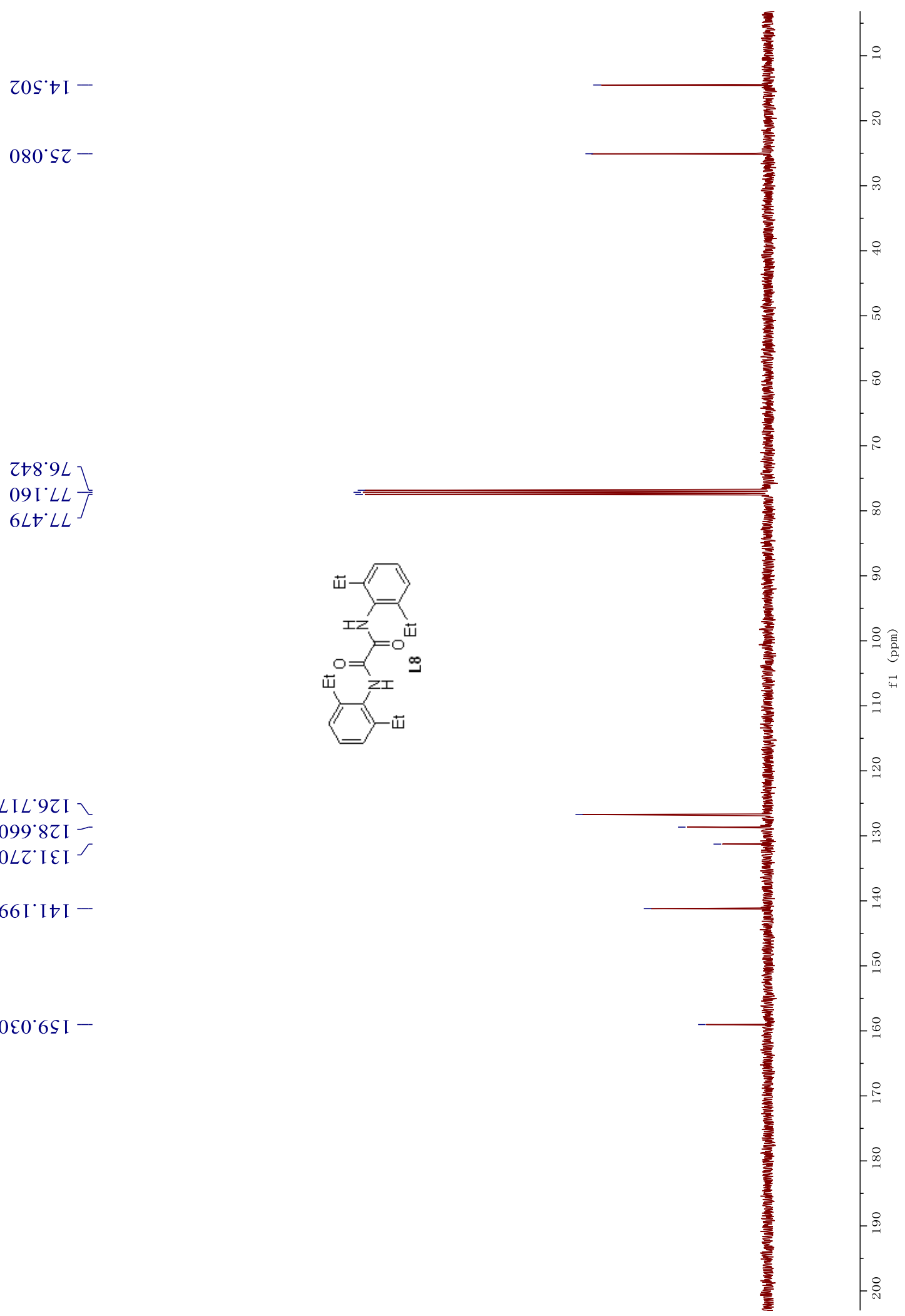


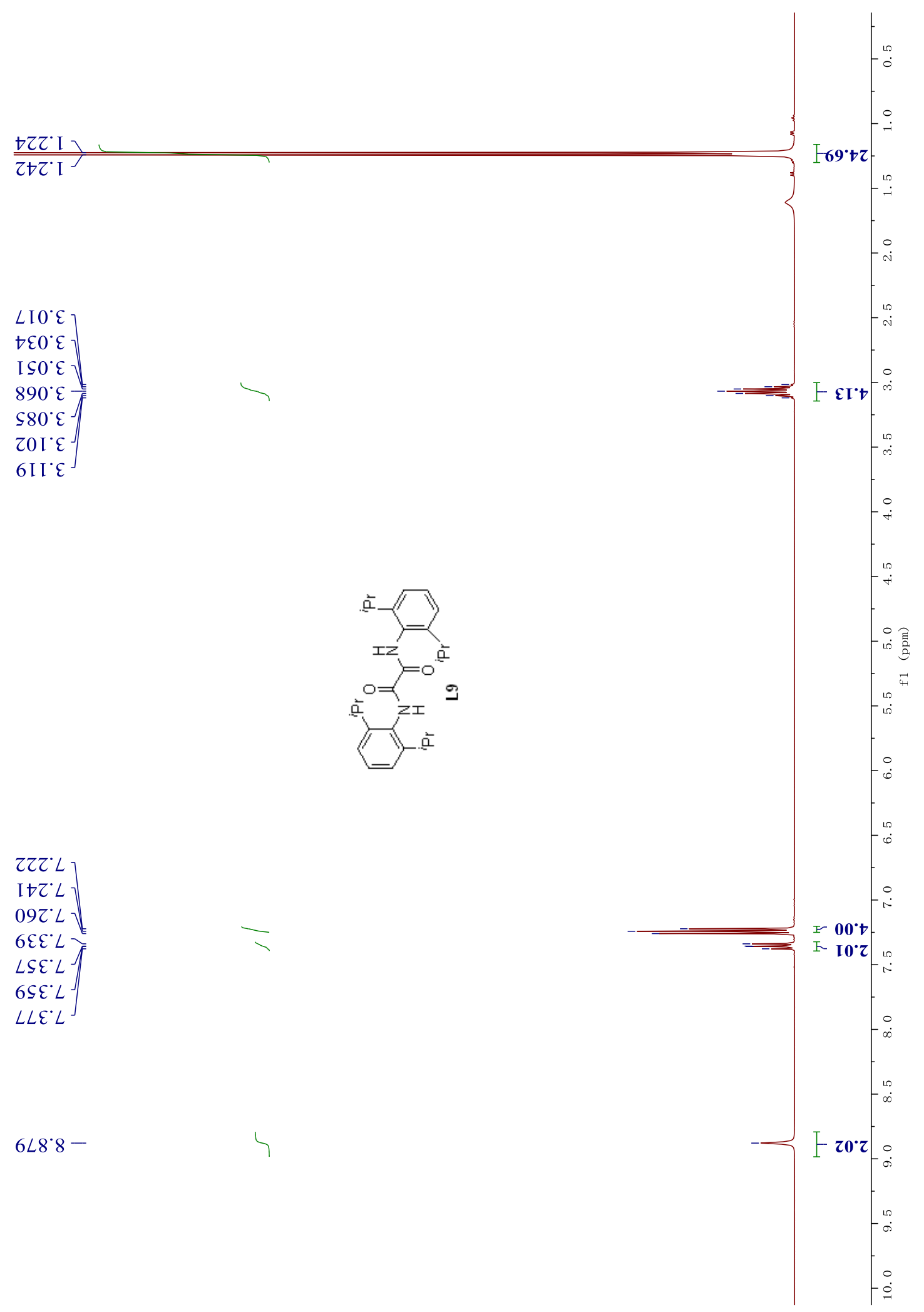


I L L $\mathcal{E} \varepsilon$

ยIZ・6Z-

$\varepsilon \nabla 8^{\circ} 9 L$

$09 I^{\circ} L L \frac{1}{]}$

$8 \angle D^{\circ} L L$

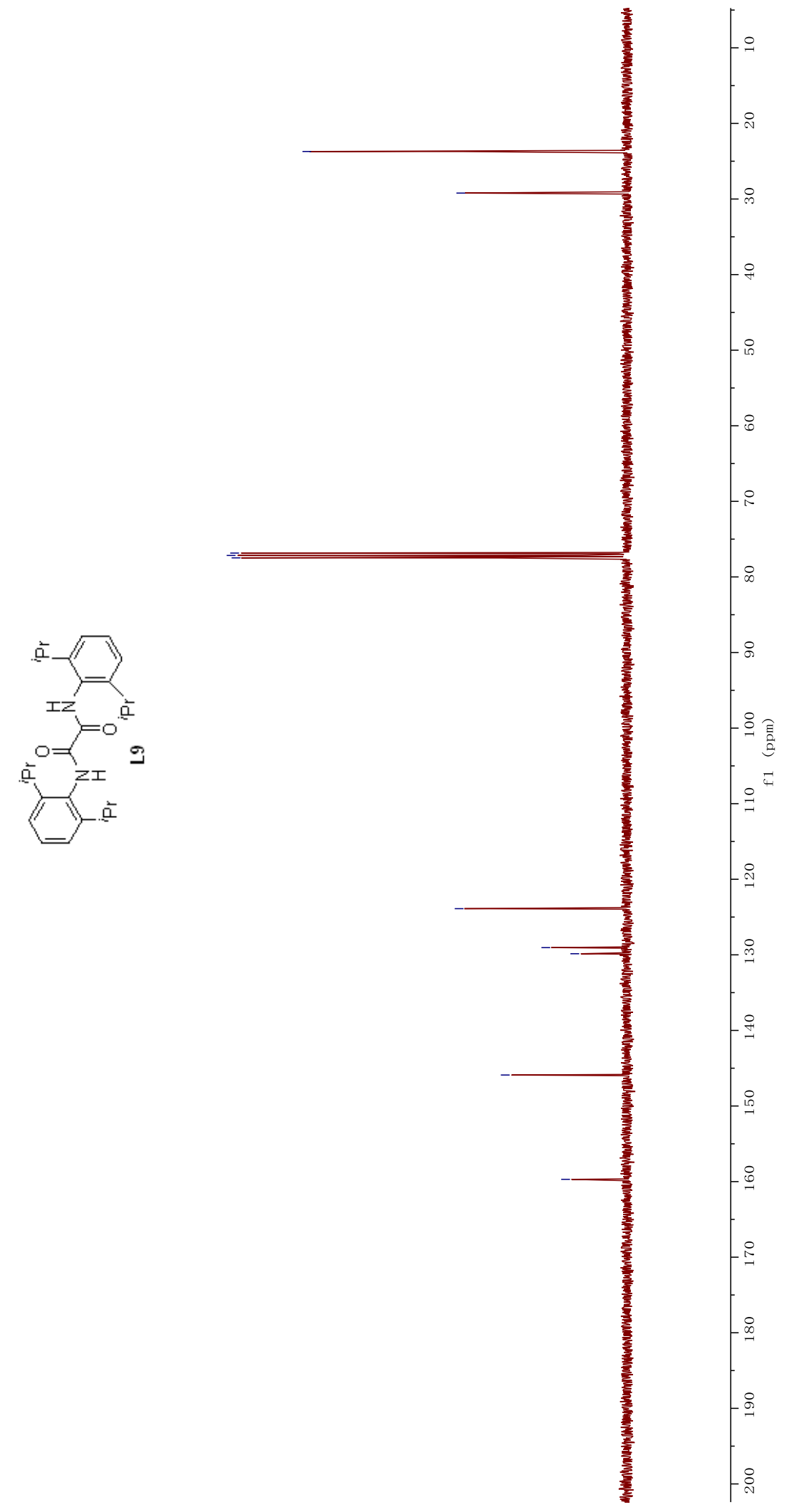

$068^{\circ} \varepsilon Z I \sim$

Z†0.6ZI

$\varepsilon \varsigma 8^{\circ} 6 \mathrm{ZI}^{-}$

L68 st I -

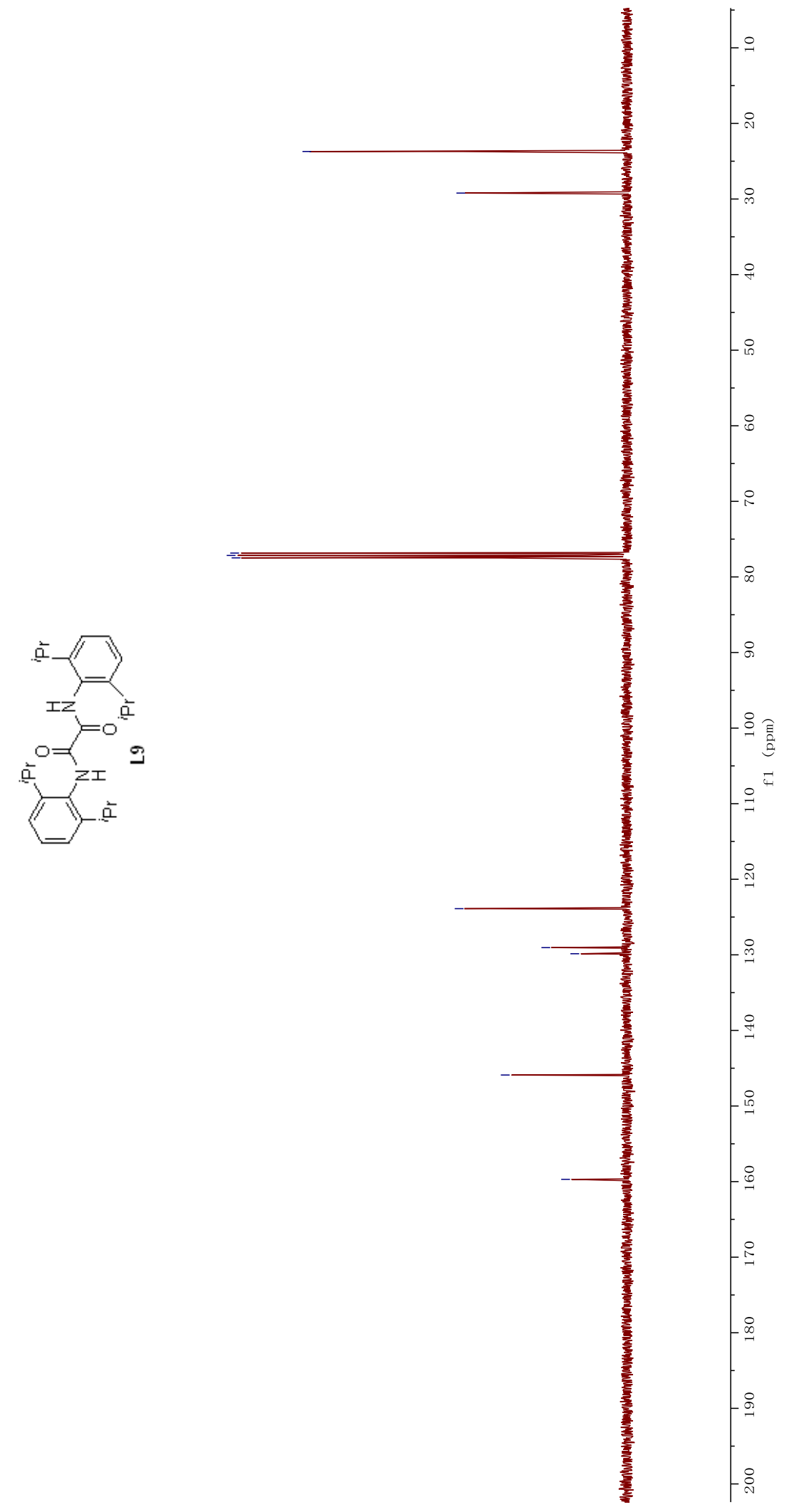

$00 L^{\circ} 6 S_{\text {I }}-$ 


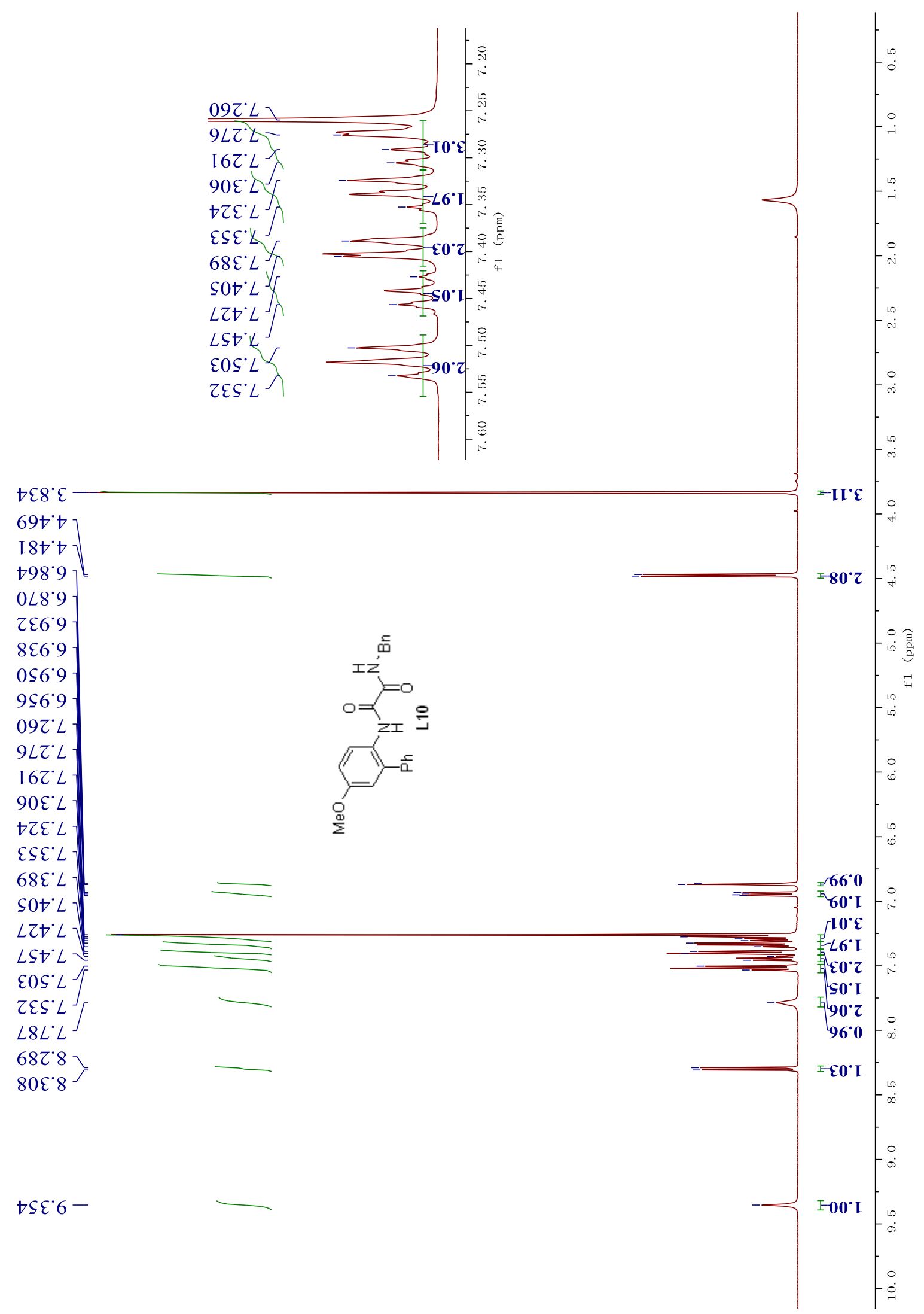




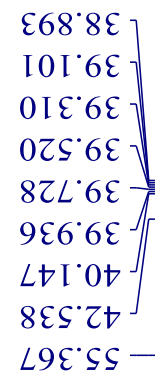

LZS $\varepsilon I I$

ऽEZ ${ }^{\circ} I I$

$\varsigma 9 Z^{\circ} \varsigma Z \mathrm{I}$

I09.9Z I

$626^{\circ} 9 \mathrm{ZI}$

$6 \mathrm{I} \varepsilon \cdot L Z \mathrm{I}$

$8 \varepsilon L^{\circ} L Z \mathrm{I}$

99Z'8Z I

IS9.8Z I

ZEL'8ZI

I $8 L^{\circ} 9 \varepsilon \mathrm{I}$

ZI0.8EI 广

†IS.8EI

$780^{\circ}$ LS I

$9 \mathrm{II} \cdot 8 \mathrm{SI}$

SL8.6SI

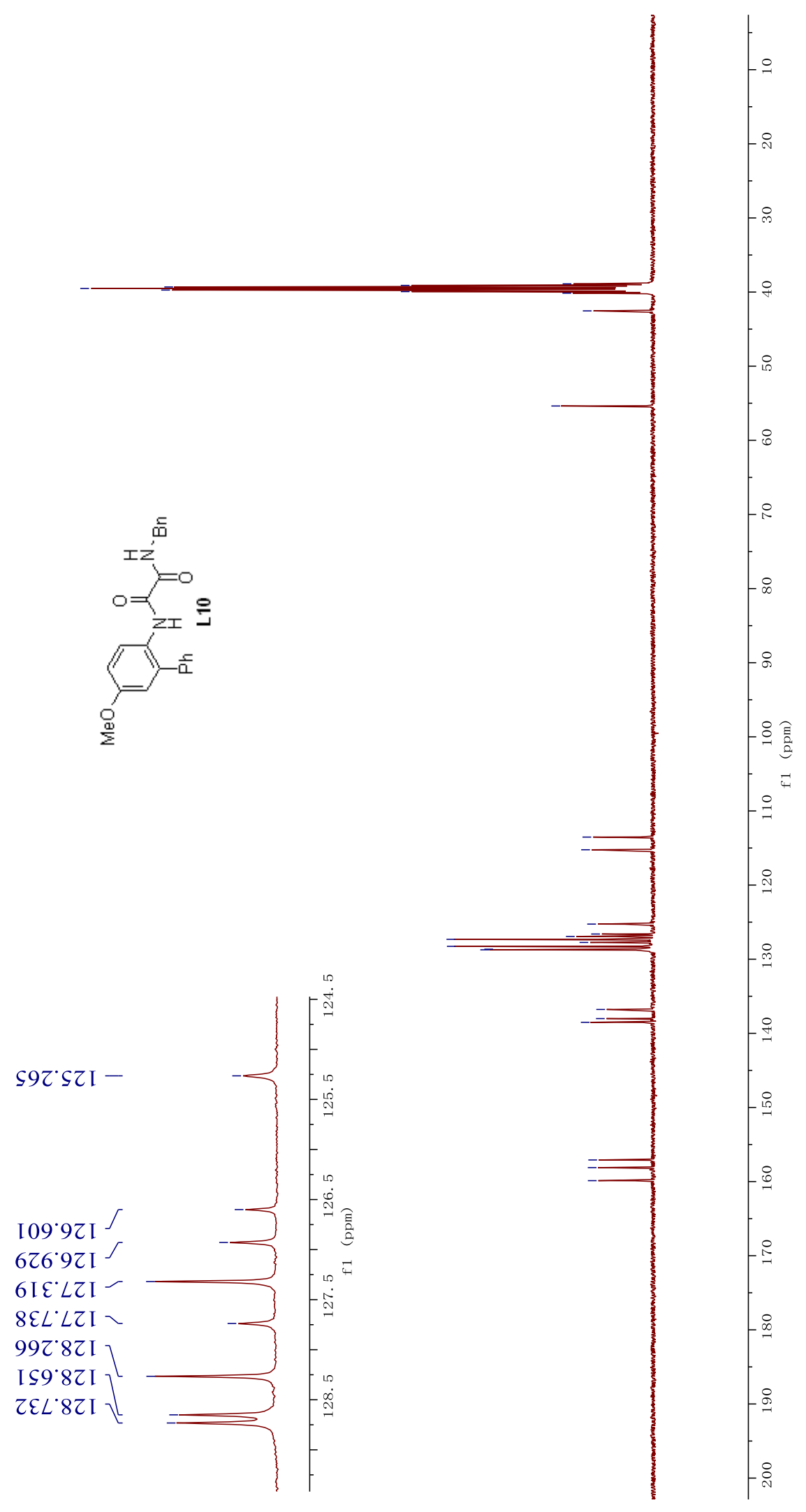


I†でてー
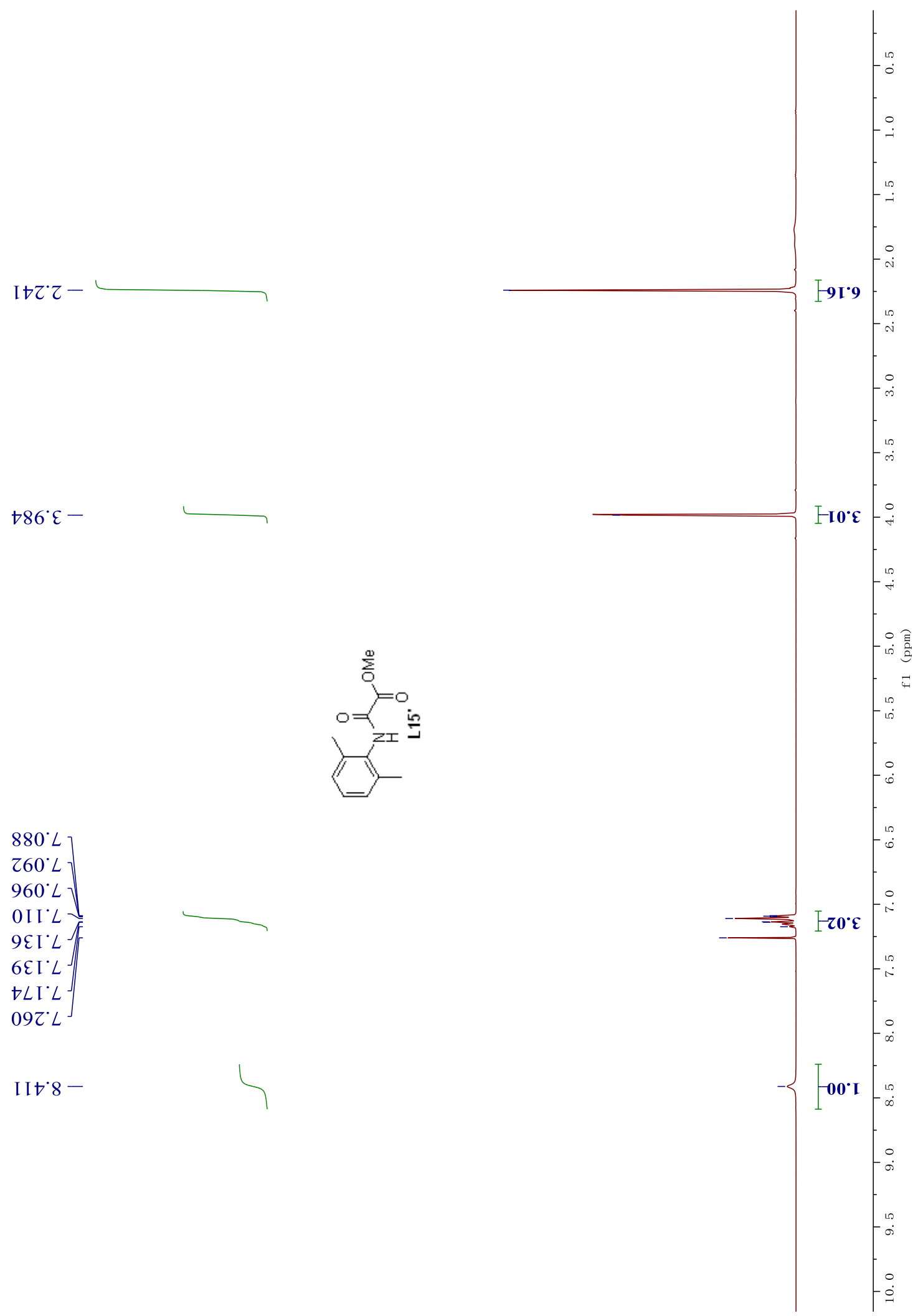
9 IE:8I-

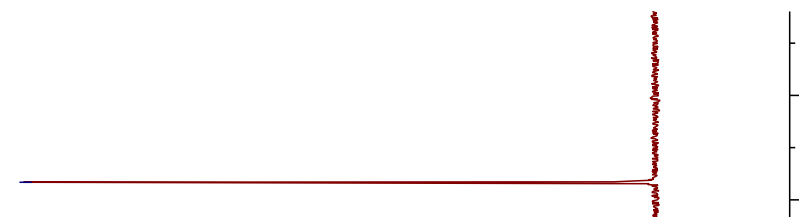

L9L'ES -

$2 \downarrow 8^{\circ} 9 L$

$09 I^{\circ} L L \frac{1}{5}$

$6 \angle \nabla^{\circ} L L$

$\neg Z 6^{\circ} \mathrm{LZI}$

$88 \mathrm{Z}^{\circ} 8 \mathrm{ZI}^{\prime}$

6S0 $2 \varepsilon \mathrm{I}$

$096^{\circ} \nabla \varepsilon$ I

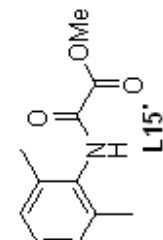

용

운

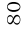

음

†0ฑ๋ ธI -

6ยZ' [9I -

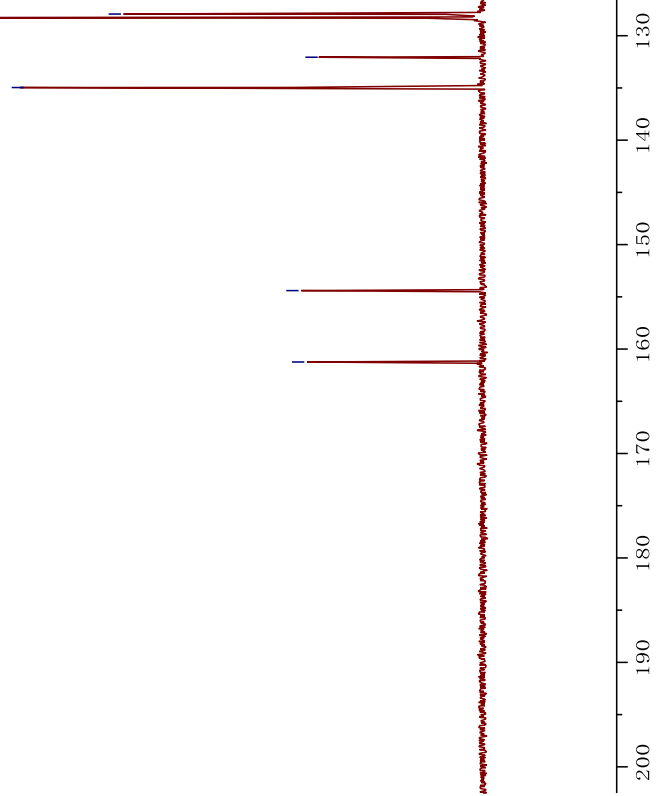



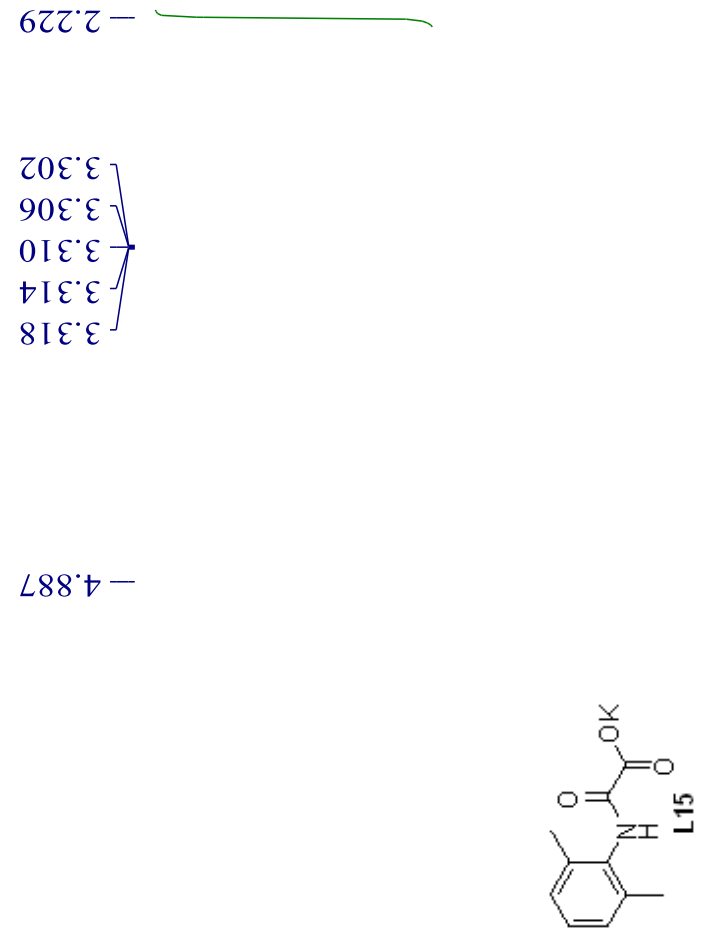

$080^{\circ} \mathrm{L}$

$280^{\circ} \mathrm{L}$

$680^{\circ} \mathrm{L}$

[ $60^{\circ} \mathrm{L}$ ]

$660^{\circ} \mathrm{L}$

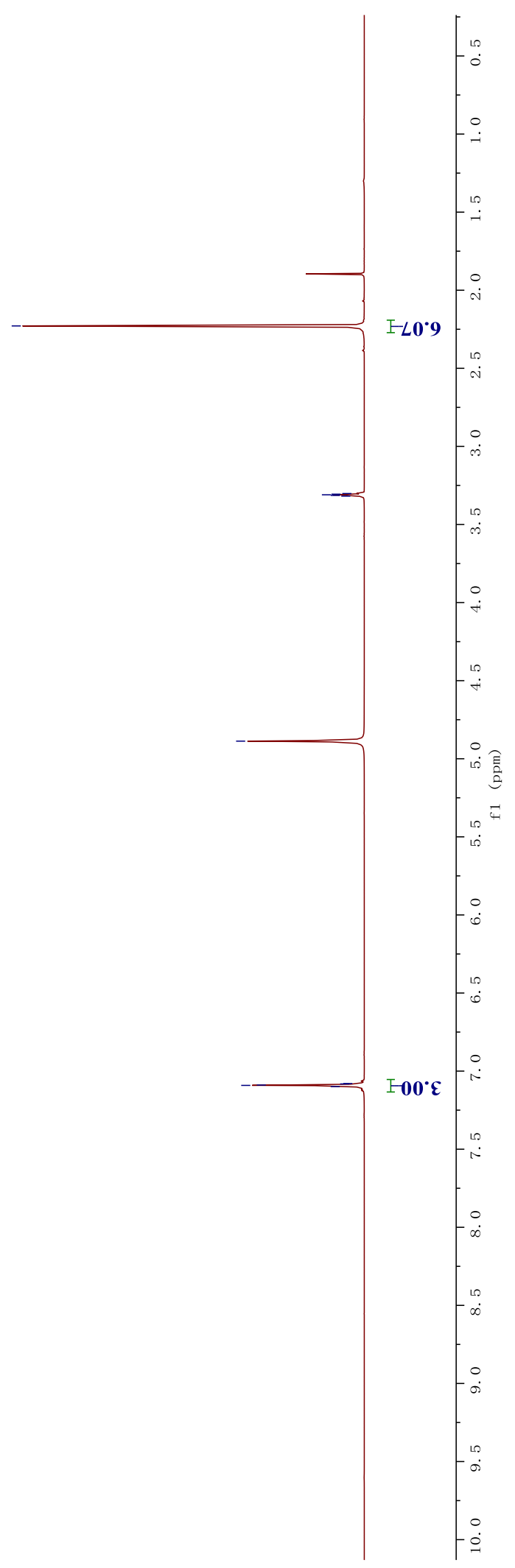


2ZS:8I-

[9ع:8t

$\checkmark \angle S^{\circ} 8 \mathrm{t}$

$\angle 8 L \cdot 8 t$

$000^{\circ} 6 t$

$\varepsilon I \tau \cdot 6 t$

97t $6 t$

$6 \varepsilon 9^{\circ} 6 t$
Z0E.8ZI

$\angle 00{ }^{\circ} 6 Z \mathrm{I}$

0IE $\subseteq \mathcal{~ I ~}$

$8 \nabla \varsigma^{\circ} 9 \varepsilon \mathrm{I}$

090 $99 \mathrm{I}$

ISE⿱ 99 I $^{\top}$

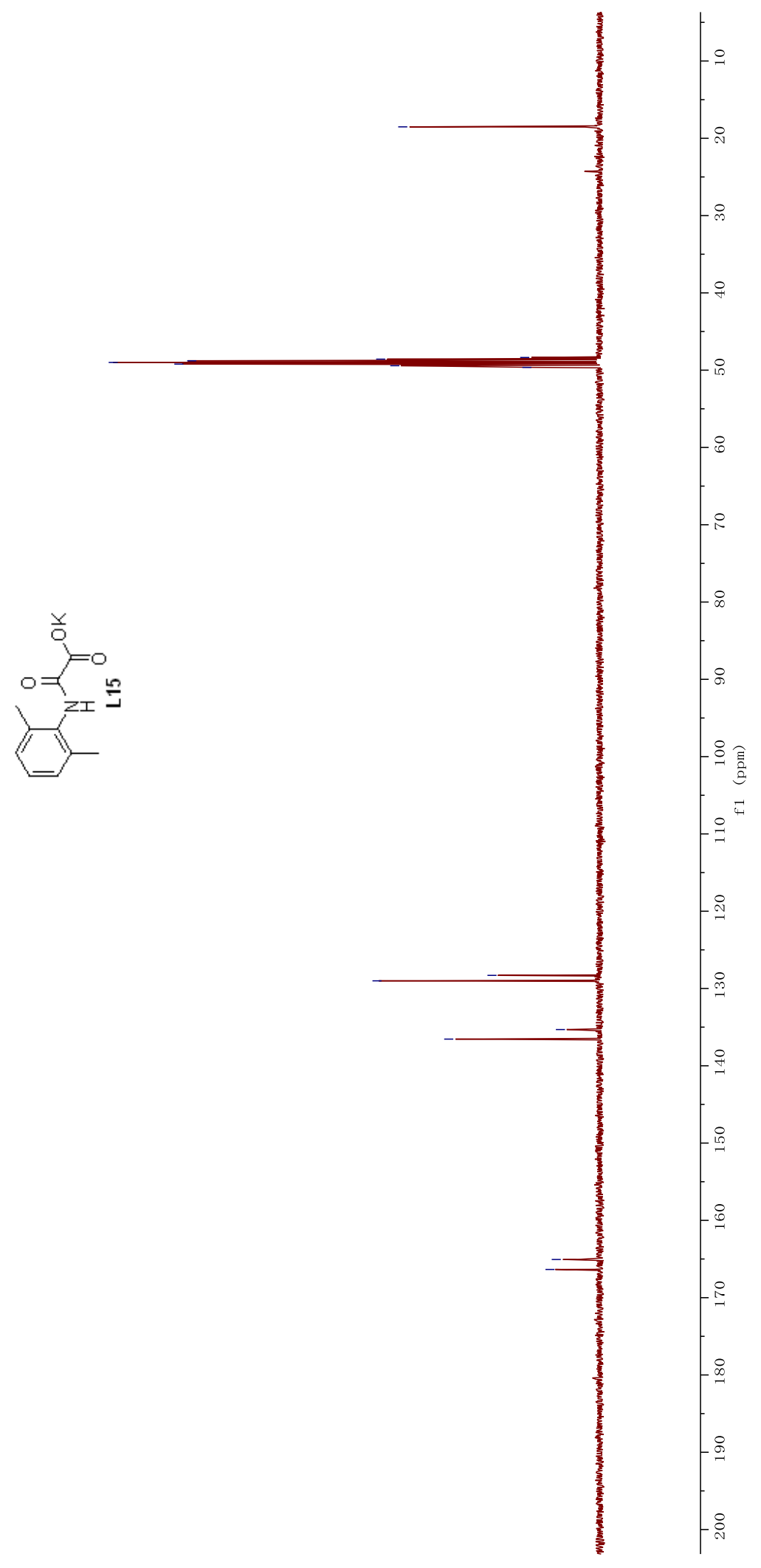



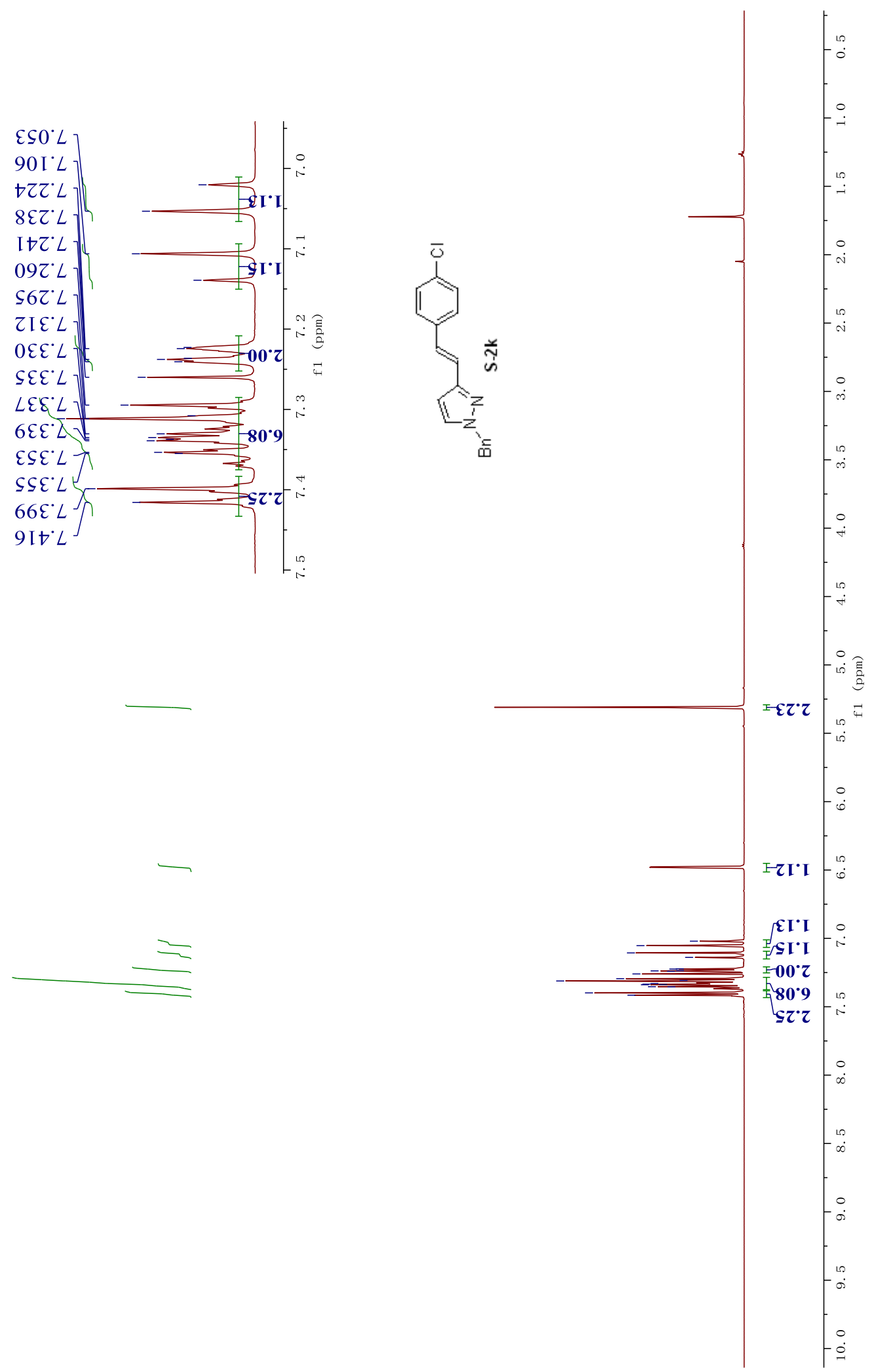


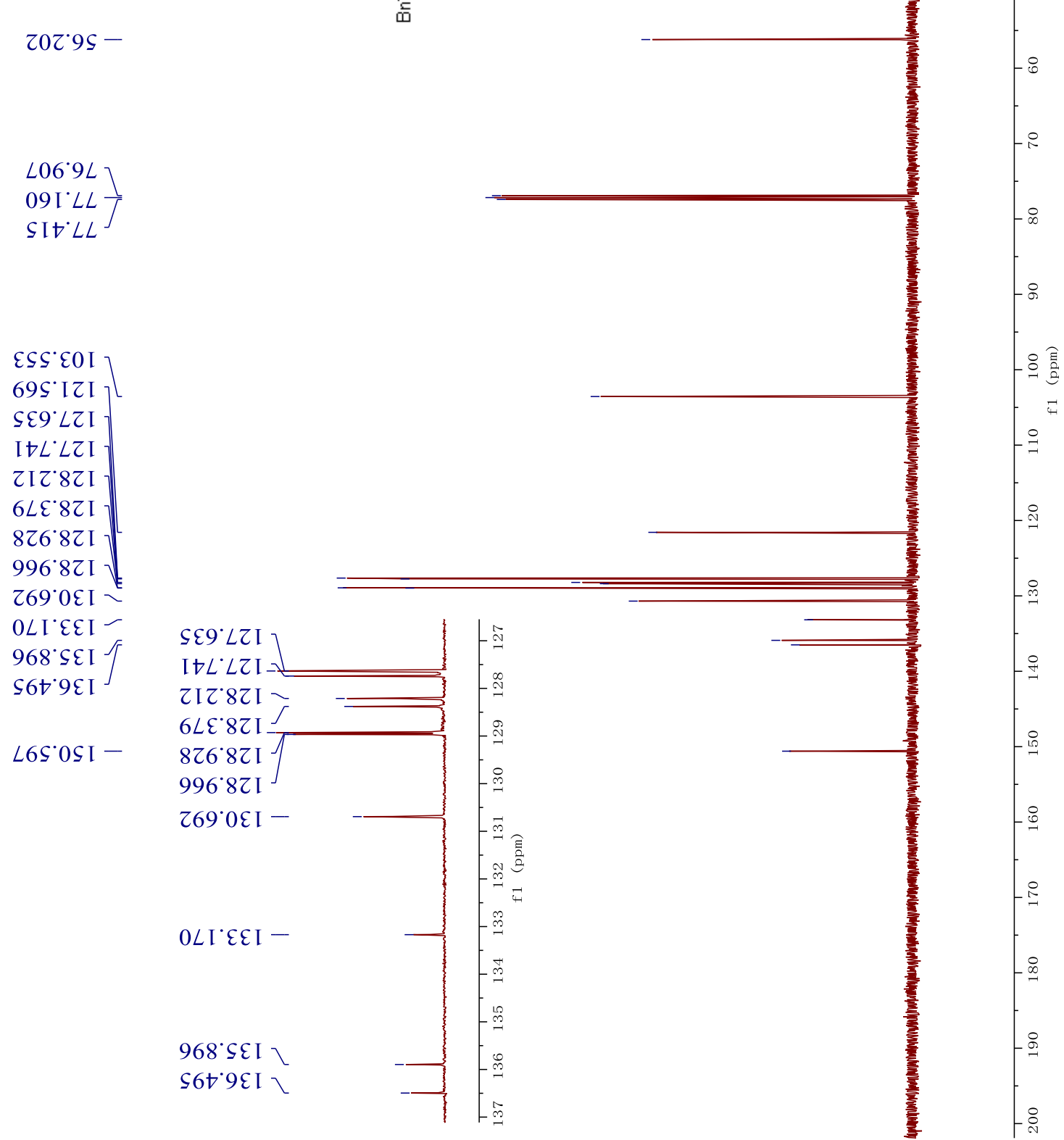




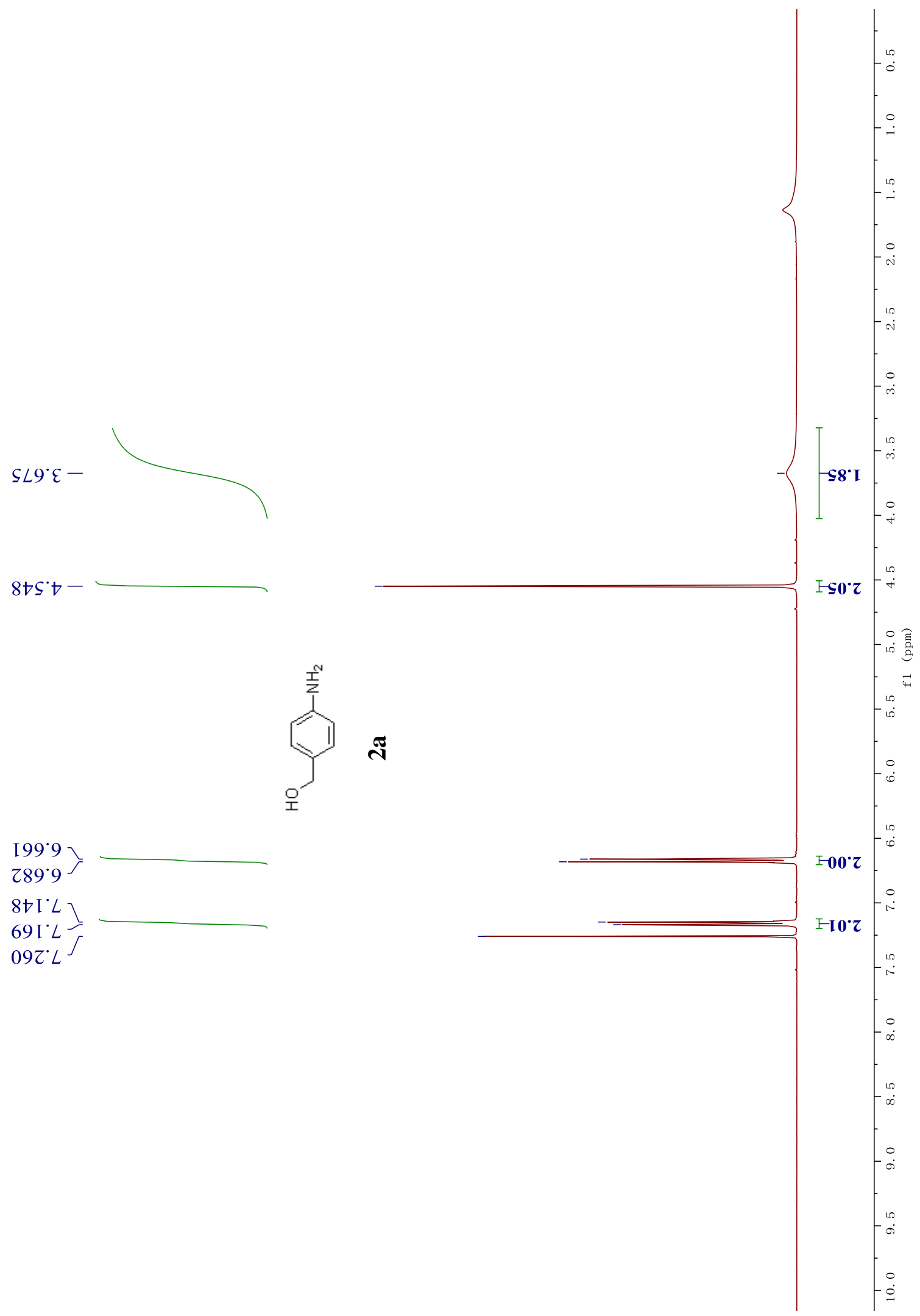


zoE $\$ 9-$

$906^{\circ} 9 L$

$09 I^{\circ} L L \frac{1}{J}$

SIt $L L$

Z†て`SII -

$\angle \supset 8^{\circ} 8 Z \mathrm{I} \sim$

I L I' IE I -

$\stackrel{0}{\stackrel{1}{a}}$

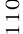

$280^{\circ} 9$ t I - 


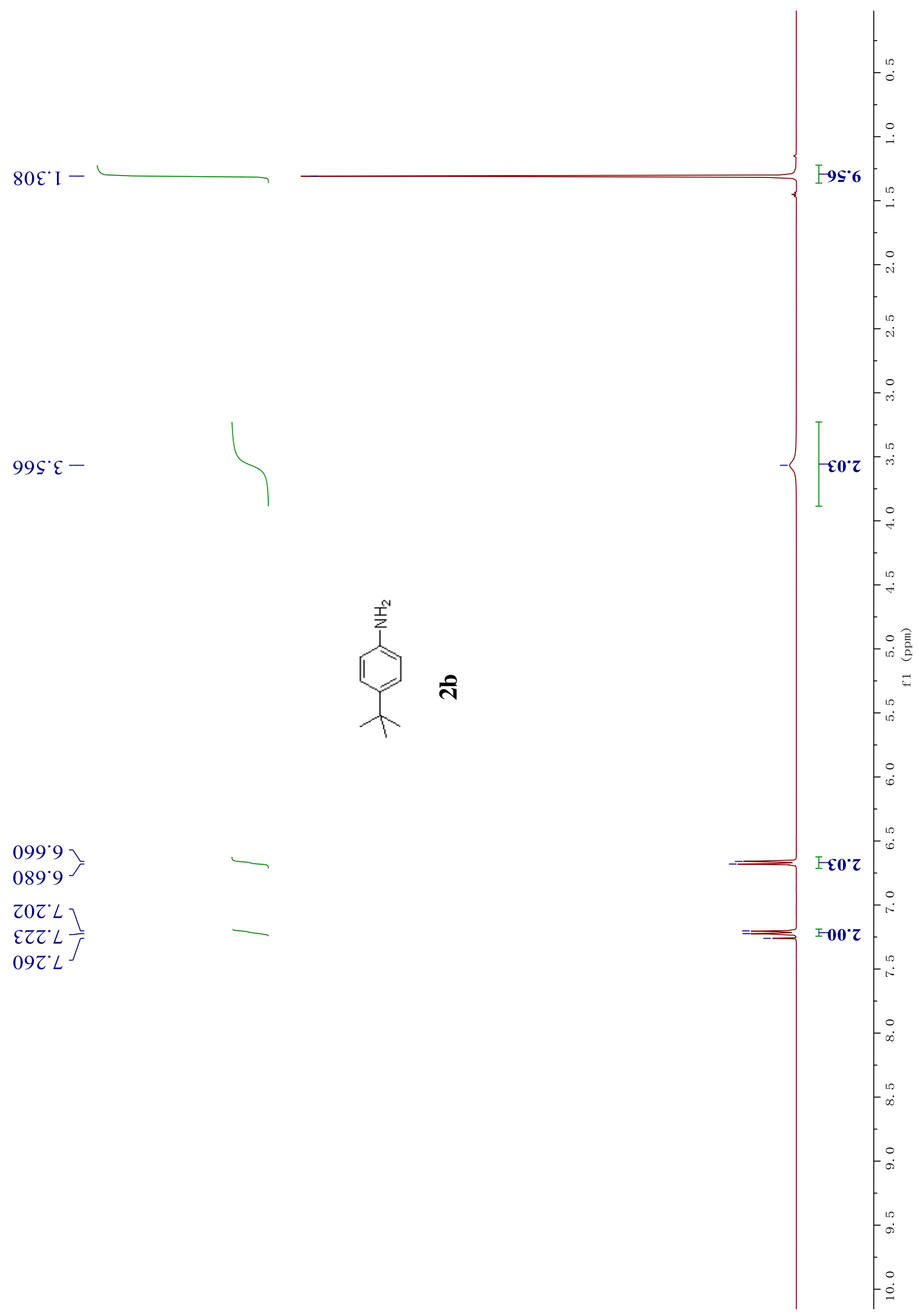


Eเวดว $878^{\circ} 9 L$

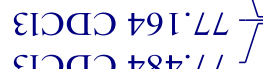

SZS IE $~$ $628^{\circ} \varepsilon \varepsilon-$

$0 \angle 8^{\circ} \nabla$ I I -

† $6^{\circ}$ ऽ I -

LZI'It I

으

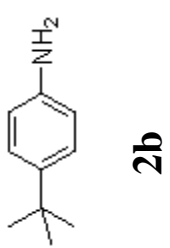

$-ㅇ$

$628^{\circ}$ \&力 I -

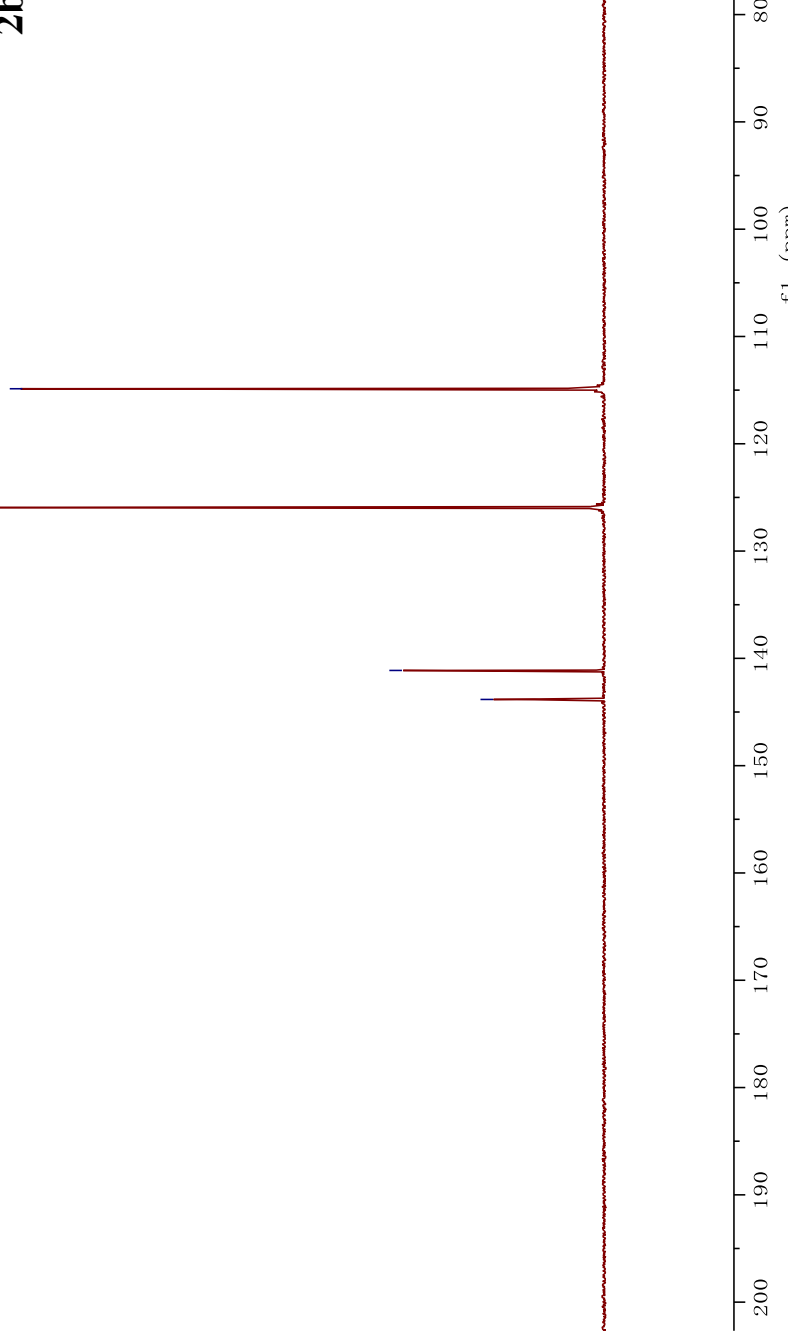




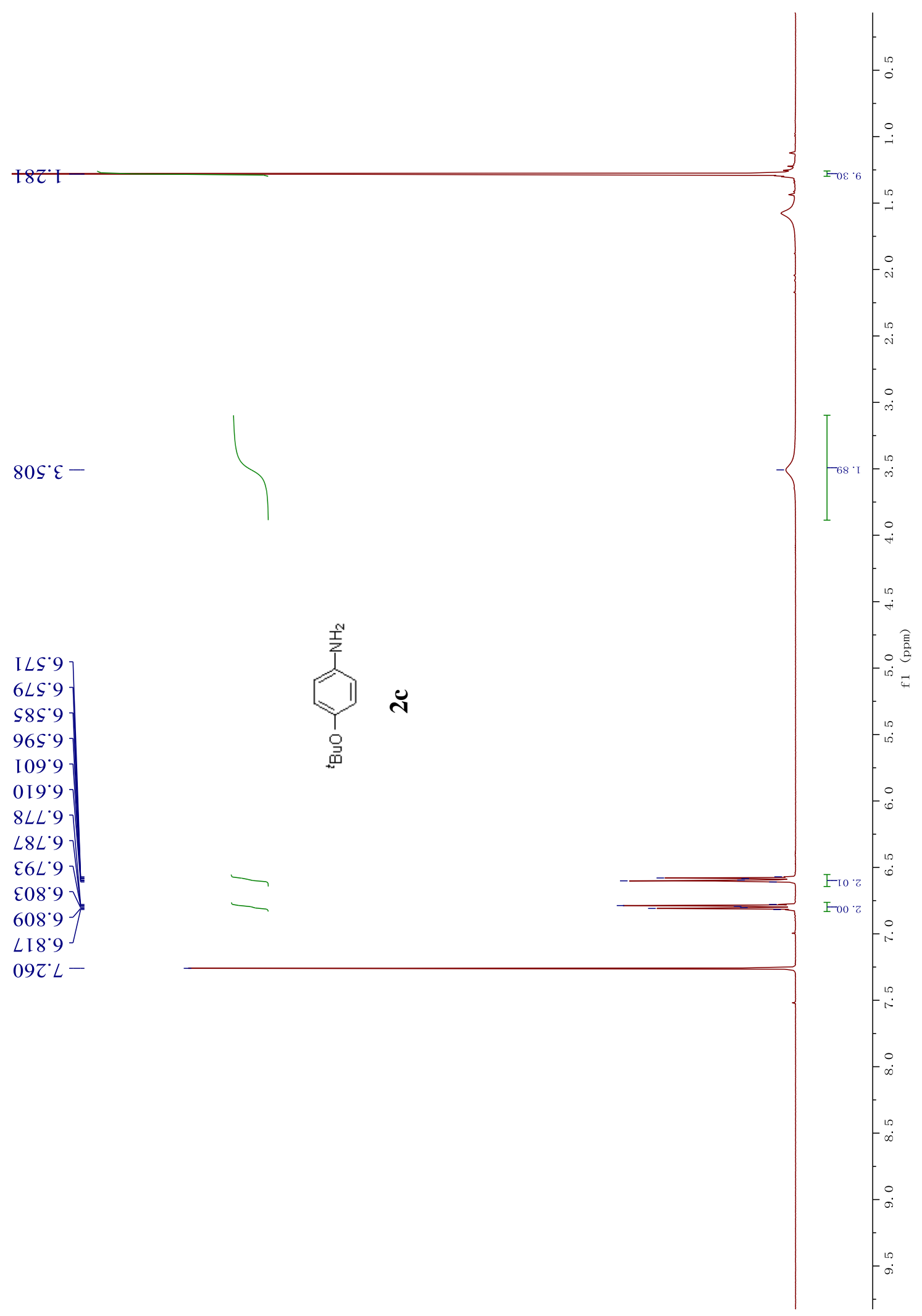




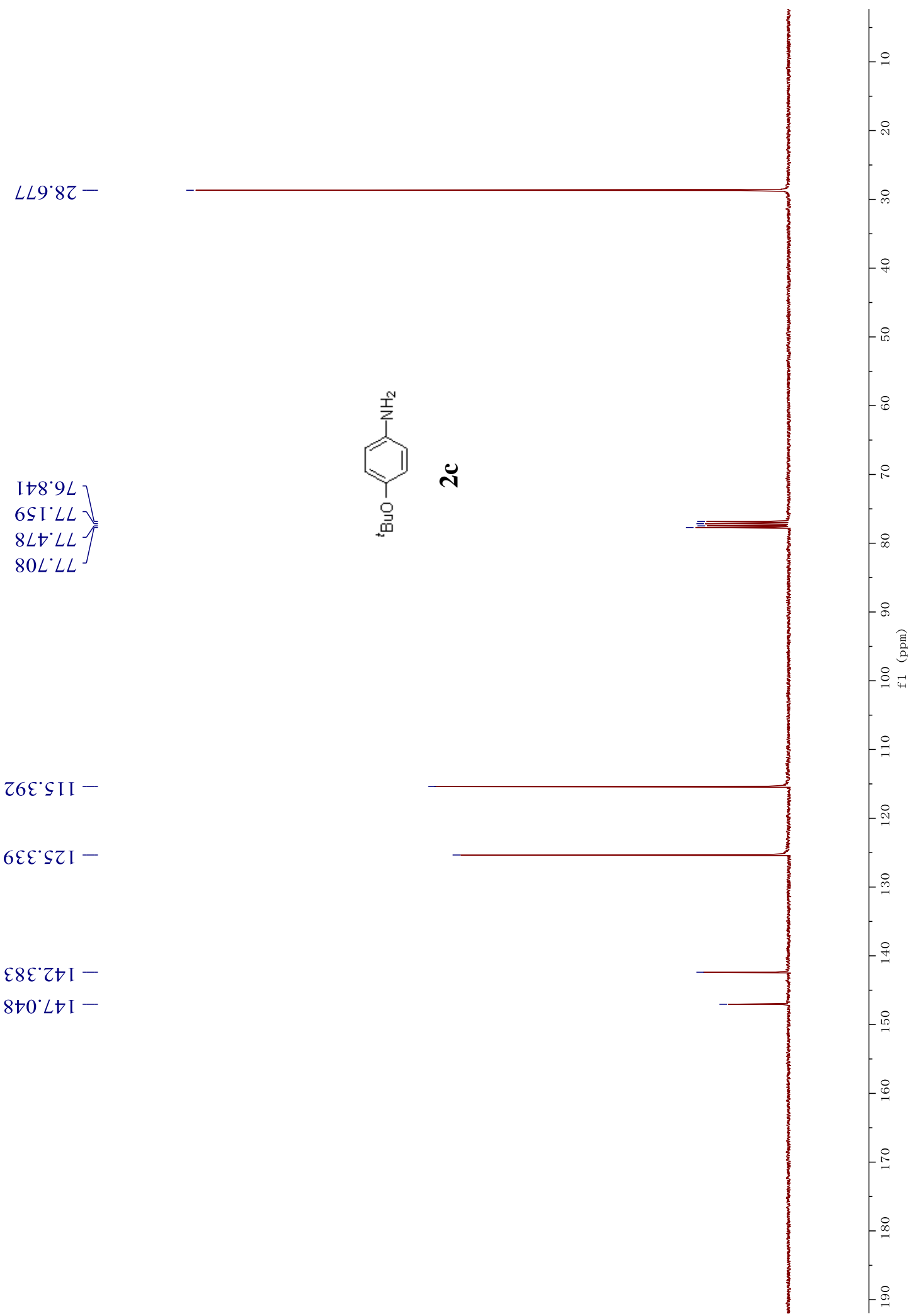




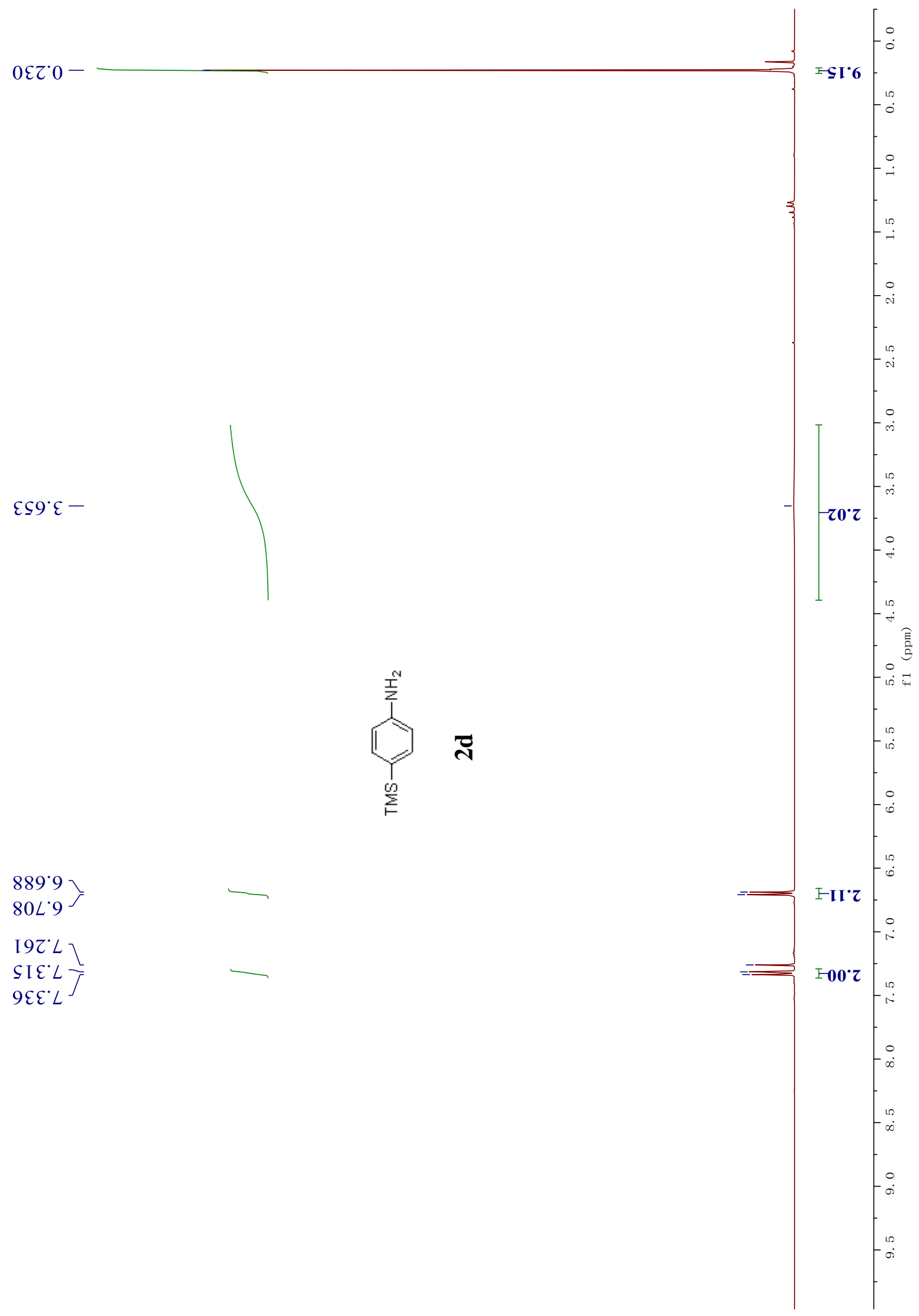


$\nabla L L^{\circ} 0^{-}-$

$\varepsilon \oplus 8^{\circ} 9 L$
$\left.09 I^{\circ} L L\right]$

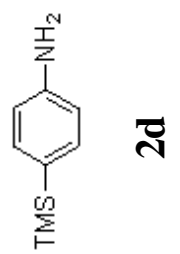

Z69` I I -

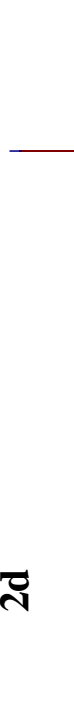

$8 \angle \nabla^{\circ} L L$

てカナ・8ZI -

$\neg \varepsilon 9^{\circ} \supset \varepsilon \mathrm{I}-$

$\varepsilon \varepsilon I^{*} L \dagger I^{-}$

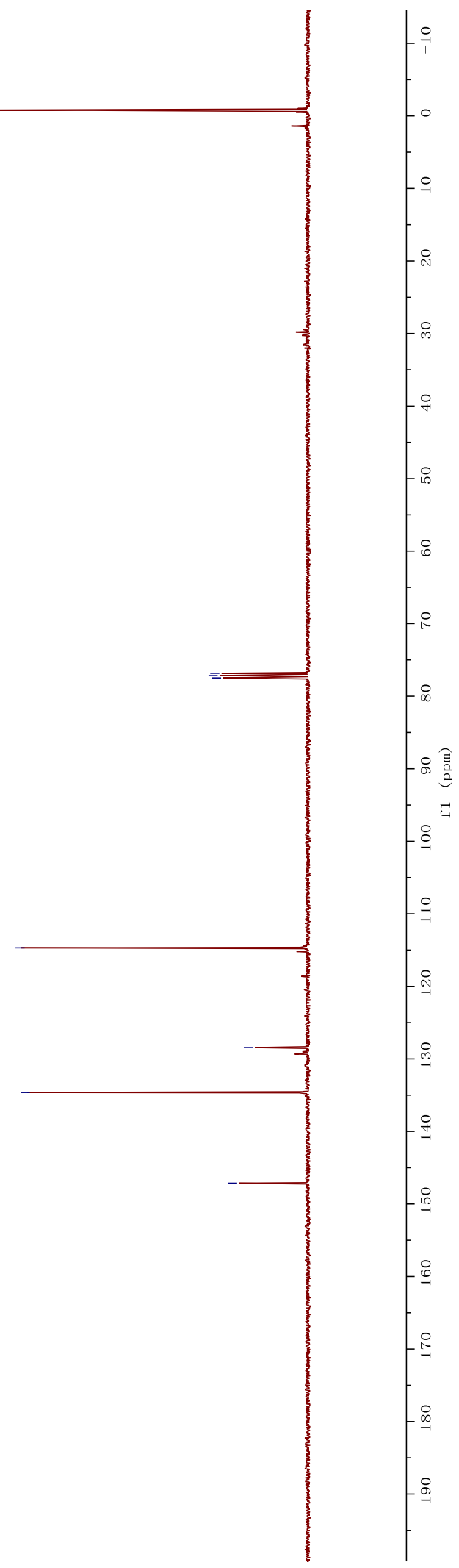




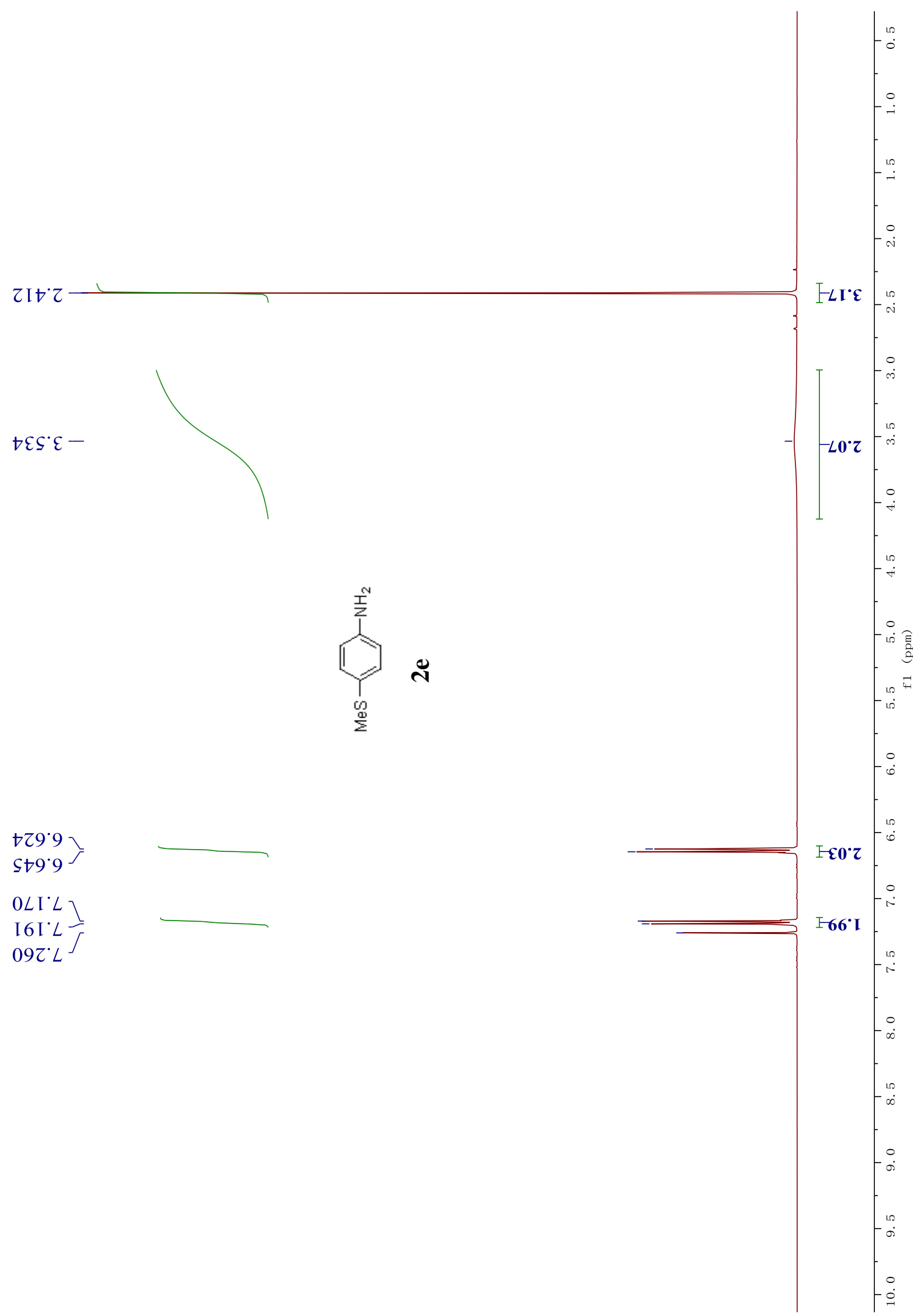


$99 L 8$ I -

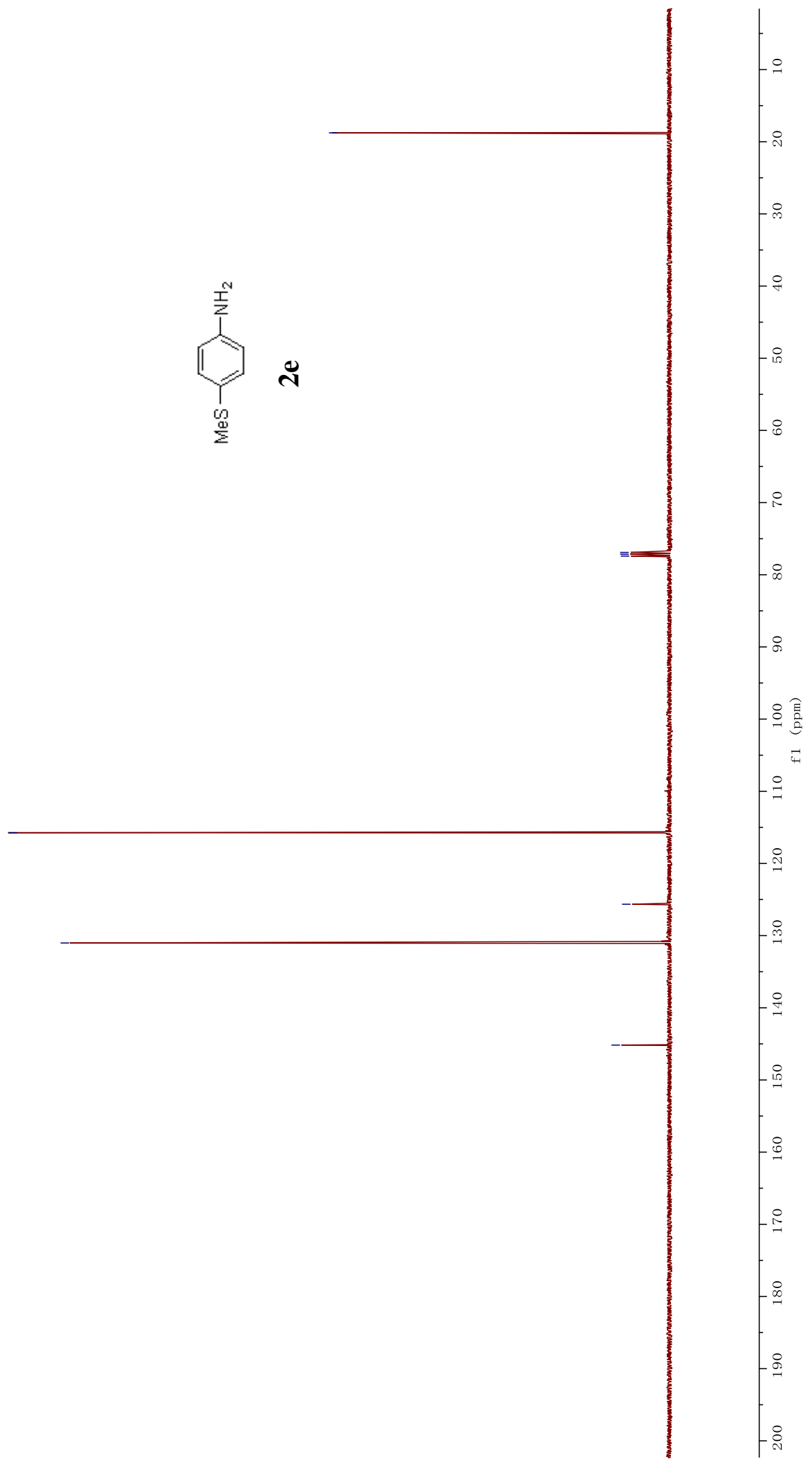

$\left.\begin{array}{l}\subseteq 06^{\circ} 9 L \\ \left.L S I^{\circ} L L\right] \\ \varepsilon I t^{\circ} L L\end{array}\right]$

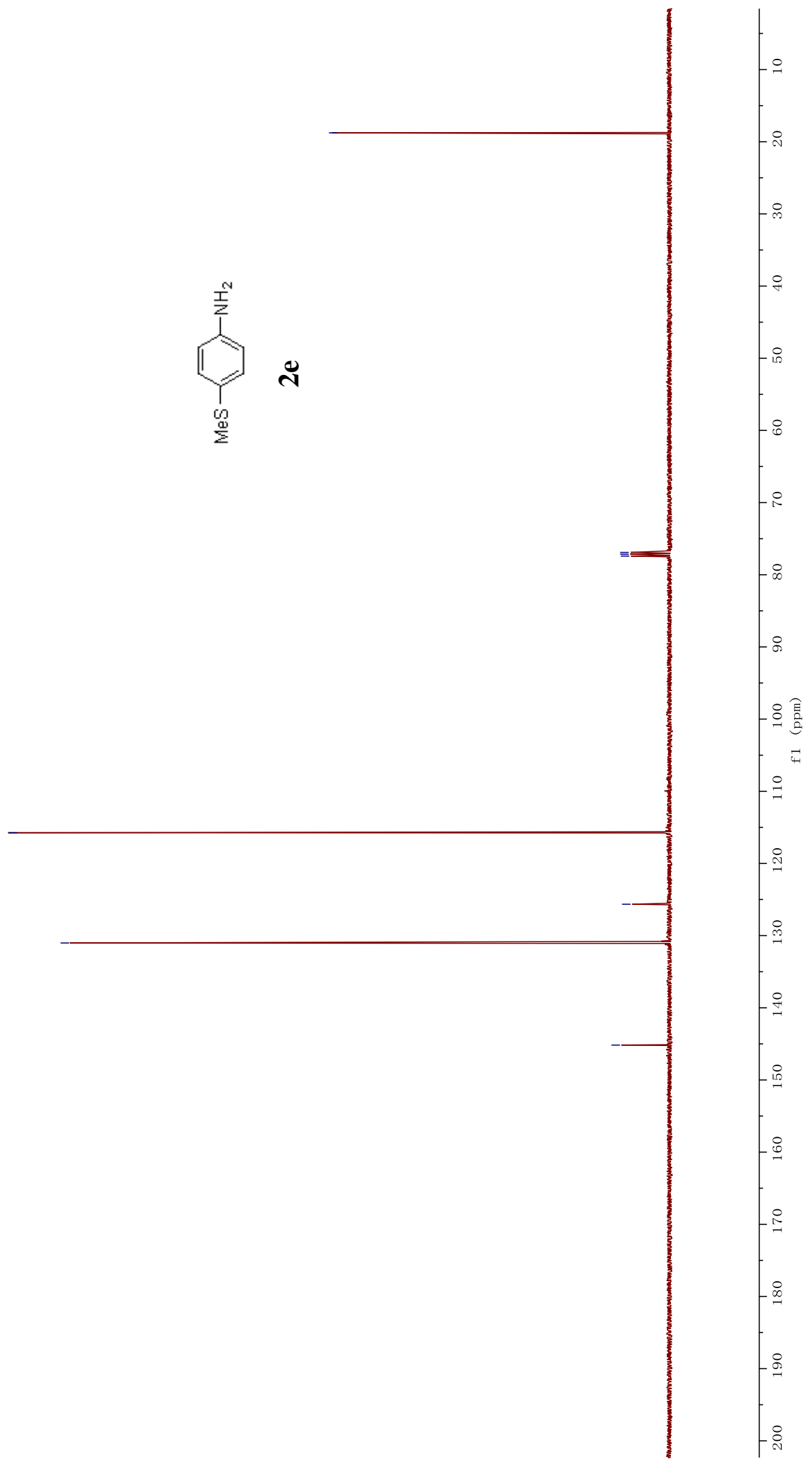

99281

$8 \mathcal{E L}$ S II -

Zऽ9.`ZI -

6I0.IEI -

L9I S D I - 

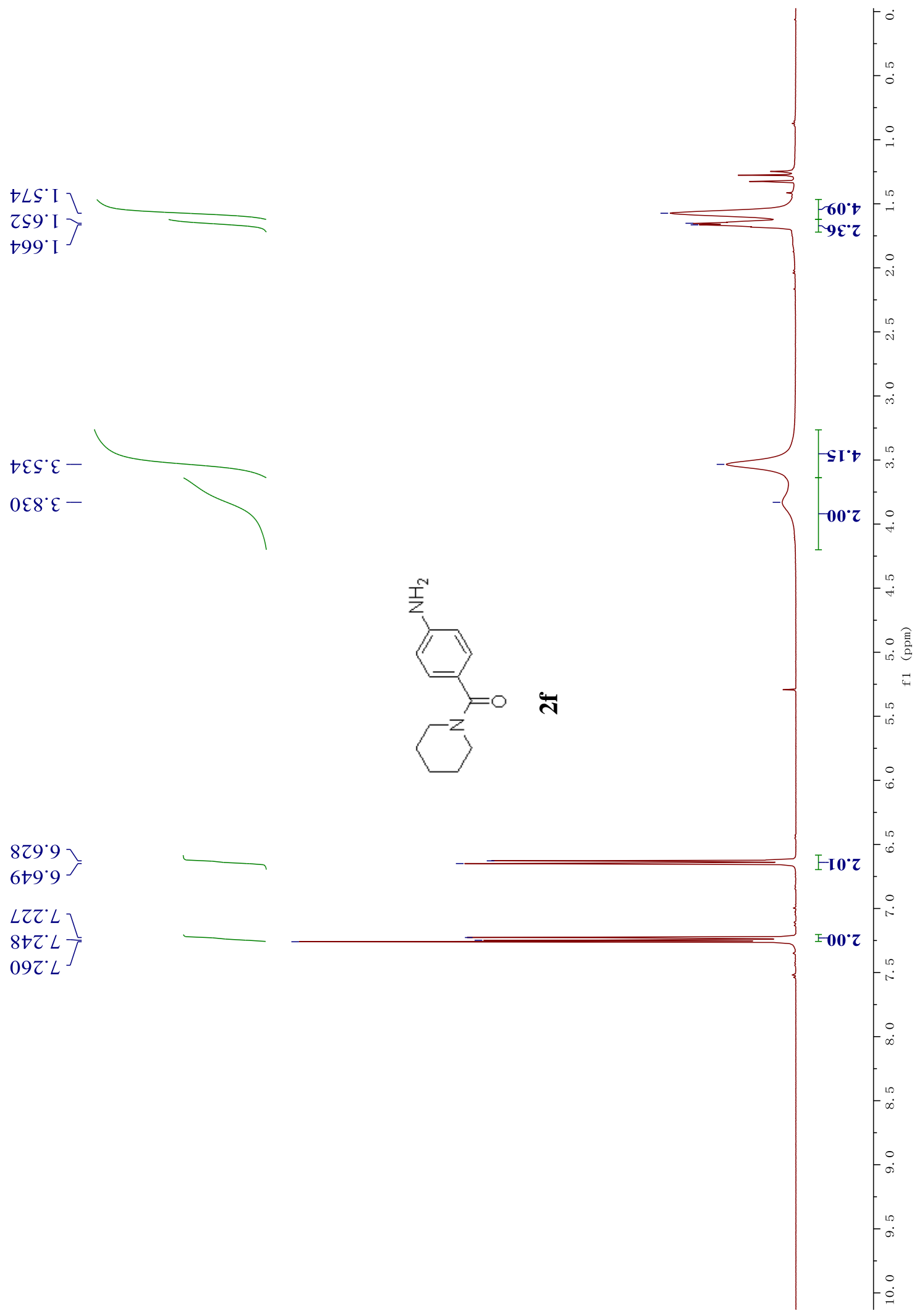

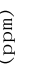

$\downarrow \varepsilon \varsigma^{\cdot} \varepsilon-$

$0 \mathcal{E} 8^{\cdot} \mathcal{E}-$

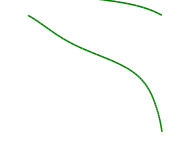

$879^{\circ} 9$
$6+9^{\circ} 9^{J}$
$\angle 2 Z^{\circ} L$
$8 \nabla Z^{\circ} L$
$09 Z^{\circ} L$
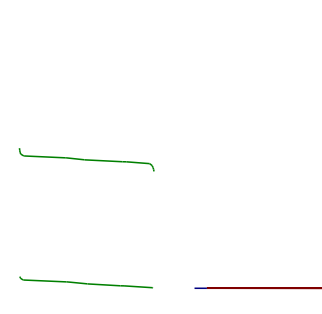
I6で๑て

$\angle S L^{\circ} S Z^{\prime}$

$0 † 8^{\circ} 9 L$

$09 I^{\circ} L L T$

$009^{\circ} \mathcal{E}$ I I -

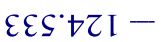
09ऽ $8 Z \mathrm{I}$

ऽZE'8tI -

$209^{\circ} 0 L \mathrm{I}-$

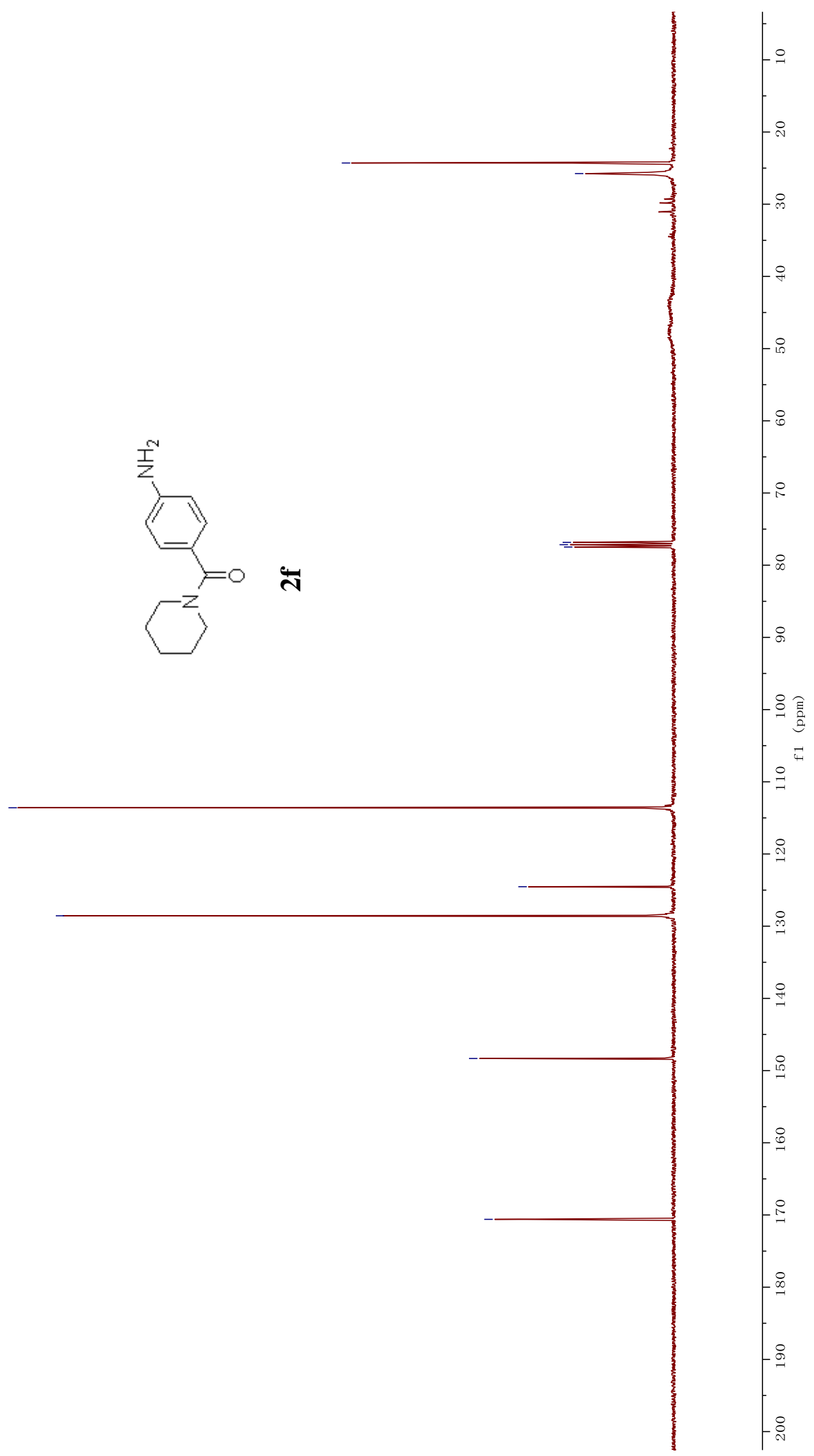




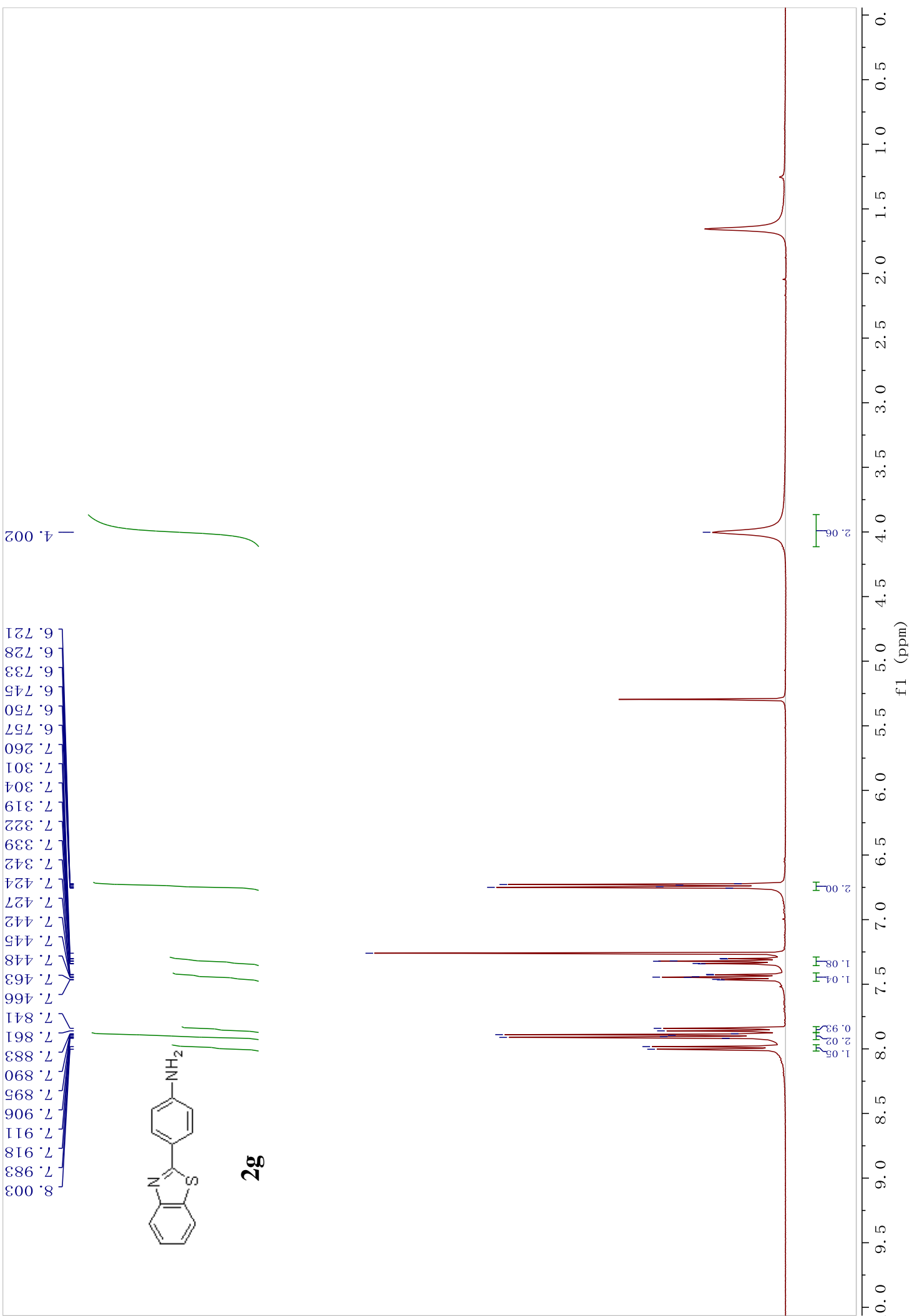




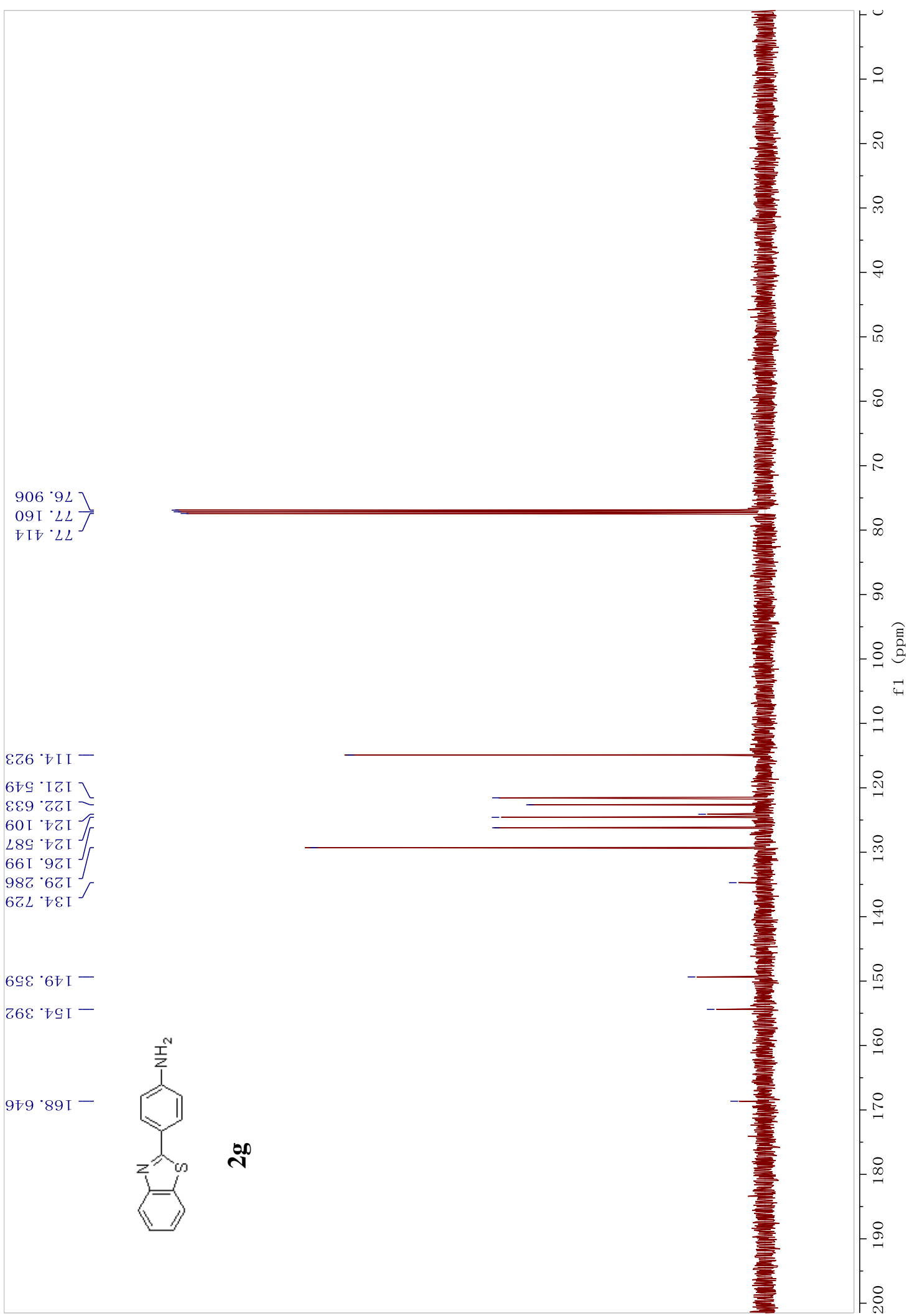




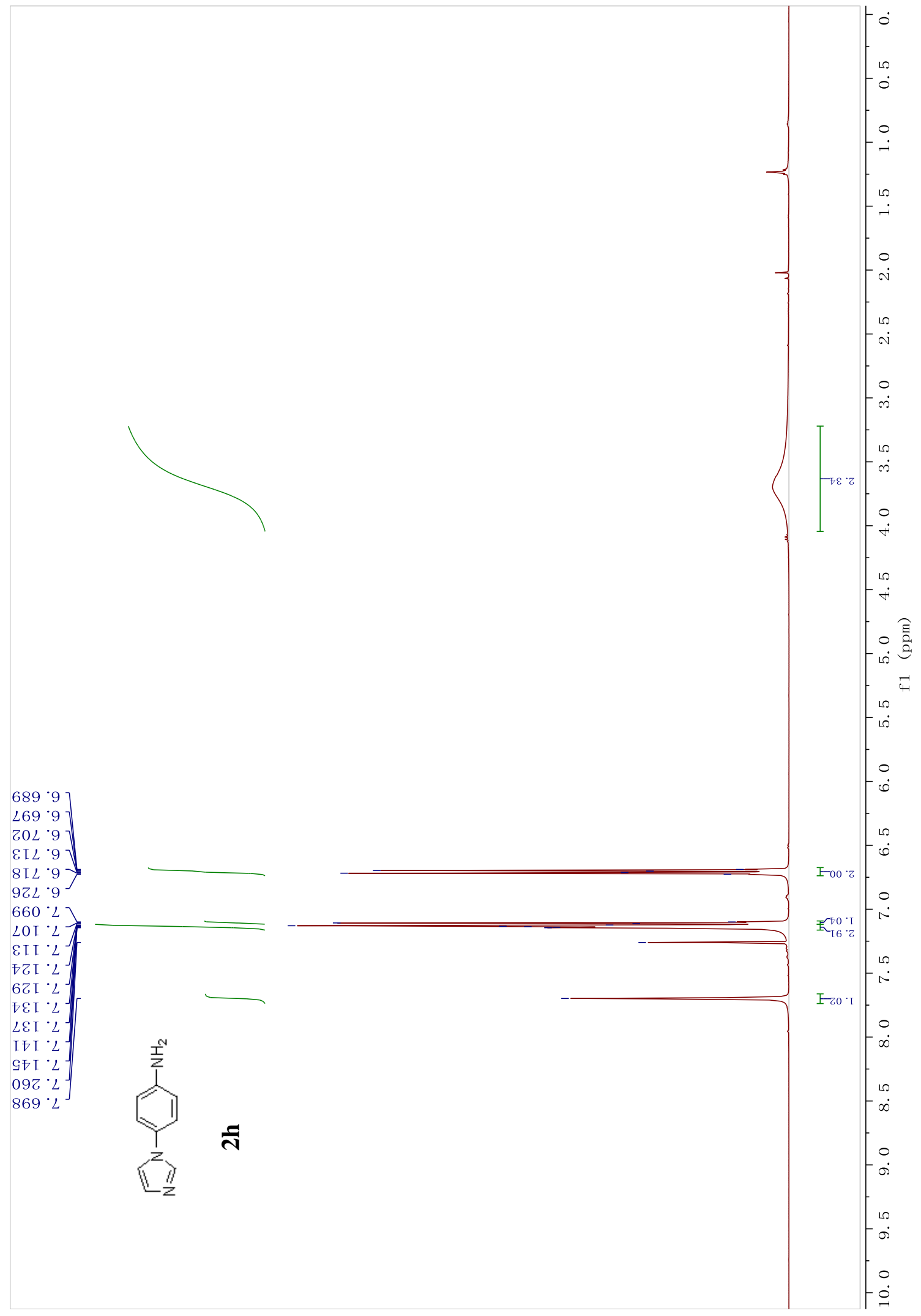




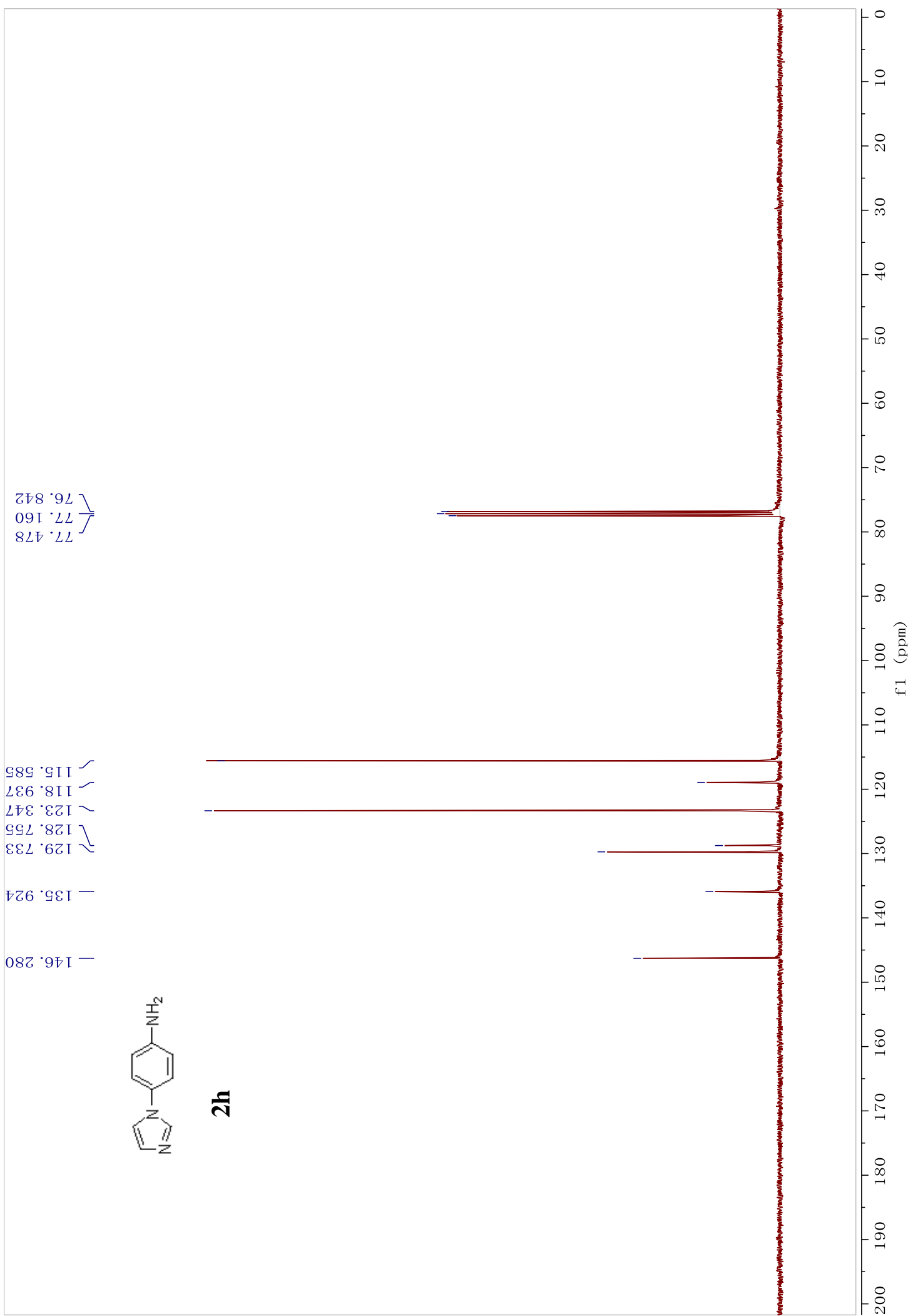




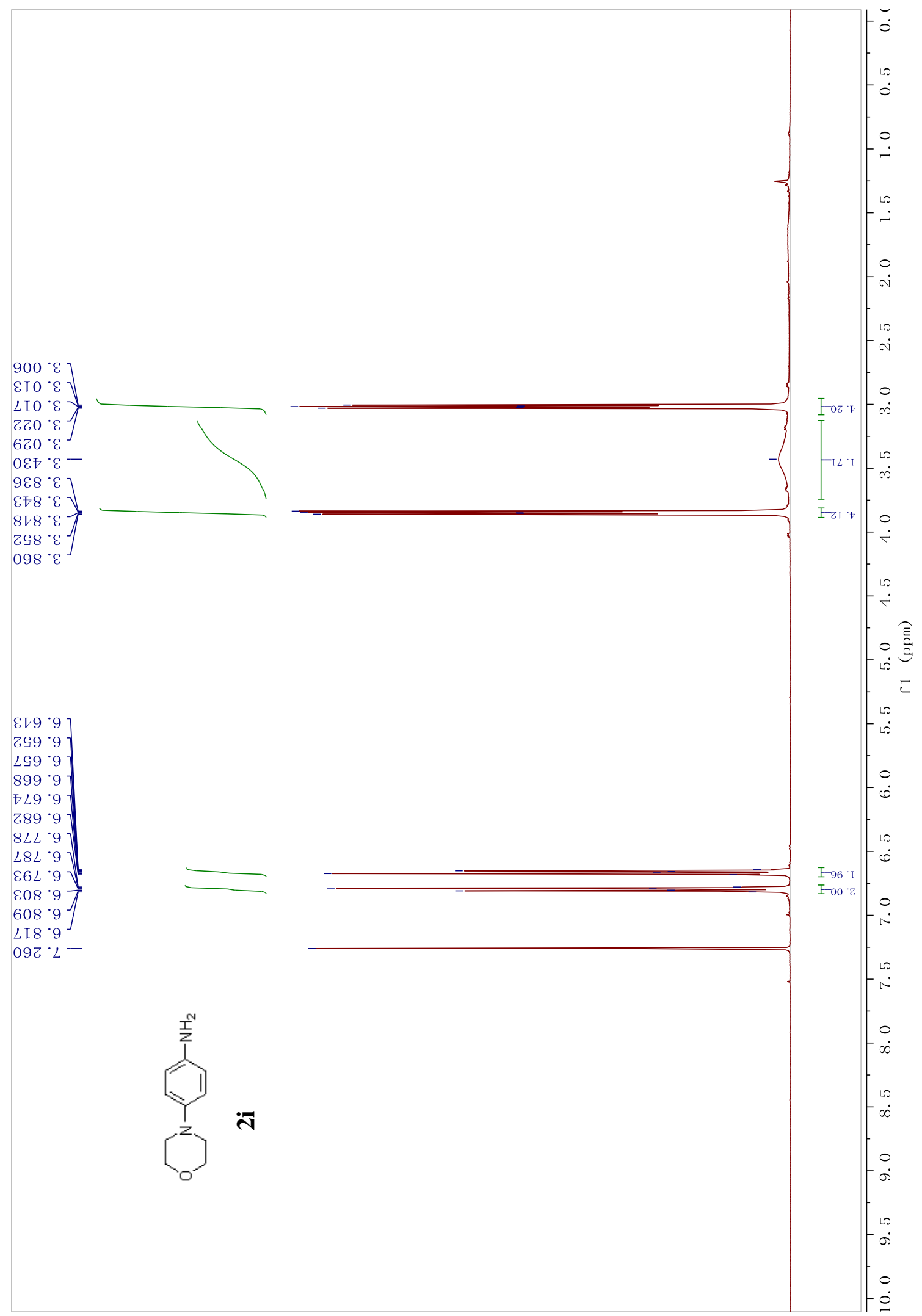




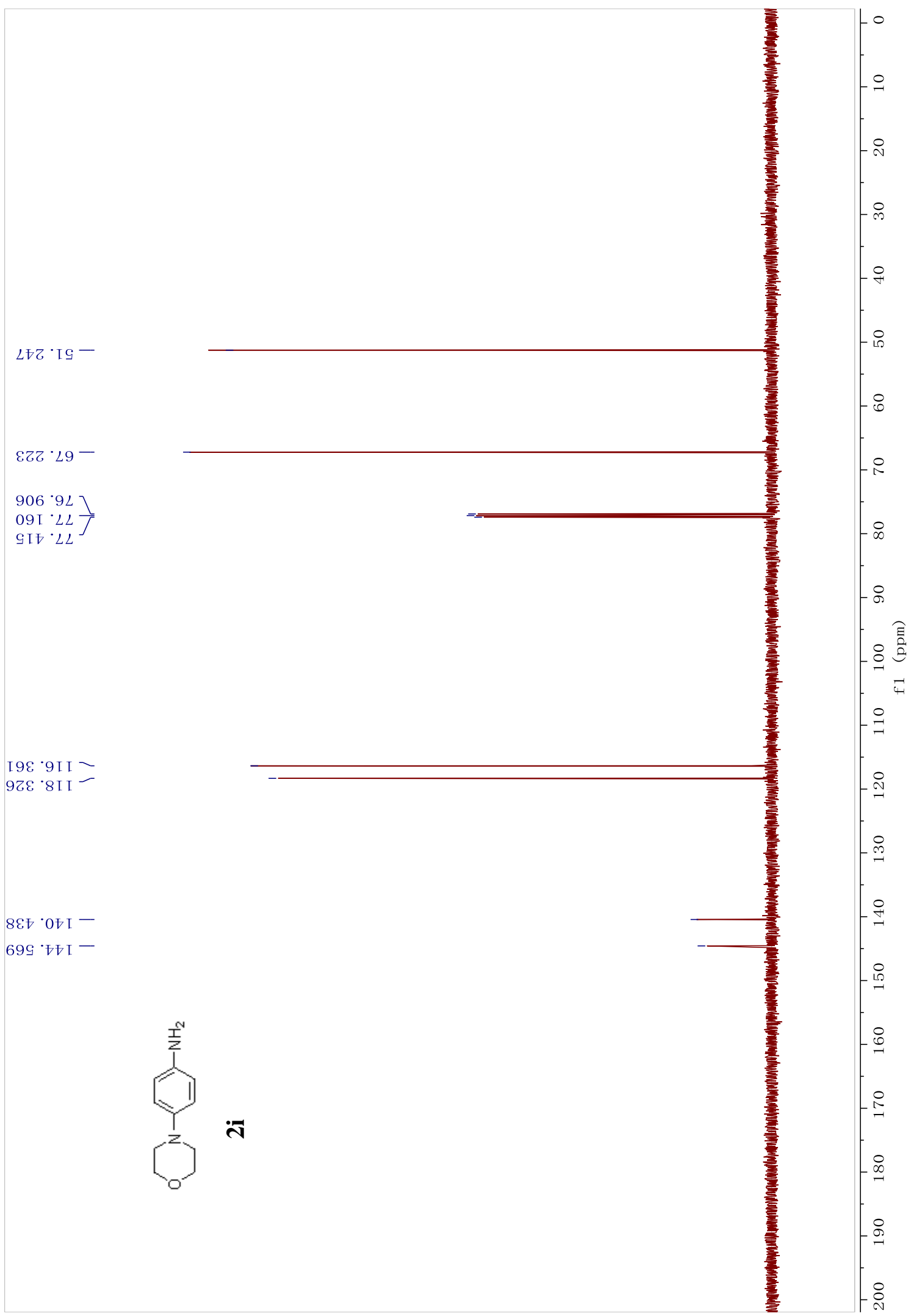




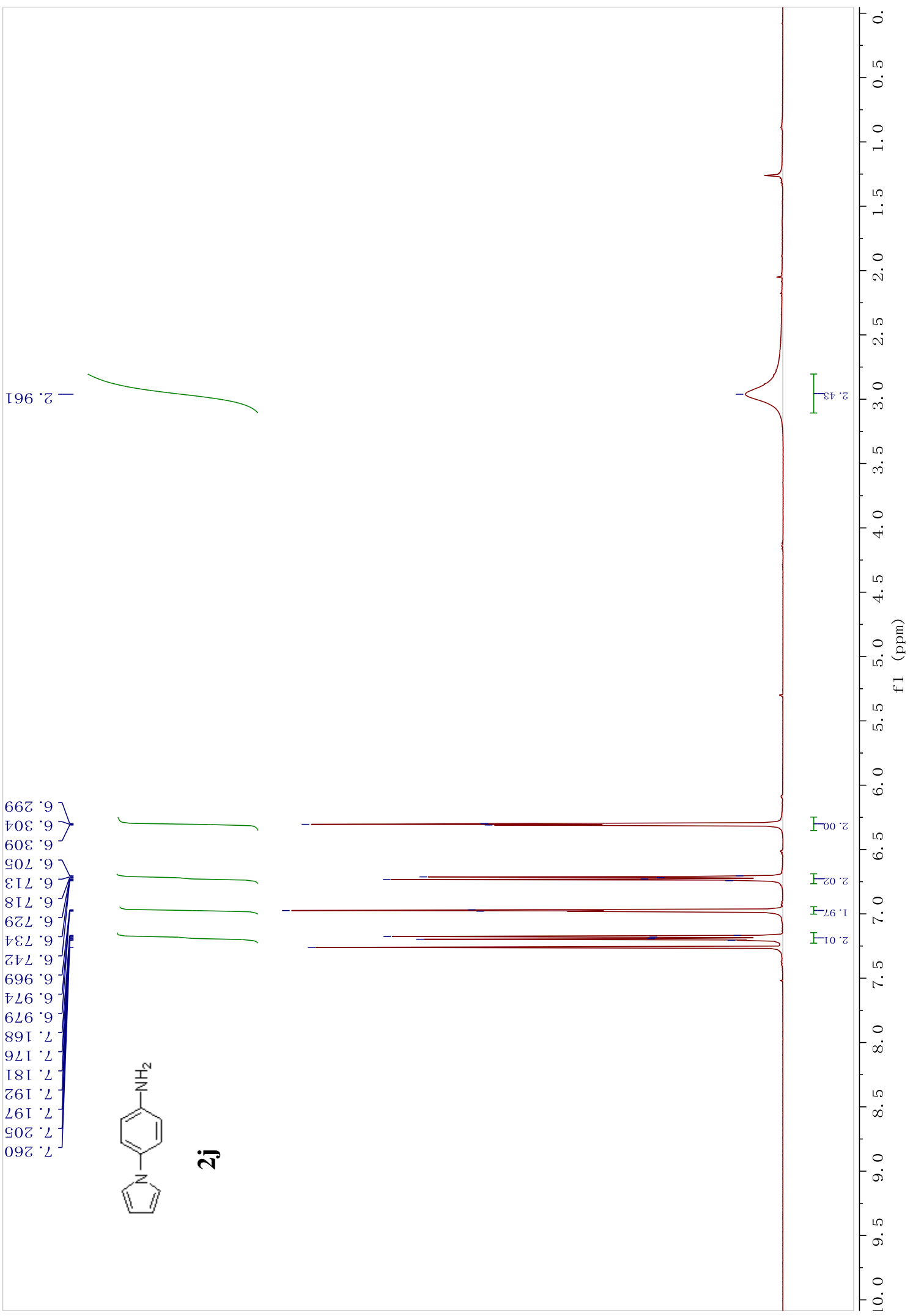


$906^{\circ} 94$

$091 \cdot 2 L J$

I99 60 I

I $8 L^{\circ}$ GI

$908^{\circ} 6$ I I

I67 $2 \mathrm{I}^{\circ} \mathrm{I}$

E†0 ЕE I -

$979^{\circ}$ tたI

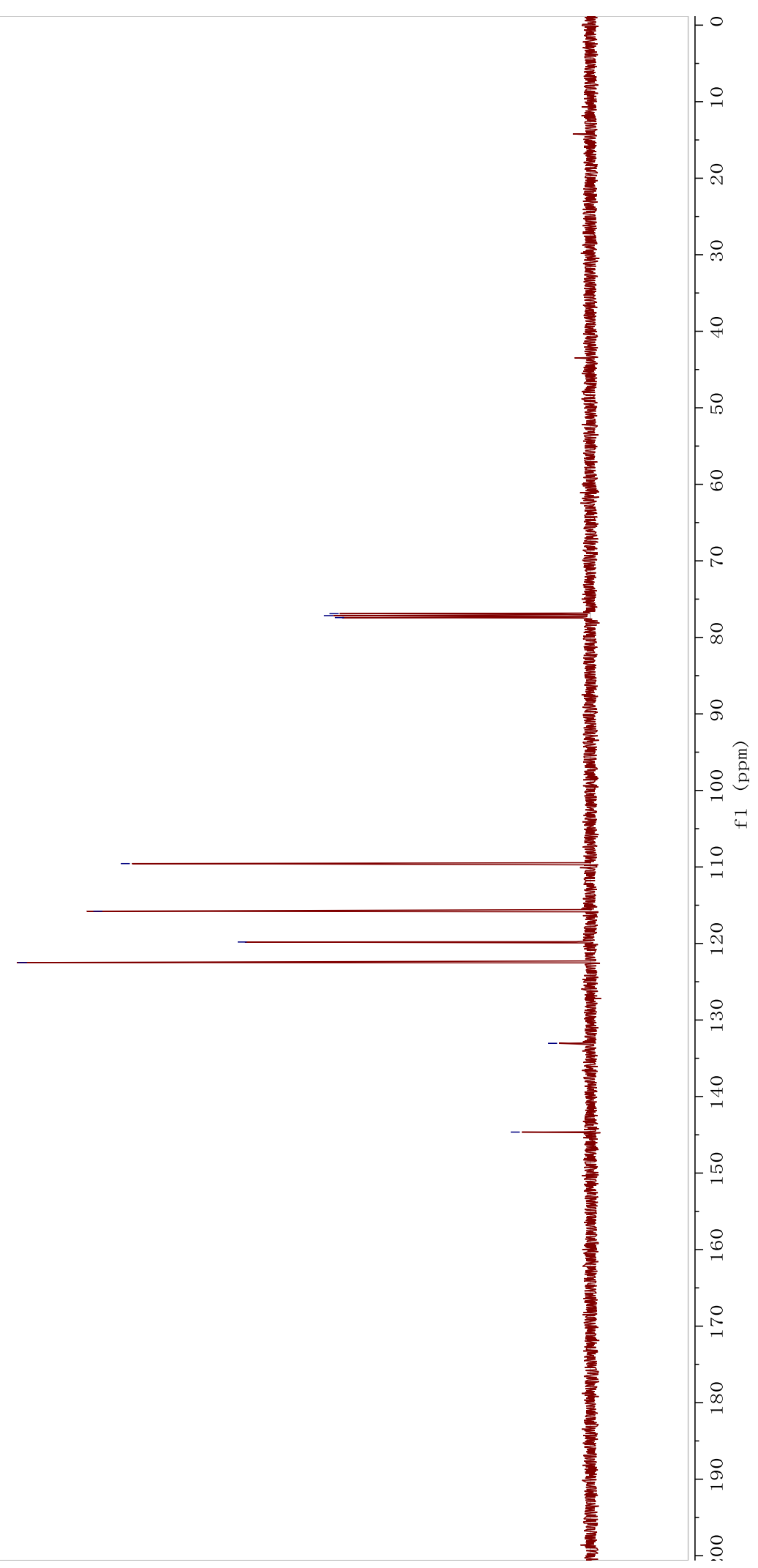




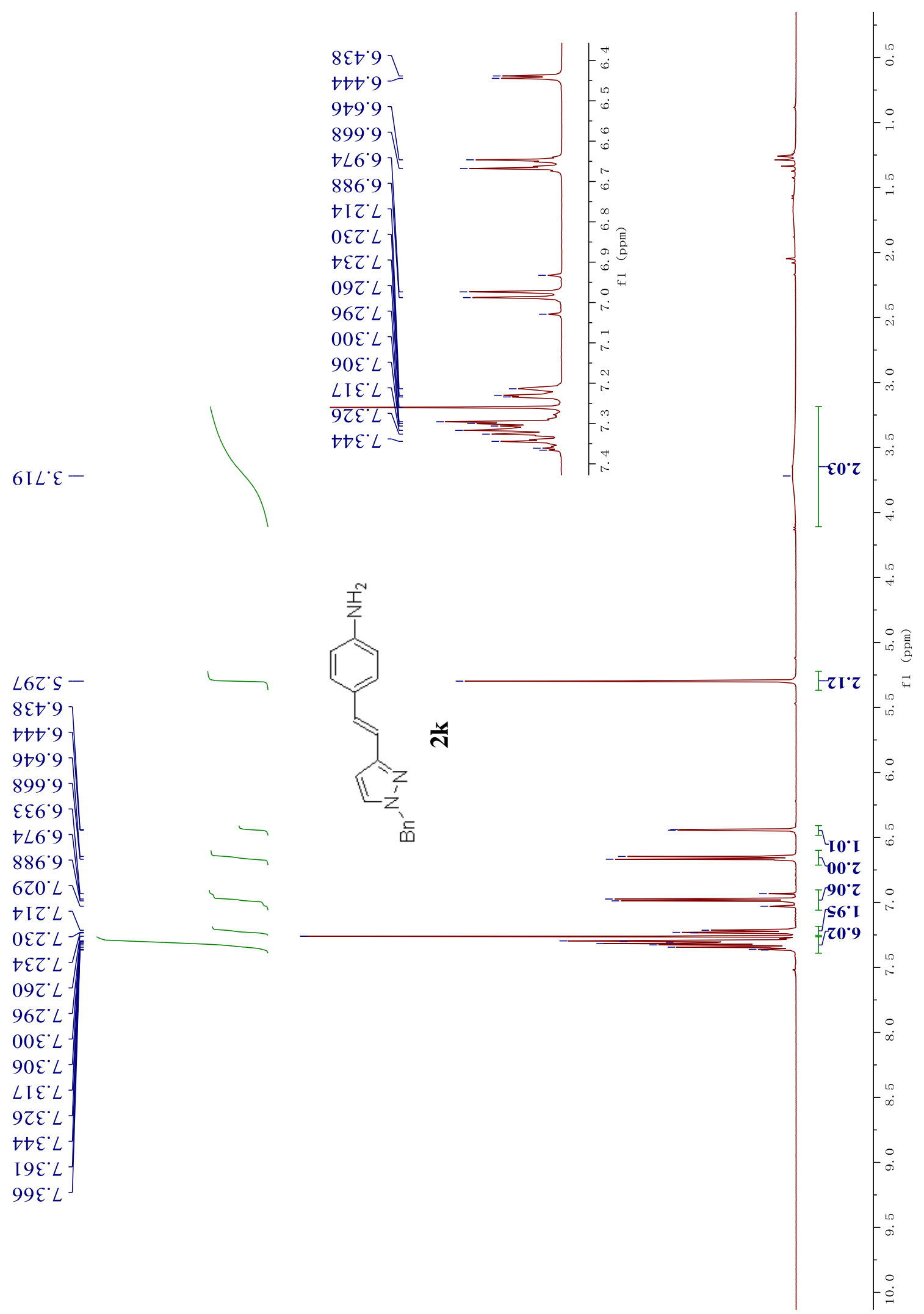




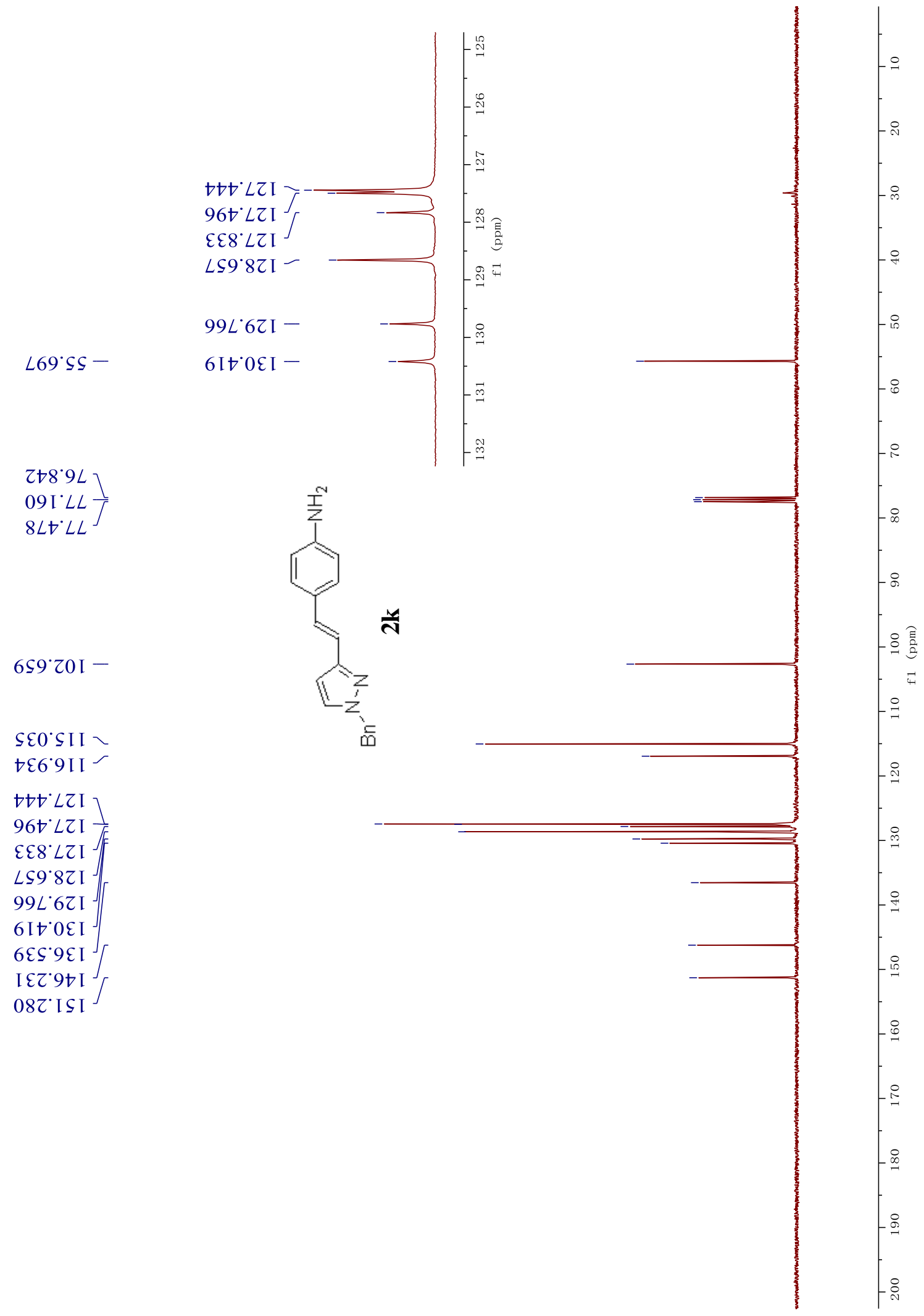




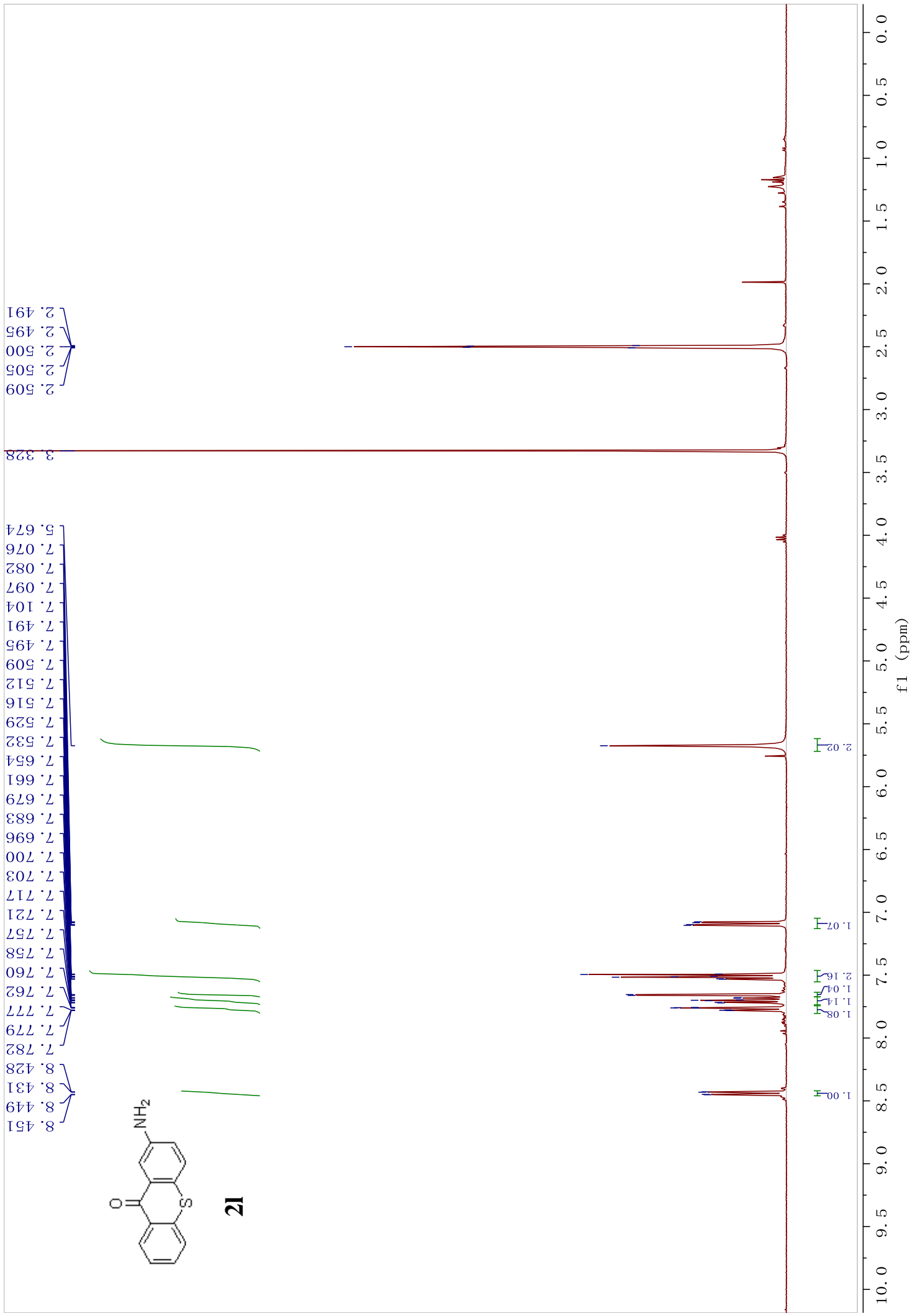



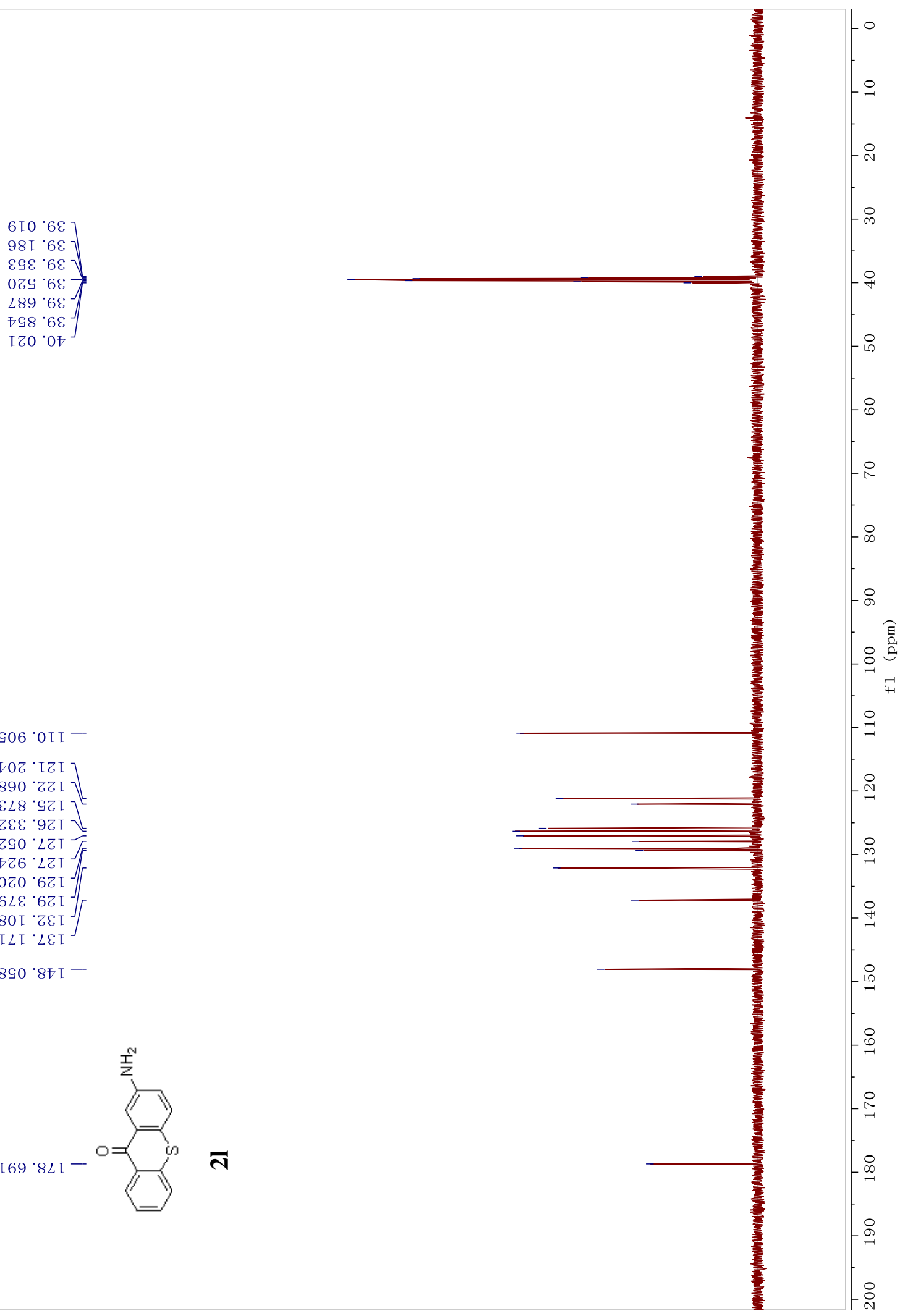

$906^{\circ}$ OI I

$70 \zeta^{\cdot}[\mathrm{L}[\mathrm{]}]$

$890^{\circ} \mathrm{ZZ}[\mathrm{I}]$

\&L8 GZ[ ]

ZEE 9 I I

ZSO LZI T

† 6 . LZI

$070 \cdot 6 Z \mathrm{I}^{-}$

$6 L \varepsilon \cdot 6 Z \mathrm{I}$

$80[\cdot \zeta \&[$

I $L \mathrm{I} \cdot \mathcal{E} \mathrm{E}$

$890 \cdot 87 \mathrm{I}-$

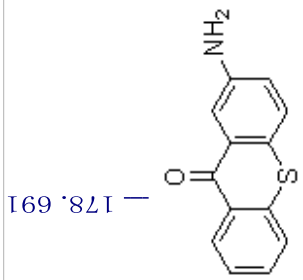


$\downarrow t L^{\circ} \varepsilon-$

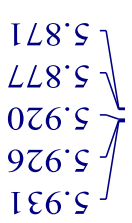

I9Z'L-<smiles>COc1cc([14CH2])cc(OC)c1</smiles>

\section{งี}
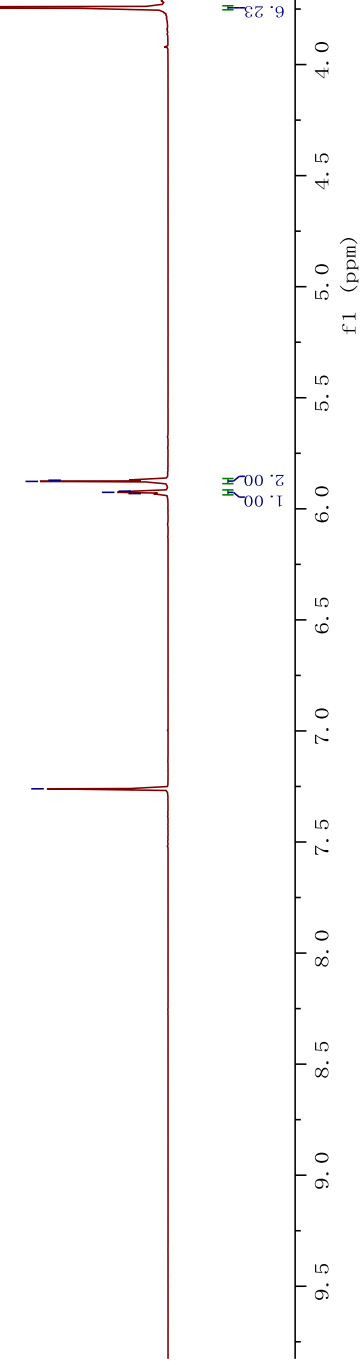
$2 Z 0^{\circ} \varsigma$ -

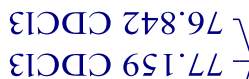

EIगด 08+ $L L$

69L.06 $\angle 29^{\circ} \varepsilon 6^{-}$

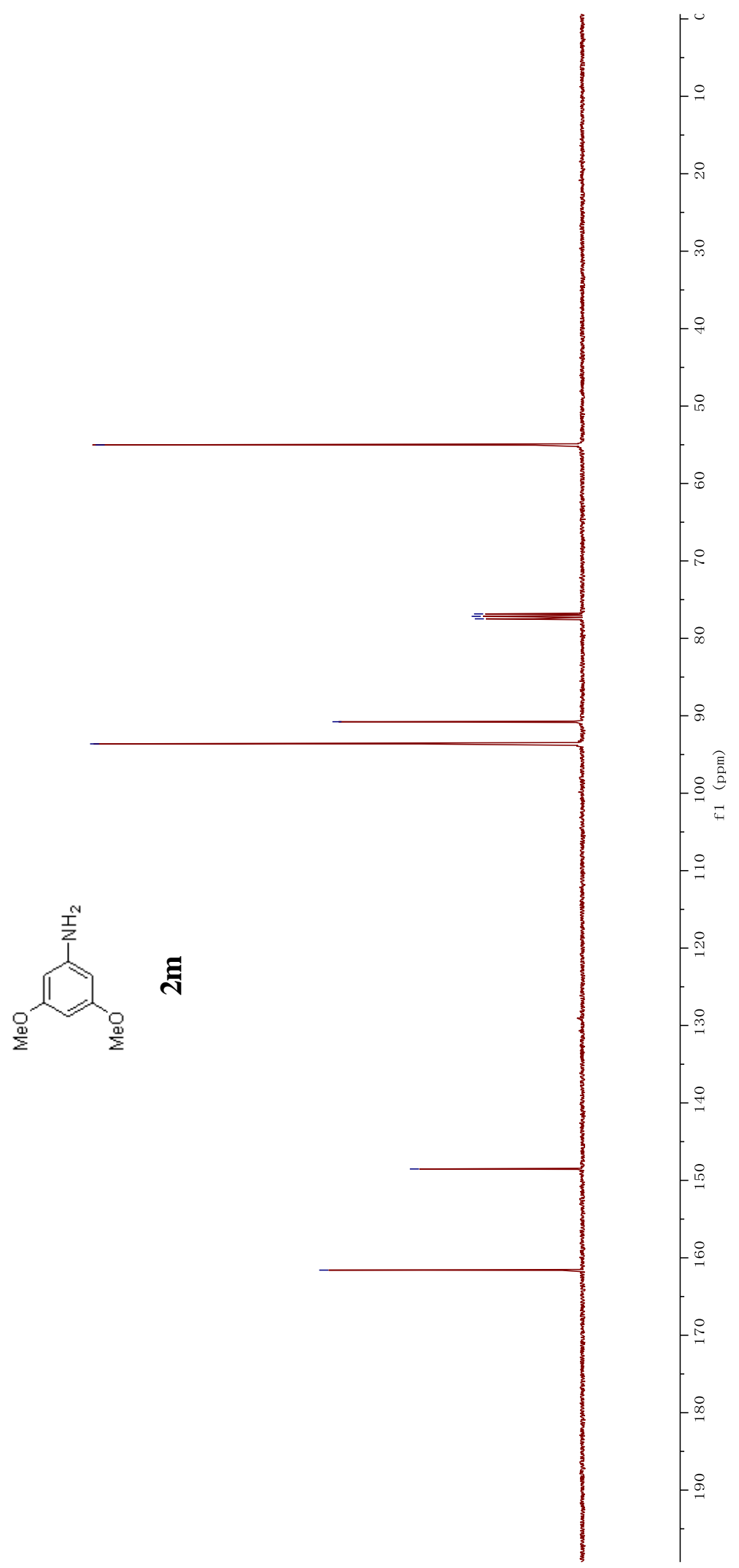

$8 Z \varsigma^{\circ} 8 \mathrm{tI}-$

26S. I9I -

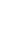




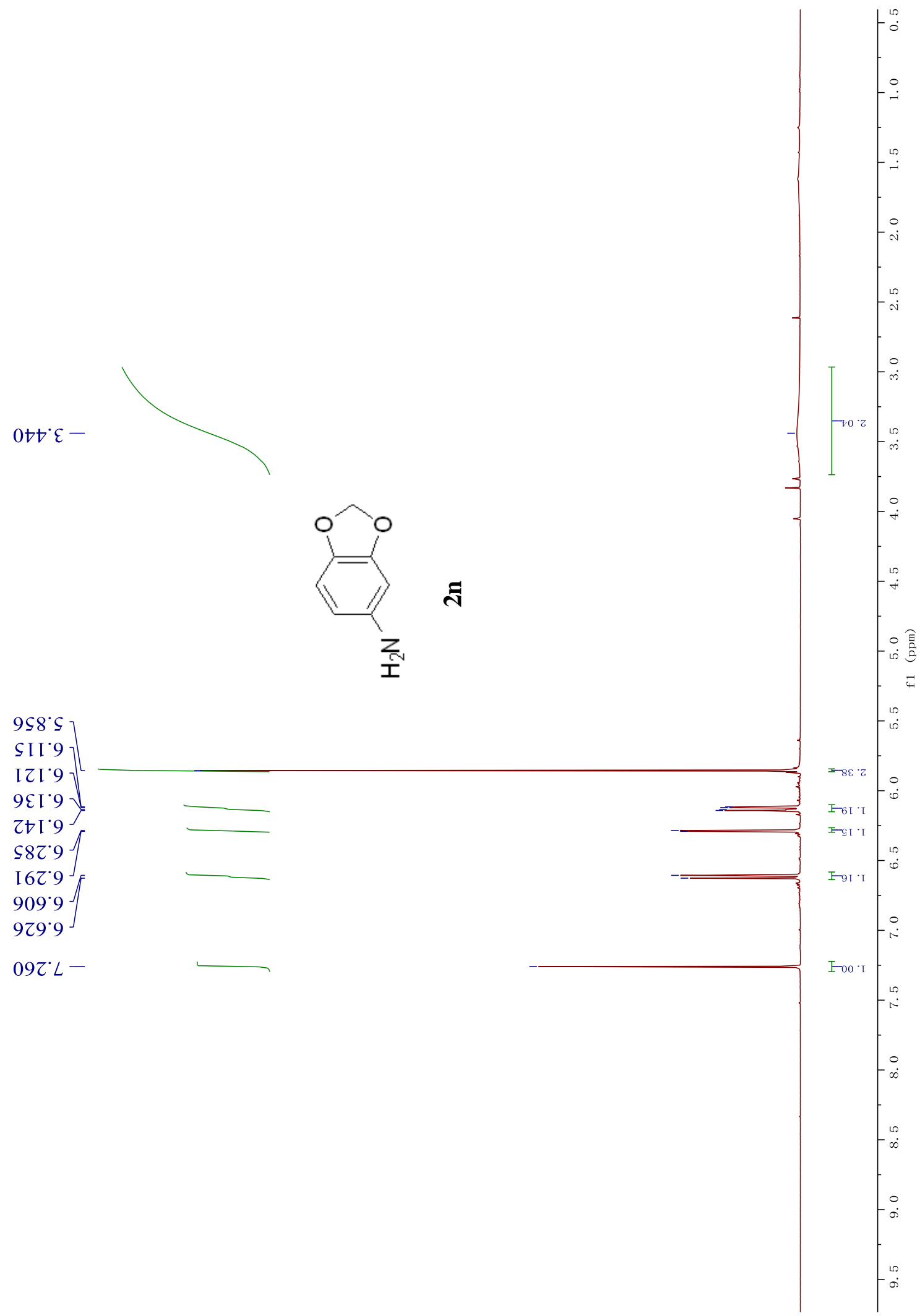




$$
\left.\begin{array}{l}
Z \sqcup 8^{\circ} 9 L \\
6 S I^{\circ} L L I \\
8 L t^{\circ} L L
\end{array}\right]
$$

$080.86-$ Z\$9.00 I S88.90I ᄂ $8 L S^{\circ} 80 I^{-}$

$662^{\circ} 0 t \mathrm{I}$ 6St It I $08 I^{\circ} 8 \mathrm{t} \mathrm{I}-$

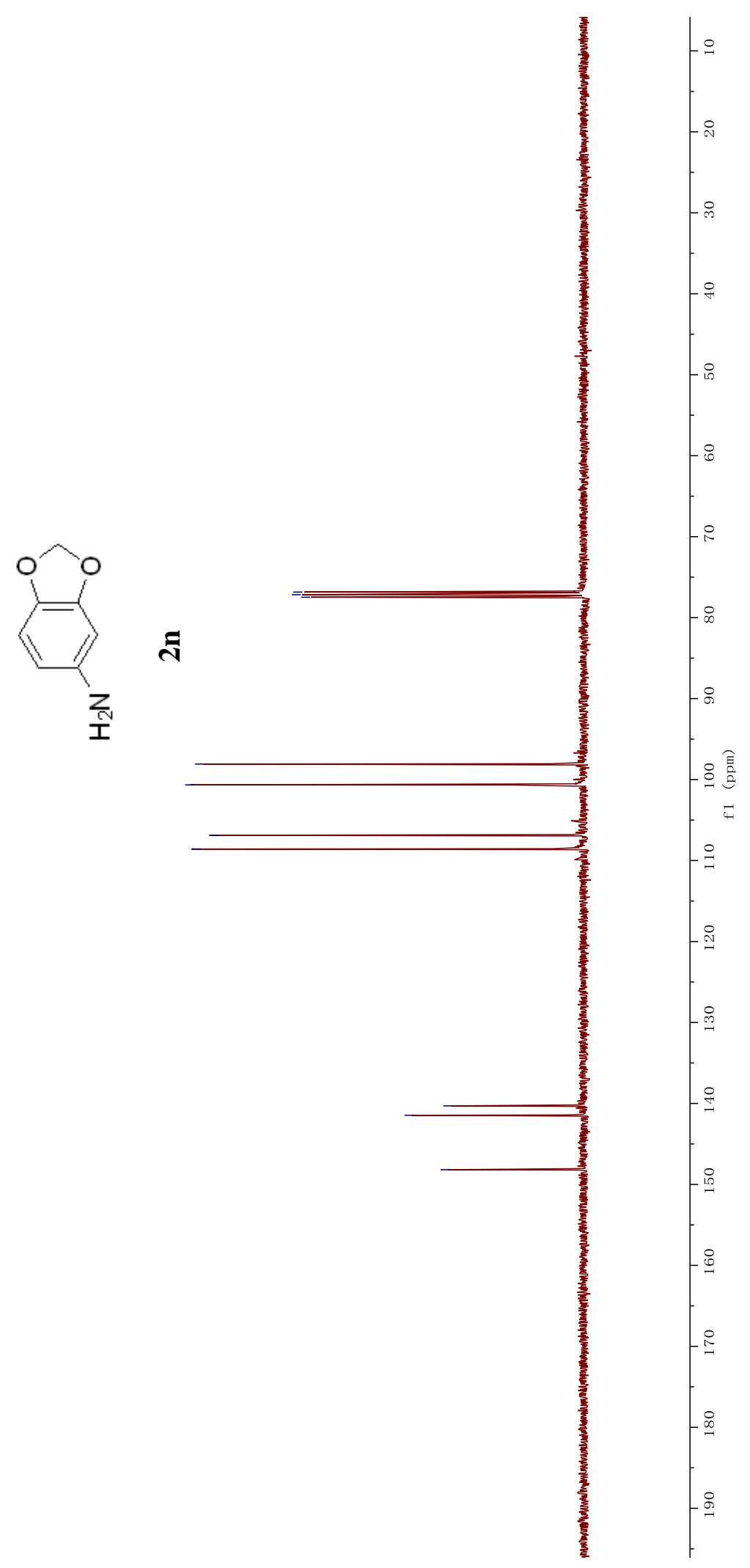




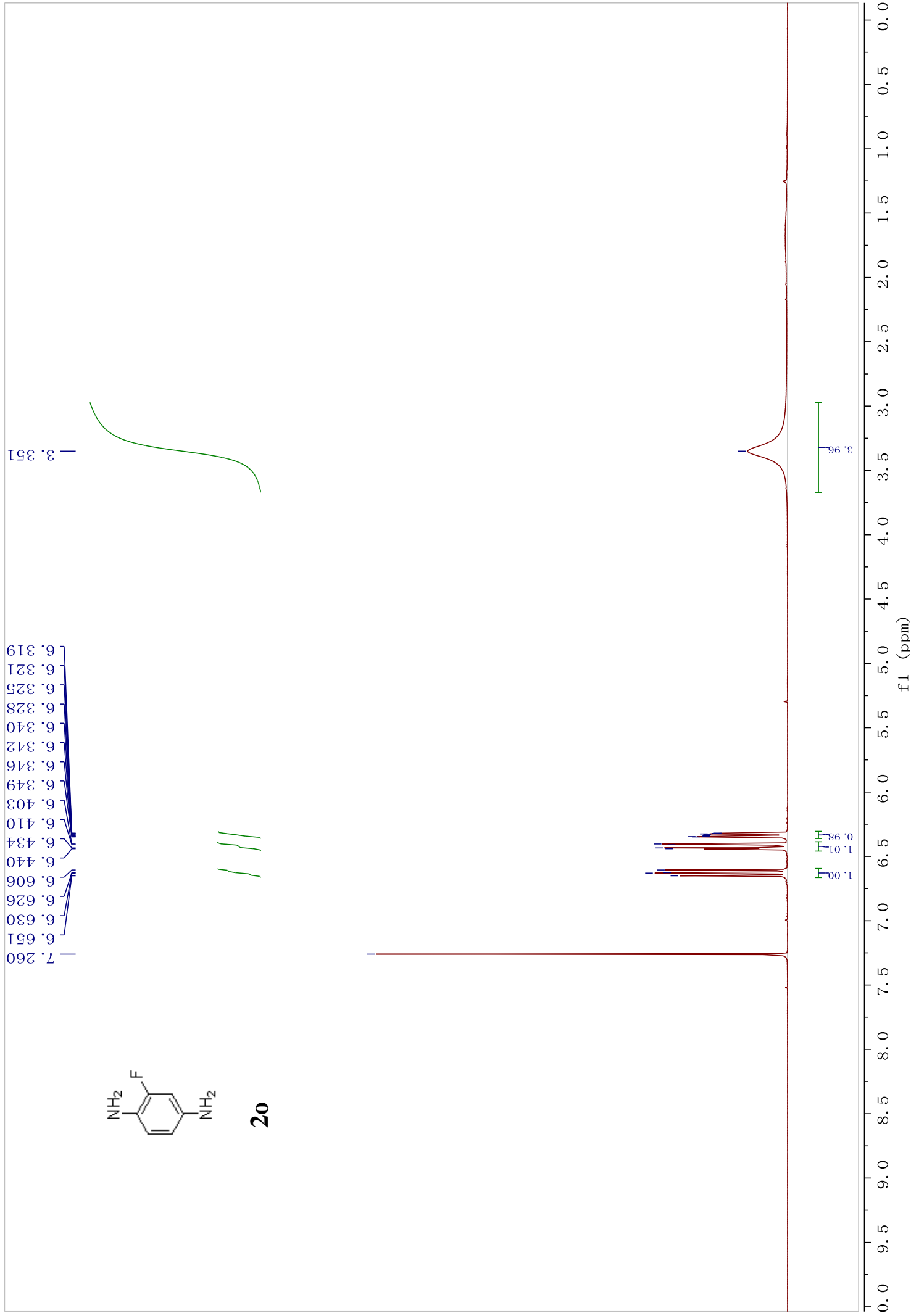


$906 \cdot 9 L$
$091 \cdot 2 L$
$\emptyset[t \cdot L L$

$\angle \& G \cdot 80 \mathrm{I}\rangle$

IIL $E$ I I

$\angle \mathrm{Eg} \cdot \operatorname{IIL}\rangle$

ELG'III

$8 \& 9^{\circ} 8 \mathrm{II}>$

$949 \cdot 8$ I I

I66.9ZI

$660 \cdot 97 \mathrm{I}$

† $\left.9 \varepsilon^{\circ} 6 \varepsilon \mathrm{I}\right\rangle$

6马T $6 \varepsilon$ I

$\varepsilon \mp 9 \cdot \operatorname{IgI} \sim$

$9 \varepsilon \mathrm{G} \cdot \varepsilon \mathrm{I}$ I

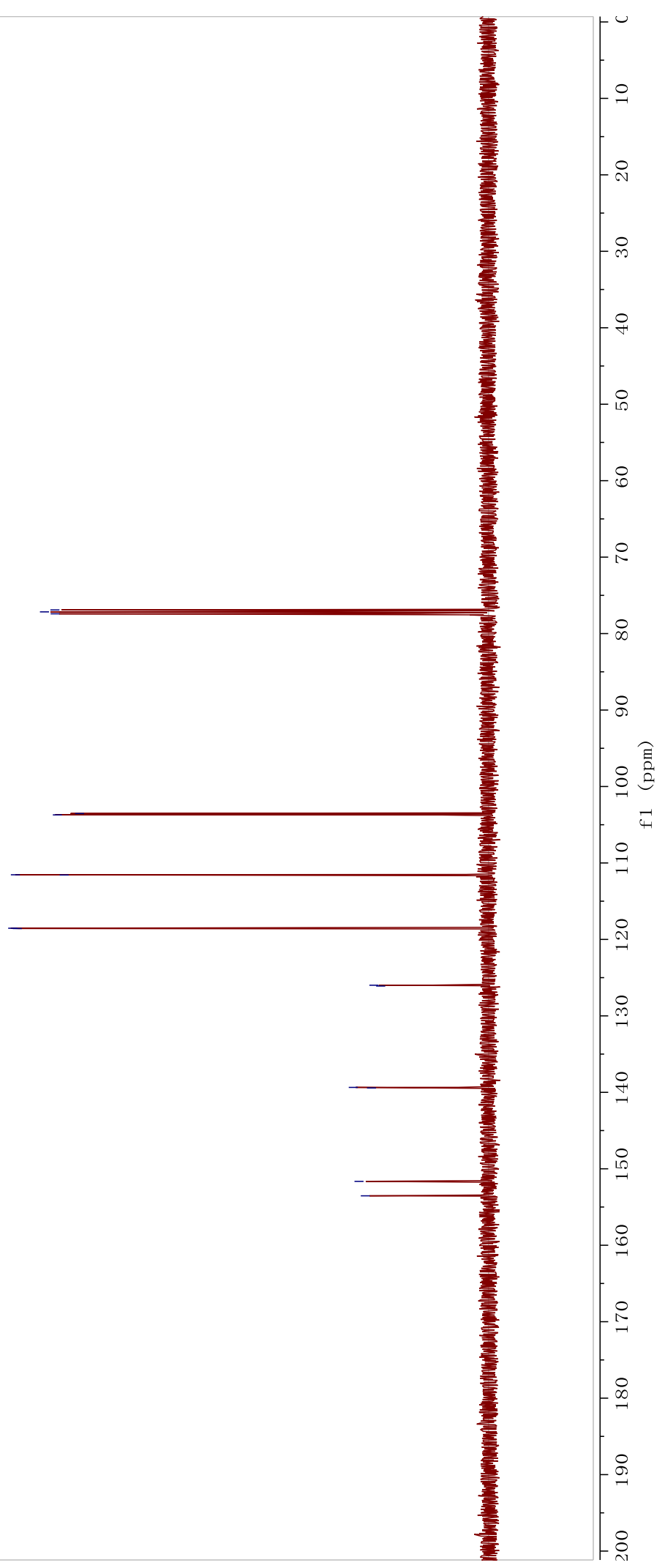




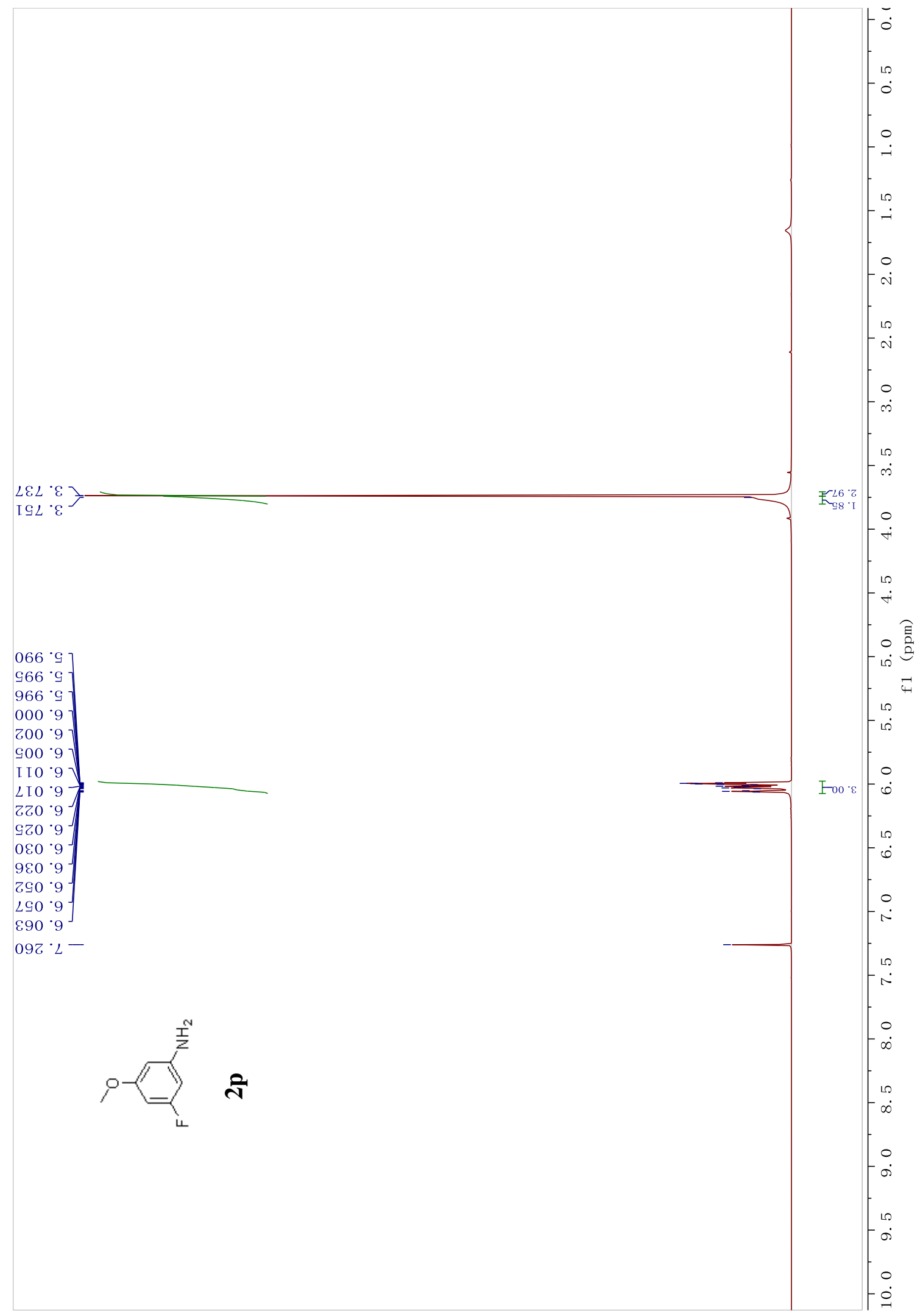




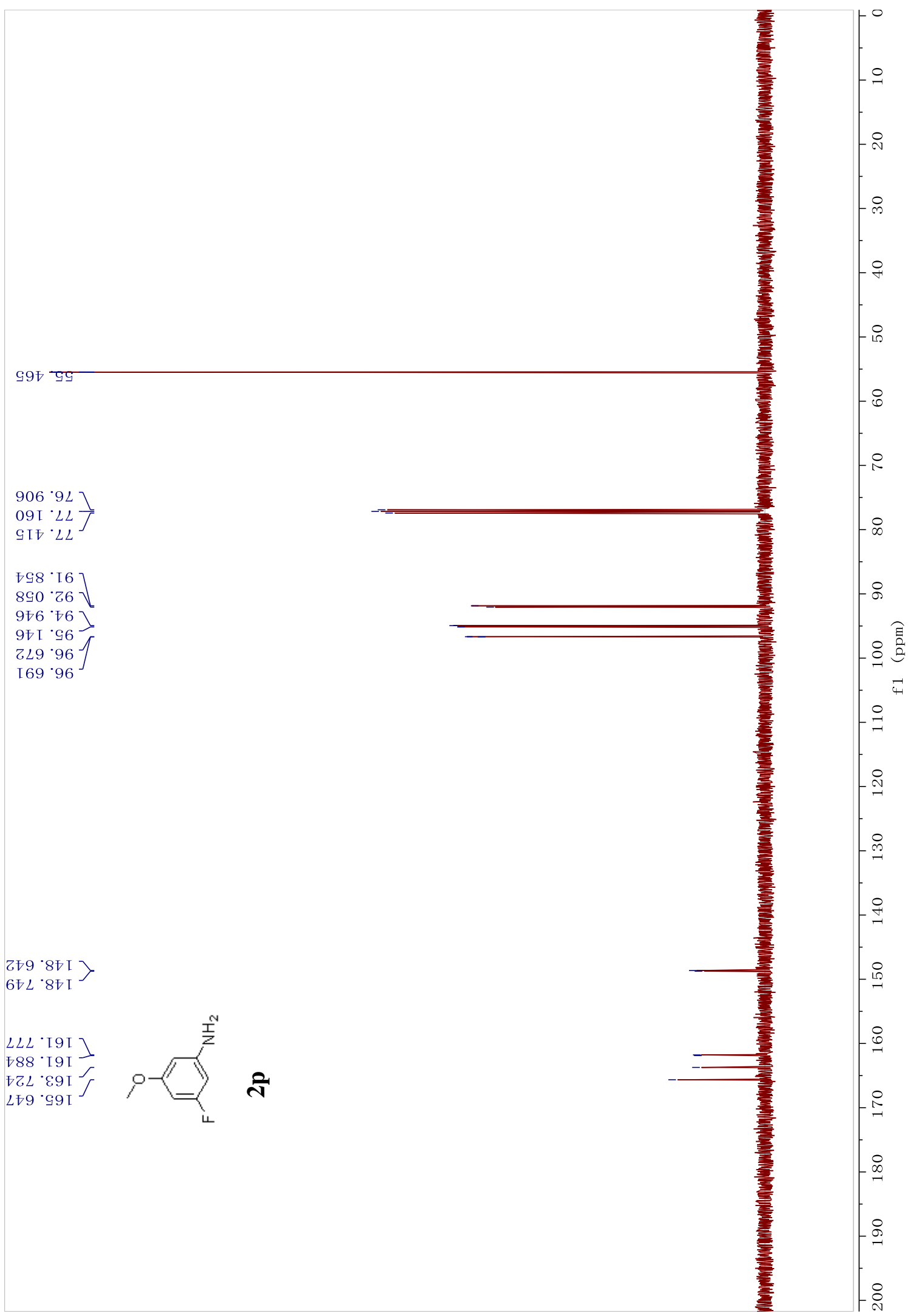




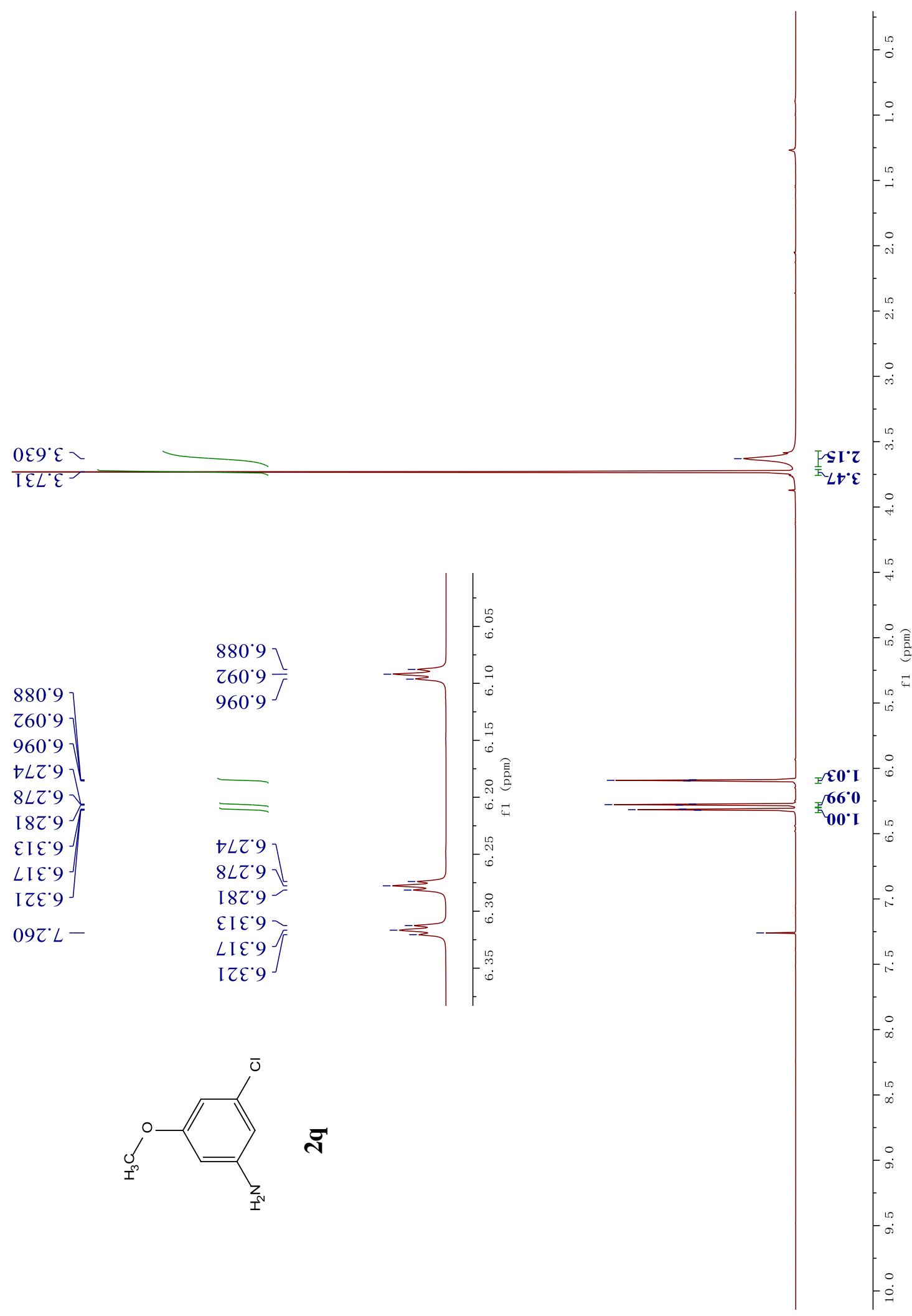




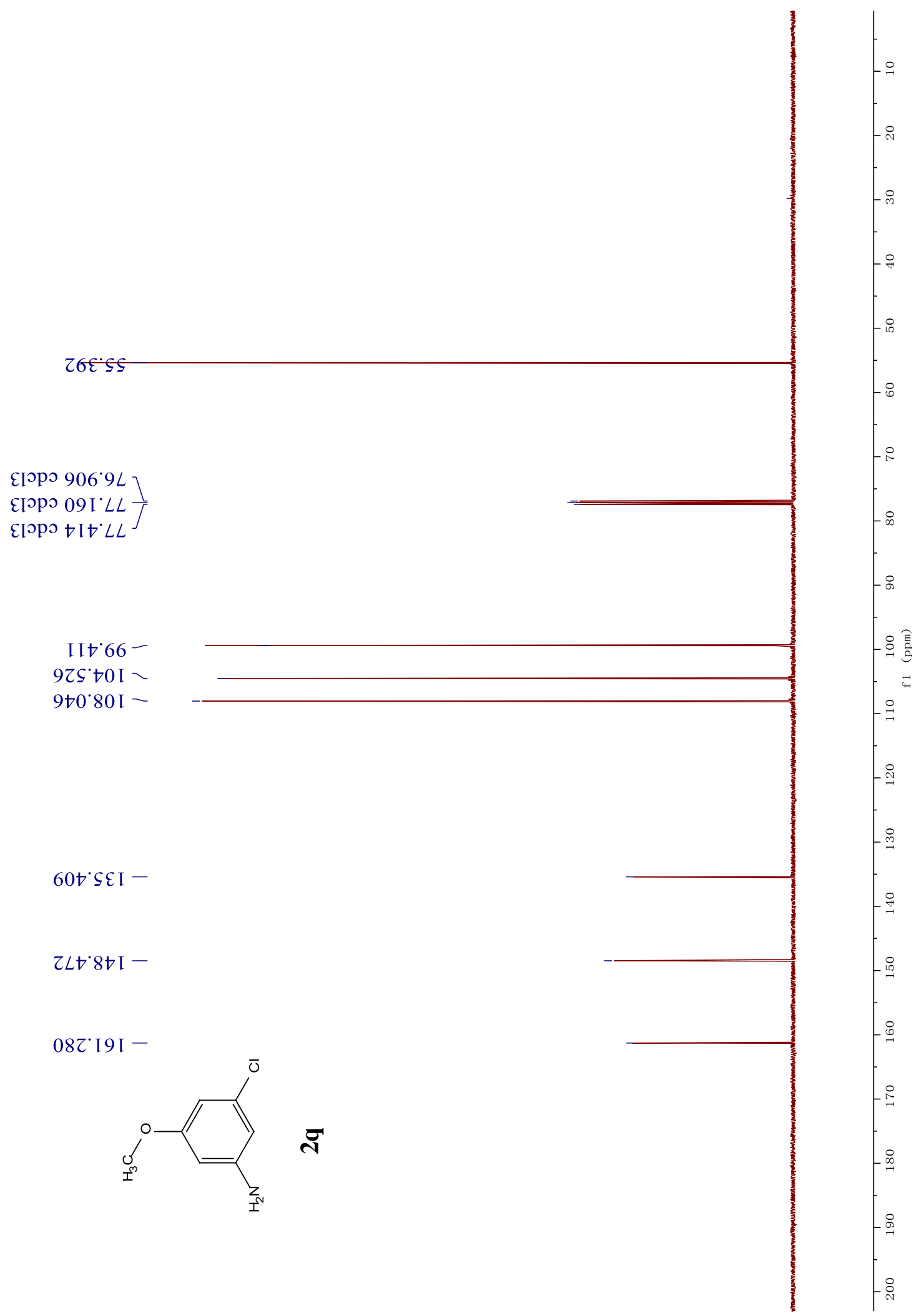




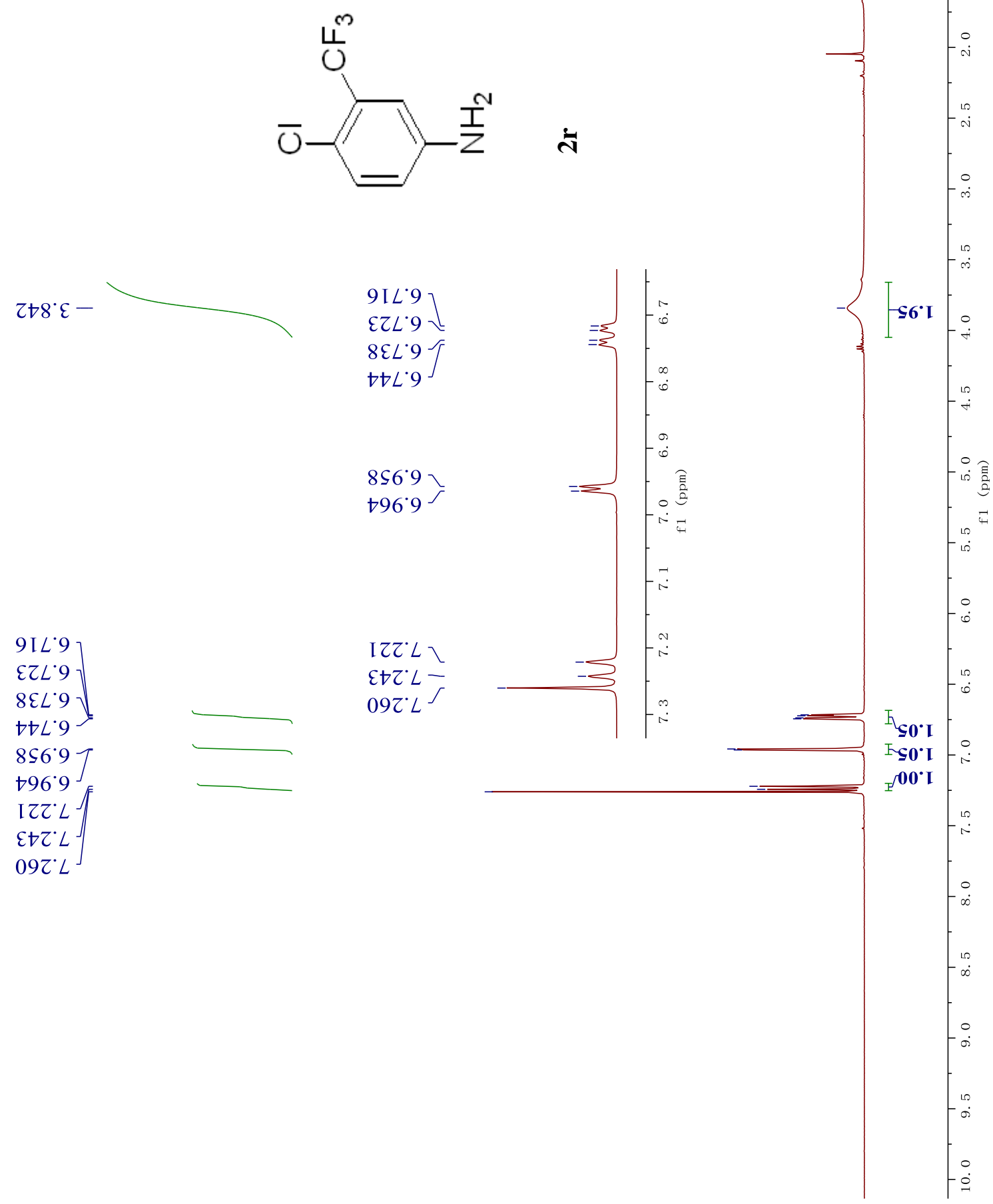




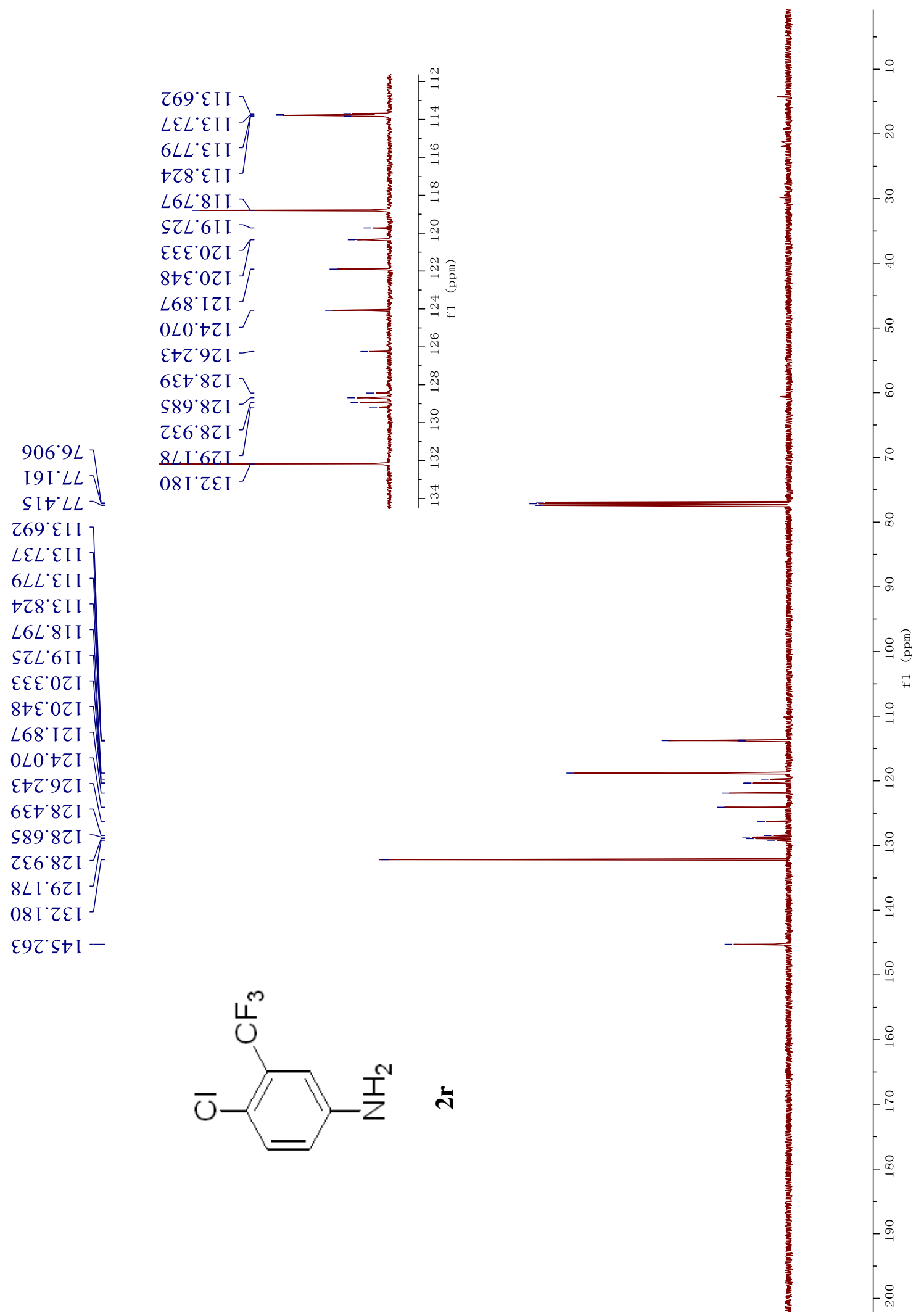




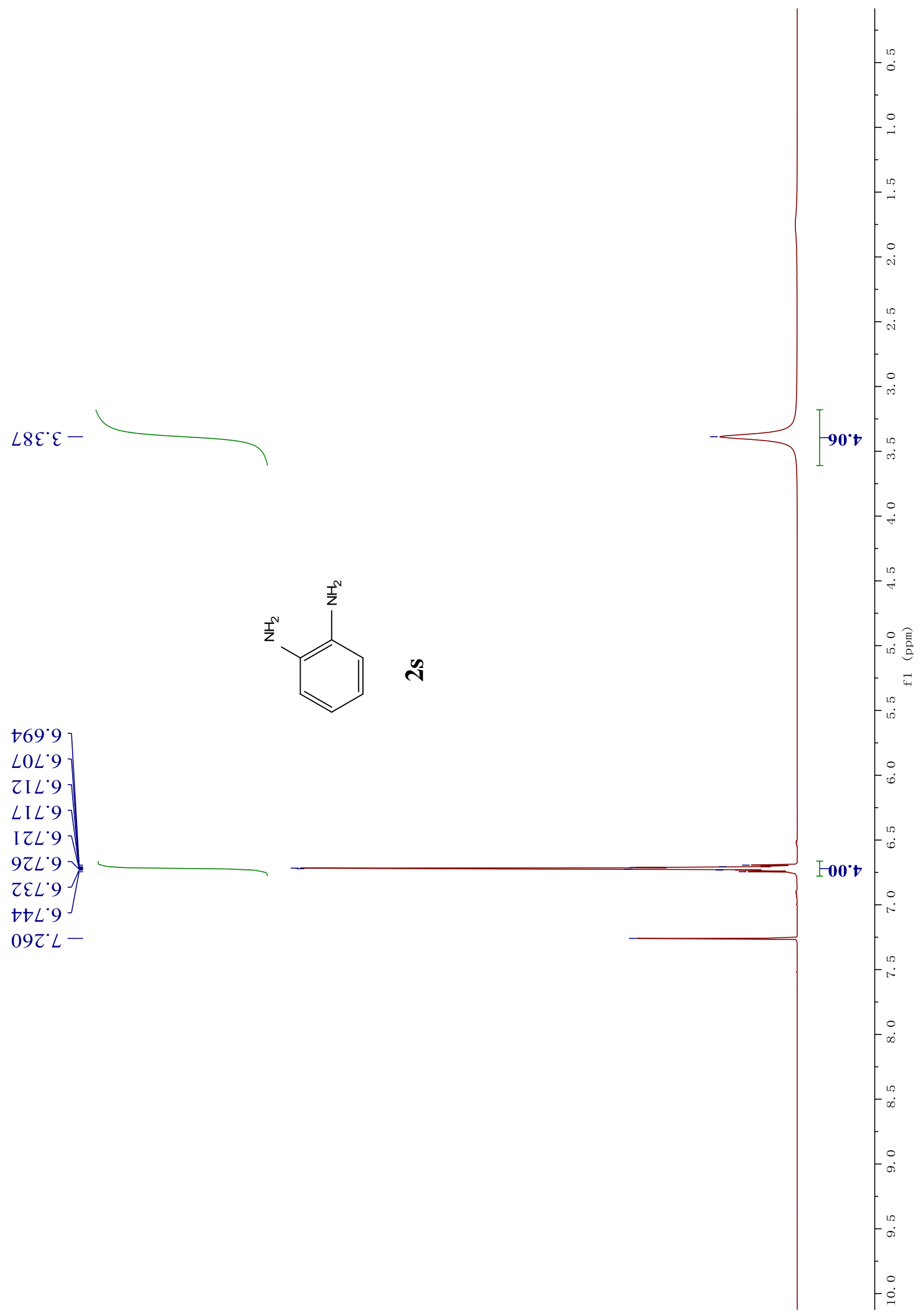



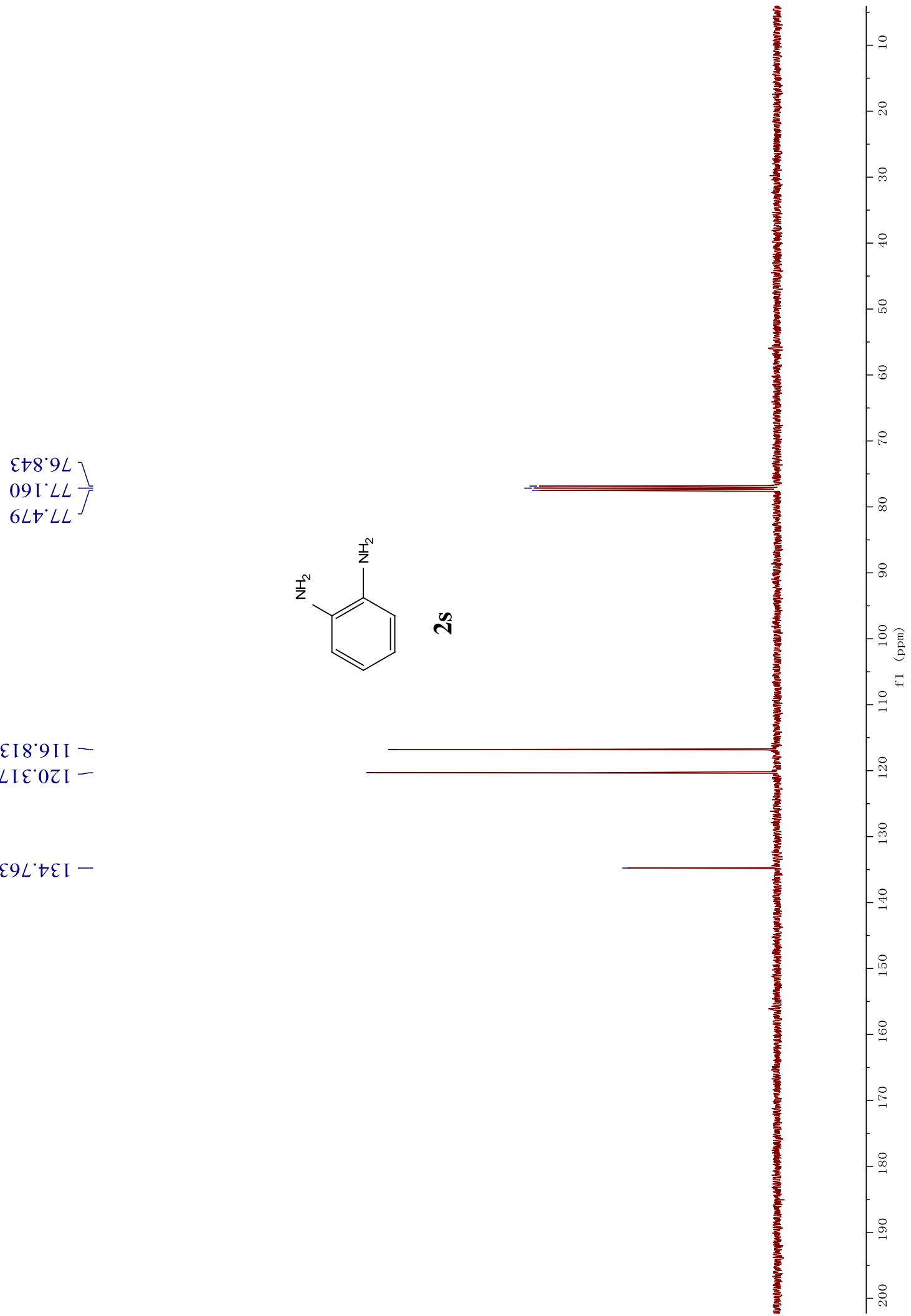

EI $8^{\circ} 9$ II -

LIEOZI -

$\varepsilon 9 L^{\circ} \nabla \varepsilon I-$ 


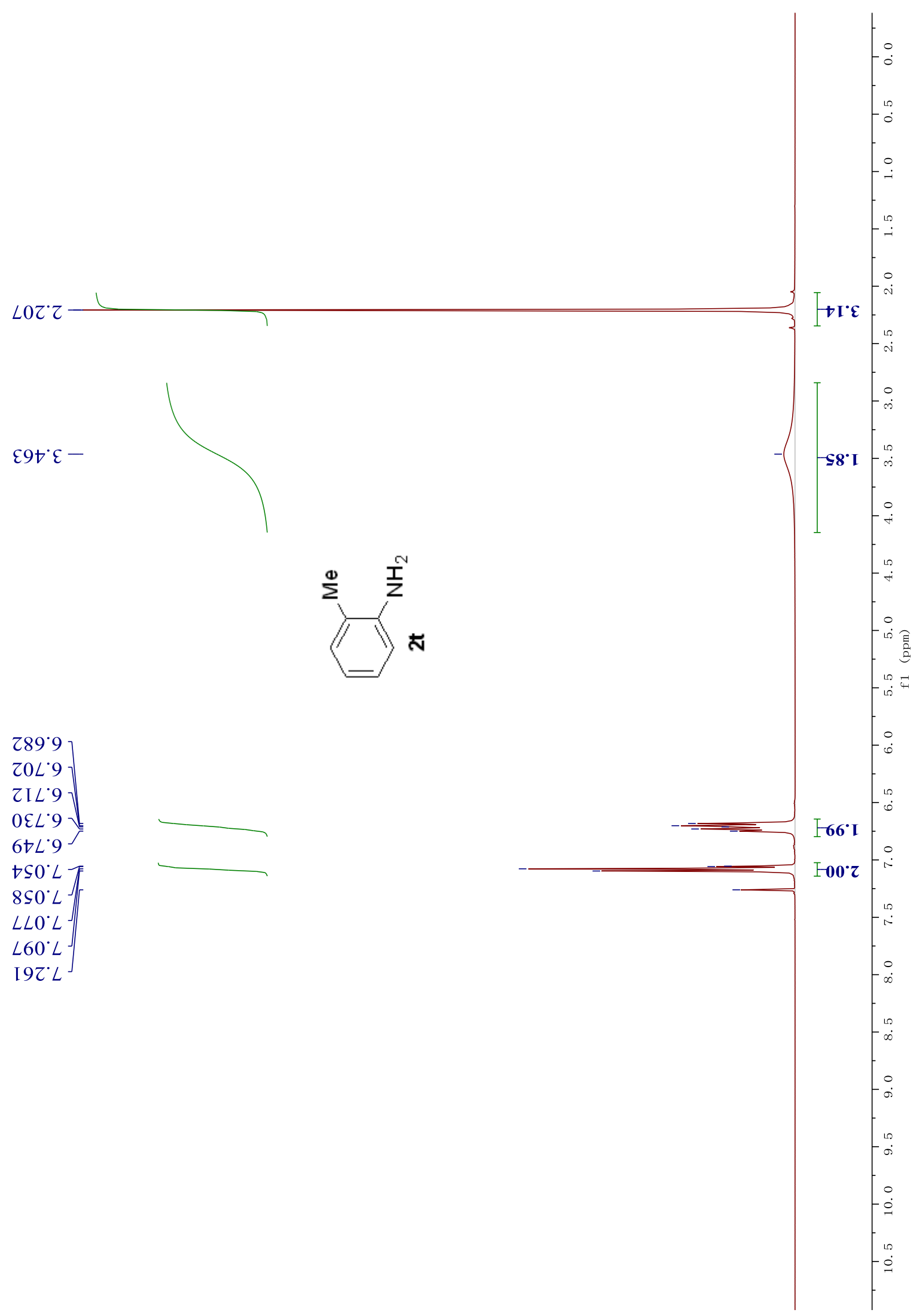


$8 L E^{\circ} L I-$

$2 \succcurlyeq 8^{\circ} 9 L$

09 [ $L L \frac{1}{5}$

$8 \angle \nabla^{\circ} L L$

L66 ${ }^{\circ}$ I I -

I $\angle 9^{\circ} 8$ I I -

Z0t ZZI -

I96.9ZI -

Itt苂I -

IES`ウヤI-

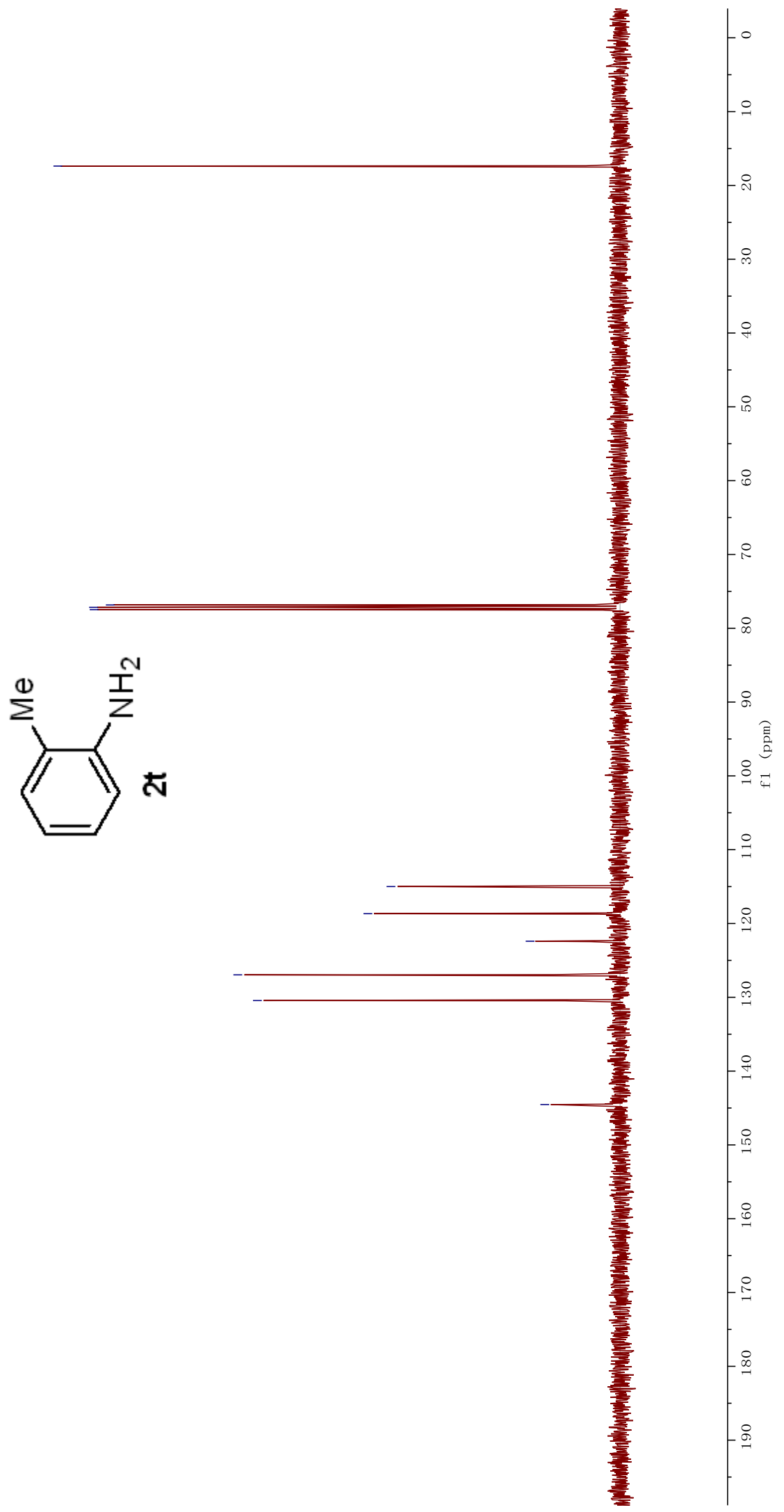


$08 I^{\circ} Z$

$\operatorname{It} L^{\circ} \varepsilon-$

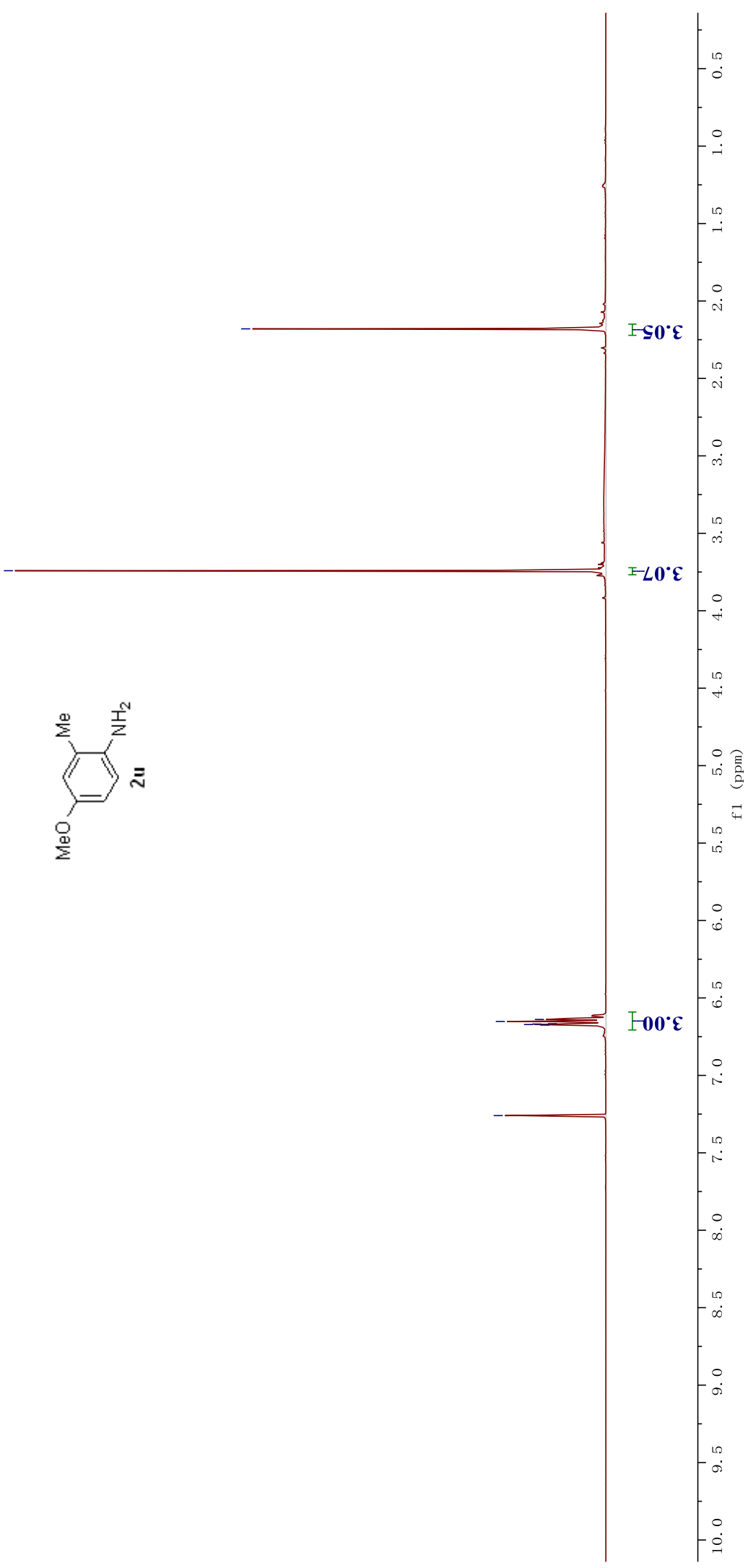

$6 \varepsilon 9^{\circ} 9$

Zऽ9.9

999.9

$899^{\circ} 9=$

ZL9.9

SL9 $9^{\circ}$

$09 Z^{\circ} L-$

i

$\stackrel{\circ}{\circ}$ 
$\varepsilon 89^{\circ} \mathrm{LI}-$

$099^{\circ} \varsigma \varsigma-$

$Z \downarrow 8^{\circ} 9 L$

I9 I' $L L T$

$8 L t \angle L$
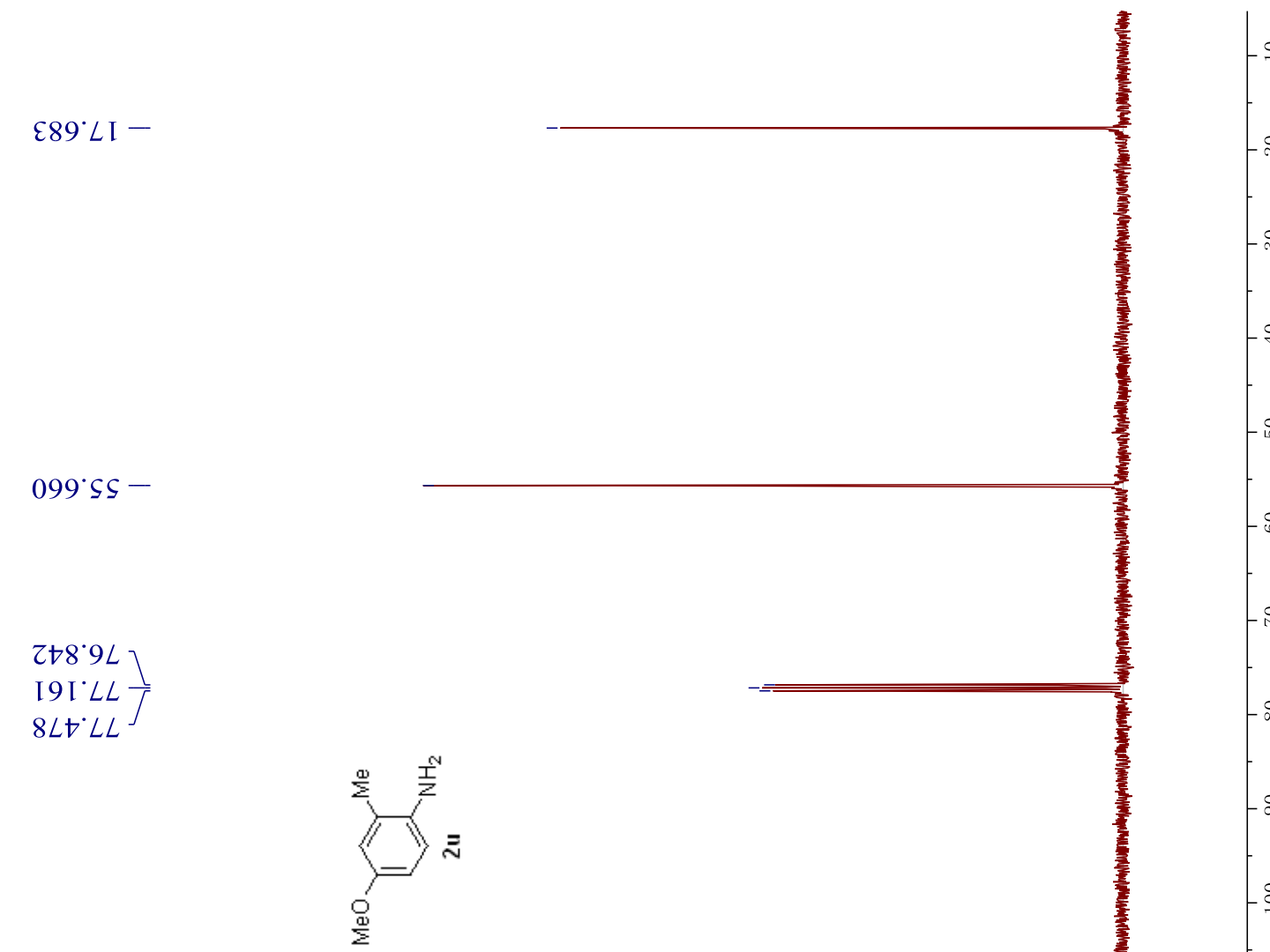

8SOZII -

LI0.9II T

8Eะ्9 I I

$\varepsilon I 0^{\circ} \triangleright Z \mathrm{I}-$

$98 I^{\circ} 8 \mathcal{E}$ I -

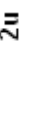

$6+9^{\circ}$ ZS I -

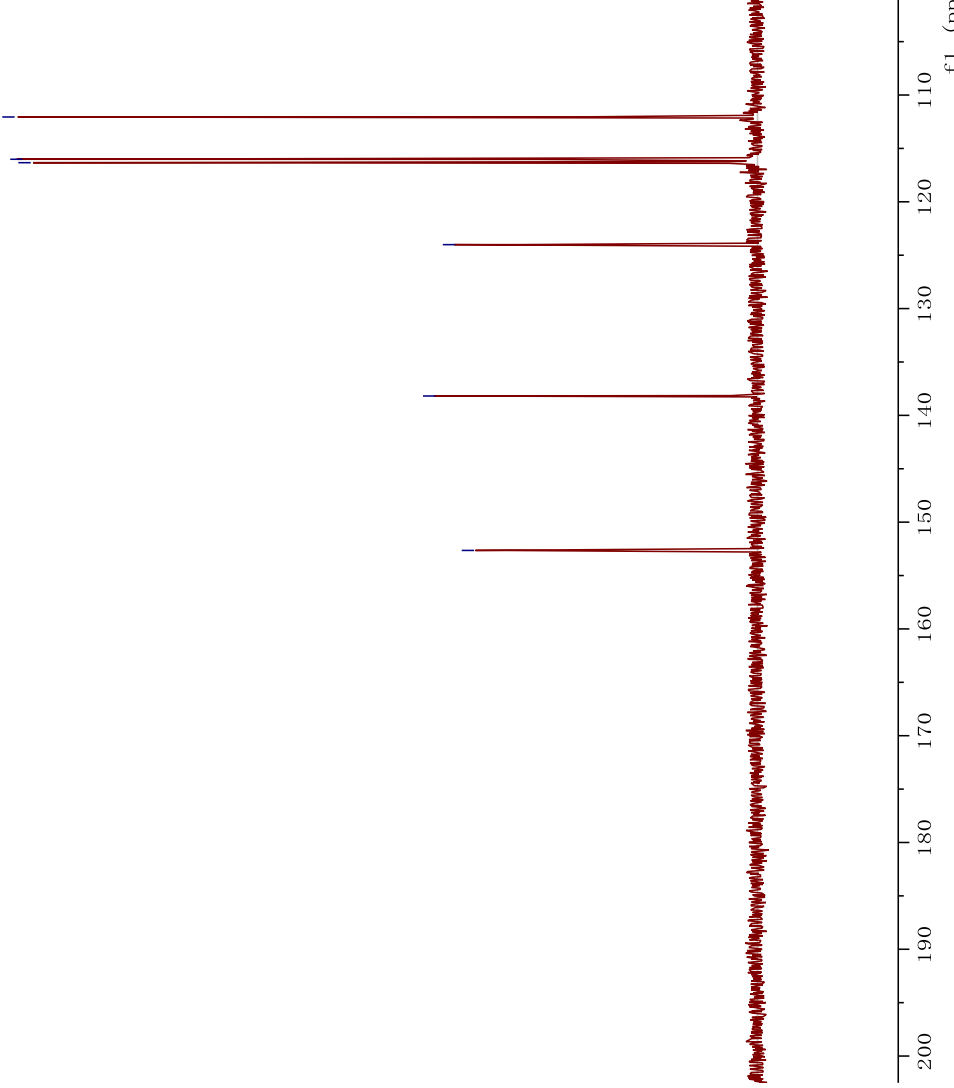


$\varepsilon \varepsilon \nabla^{\cdot} I_{-}^{-}$

IZ8 ${ }^{\circ} \varepsilon-$

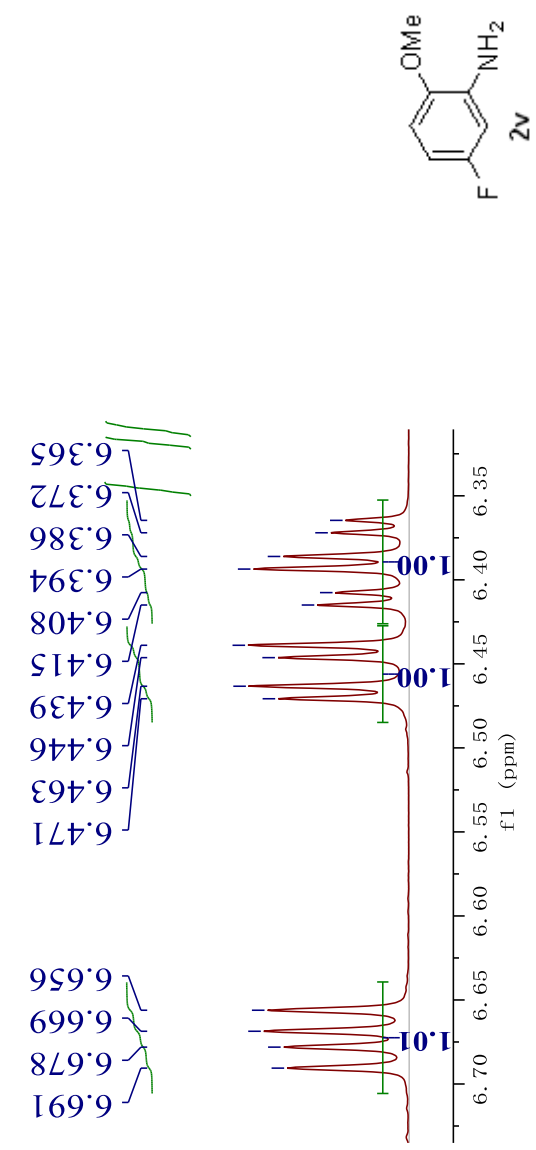

$80 t^{\circ} 9$

SIt' 9

$6 \varepsilon t^{\circ} 9$

$9 \triangleright t^{\circ} 9$

E9t' 9

I $\angle \nabla^{\circ} 9$

$959^{\circ} 9$

$699^{\circ} 9$

$8 \angle 9^{\circ} 9^{\prime}$

[69.9

$09 Z^{\circ} L$

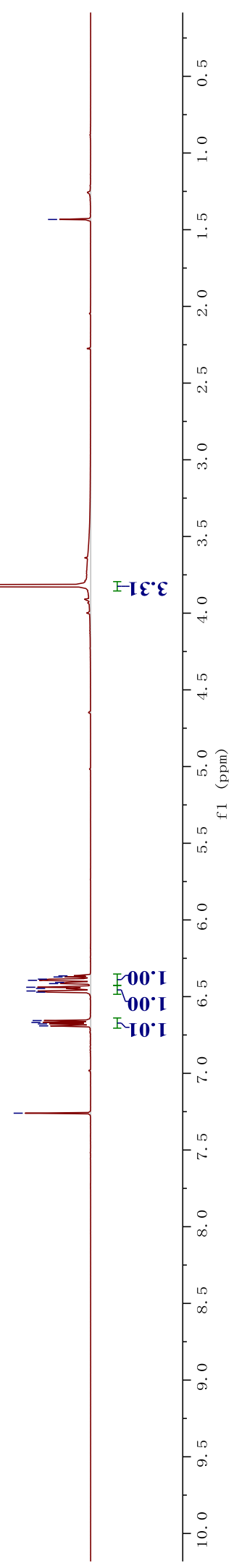



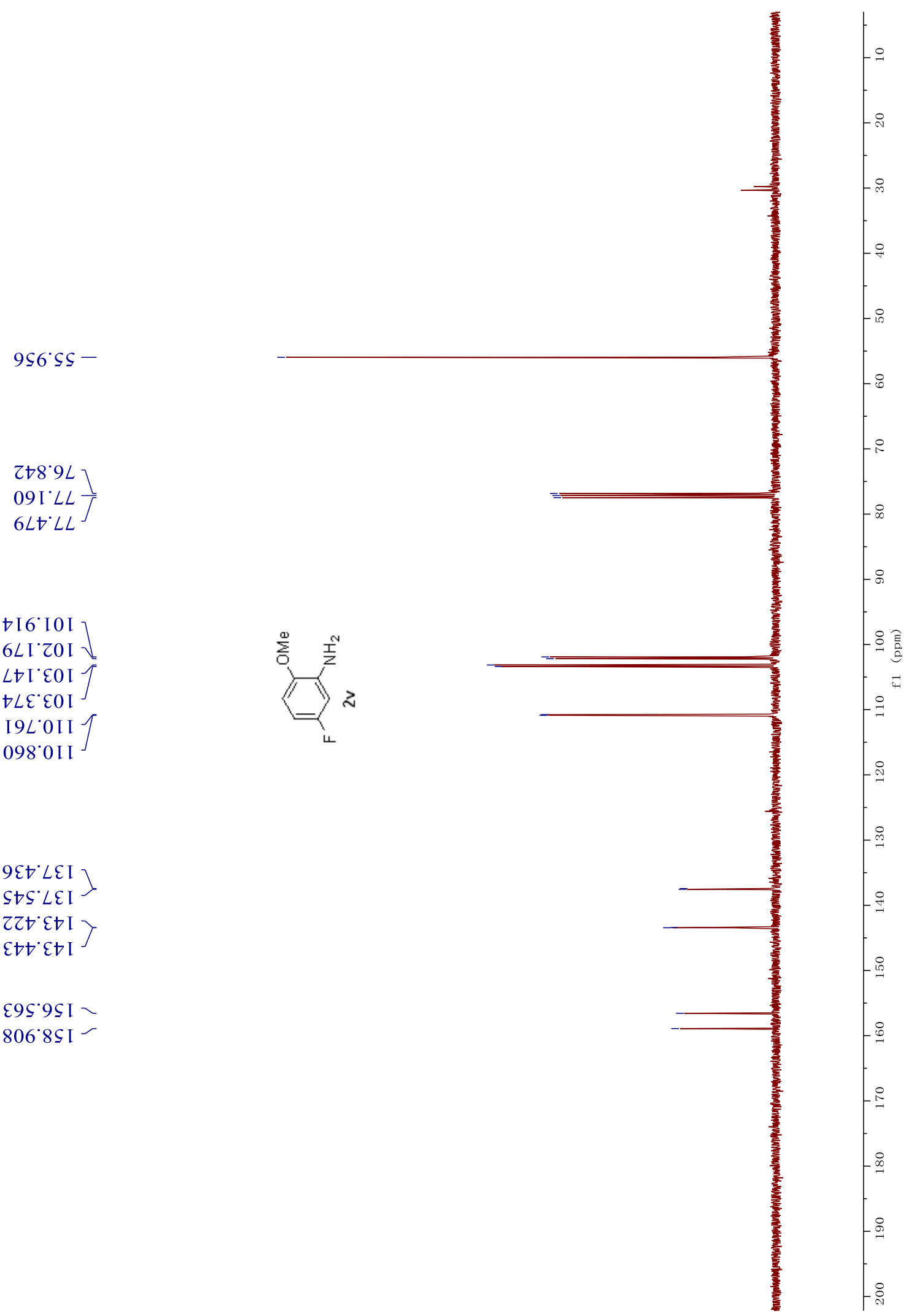

$9 \varepsilon t^{\circ} L \varepsilon \mathrm{I}$

$S \rightarrow S^{\circ} \angle E I$

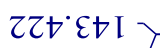

$\varepsilon \forall t^{\circ} \varepsilon+\mathrm{I} J$

E9S.9S I

$806^{\circ} 8 \mathrm{SI}^{-}$

$\stackrel{\circ}{\circ}$

$\stackrel{\circ}{\exists}$

요

은

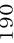




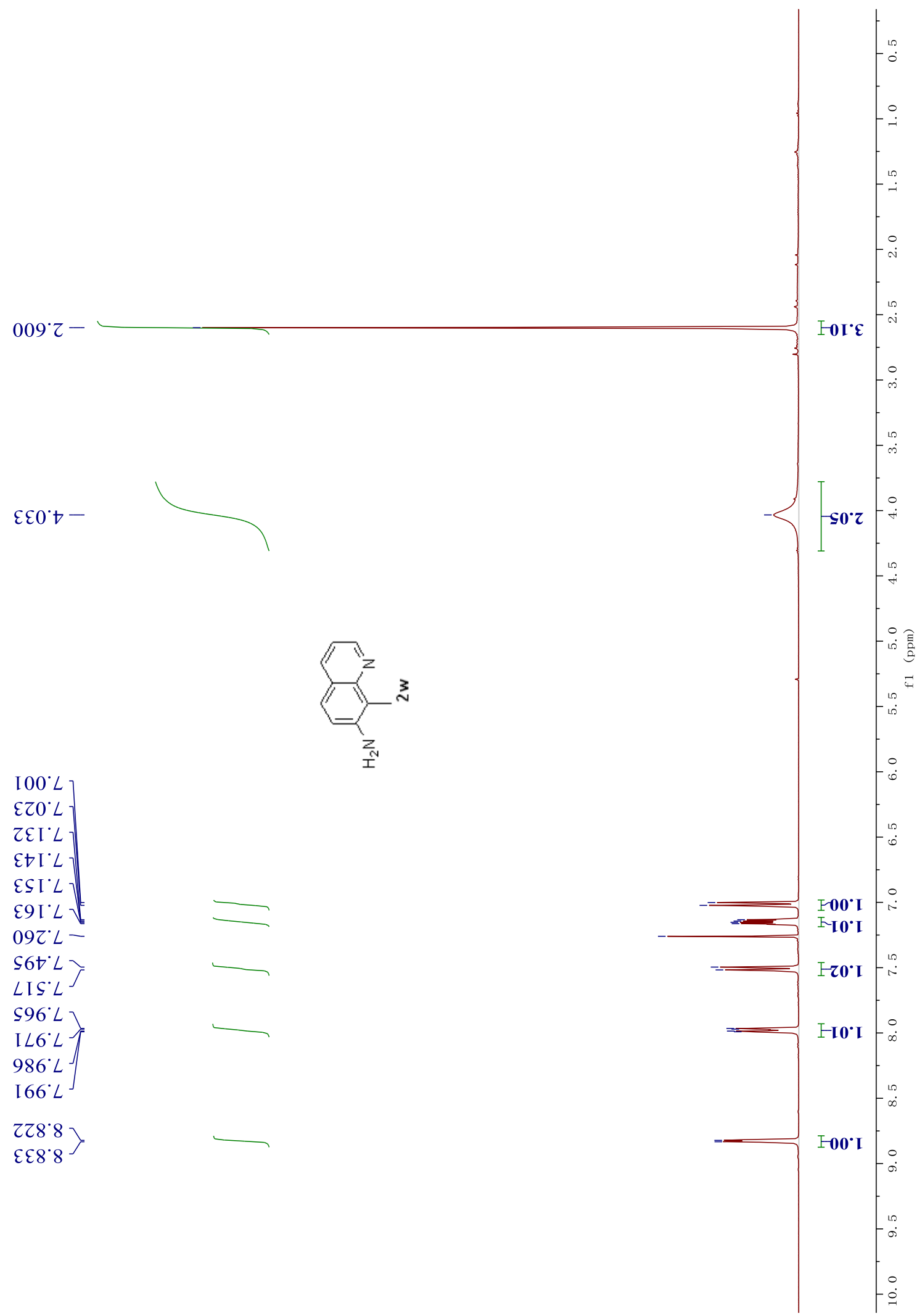


Z0E.0 I -

$278^{\circ} 9 L$

$09\left[\circ L \frac{1}{5}\right.$

$8 \angle t^{\circ} L L$

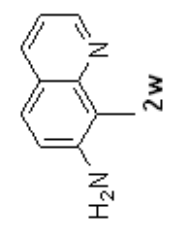

$\angle 9 L^{\circ} \nabla$ II てちでட I I 6t大 8 [ I 90s'zZI ELI'9ZI $J$

$L \varepsilon I^{\circ} 9 \varepsilon \mathrm{I}-$ t00 st ZLI'8t $ح$ ZZS.6tI

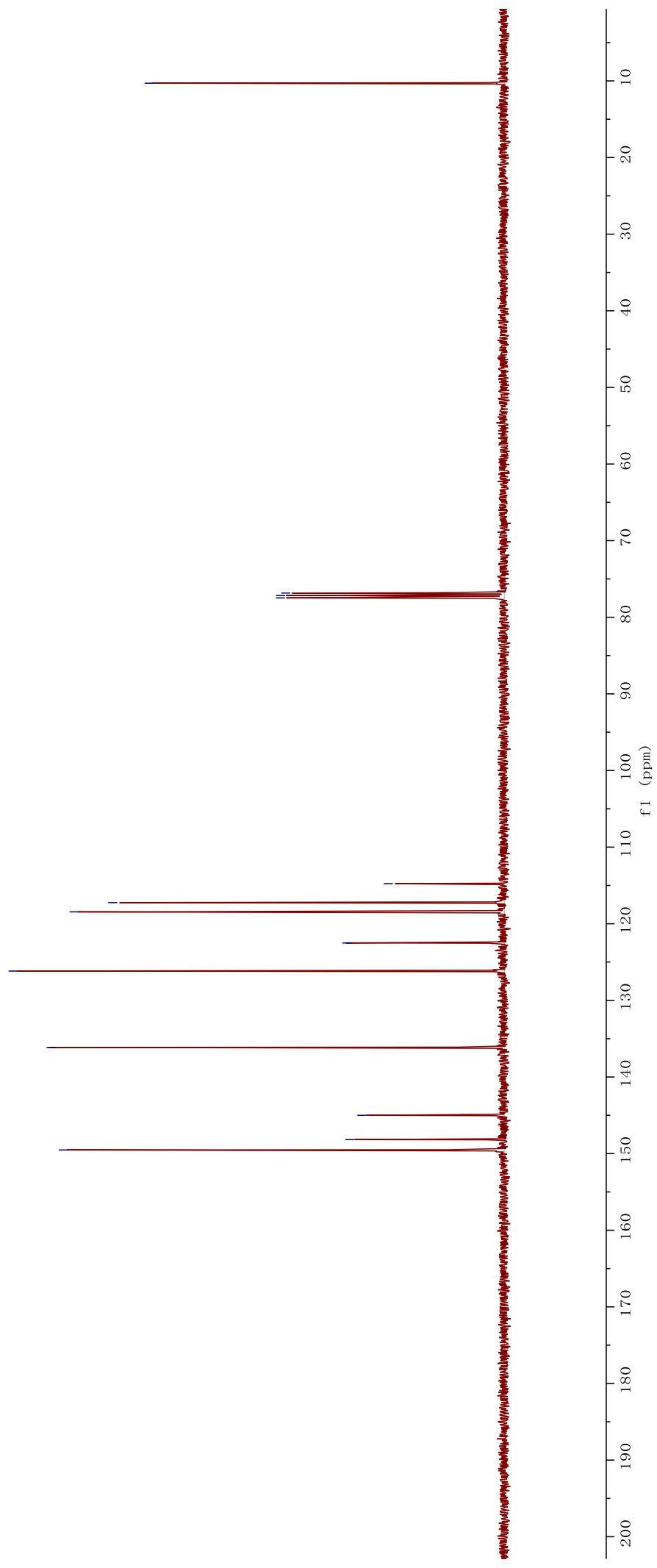


$8 L S^{\prime} 乙 \succ$

I $8 \varsigma^{\circ} Z$

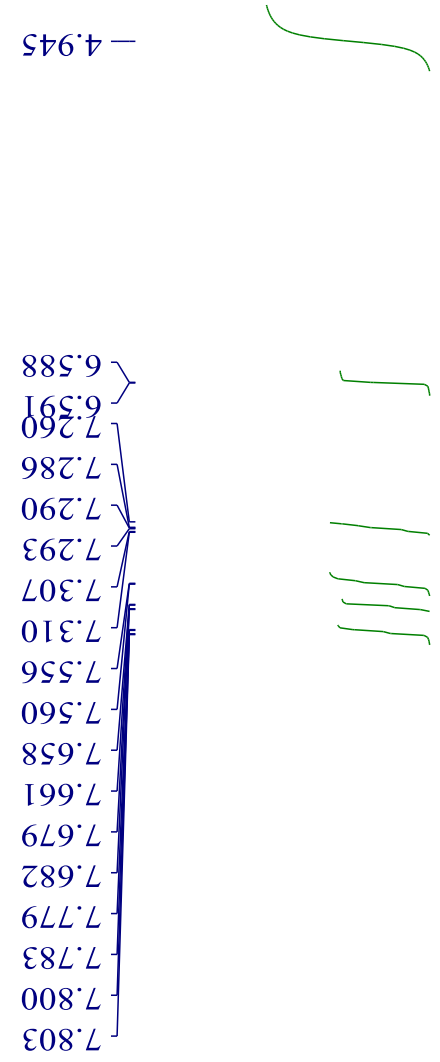

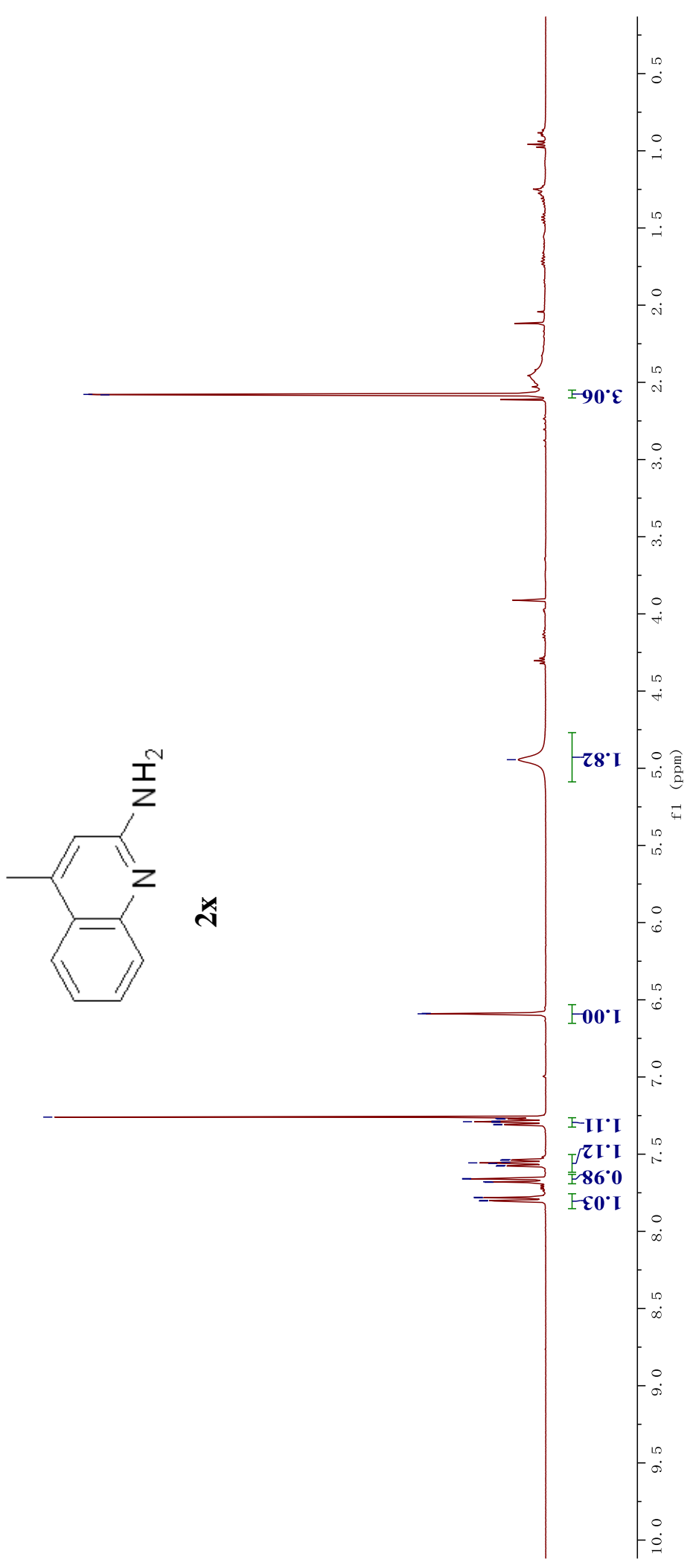


$66 L^{\circ} 8 \mathrm{I}-$
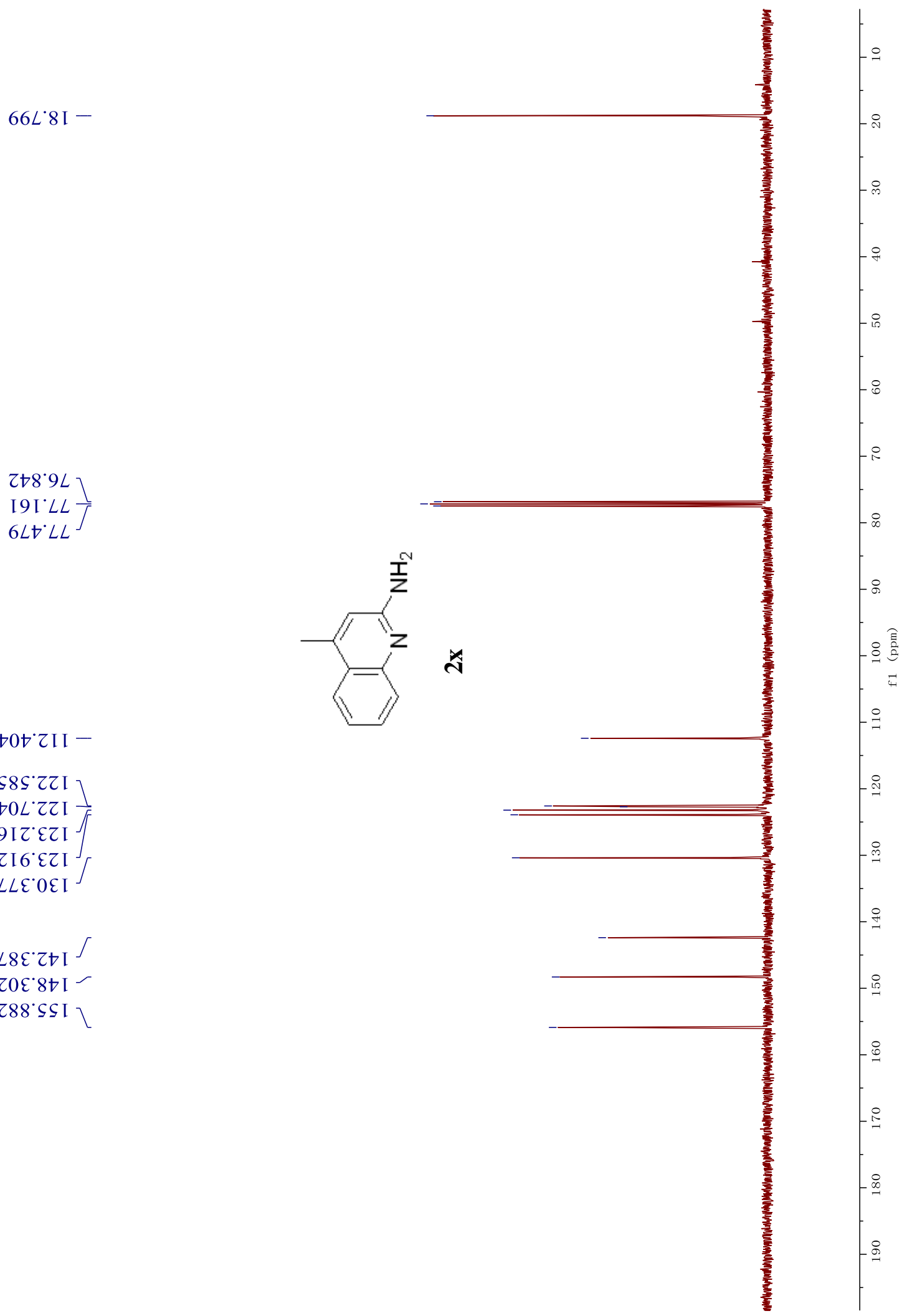


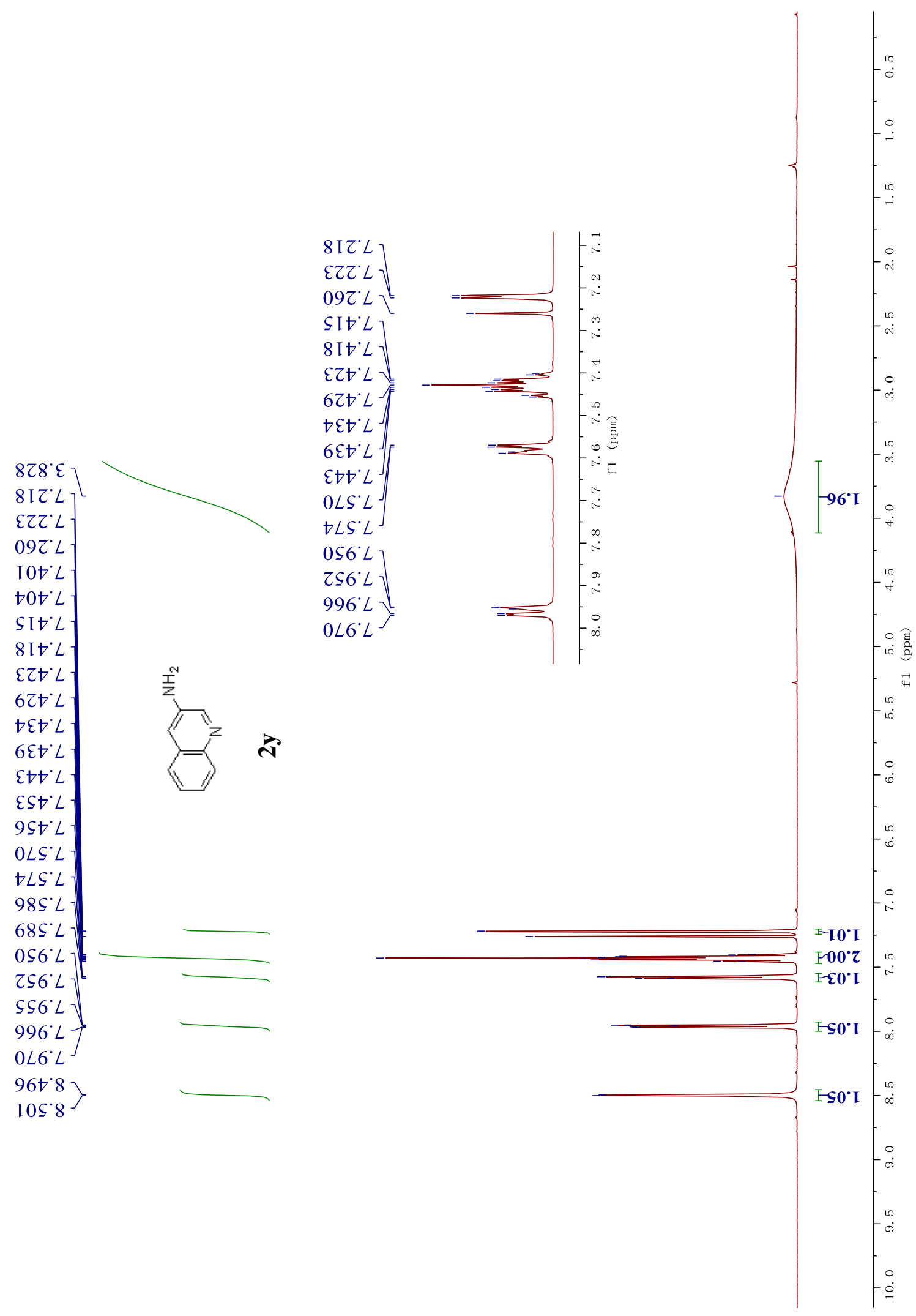


ยเวดว て๖8.9L

ยเวดว $09 \mathrm{I}^{\circ} L L \frac{\mathrm{T}}{\mathrm{T}}$

ยเगด $8 \angle t^{\circ} L L$

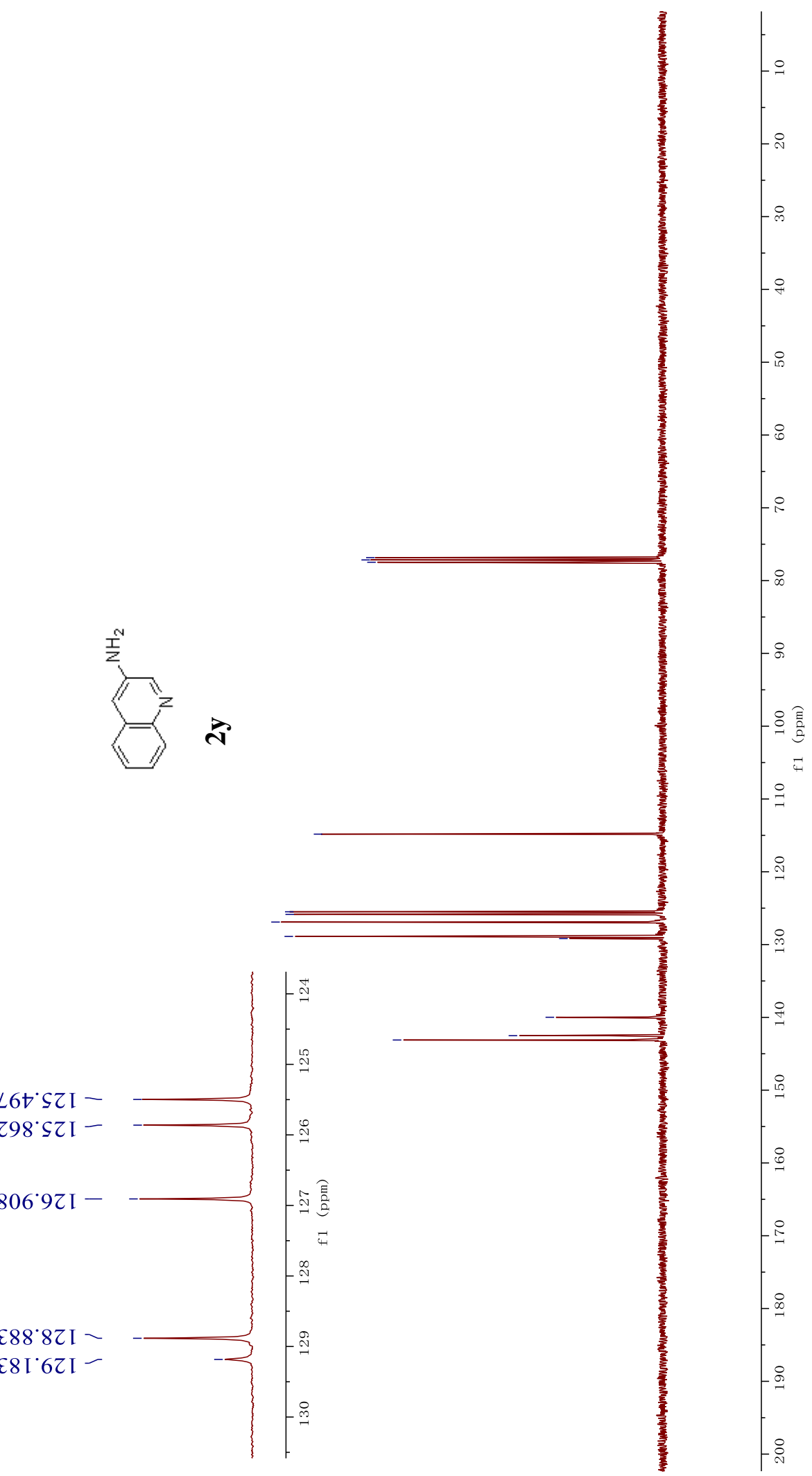

$\angle Z 8^{\circ} \circ \mathrm{II}$

$\angle 6 t^{\circ} \subseteq Z \mathrm{I}$

Z98 $\subseteq$ Z I

$806^{\circ} 9$ Z I

$\varepsilon 88^{\circ} 8 Z \mathrm{I}$

E8 I'6ZI

$986^{\circ} 6 \varepsilon \mathrm{I}$

LIS'て†I

I I I $\bullet \triangleright$ I

Z98. ऽZI - 


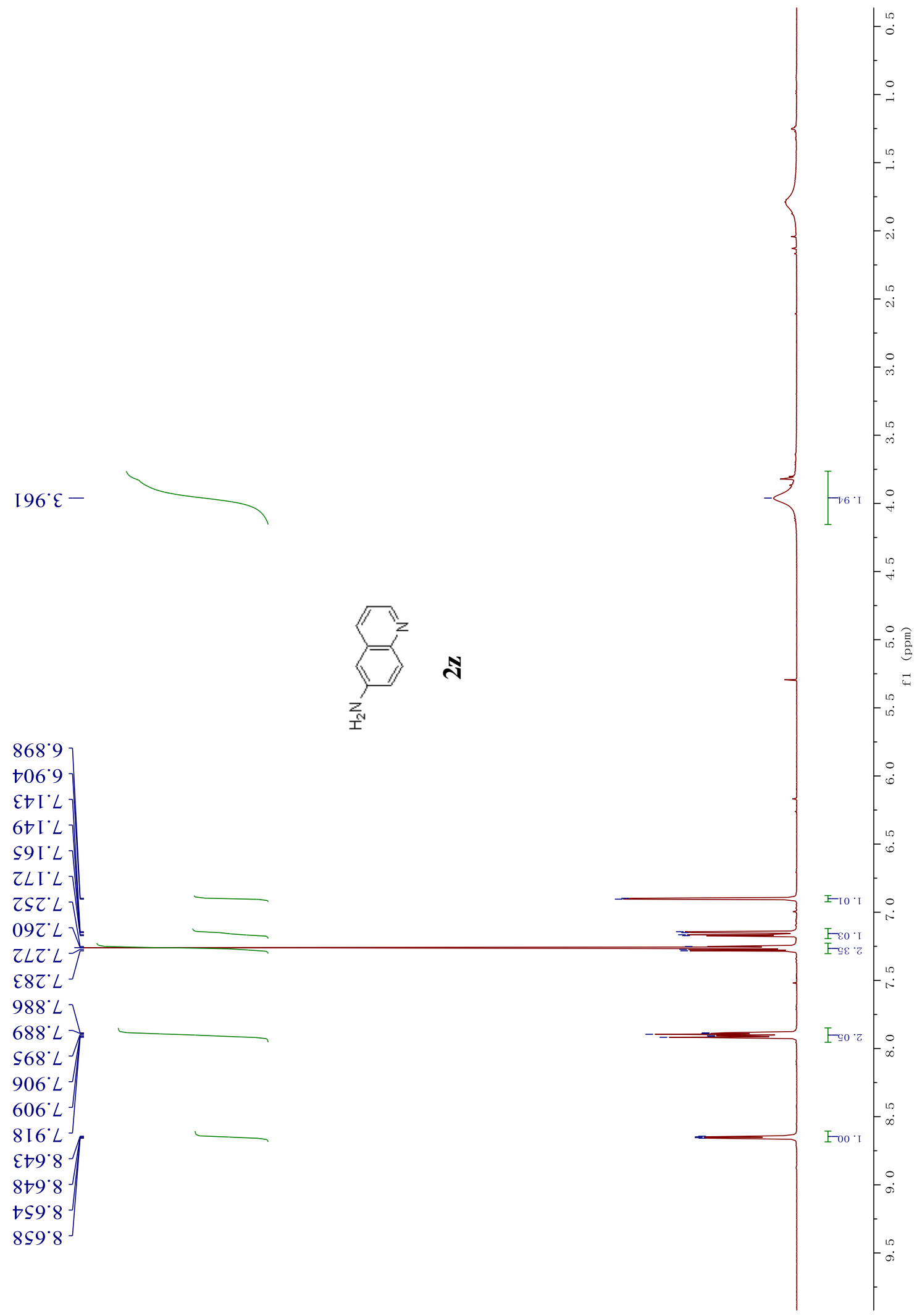



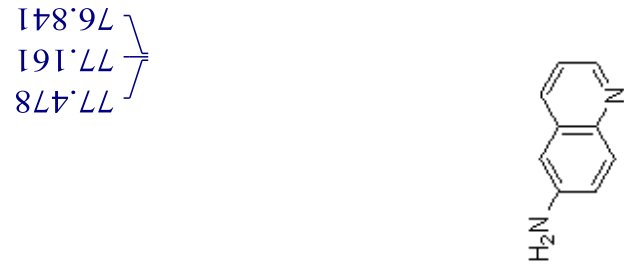

ก

$86 I^{\circ} \angle 0 \mathrm{I}-$

$\left.\begin{array}{l}\angle 8 Z^{\circ} \mathrm{IZI} \\ \succ 09^{\circ} \mathrm{IZI}\end{array}\right\}$

It $L^{\circ} 6 Z \mathrm{I}$

S6 [ ${ }^{\circ} 0 \varepsilon \mathrm{I}^{\nearrow}$

$\angle \forall L^{\circ} \varepsilon \varepsilon I^{\prime}$

$8 \varepsilon I^{\circ} \varepsilon \nabla \mathrm{I} \backslash$

8 t8*t十I -

$86 t^{\circ} 9 t \mathrm{I}$

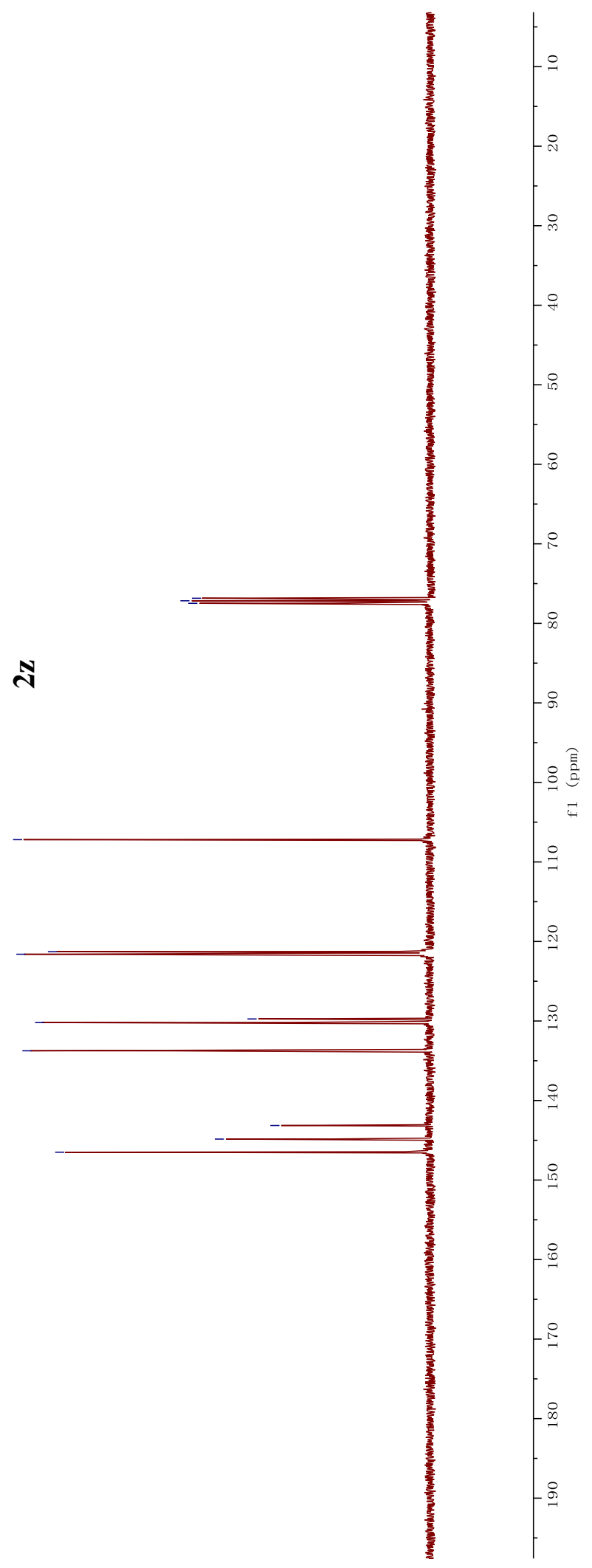


$899^{\circ}$ Z

$2 \succ 0 \circ$

$68^{\circ} 9$

$\varepsilon 06^{\circ} 9$

816.9

$\downarrow 26^{\circ} 9$

$\rightarrow I 00^{\circ}-$

SEO $0^{\circ}$

$8 \rightarrow\left[{ }^{\circ} L\right.$

$\left.\varepsilon S I^{\circ} L\right]$

$09 \tau^{\circ} L$

$S+S^{\circ} L$

$\angle 9 S^{\circ} L$

$9 S 8^{\circ} \mathrm{L}$

$\angle L 8^{\circ} \mathrm{L}$

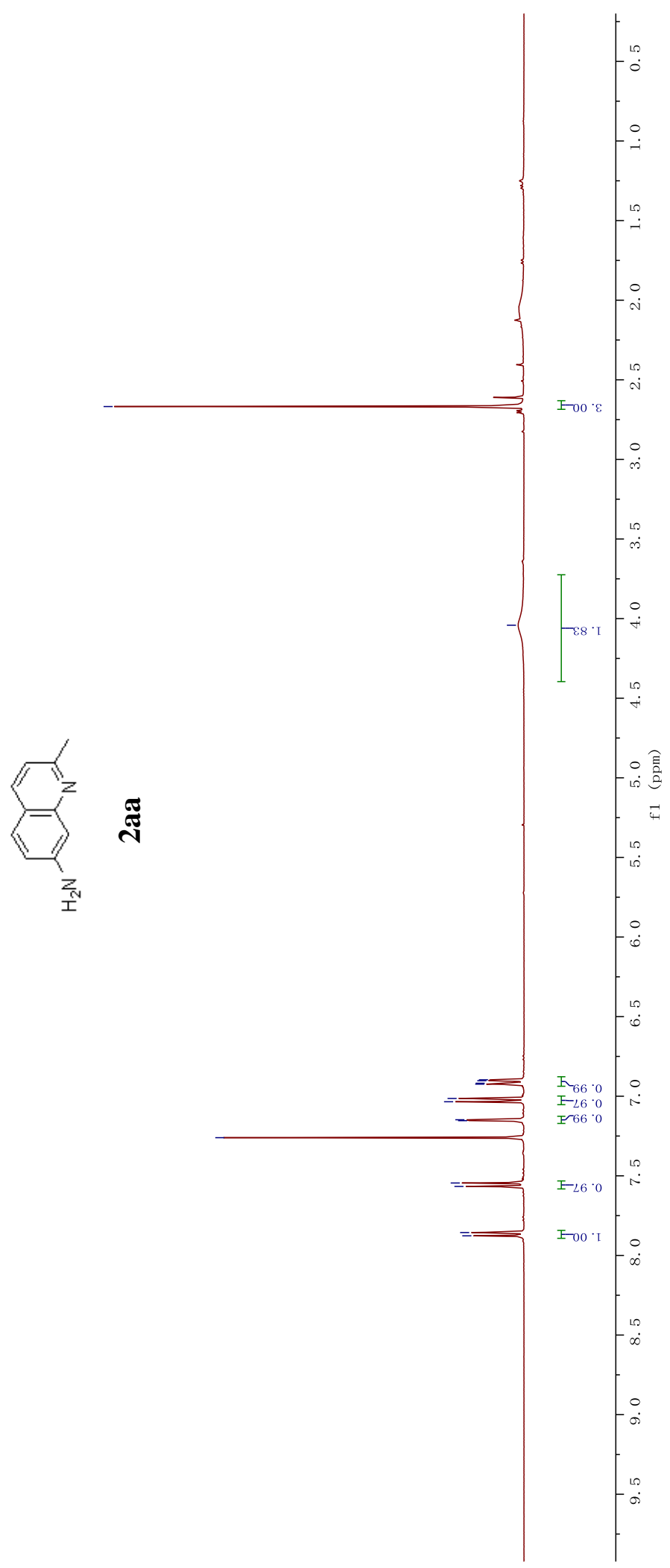


$\lfloor+0 \cdot \varsigma r-$

Z†8.9L

$09 I^{\circ} \angle L I$
$8 \angle \nabla^{\circ} L L$

It $\mathcal{8} 80$ I -

EZ9. LII

$\mathrm{I} t \varepsilon^{\circ} 8 \mathrm{II}$

LIZ'0ZI

$\angle Z S^{\circ} 8 Z I-$

$806^{\circ}$ SEI -

$\varepsilon \mathrm{I0} 80^{\circ} \mathrm{I}$

$z † \varepsilon^{\circ} 6 \triangleright I^{\prime}$

$6 \dagger 6.8 S \mathrm{I}-$

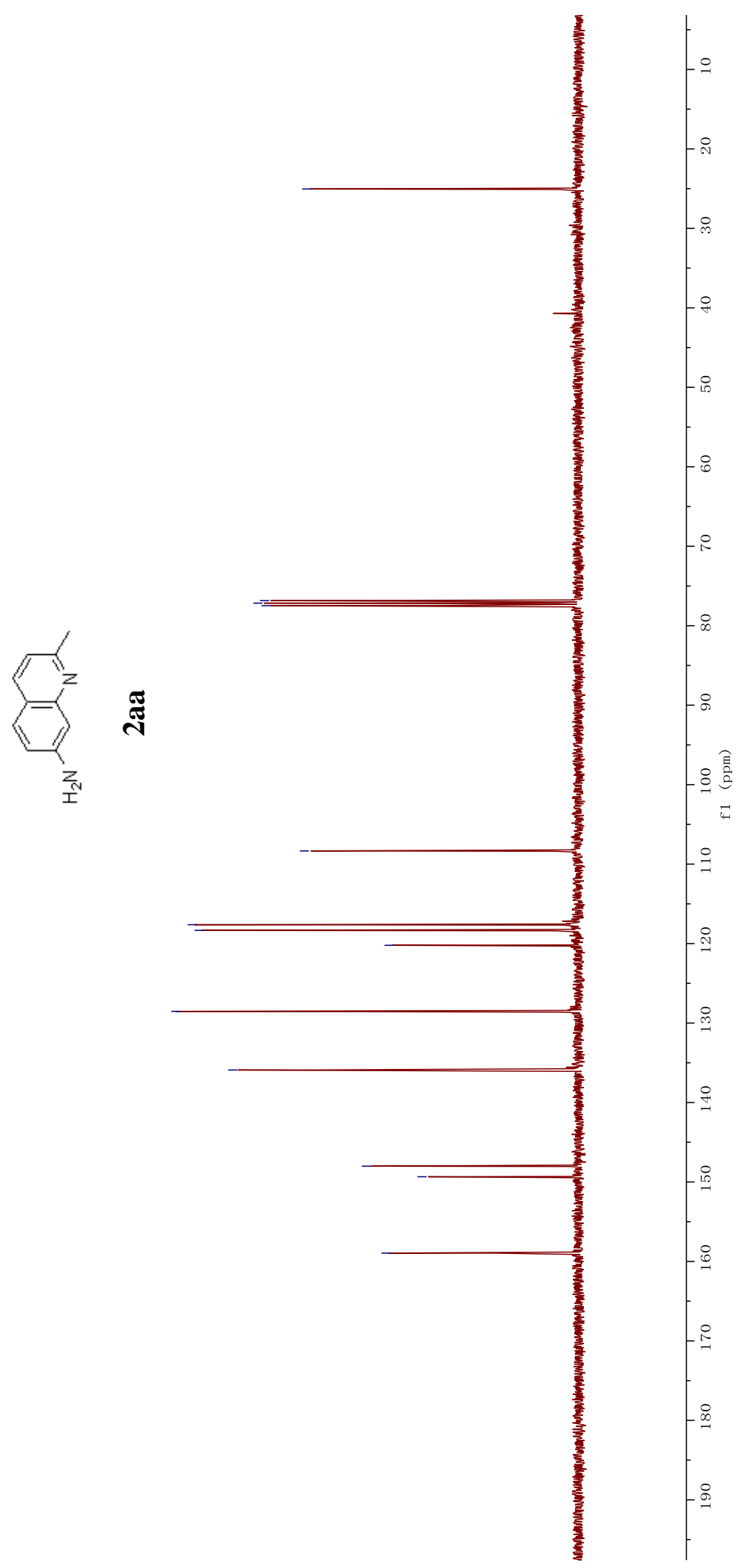




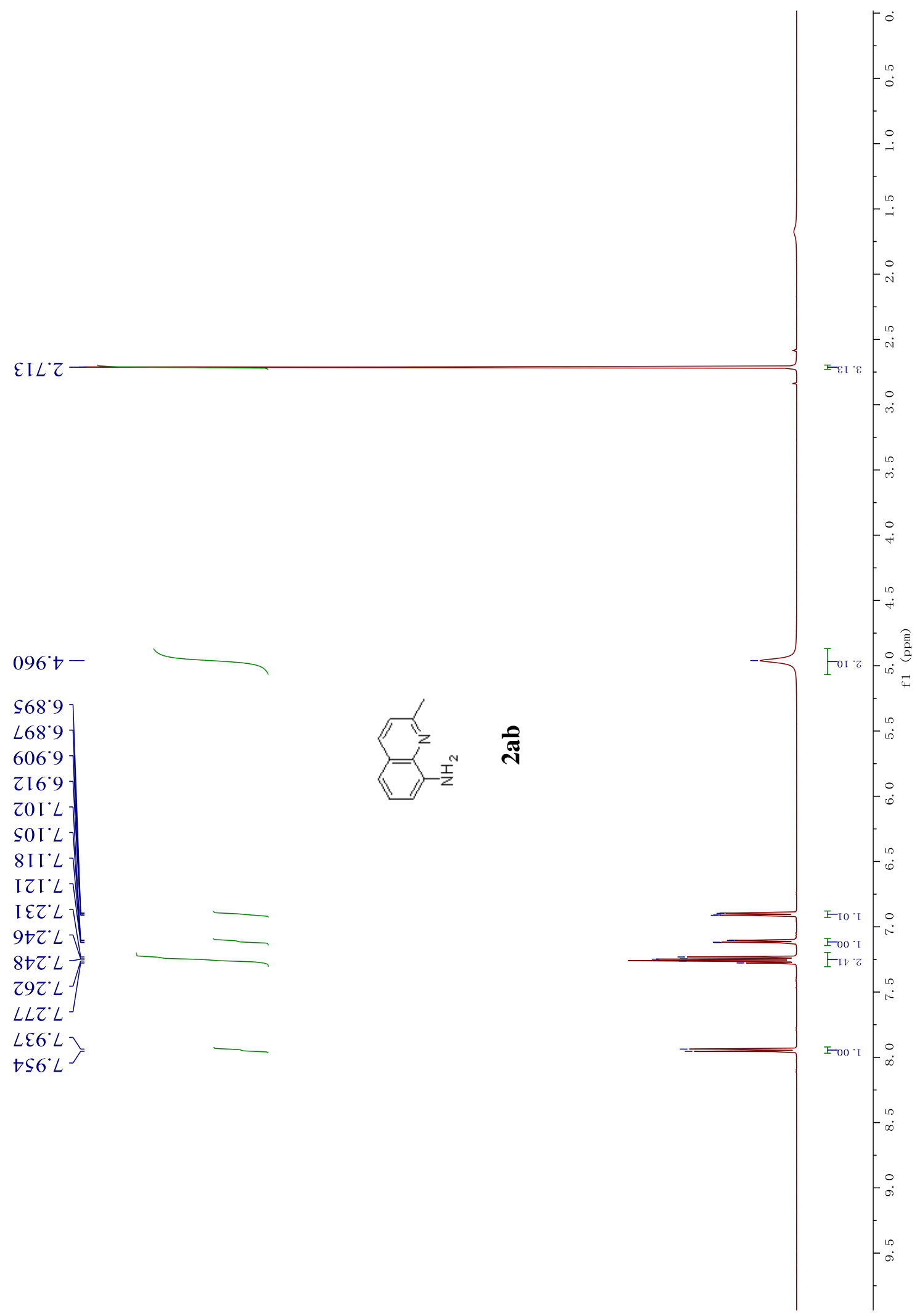


6IZ'ৎZ-

$\varepsilon 06^{\circ} 9 L$
$8 S I^{\circ} L L T$

ZItTLL

กิ

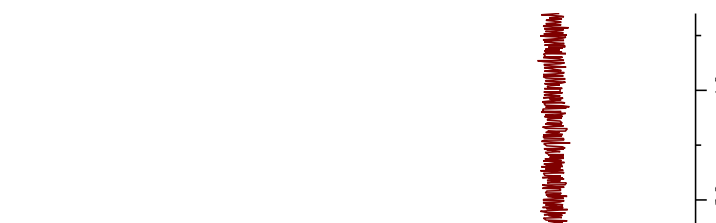

t0I.0 I I

Z†8.

60 I' $Z Z \mathrm{I}$

ऽоદ. $9 Z \mathrm{I}$

$888^{\circ} 9$ I I $^{\circ}$

$\pitchfork t 0^{\circ} 9 \varepsilon \mathrm{I}-$

St $8^{\circ} \angle \varepsilon \mathrm{I}$

6Et $\varepsilon \sqcup \mathrm{I}-$

L60.9SI -

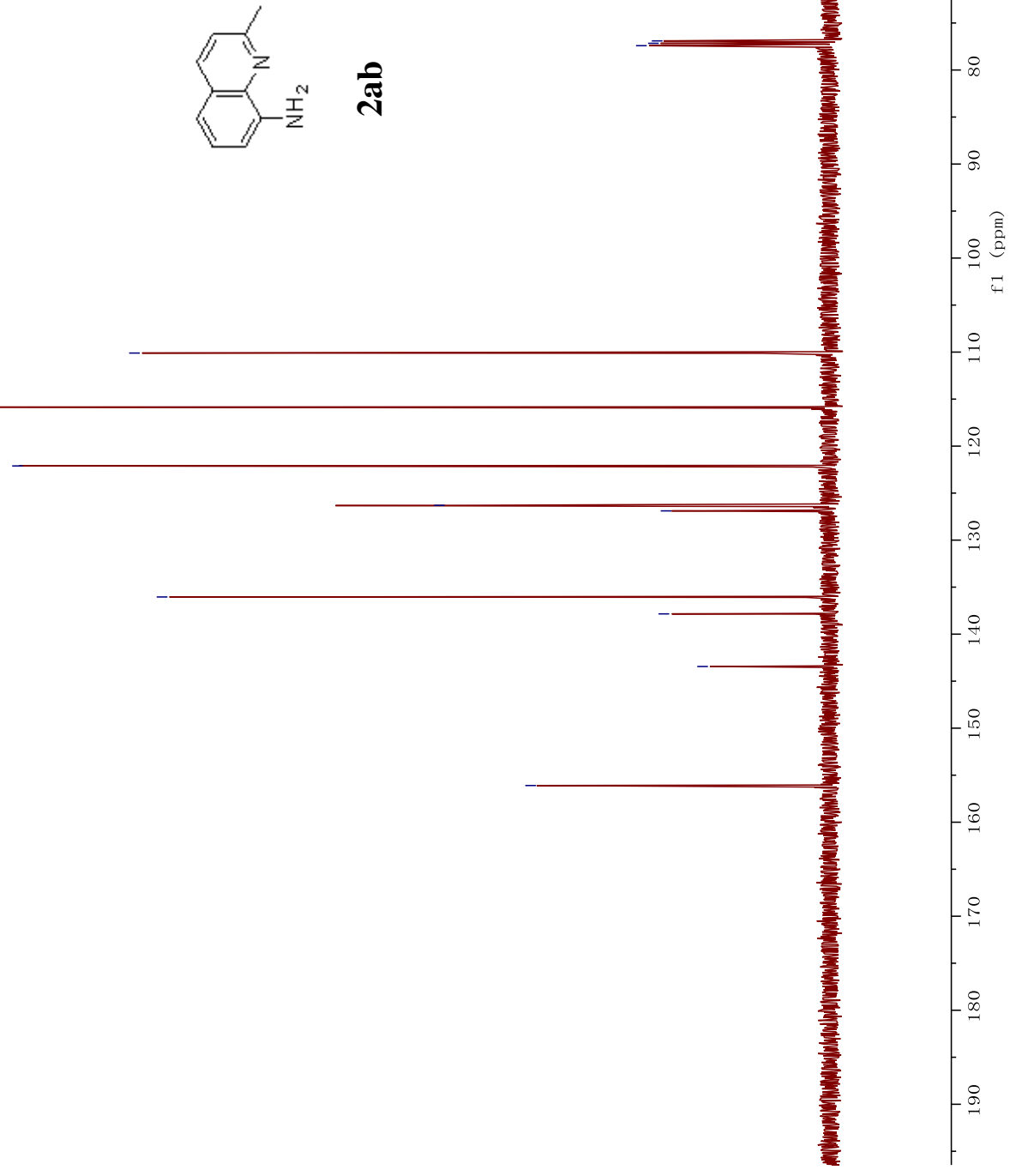



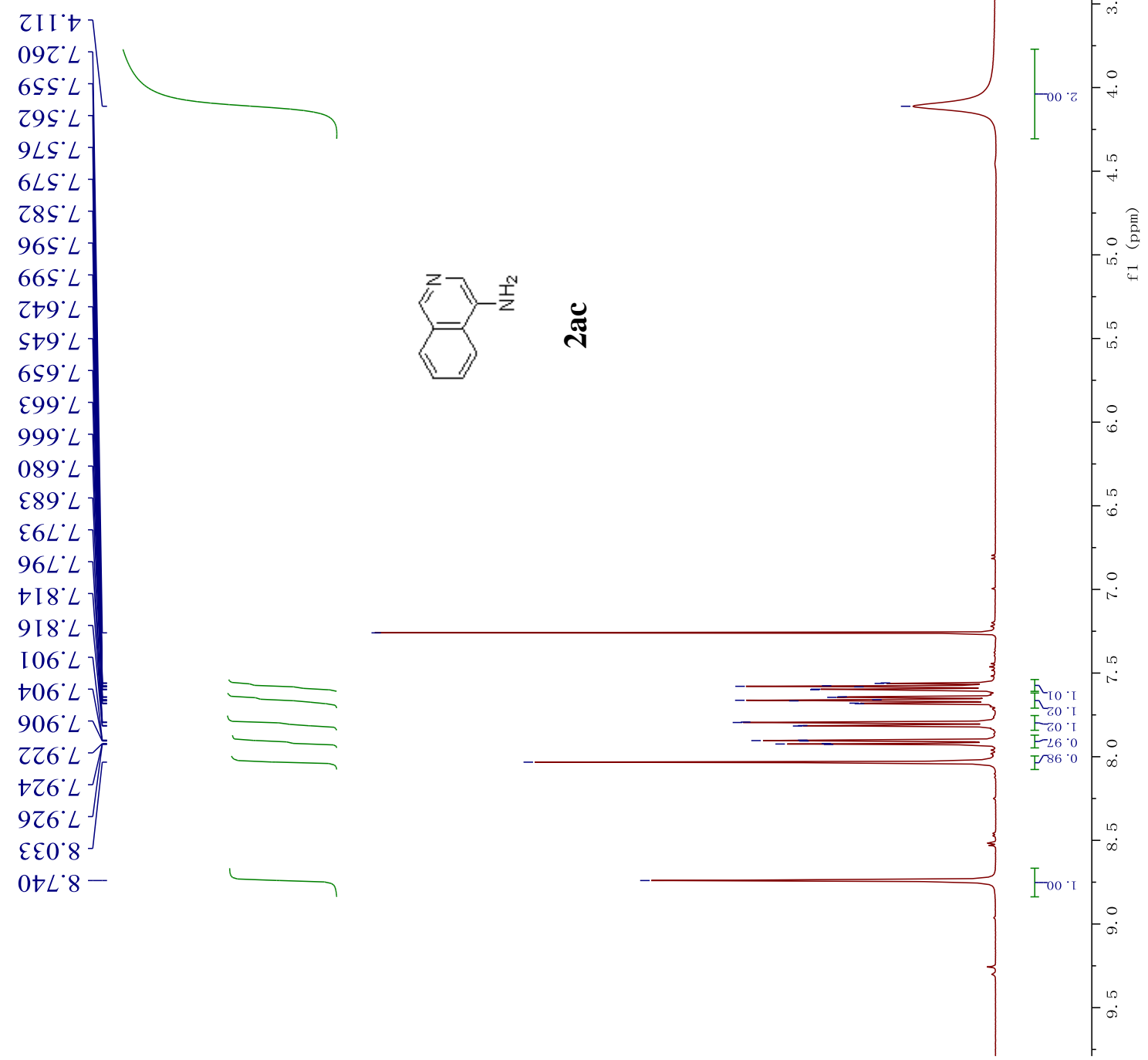
$\$ 06^{\circ} 9 \mathrm{~L}$

SI I S $^{\circ} L L T$

8 I I $^{\circ} 0$ Z I

$9 \triangleright 0.9 Z \mathrm{I}$

$\downarrow \varepsilon 0^{\circ} L Z[$

$\angle Z L^{\circ} \angle Z$ I

$886^{\circ} \mathrm{LZI}$

$629^{\circ} 8$ ZI

$8 S 6^{\circ} 8 Z \mathrm{I}$

LII $L E$ I -

996て๖Iー

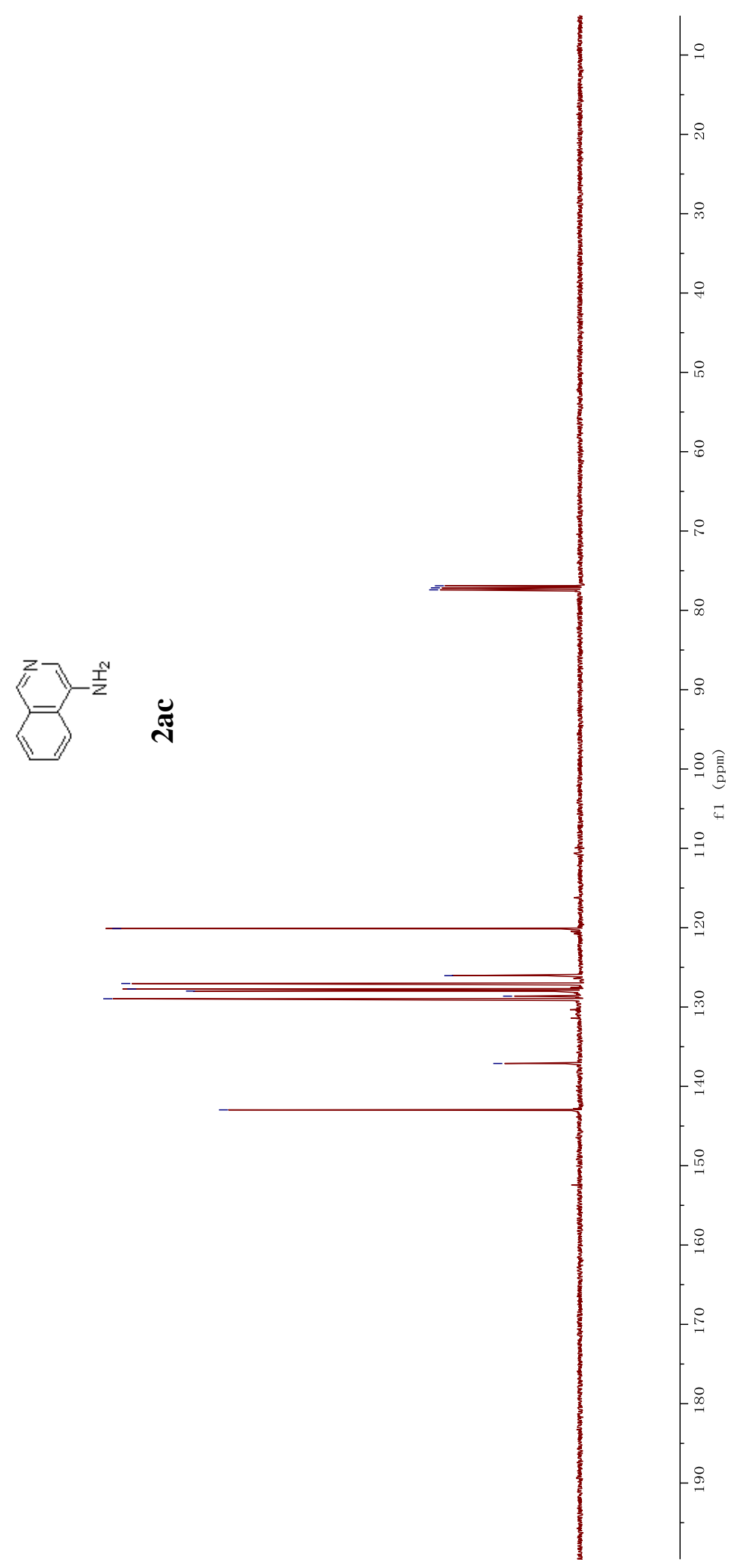




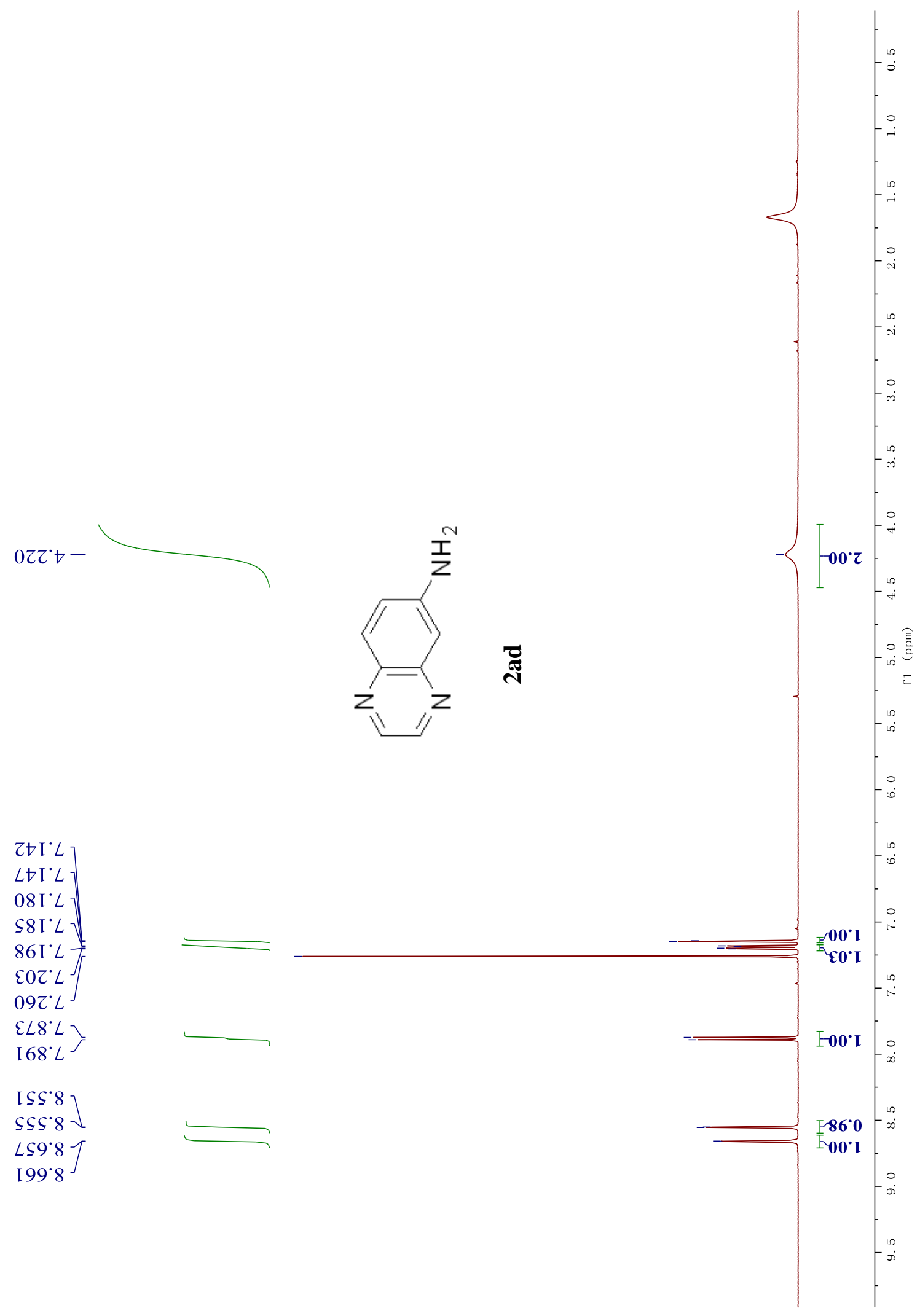


$\left.\begin{array}{l}\text { Z๐ } 8^{\circ} 9 L \\ 09 I^{\circ} L L \frac{J}{8 L ナ^{\circ} L L}\end{array}\right]$

0E6 $6^{\circ} \angle 0$ I -

oย乙`てZI -

SLプ0EI -

$980^{\circ} 8 \varepsilon \mathrm{E}$

$0 Z 0^{\circ} \mathrm{I} t \mathrm{I}$

$996^{\circ} \nabla t$

$\nabla 60^{\circ} \varsigma$ I

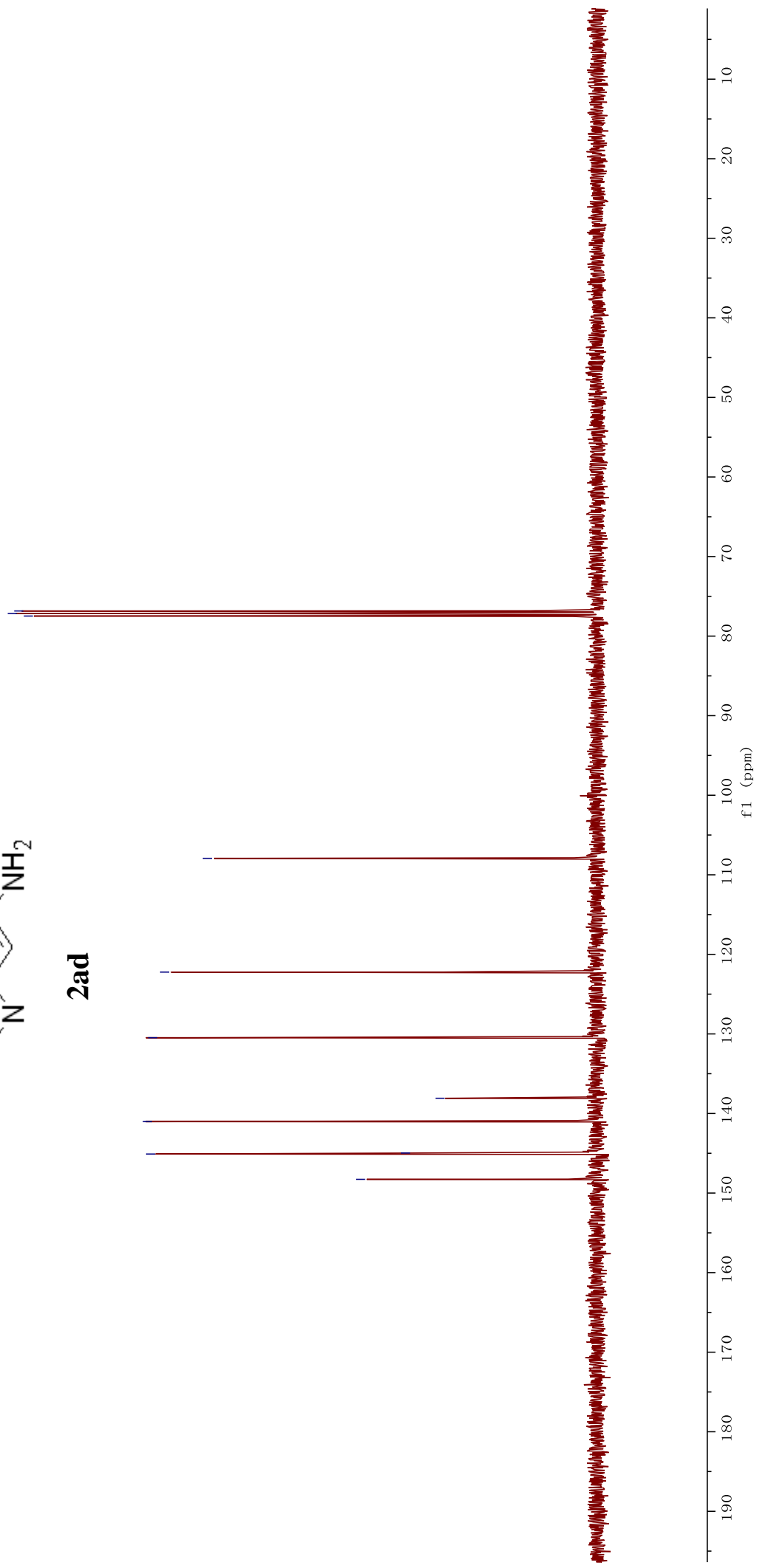




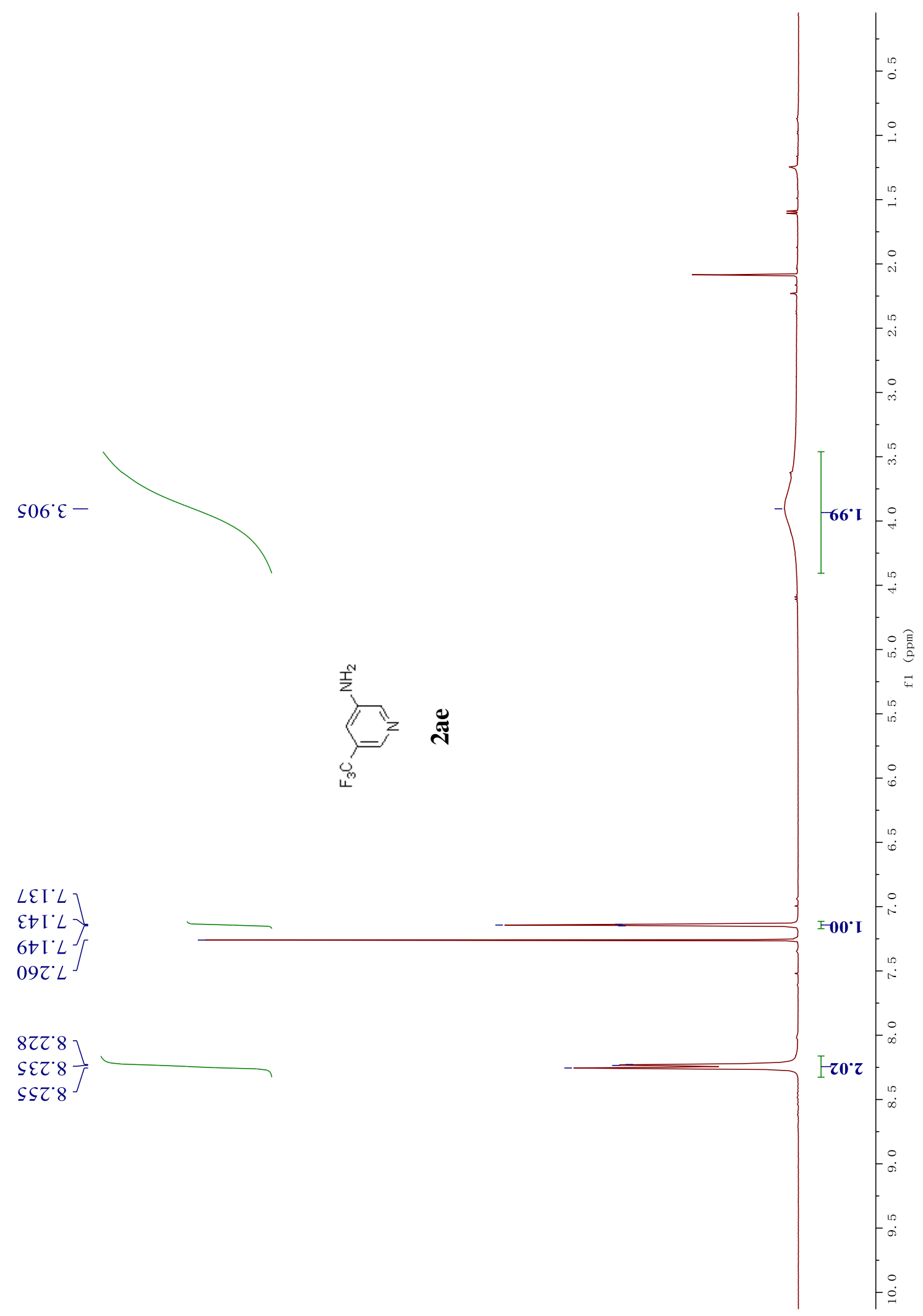




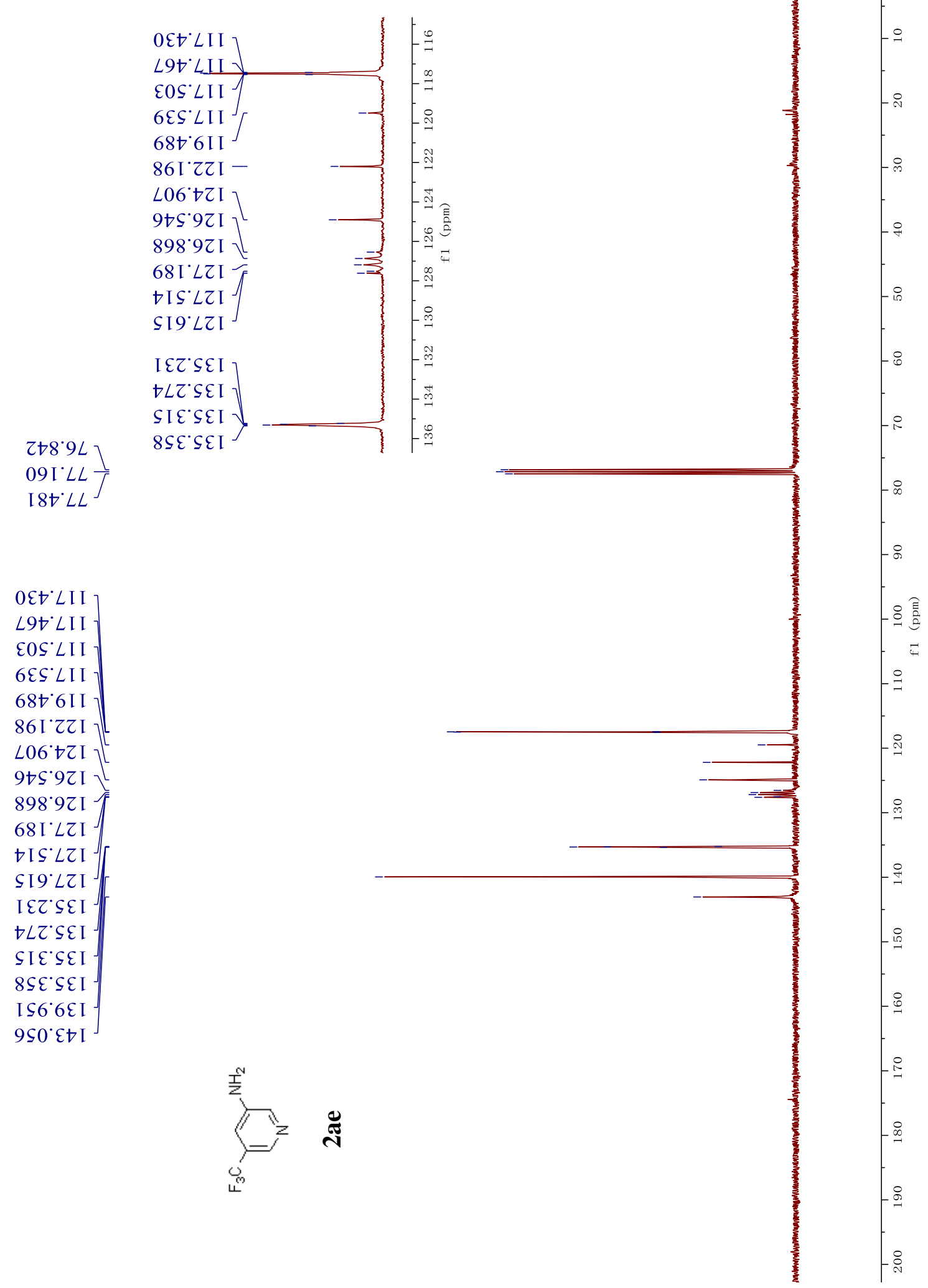




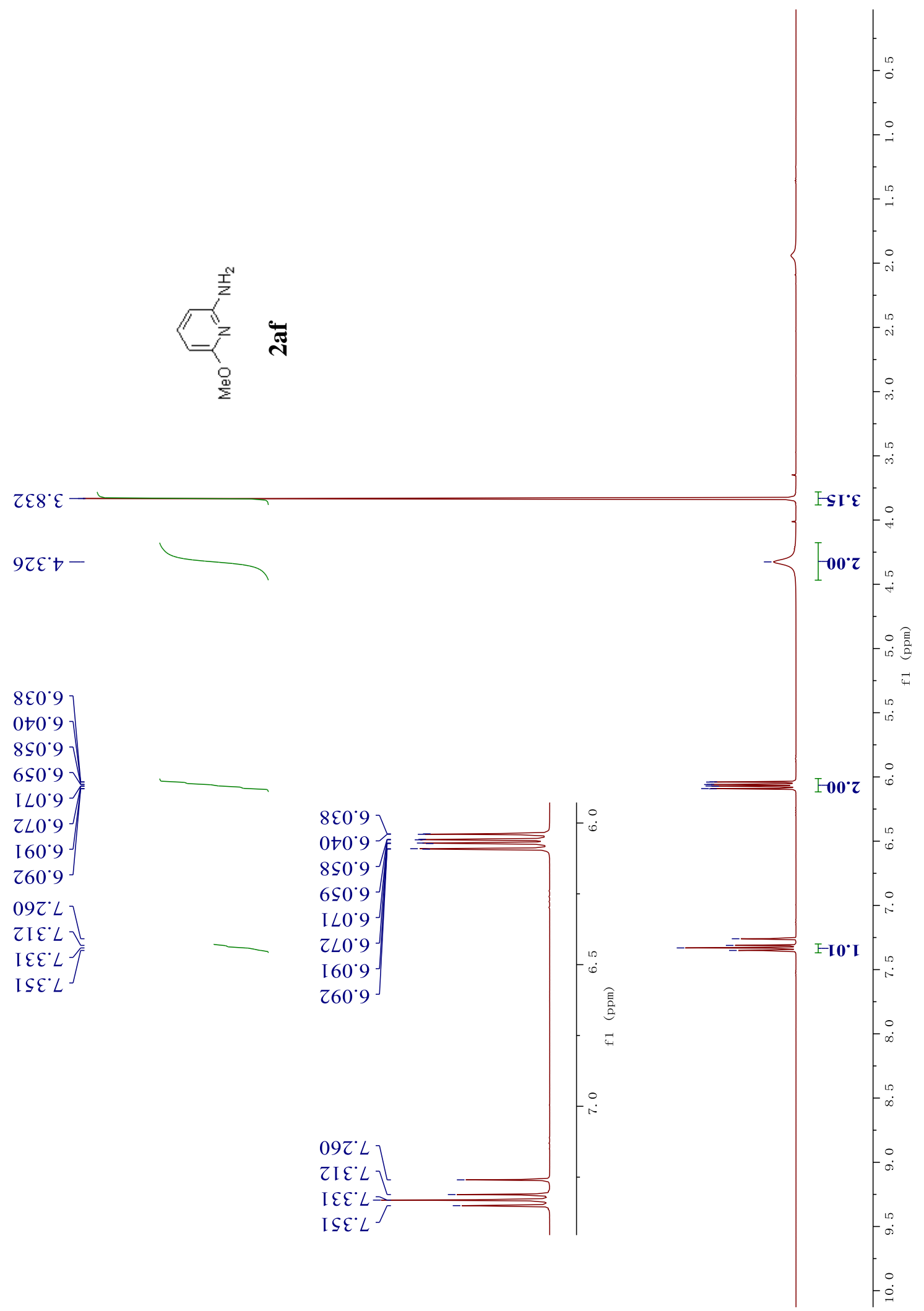




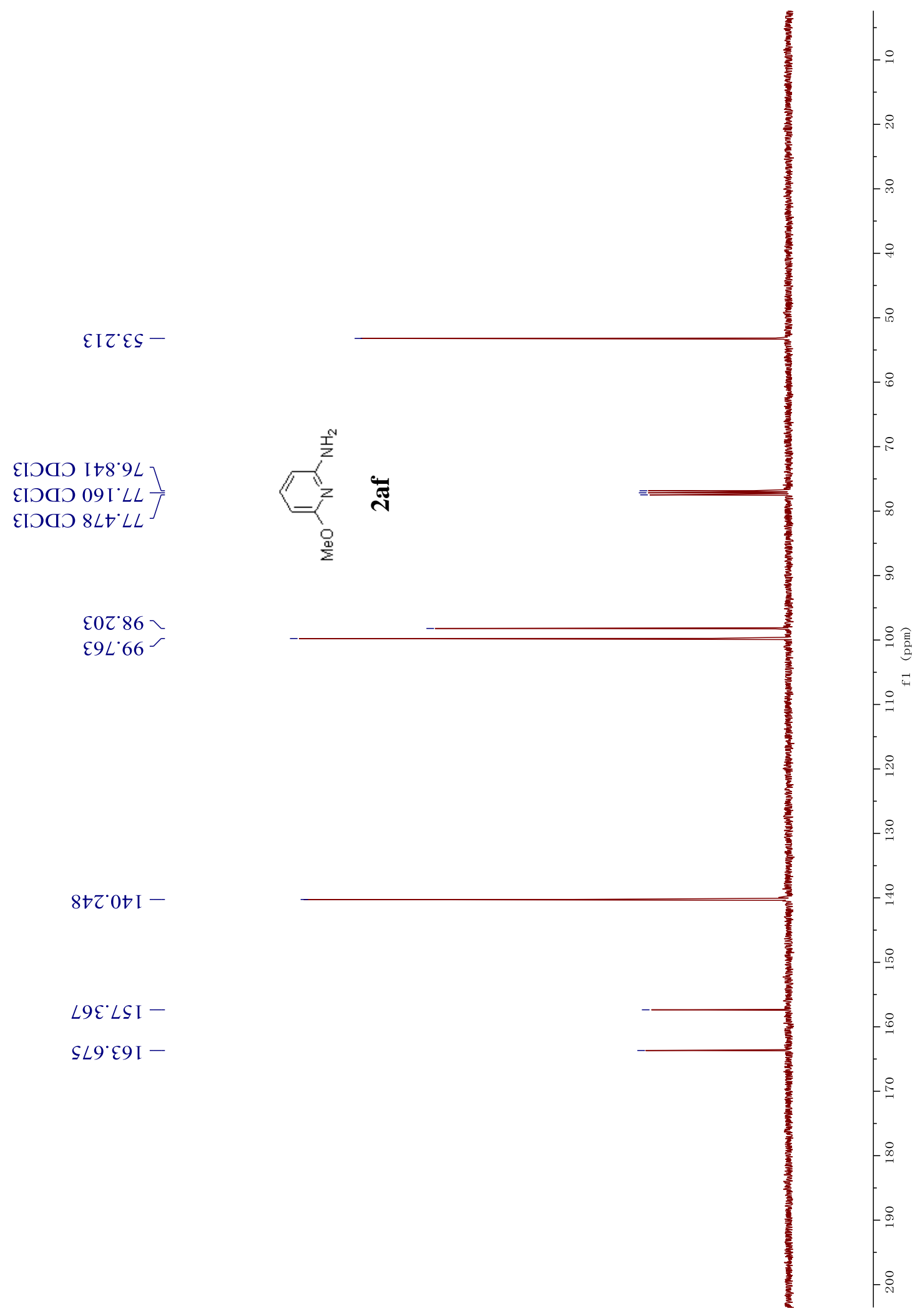




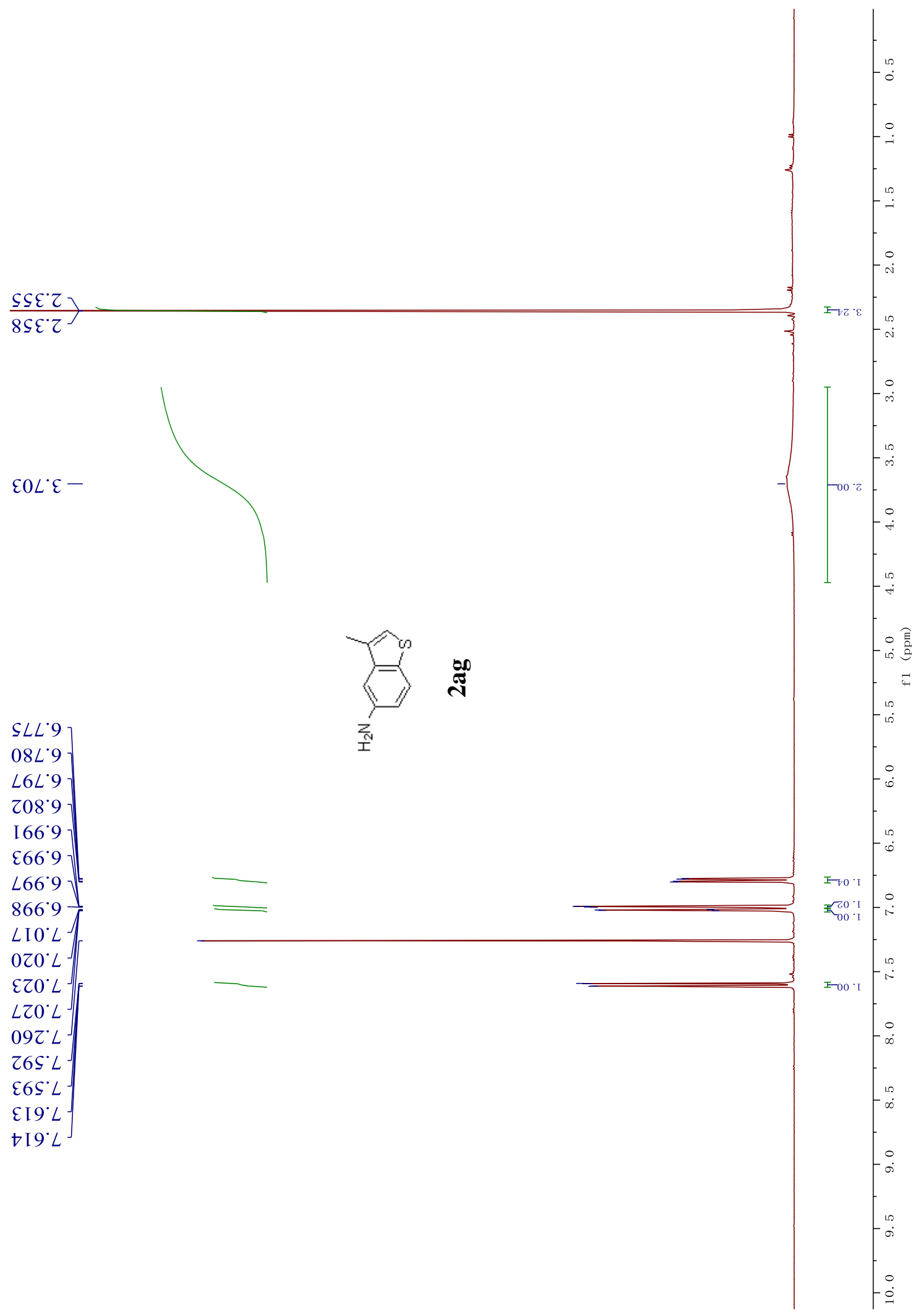


L6L'EI -

[ $78^{\circ} 9 L$

I9 I $^{\circ} L L T$

$6 L D L L$

† $9^{\circ} 90 \mathrm{I}-$

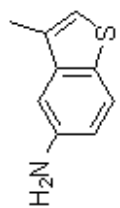

กู

Z†S $\downarrow$ II -

98 ['ZZI]

†0I. $\varepsilon Z \mathrm{I}-$

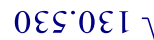

S9I.IEI -

S8L'0tI -

$\varsigma \varsigma \mathcal{E}^{\circ} \mathcal{E}$ I -

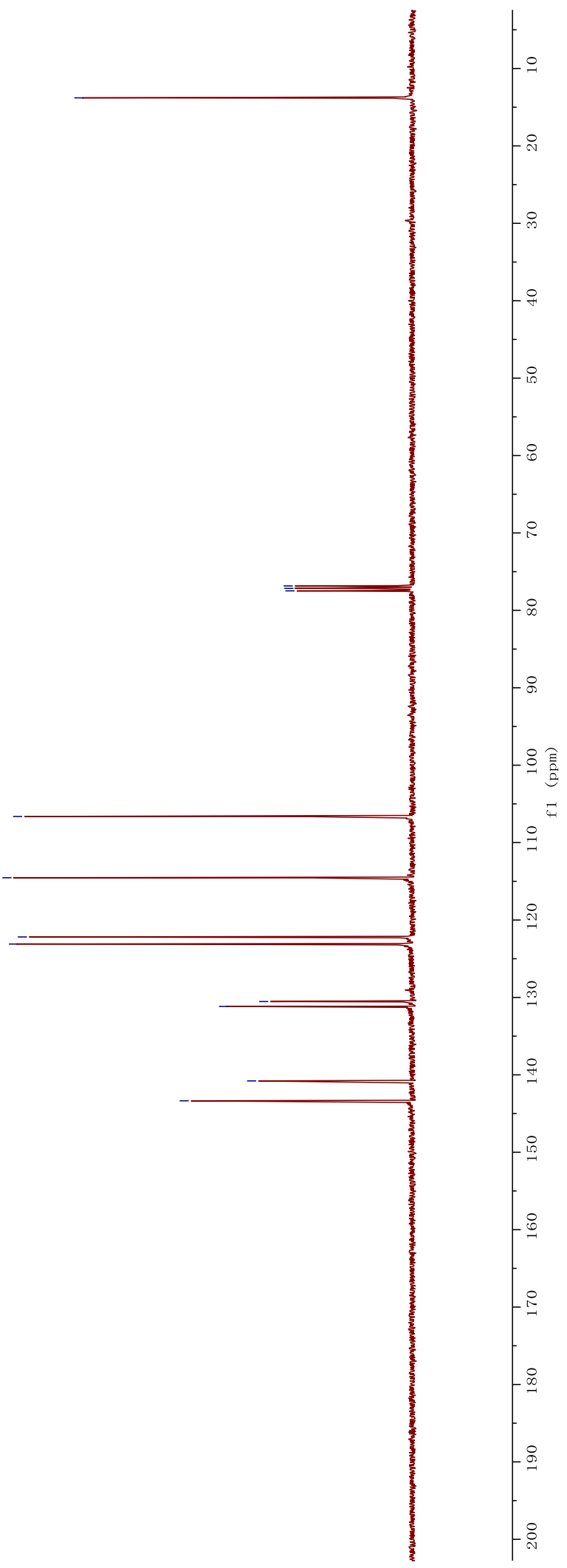




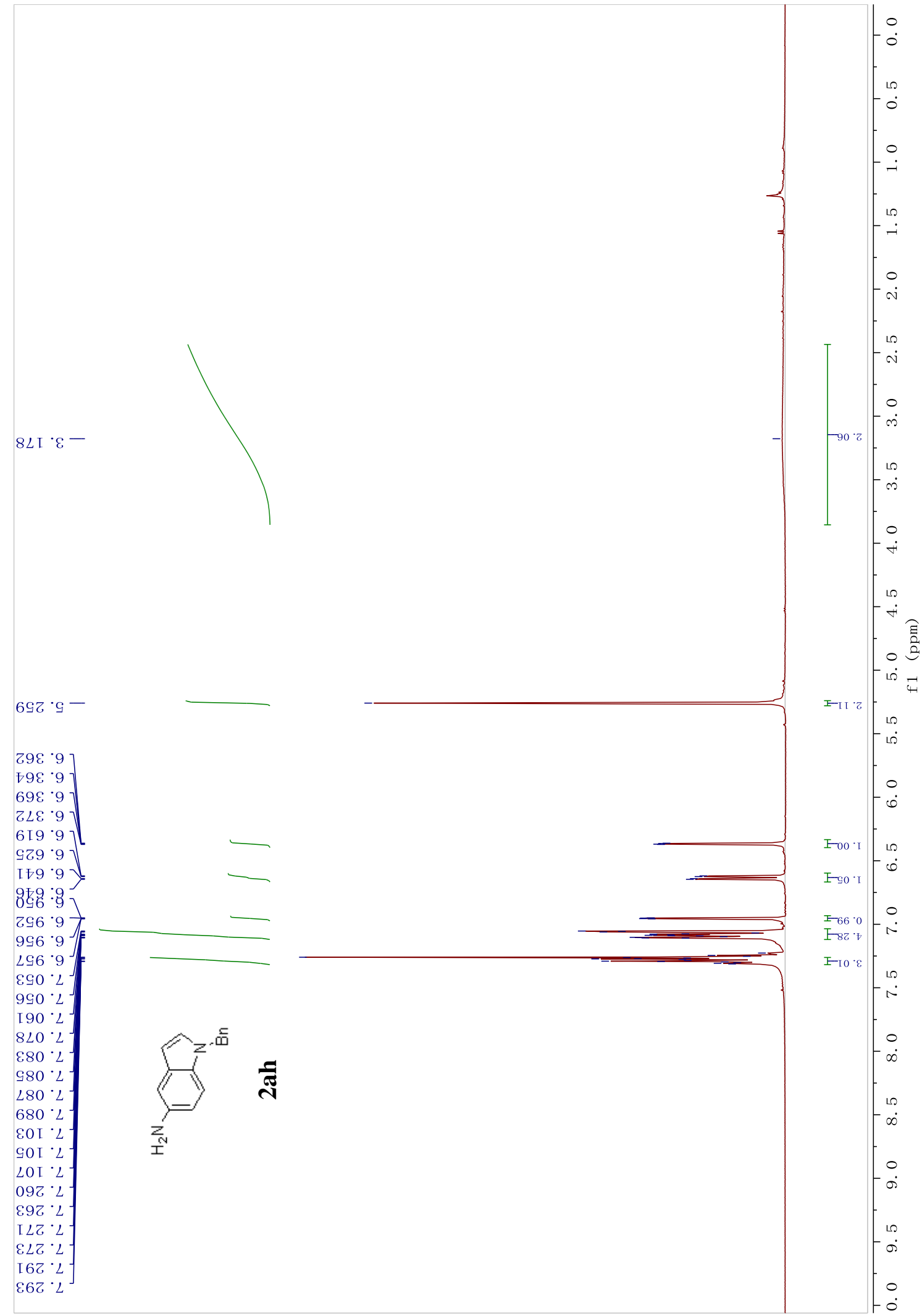


$60 \varepsilon \cdot 09-$

$906^{\circ} 9 L$
$091 \cdot L L T$

IID $L L$

067.00I

$796^{\circ} \mathrm{GOI} ح$

9[6 OLI]

9LL'ZII

$8 Z 8^{\circ} 9 Z$ [ ]

0 [9 $2 Z$ I]

$838 \cdot 8 Z \mathrm{~L}-$

6DL 6ZI

$\nabla Z 9 \cdot \mathrm{I} \varepsilon \mathrm{I}$

$706^{\circ} \mathrm{LEI} \sim$

$98 \mathrm{6} \cdot 6 \varepsilon \mathrm{I}$

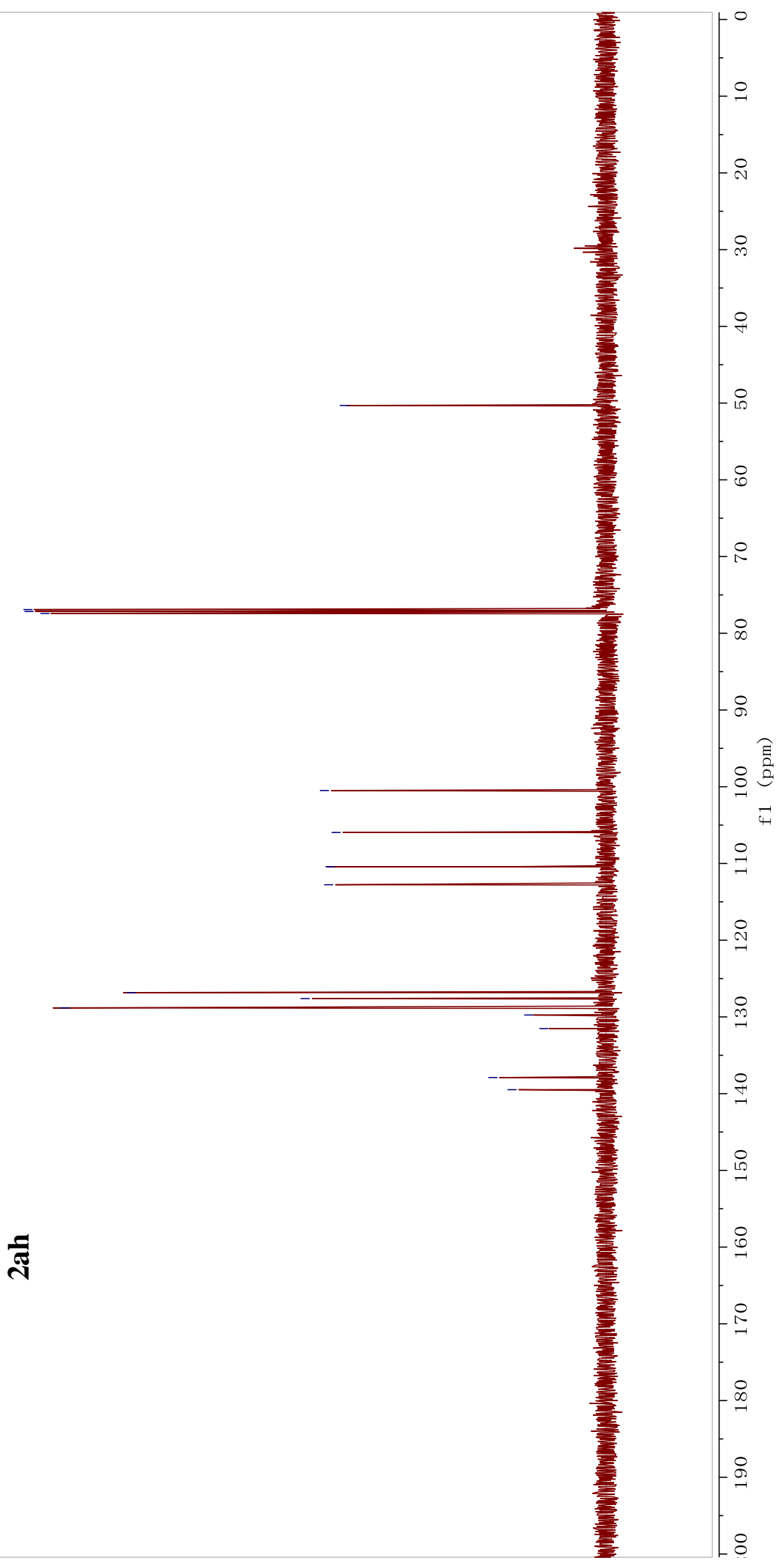




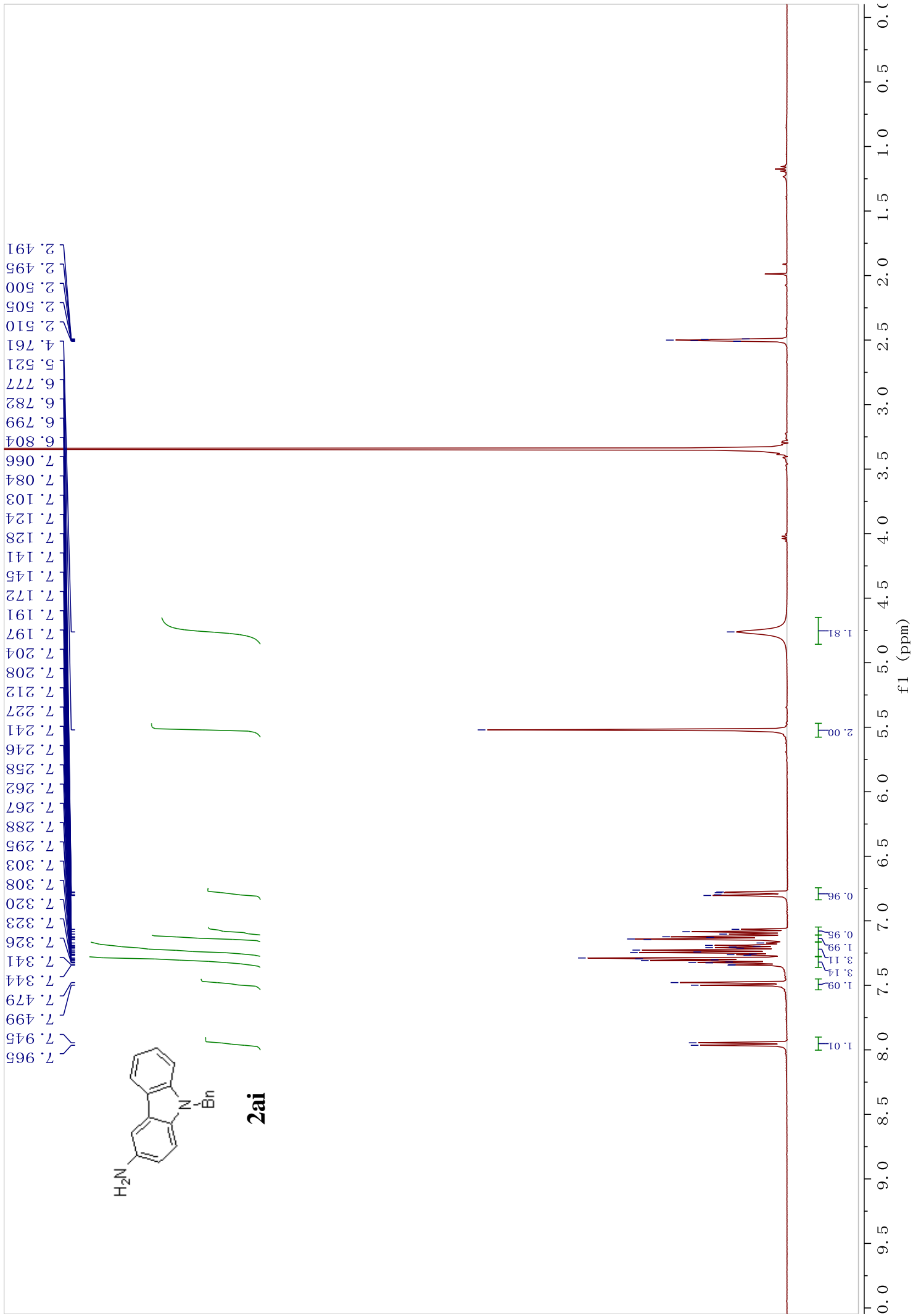




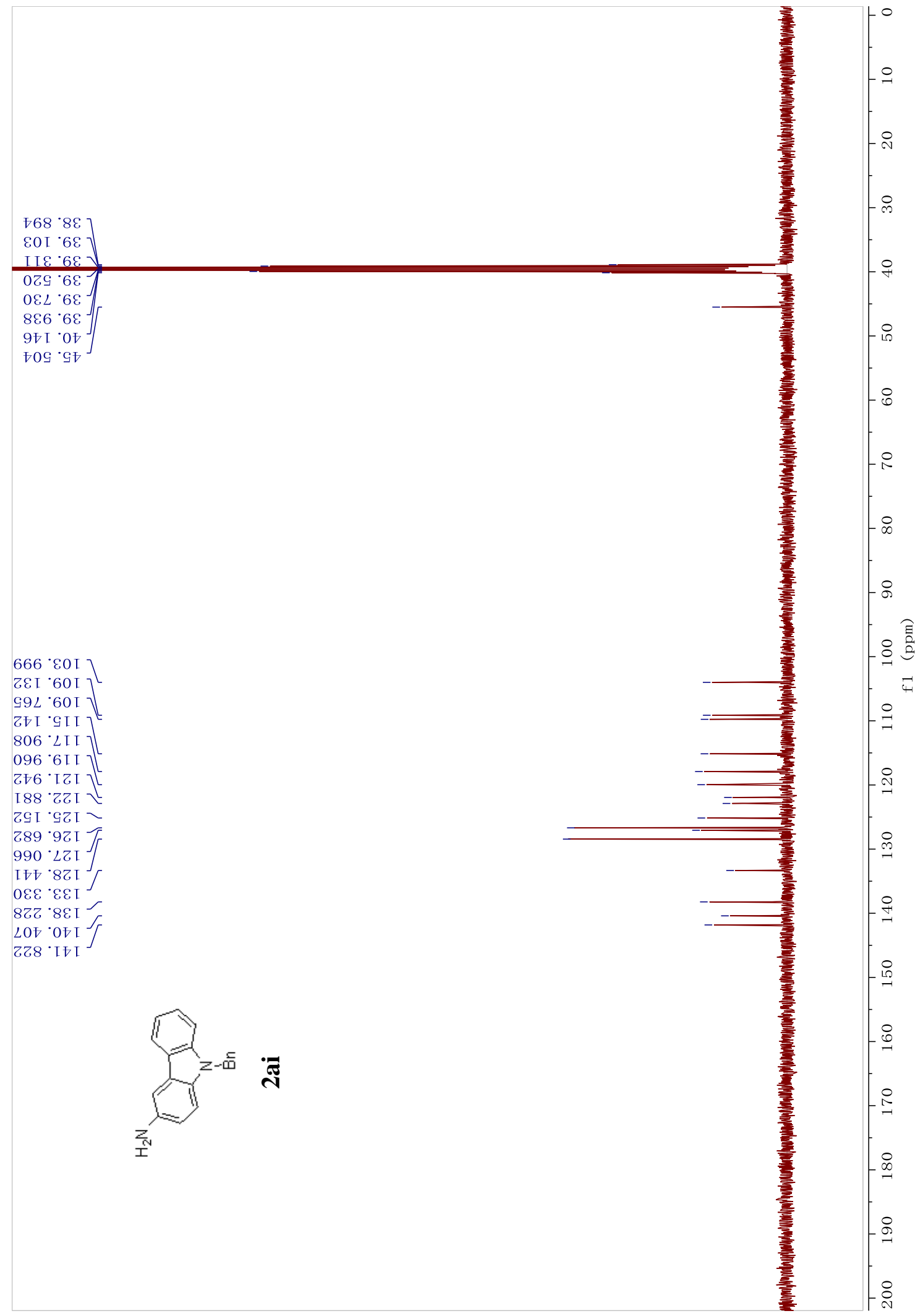




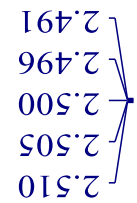
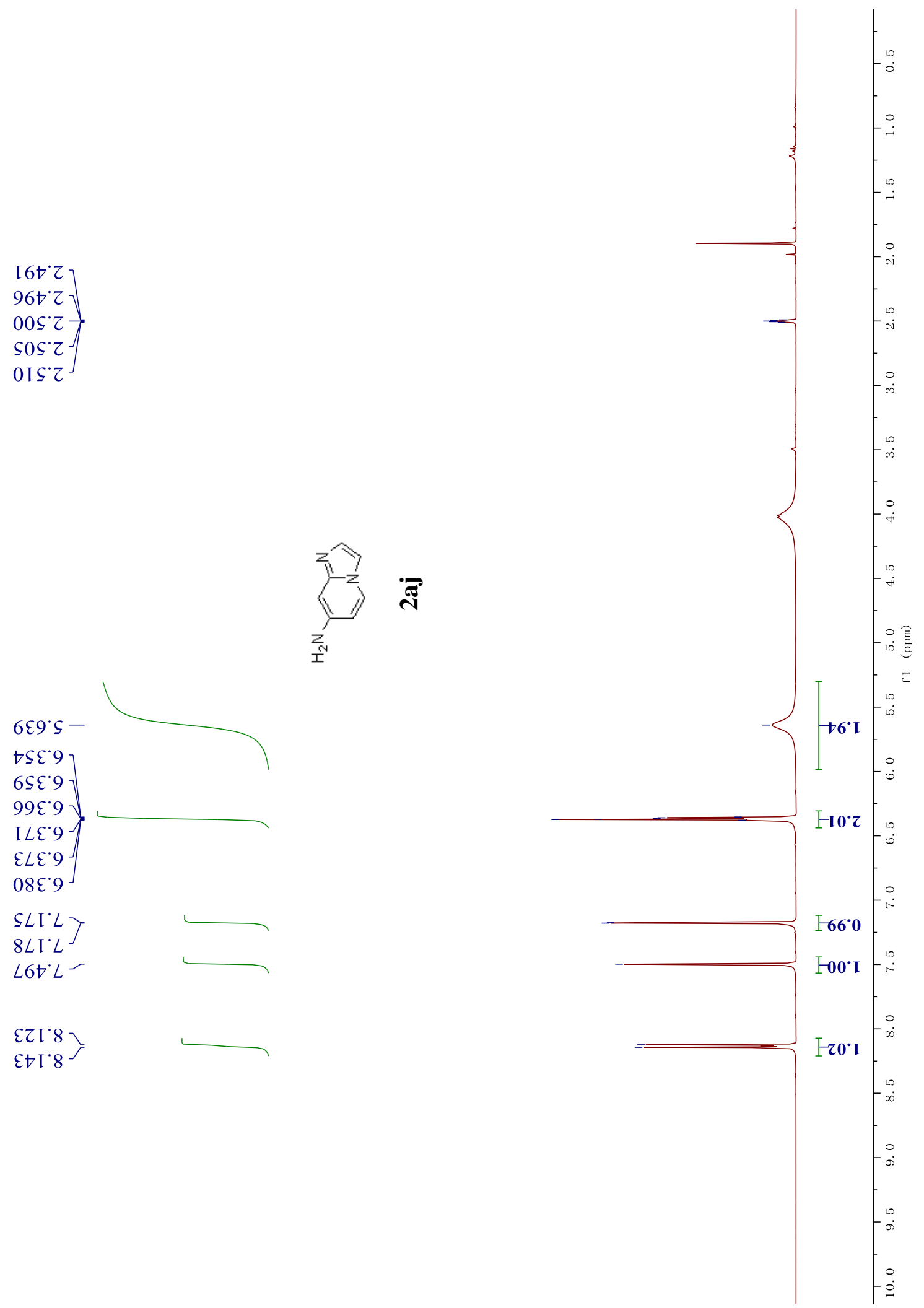


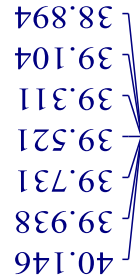

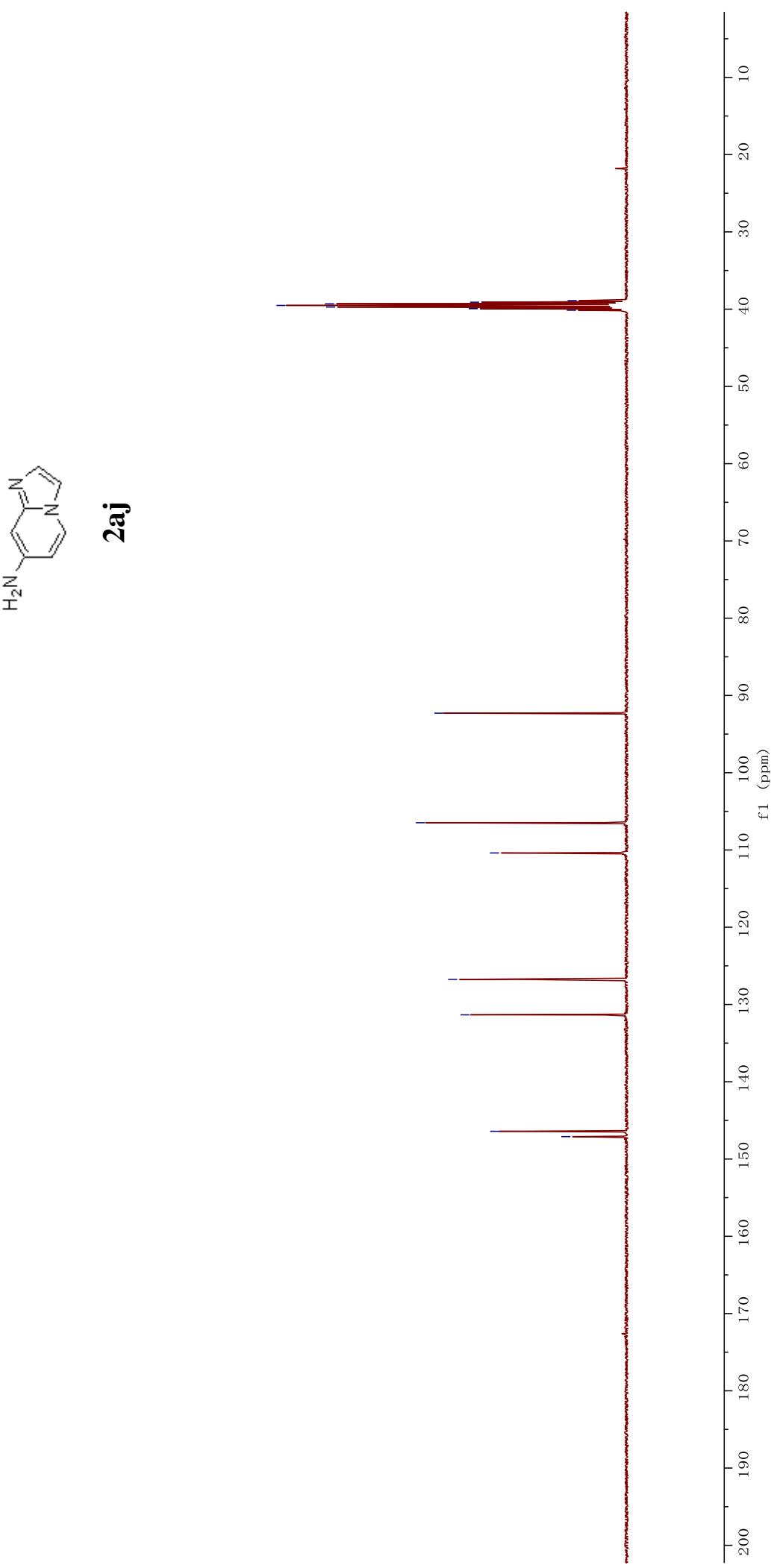

SカL'9ZI -

LEE IEI -

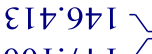

$00 \mathrm{I}^{\circ} \mathrm{Lt} \mathrm{I}^{\mathcal{O}}$

†Lナ'90I -

$68 \varepsilon^{\circ} 0$ I I - 
$\varepsilon L I^{\prime} Z-$

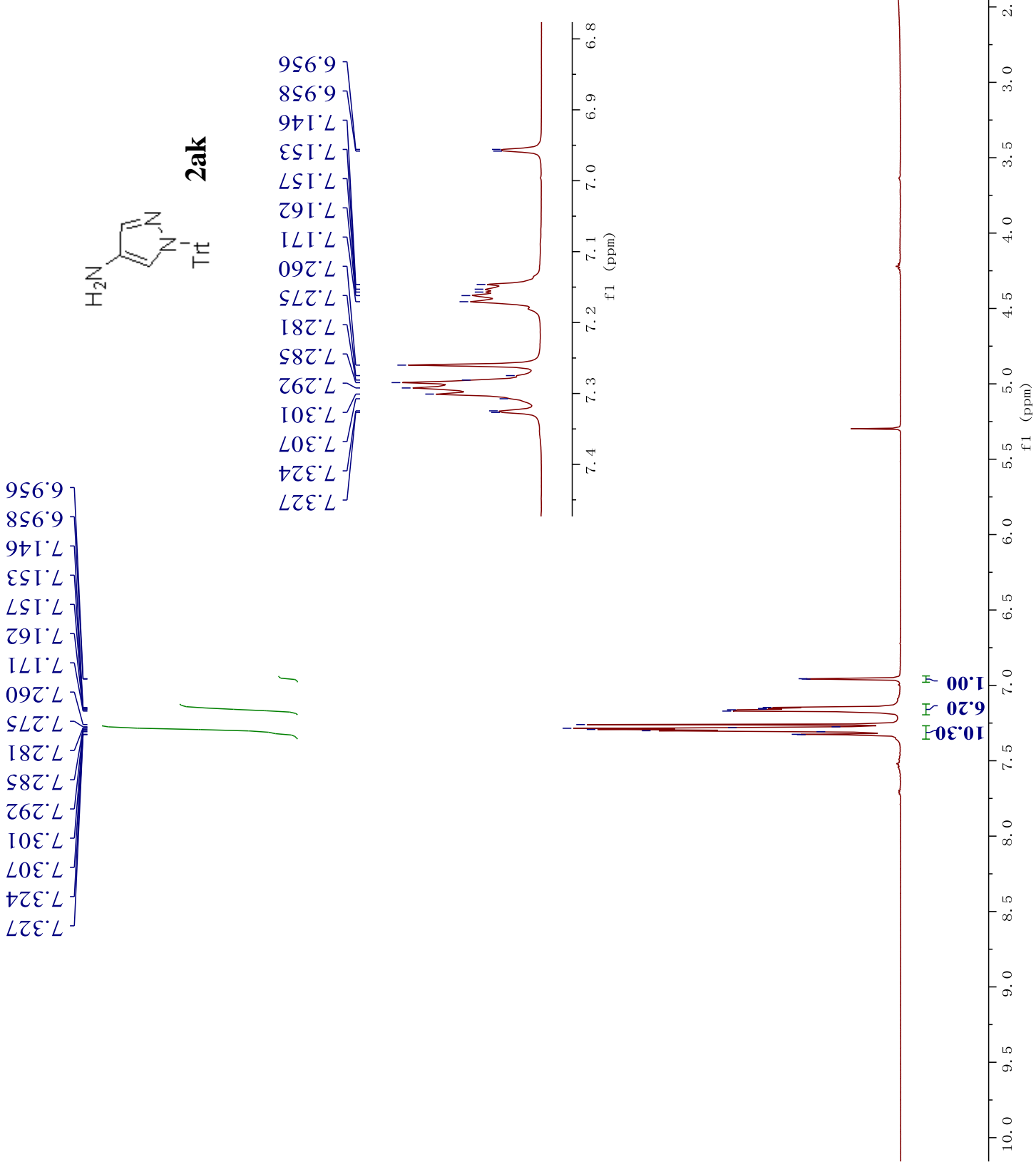



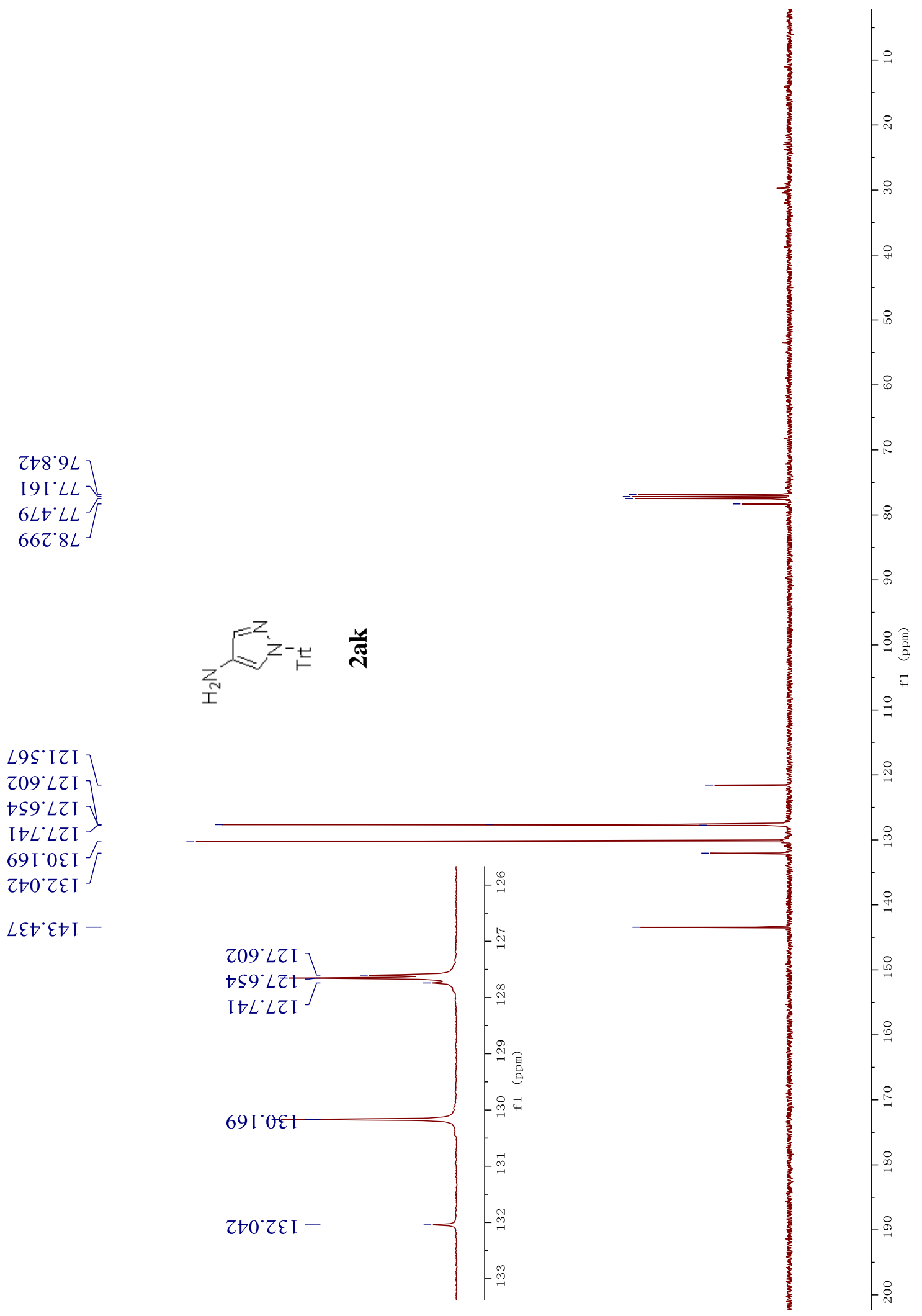
\$99. I -

$\varepsilon 80^{\circ}$ ๑一
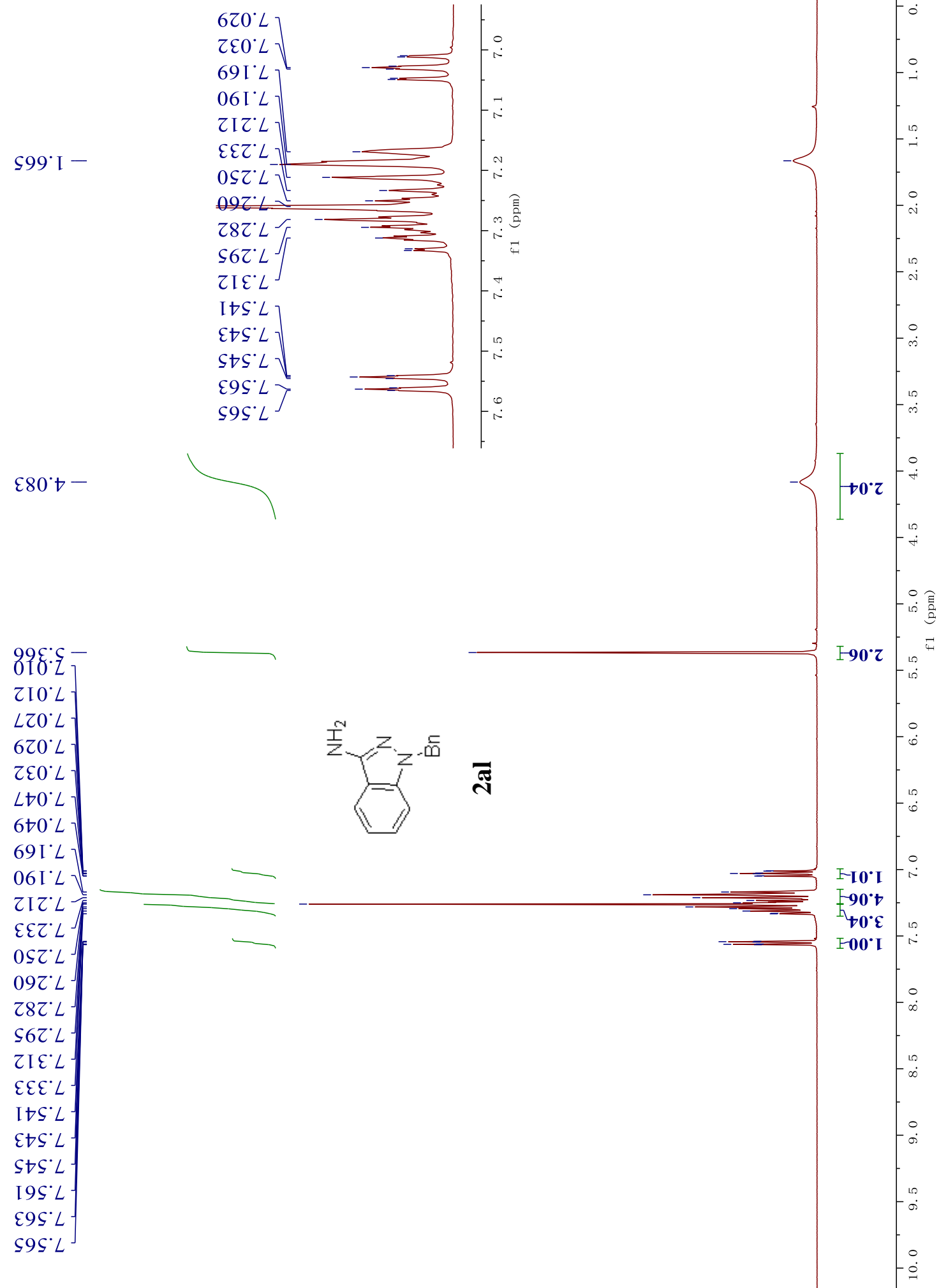
$\left.\begin{array}{l}\varepsilon \sqcup 8^{\circ} 9 L \\ 19 I^{\circ} L L T \\ 6 L \nabla^{\circ} L L\end{array}\right]$

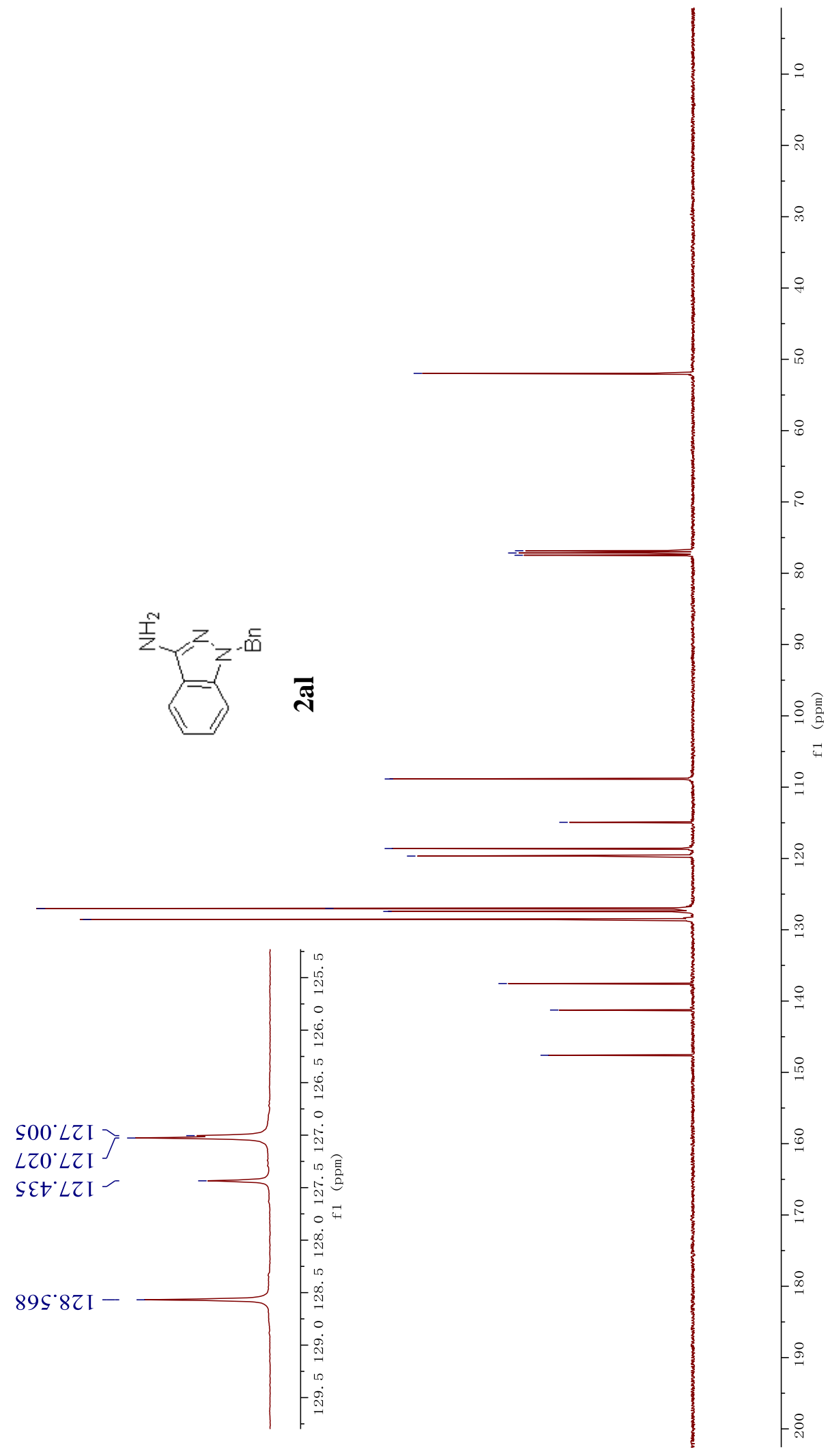




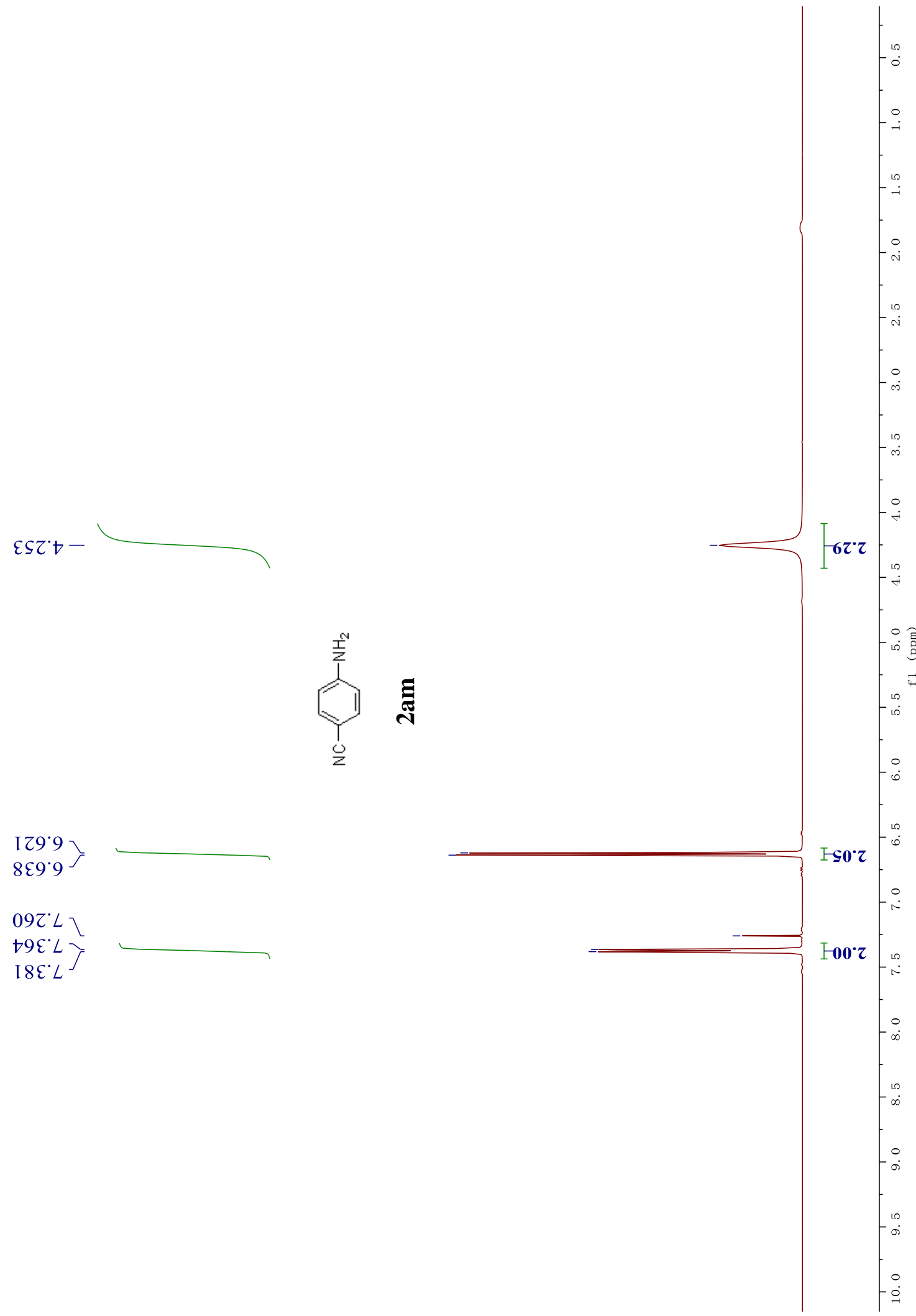


E[०ро [06.9L

E[วpo $s 9]^{\circ} L L \frac{T}{T}$

ย[วро $L I \nabla^{\circ} L L$

8SL'66-

6Eع $0 Z$ I -

S8L'EE I -

$\mathcal{E} L^{\circ} O S I-$
สี

$-2$

$\infty$

ஃ

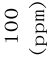

$\overline{5}$

$\stackrel{\circ}{\exists}$

$\stackrel{ }{\mathcal{N}}$

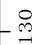

워

$\stackrel{\circ}{\rightarrow}$

$\stackrel{8}{0}$

$\stackrel{ }{ }$

$\stackrel{\infty}{\stackrel{\infty}{-}}$

$\stackrel{\circ}{\circ}$

$-\stackrel{\circ}{\circ}$ 

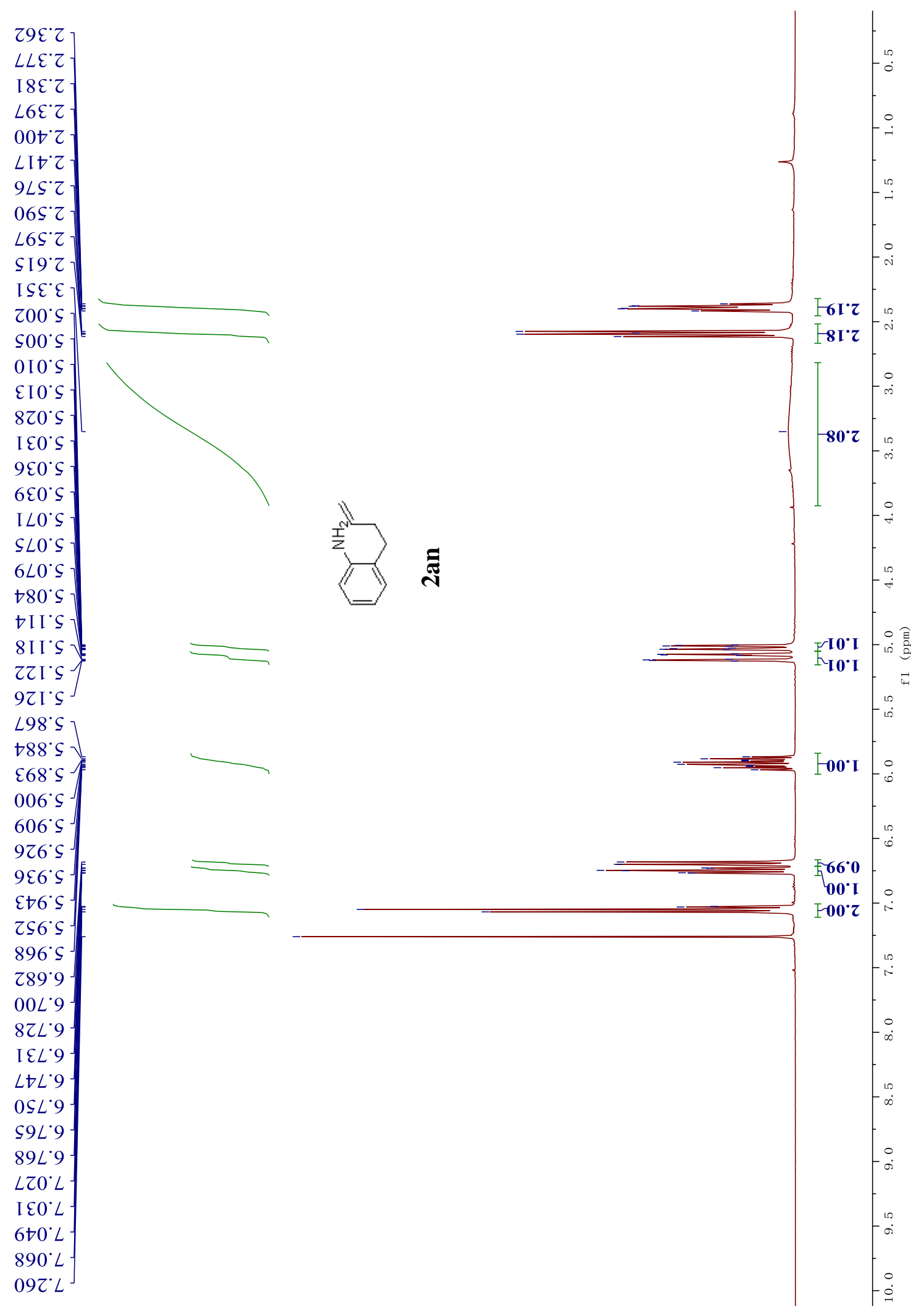
$96 L^{\circ} 0 \varepsilon-$ 乙†6 $ح \varepsilon^{-}$

$\varepsilon \succsim 8^{\circ} 9 L$

$09 I^{\circ} L L \frac{1}{j}$
$8 \angle \nabla^{\circ} L L$

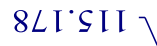
$689^{\circ}$ S I I 8S8.8 I I $\varepsilon \sqcup 0^{\circ} 9$ ZI $\downarrow \varepsilon\left[I^{\circ} L Z I J\right.$ $667^{\circ} 62 \mathrm{I}$

$\varepsilon 乙 て \cdot 8 \varepsilon \mathrm{I}-$

s9I $t \downarrow$ I -

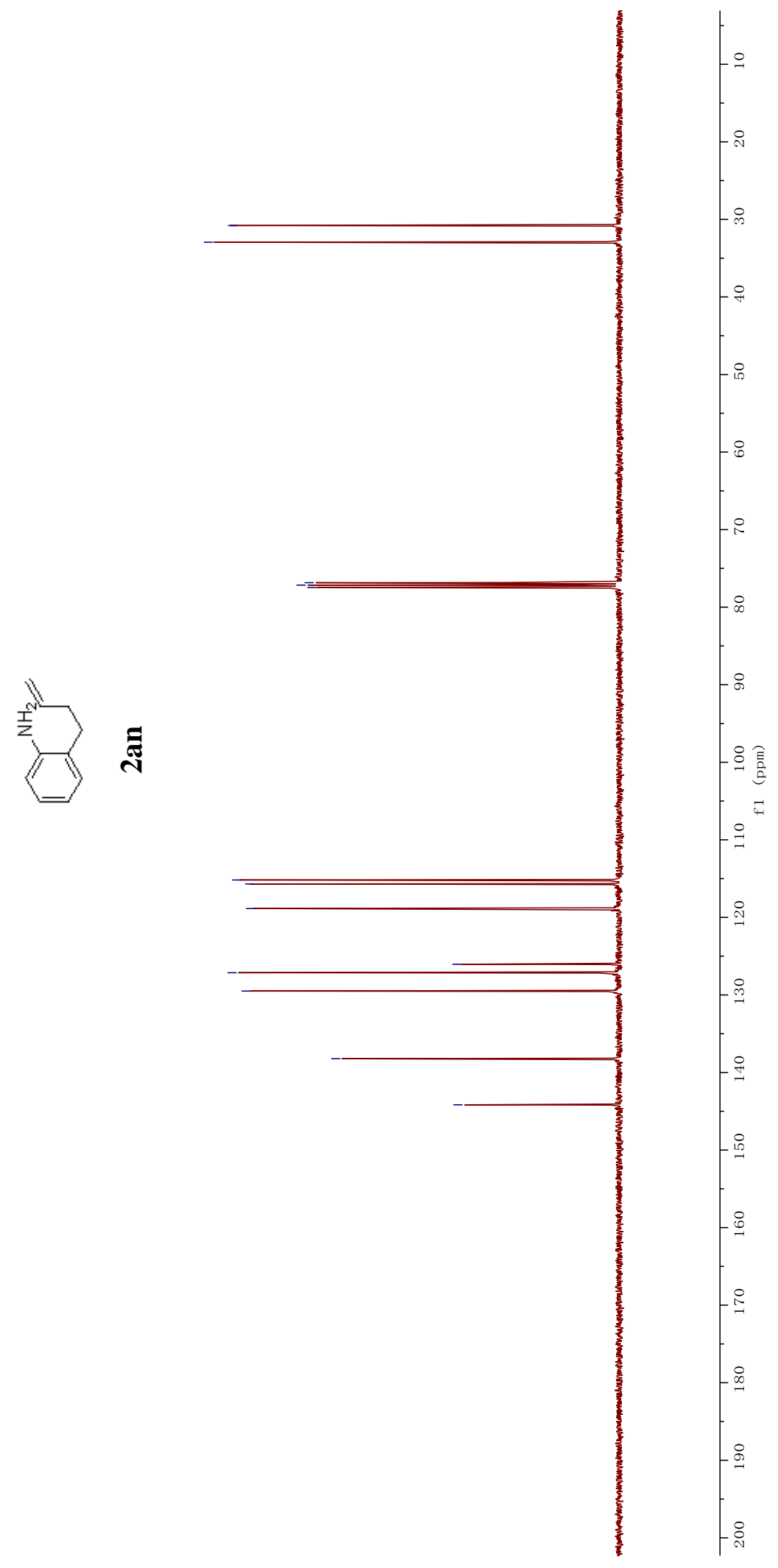

